\title{
Diagnostic challenges during the Dutch $Q$ fever outbreak
}

Citation for published version (APA):

Wegdam-Blans, M. C. A. (2014). Diagnostic challenges during the Dutch Q fever outbreak. [Doctoral Thesis, Maastricht University]. Maastricht University. https://doi.org/10.26481/dis.20140117mw

Document status and date:

Published: 01/01/2014

DOI:

10.26481/dis.20140117mw

Document Version:

Publisher's PDF, also known as Version of record

\section{Please check the document version of this publication:}

- A submitted manuscript is the version of the article upon submission and before peer-review. There can be important differences between the submitted version and the official published version of record.

People interested in the research are advised to contact the author for the final version of the publication, or visit the DOI to the publisher's website.

- The final author version and the galley proof are versions of the publication after peer review.

- The final published version features the final layout of the paper including the volume, issue and page numbers.

Link to publication

\footnotetext{
General rights rights.

- You may freely distribute the URL identifying the publication in the public portal. please follow below link for the End User Agreement:

www.umlib.nl/taverne-license

Take down policy

If you believe that this document breaches copyright please contact us at:

repository@maastrichtuniversity.nl

providing details and we will investigate your claim.
}

Copyright and moral rights for the publications made accessible in the public portal are retained by the authors and/or other copyright owners and it is a condition of accessing publications that users recognise and abide by the legal requirements associated with these

- Users may download and print one copy of any publication from the public portal for the purpose of private study or research.

- You may not further distribute the material or use it for any profit-making activity or commercial gain

If the publication is distributed under the terms of Article $25 \mathrm{fa}$ of the Dutch Copyright Act, indicated by the "Taverne" license above, 
Diagnostic challenges during the Dutch $Q$ fever outbreak 
Sponsoren:

St. Anna Ziekenhuis

Elkerliek ziekenhuis

Catharina Ziekenhuis

Maxima Medisch Centrum

Stichting PAMM

Mediphos Medical Supplies BV

InnatOss Laboratories B.V.

Copyright ( 2013 Marjolijn Wegdam-Blans, Maastricht ISBN 978-90-9028028-8

Layout: Nicole Jacobs-Verhofstad

Cover design: Harry Wegdam

Production: DWMC BV 
"Voor mijn ouders" 



\title{
Diagnostic challenges during the Dutch $Q$ fever outbreak
}

\author{
PROEFSCHRIFT \\ ter verkrijging van de graad van doctor \\ aan de Universiteit Maastricht, \\ op gezag van Rector Magnificus, prof. dr. L.L.G. Soete \\ volgens het besluit van het College van Decanen, \\ in het openbaar te verdedigen \\ op vrijdag 17 januari 2014 om 16.00 uur \\ door \\ Maria Catharina Aleida Wegdam-Blans \\ geboren te Leiderdorp op 23 februari 1970
}




\section{Promotoren}

Prof. dr. J.A.W. Teijink

Prof. dr. M.P.G. Koopmans

\section{Copromotoren}

Dr. H.T. Tjhie

Dr. H.A. Bijlmer

\section{Beoordelingscommissie}

Prof. dr. C.J.P.A. Hoebe (voorzitter)

Prof. dr. P.H.M. Savelkoul

Mw. dr. A. Oude Lashof

Dr. C.M. Verduin 


\section{Contents}

Chapter 1 General introduction and thesis outline

Chapter 2 Evaluation of commonly used serological tests for the detection of Coxiella burnetii antibodies in well-defined acute and follow-up sera

Chapter 3 A dynamic case definition is warranted for adequate 49 notification in an extended epidemic setting: the Dutch $Q$ fever outbreak 2007-2009 as exemplar

Chapter 4 Chronic $Q$ fever: review of the literature and a proposal of new diagnostic criteria

Chapter 5 Chronic $Q$ fever diagnosis: consensus guideline versus expert opinion

Chapter 6 Serology in chronic $Q$ fever is still surrounded by questionmarks

Chapter 7 Vascular complications of $Q$ fever infections

Chapter 8 David procedure during a reoperation for ongoing chronic $Q$ fever infection of an ascending aortic prosthesis

Chapter 9 Targeted screening as a tool for early detection of chronic $Q$ fever patients after a large outbreak

Samenvatting

Dankwoord

Curriculum Vitae 

Chapter 1

General introduction and thesis outline 


\section{Coxiella burnetii and $Q$ fever}

\section{History}

$Q$ fever is a zoonotic disease, caused by Coxiella burnetii (C. burnetii). "Q" stands for "query", referring to a lack of medical knowledge when its clinical syndrome was first described. First reports were described by Derrick in Australia and almost simultaneously by Davis and Cox in the USA in 1937 $[1,2]$. In Queensland, Australia, an outbreak of an unknown infectious agent was observed in abattoir workers. In the search of the causative agent, Derrick inoculated guinea pigs with patient blood. Although the injected animals did develop a febrile disease, Derrick was unsuccessful in demonstrating microorganisms in guinea pigs tissue or cultivating them [2]. A second attempt by Australian colleagues Burnet and Freeman yielded positive smears in spleen tissue [3]. The bacteria were described as rickettsial structures. In the same year, 1937, the afore-mentioned scientists David and Cox discovered an unknown agent while working at Rocky Mountain spotted fever in the village of Nine Mile, Montana [1]. They demonstrated the presence of a different febrile illness when inoculating guinea pigs using infected ticks. As in the earlier attempts, the visualized microorganisms could not be cultured on culture media used at the time. The agent had, according to Davis and Cox, both bacterial and viral features. Eventually, one of Koch's postulates for causality was, unfortunately, met by the director of the national institute of health, Rolla Eugene Dyer, who accidentally became ill due to a laboratoryacquired infection [4]. He displayed symptoms very similar to the recently described " $Q$ fever". This febrile illness was reproduced in guinea pigs inoculated with Dyer's blood, and again rickettsiae structures were identified in spleen samples from infected animals. To honor its discoverers, the bacterium was named after Cox en Burnet.

\section{Microorganism}

C. burnetii is a coccobacillus with a Gram-negative cell wall [5]. It is an obligate intracellular bacterium that shares morphologic characteristics with Rickettsiae and Legionella species [6, 7]. Based on molecular taxonomy, C. burnetii is nowadays considered part of the order Legionellales [6]. Cultivation of these bacteria is difficult: standard culture agars do not suffice; instead cell layers or Yolk sacs of embryonated hens' eggs are required for 
cultivation $[8,9]$. Recently a systematic evaluation of the organism's metabolic requirements has led to development of a complex nutrient cell free medium that supported substantial growth of $C$. burnetii in a $2.5 \%$ oxygen environment.

C. burnetii is characterized by antigenic phase variation, which is mainly based on the composition of lipopolysaccharides (LPS) [10-12]. Phase I is the natural phase found in infected animals. In this phase, the bacterium is highly infectious. Phase II, in contrast, is not so contagious and is obtained in laboratories after several passages in cell cultures [12, 13]. The shift from phase I to phase II occurs as a result of the loss of methylated sugars and deletion of genes associated with LPS synthesis [10,12]. The loss of virulence, which is attended with this deletion, is demonstrated by higher complement sensitivity of phase II antigen in contrast to phase I [12, 14]. Serological diagnosis is based on this transition: with an acute or recent infection, levels of antibodies against the phase-II antigen are elevated, while with chronic infection, antibodies against the phase-I antigen abound [15].

Next to the LPS variation $C$. burnetii also has different morphological variants: Small and Large Cell Variants (SCV and LCV) [16]. The SCV is the form found in the environment and is highly resistant to heat and other environmental stressors and can therefore survive for years outside a host [17]. After infection of cells, intracellular development of $C$. burnetii leads to LCV $[18,19]$. Dependent on the bacteria's living conditions, LCV will either duplicate to form more LCV cells, or the SCV-form will resurface [5, 18-20]. It has been suggested that $C$. burnetii can take a third form, i.e. spores [16]. However, there is no genomic research to date to support this hypothesis, as genes responsible for this formation have not been demonstrated thus far [21].

\section{Clinical manifestations}

Infection with $C$. burnetii in humans mainly results from inhalation of contaminated aerosols and may result in acute and chronic infections [22, 23].

Acute $Q$ fever

Clinical manifestations occur in approximately half of the acute $Q$ fever cases after an incubation period of two to four weeks [22, 23]. Moreover, clinical symptoms may be indiscriminative and vary from a flu-like illness to a more severe syndrome with high fever, severe headaches, gastrointestinal com- 
plaints, pneumonia and hepatitis [11]. It is hypothesized that the severity of the disease is related to the inoculum size [24]. Mortality in acute $Q$ fever is reported around $1 \%[11,25]$.

\section{Chronic $Q$ fever}

In the literature, the percentages of patients progressing to chronic $Q$ fever are estimated at $1-5 \%$ [11, 26-30]. This progress usually occurs insidiously, months or even years after primary infection. Such patients often present with non-specific symptoms such as low-grade fever, night sweats and weight loss [26, 27, 29-33]. Before the Dutch outbreak (2007), endocarditis was described as the predominant manifestation of chronic $Q$ fever, followed by vascular wall infection, chronic infection in pregnancy, osteomyelitis and chronic hepatitis $[30,32]$. Both symptomatic acute $Q$ fever cases, as well as patients who displayed no or asymptomatic symptoms while having contracted acute $Q$ fever, are at risk of developing chronic infection. Known risk factors for developing chronic infection are valvular heart disease, including heart valve prostheses, vascular prostheses and aneurysmatic diseases of the aorta [26, 27, 30-32]. Unlike acute $Q$ fever, chronic $Q$ fever is a potentially lethal disease. Morbidity and mortality rates are related to the time of diagnosis and the start of combination antibiotic therapy and surgical intervention [29].

\section{Vascular infection}

In the literature, it is stated that vascular-related infections are the second most frequent manifestation of chronic $Q$ fever after endocarditis [26, 32]. Vascular infections probably account for $9-13 \%$ of the chronic $Q$ fever cases [32]. However, due to the unfamiliarity of this phenomenon, this percentage may very well be an underestimate. Both infectious aneurysms and vascular graft infections occur [26, 32, 34-38], and mortality is high. Infections of vascular prostheses often have a slow progression. In due course, abscesses and/or aortoenteric or aortobronchial fistulas may arise. Clinical symptoms described in patients with vascular-related $Q$ fever infections are usually fever, weight loss, abdominal pain and fatigue [26, 32, 34-38]. Various articles in the literature give a description of a total of approximately 50 individual cases [26, 32, 34-38]. This number will increase significantly as a result of the recent cases in the Netherlands. 


\section{Treatment}

In principal, acute $Q$ fever is a self-limiting disease; antibiotics can reduce the duration of symptoms if prescribed within the first days of illness. Because $C$. burnetii is an intracellular living microorganism, the range of usable antibiotics is limited. The efficacy of antibiotics in acute $Q$ fever has mainly been tested using embryonated-egg and cell culture models. For susceptibility of $C$. burnetii to antibiotics the same models have been applied. In these experiments good bacteriostatic or bacteriocidal activity against $C$. burnetii was demonstrated for doxycycline, co-trimoxazole, rifampicin, a new generation of quinolones (levofloxacin and moxifloxacin) and macrolides (especially clarithromycin) [39-43]. Human studies are scarce and mainly retrospective. In one randomized controlled trial, doxycycline was proven to have a better efficacy in terms of a shorter duration of symptoms as compared to erythromycin [44]. All studies combined, the first choice of treatment is doxycycline, followed by the newer quinolones or macrolides. In the Netherlands, doxycycline 200mg per day for 14 days is recommended [45].

Treatment of chronic $Q$ fever is also based on infected cell models combined with clinical experience. Mimicking chronic infection, cell lines were infected for a prolonged period (400 days or more). On the basis of these models, it was demonstrated that mono antibiotic treatment habitually failed, leading to relapse [41]. It was discovered that combining doxycycline with a lysosomotropic alkalinizing agent would lead to a significant reduction of infected cells. An augmented intracellular $\mathrm{pH}$-level increased the antibiotic activity of doxycycline $[39,41]$. Based on these experiments, guidelines were drawn up recommending treating chronic $Q$ fever patients with doxycycline and hydroxychloroquine. Dose regimen of both agents should be individually determined based on concentrations in blood [45].

\section{Dutch outbreak}

From 2007 to 2009, the Netherlands experienced the world's largest outbreak of $Q$ fever, with more than 4,000 reported cases of acute $Q$ fever [4652].

C. burnetii had been circulating at low levels in the Netherlands before 2007 . In a population-based seroprevalence study carried out in 2006, a seroprevalence of approximately $2 \%$ was demonstrated [53]. A higher seroprevalence was associated with increasing age, male gender and occupational 
contact with animals. No geographical differences were observed. This study was performed using serological techniques that are customarily applied in the current diagnostic work-up of $Q$ fever. Older seroprevalence studies from the Netherlands showed higher percentages, especially in people with (occupational) risk for $Q$ fever [54-56]. However, these studies were performed using an in-house indirect fluorescent antibody test (IFAT) and were considered positive when IgM phase II was $\geq 1: 16$. A considerable amount of the demonstrated seroprevalence was therefore probably based on falsepositive reactions and misclassification - an assumption that is reinforced by that fact that $Q$ fever notification was 20 cases a year at the time. In conclusion, $Q$ fever had been circulating at low levels in the Netherlands before the start of the outbreaks in 2007 to 2009.

The Dutch outbreak started during spring 2007 in the village of Herpen. A general practitioner reported an excess of patients with atypical pneumonia in his practice. Initially, these patients were diagnosed with Mycoplasma pneumoniae based on positive serology. Later it was recognized that false positivity based on cross-reactivity was the cause of these diagnoses of Mycoplasma pneumoniae infection, and that the actual, underlying disease was indeed $Q$ fever. Afterwards the epidemic spread across the (mainly rural) provinces of Noord-Brabant, Gelderland and Limburg [49-51]. The outbreak continued for three years and annually more patients were reported with a maximum of 2,313 patients in 2009 (figure 1). An annual seasonal peak was clearly observed; most patients were identified during spring, during the lambing season. Dairy goats were already identified as the source of the human epidemic in 2007; the Dutch Animal Health Service (Gezondheidsdienst voor Dieren (GD)) confirmed a considerable number of $Q$ fever induced abortions at goat farms in the aforementioned areas. These abortions were accompanied by high loads of the bacterium through aborted lambs, placentas and amniotic fluids [11, 23]. The environment was highly contaminated with aerosols containing $C$. burnetii, which can spread for kilometres from its initial source [11, 23]. Although the epidemic's presumable source was already traced in 2007 , it took three years to convince the national authorities. Only by the end of 2009, drastic veterinary measures were taken, such as the culling of goats from $Q$ fever-positive goat farms and the prohibition of manure transports, resulting in a steep decline in the epidemic in 2010. Before the human $Q$ fever outbreak there were already reports about positive farms and concerns about the potential harm for humans. 


\section{Diagnostic tools at the start of the outbreak}

Although $C$. burnetii can be cultured, this is restricted to research and reference centers only: it requires a level 3-biosafety laboratory. Moreover, sophisticated culture techniques are required, such as cell layers, which are also used for culturing viruses or Yolk sacs $[8,9]$. It takes weeks before inoculated material becomes positive. Therefore, in routine practice, the diagnosis of $Q$ fever is based on DNA detection with polymerase chain reaction (PCR) and antibody determination.

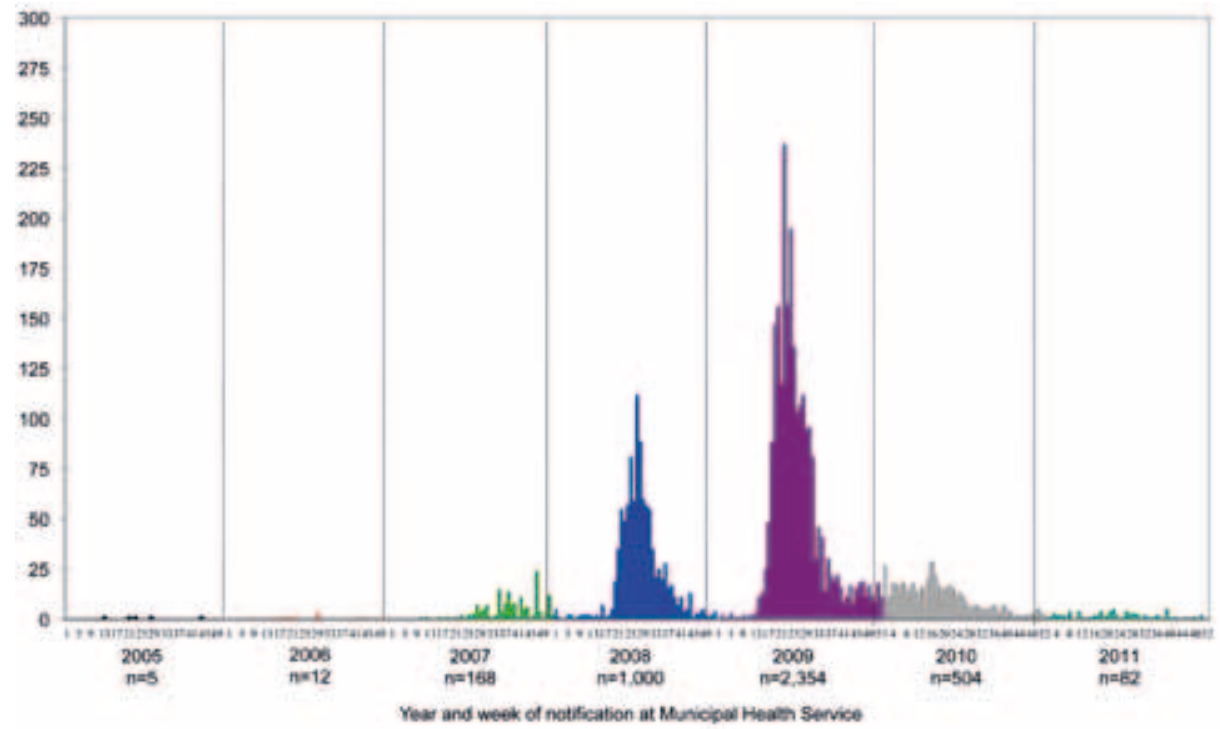

Figure 1. Number of notified acute $Q$ fever patients in the Netherlands (left axis) by week of notification (right axis), from $1^{\text {st }}$ of January 2007 - December 2011. Adapted with permission from L. Verhoef from the National Institute for Public Health and the Environment (RIVM).

\section{Diagnosis of acute $Q$ fever}

PCR

Flu-like symptoms occur after an incubation period of 2-3 weeks, with a possible extension to 6 weeks. On average, DNA is detectable in peripheral blood directly from the start until two to three weeks after the first occurrence of the symptoms [57-59]. PCR sensitivity in the first days after start of symptoms is reaching $100 \%$, but rapidly declines to undetectable levels [57-59]. After concerted validation, most laboratories in the Netherlands implemented the same $Q$ fever-PCR from 2009, based on a real-time PCR targeting 
IS1111 gene [59, 60]. The PCR has been validated for serum as well as whole blood, in acute $Q$ fever patients. It is thus far unknown whether this method is also applicable for other body materials, such as respiratory material. There are studies in which a multiplex PCR targeting different respiratory pathogens, among $C$. burnetii, is validated in particular patients groups [61]. However, to date, PCR from respiratory material was not done routinely in the Dutch laboratories.

\section{Serology}

As mentioned above, detection of $C$. burnetii DNA in serum with PCR is highly time-dependent, and sensitivity decreases to low levels over the course of two to three weeks after onset of acute $Q$ fever symptoms. $Q$ fever diagnostic tests are not always required initially; therefore serological tests will always be necessary in acute $Q$ fever diagnostics. Serological diagnosis is based on crude bacterial lysates representing the two antigenic phases, phase I and phase II [15]. With an acute or recent infection, levels of antibodies against the phase-II antigen are elevated, while with chronic infection, antibodies against the phase-I antigen predominate. In both phases, $\lg \mathrm{M}$ as well as $\lg \mathrm{G}$ antibodies can be measured. After a serological 'window phase' (i.e. the phase in which antibodies are not yet found), IgM emerges first [15]. It is traceable circa 2-3 weeks after the first occurrence of the disease's symptoms, around the moment that the PCR assays become negative (figure 2). There are various serological methods to detect these antibodies that are used in Dutch laboratories [62-69].

\section{Acute Q fever}

Serum tests

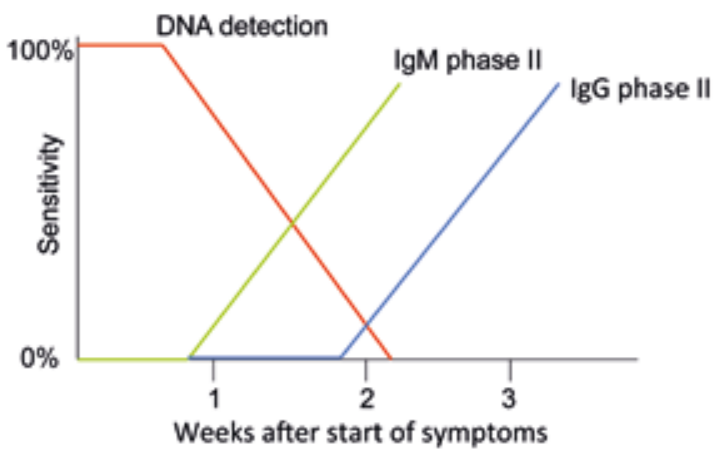

Figure 2. Detection of DNA with PCR and antibody response related to the first day of onset of illness. 
Complement Fixation Test (CFT) detects IgM as well as IgG antibodies together without discrimination [70]. Phase I and phase II antibodies are detected separately using two different antigen compositions. CFT is based on the depletion of complement proteins as a consequence of the formation of antigen-antibody complexes. The test is conducted in serum dilutions [7072]. A fourfold titer increase or a seroconversion is considered to be diagnostic for recent infection. The CFT reportedly has both a high specificity as well as sensitivity, although it seems that CFT-antibodies appear slightly later in the disease's development than antibodies detected with other methods [68, 73]. CFT is a labor-intensive test, for which proper analytical expertise is required. Furthermore, the method, similar to immune-fluorescence assay, is sensitive to inter- and intra-observer variation.

As has been described above, $C$. burnetii displays antigenic phase variation. This can be demonstrated using IFAT, whereby four determinants (IgG and IgM plotted against Phase I and Phase II) are being analyzed. In the literature, IFAT is considered the reference method for serological diagnosis as it can distinguish between acute, past and chronic infection based on the levels of these four antibodies [74-76]. Like CFT, the IFAT method is a more laborious technique and interpretation can be subjective and is therefore sensitive for inter- and intra-observer variation. IFAT is also conducted in serum dilutions, and, similar to CFT, either a fourfold titer increase or a seroconversion can serve as proof of a recent infection. Seroconversion can typically be expected in 2 weeks' time, as can a fourfold titer increase with most patients [74, 77]. However, practical observations have shown variations in the antibody profile in different patients; some patients display slow or low levels of antibody generation, which implies that a 2-week interval may not be sufficient.

Enzyme-Linked Immunosorbent Assays (ELISA) to measure presence of antibodies to IgG phase I, IgG phase II, IgA phase I and IgM phase II are commercially available. For acute $Q$ fever diagnosis, only ELISA-IgM phaseII testing is routinely applied throughout the various algorithms that are used nationally (figure 3 ). The ELISA-IgM phase-II test readouts can be programmed on an ELISA-reader; they are easiest to conduct of the available three types of serological tests, and are therefore suitable as a screening assay. With an assessed operational sensitivity of 99\%, ELISA-IgM phase-II test results after the serological window phase are very good [65]. 


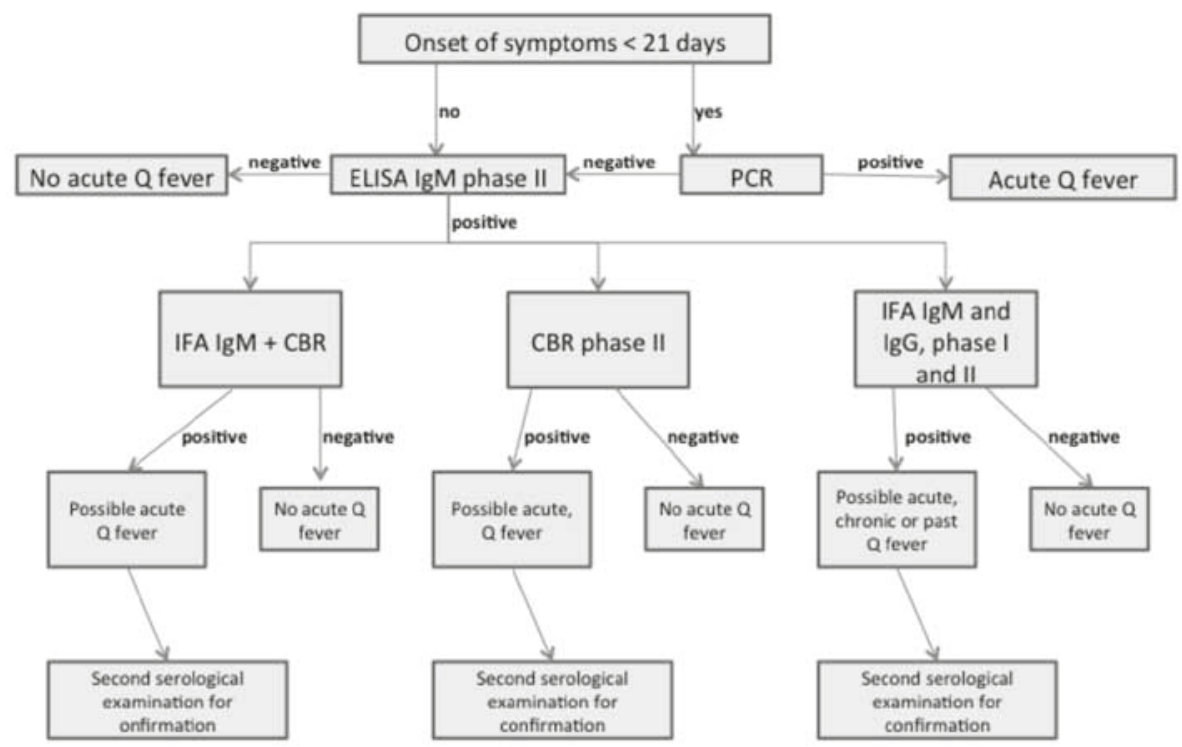

Figure 3. Diagnostic algorithms of acute $Q$ fever. From Wegdam-Blans et al. [98].

\section{Limitations of serology}

With all three methods, cross-reactivity due to presence of antibodies to other pathogens may be present. False positive (IgG) phase II reactions have been described in cases of acute Legionella, Bartonella and Chlamydophila [78-84]. Laboratory diagnosis of acute $Q$ fever, on a basis of a solitary sample, is often not possible because of the lack of clinical data, including the duration of symptoms at the time of sampling. Furthermore, the background prevalence dictates the specificity of a diagnosis based on a solitary serum with a positive IgM test result (ELISA and/or IFAT).

\section{Notification}

In the Netherlands, acute $Q$ fever has been notifiable since 1976. In case an infection is detected, the head of the medical microbiology laboratory and the diagnosing physician are obliged to notify the local public health authorities (the Municipal Health Services, MHS) [85]. Subsequently, the MHS must report and provide data to the National Case Register (NCR) of notifiable diseases. In accordance with the Public Health Act, the test results should remain anonymous [85]. Notification of acute $Q$ fever is mandatory in cases of: 
- Solitary positive IgM Phase-II outcomes (ELISA and/or IFA), in cases where medical signs also point towards $Q$ fever (possible acute $Q$ fever) [45];

- Positive PCR-results, but only in cases of acute illness (proven acute Q fever);

- Serologically confirmed acute $Q$ fever infections on the basis of a fourfold titer increase in CFT- or IFA-IgG Phase-II (proven acute $Q$ fever).

\section{Diagnosis of chronic $Q$ fever}

If left untreated, chronic $Q$ fever leads to considerable morbidity and mortality, which can rate up to $60 \%$ [29]. Long-term antibiotic treatment and sometimes aggressive surgery are required in patients with established chronic $Q$ fever [26, 29]. Therefore, it is of vital importance that suspected chronic $Q$ fever patients receive adequate diagnoses. The diagnosis of chronic $Q$ fever presents challenges: culturing $C$. burnetii is difficult and time-consuming; it requires a level 3-biosafety laboratory and the methodology lacks sensitivity [9].

PCR

The validation of the PCR method used is limited to acute $Q$ fever. To date, its sensitivity and specificity for chronic $Q$ fever are undetermined: the medical science does not know if, in what form, and in what compartment DNA of C. burnetii can be detected in the different chronic $Q$ fever entities. Detection of the bacterium by DNA amplification or culturing is a solid marker for the disease, and therefore superior to antibody detection.

\section{Serology}

Due to the limitations of PCR and culturing pointed out above, the laboratory diagnosis of chronic $Q$ fever mainly relies on serology. Persisting high levels of antibodies to phase I and phase II antigens are thought to be caused by continuous antigenic stimulation and are considered indicative for chronic $Q$ fever [86, 87]. High phase I IgG antibody titers are a strong indication of a chronic infection $[11,67,87,88]$. In theory, all serological techniques can be used to establish chronic $Q$ fever, however, IFAT is used most frequently. Especially after the Duke Criteria were changed in 2000 by including a IFAT IgG phase I antibody titer $\geq 1: 800$ as a major criteria, IFAT became the golden standard for chronic $Q$ fever diagnostics [89]. In earlier studies, other 
serology assays were compared in different $Q$ fever patients groups [63, 9094]. Enzyme-Linked Immuno Sorbent Assay (ELISA), CFT as well as IFAT were applied, all using in-house assays on the basis of antigens from different laboratory strains (Herzbergstrain and Nine Mile strain). The overall interpretation of the results of these studies was that marked differences in IgG phase I titers between acute and chronic $Q$ fever patients were observed using all three techniques. However, due to the small number of samples, these differences have never been estimated reliably. Also, comparison of titers across studies was hindered by interlaboratory variation and the difference in dilution series used for CFT tests and IFAT tests (fourfold or fivefold). In early publications, it was suggested that IgA antibodies might play a role in diagnosing chronic infection, i.e. most notable has been the proposal to use IgA as a biological prognostic marker in patients treated for chronic $Q$ fever $[93,95,96]$. However, after 1992 there is no further mention of this suggested usage in the literature.

In more recent studies IgG phase I profiles have been investigated using IFAT. As has been mentioned earlier, a cut-off of IgG phase I $\geq 1: 800$ was proposed for the serological diagnosis of chronic $Q$ fever [89]. This cut-off was based on a retrospective serological study of 53 patients with chronic infections [74]. Later, this cut-off was confirmed by Rolain et al. in 2003: they found no false positive results when $\geq 1: 800$ was used [97].

\section{Thesis outline}

The aims of this thesis are:

1. to do an in-depth analysis of laboratory diagnostics for acute and chronic $Q$ fever in order to improve the applicability of the different tests during daily practice of a routine medical microbiological laboratory. Studies were embedded in a national microbiology consensus group that was formed during the $Q$ fever outbreak in the Netherlands to review existing knowledge as well as new insights gained during the outbreak investigation, and to develop harmonized guidelines for diagnosis and treatment, both for patient care and in support of disease control activities.

2. to gain knowledge of the clinical aspects of chronic $Q$ fever and to improve the diagnostic work-up of chronic $Q$ fever by implementing this knowledge. 
The first chapters deal with the two main questions that arose after the consensus of acute $Q$ fever was established. In chapter 2, the comparison of different serological assays in diagnosing acute $Q$ fever is described using a large number of acute and sequential convalescent serum samples from very well-defined acute $Q$ fever patient groups. In addition the long-lasting serological response is compared between the different tests. Confronted with the largest $Q$ fever epidemic worldwide, the MHS decided to receive rather 'too many than too few' notifications. This entails that possible infections must also be reported to the MHS. Chapter 3 describes the usefulness of this notification rule in the years during and after the epidemic (2009 and 2010-2011 respectively), and how increasing background seroprevalence affected the numbers and the status of the notifications.

After 2009, the acute Q fever epidemic in the Netherlands subsided. Nevertheless, chronic $Q$ fever has been established in a rising number of patients since. Given the uncertainties in diagnostic algorithms for chronic $Q$ fever, the impact of adequate diagnosis on the prognosis and treatment, and the increasing number of patients, it became crucial to perform a systematic review. The results of this review together with a new proposal for the diagnosis of chronic $Q$ fever are described in chapter 4 . Controversy about our proposal merged soon after publication. In chapter $\mathbf{5}$, we applied both the Dutch and a French guideline to all chronic $Q$ fever patients included in the Dutch National Chronic $Q$ fever Database. The diagnosis of chronic $Q$ fever provides us with a challenge. The indication for antibiotic treatment is now supported by the Dutch criteria for chronic $Q$ fever (chapter 4). The relevance of other serological techniques in establishing the diagnosis and their use as a prognostic factor are, as yet, unknown. Therefore, a serological comparison was done, using acute and follow-up sera from chronic $Q$ fever patients, part of whom had received antibiotic treatment and part of whom had not (yet) received such treatment. This is described in full in chapter 6 . The majority of chronic $Q$ fever patients in our region had vascular wall infections. We wanted to increase our knowledge of the manifestations of the disease and the possible complications in these patients. Therefore we conducted a review of the literature to identify the major symptoms of $Q$ fever vascular infection, the most accepted and feasible treatments and their respective outcomes. The results of this review, conjoined with an in-depth analysis of four cases in our institute, are described in chapter 7. Chronic $Q$ fever patients are heterogenic and the therapeutic approach is often tailored 
to the individual patient. In chapter $\mathbf{8}$, we describe a rare case of an ongoing C. burnetii infection of an ascending aorta prosthesis. This case report highlights the challenges regarding the treatment of patients with chronic vascular C. burnetii infections. Eventually, the infection could only be solved after removing the infected prosthesis.

In the aftermath of the Dutch $Q$ fever outbreak, an increasing number of patients are being diagnosed with chronic $Q$ fever. Most of these patients are unaware of being infected with $C$. burnetii. To find patients in an earlier, asymptomatic stage, a targeted screening strategy for patients with risk factors for chronic $Q$ fever was started. The results of this screening are described in chapter 9.

A summary and general discussion of the findings of this thesis is given in chapter 10 , followed by a Dutch summary. 


\section{References}

1. Davis GE, Cox HR. A filter-passing infectious agent isolated from ticks. I. Isolation from Dermacentor andersonii, reactions with animals, and filtration experiments. Public Health Rep 1938;53:2259-76.

2. Derrick EH. "Q" fever, a new fever entity:clinical features, diagnosis and laboratory investigation. Med J Aust 1937;2:281-99.

3. Burnet FM, Freeman M. Experimental studies on the virus of "Q" fever. Med $J$ Aust 1937;2:299-305.

4. Dyer RE. A filter-passing infectious agent isolated from ticks. IV. Human infection. Public Health Rep 1938;53:2277-82.

5. Baca OG, Paretsky D. Q fever and Coxiella burnetii: a model for host-parasite interactions. Microbiol Rev 1983 Jun;47(2):127-49.

6. Stein A, Saunders NA, Taylor AG, Raoult D. Phylogenic homogeneity of Coxiella burnetii strains as determinated by $16 \mathrm{~S}$ ribosomal RNA sequencing. FEMS Microbiol Lett 1993 Nov 1;113(3):339-44.

7. Weisburg WG, Dobson ME, Samuel JE, Dasch GA, Mallavia LP, Baca O, et al. Phylogenetic diversity of the Rickettsiae. J Bacteriol 1989 Aug;171(8):4202-6.

8. Gil-Grande R, Aguado JM, Pastor C, Garcia-Bravo M, Gomez-Pellico C, Soriano F, et al. Conventional viral cultures and shell vial assay for diagnosis of apparently culture-negative Coxiella burnetii endocarditis. Eur J Clin Microbiol Infect Dis 1995 Jan;14(1):64-7.

9. Musso D, Raoult D. Coxiella burnetii blood cultures from acute and chronic Q-fever patients. J Clin Microbiol 1995 Dec;33(12):3129-32.

10. Hackstadt T. Antigenic variation in the phase I lipopolysaccharide of Coxiella burnetii isolates. Infect Immun 1986 Apr;52(1):337-40.

11. Maurin M, Raoult D. Q fever. Clin Microbiol Rev 1999 Oct;12(4):518-53.

12. Vishwanath $\mathrm{S}$, Hackstadt $\mathrm{T}$. Lipopolysaccharide phase variation determines the complement-mediated serum susceptibility of Coxiella burnetii. Infect Immun 1988 Jan;56(1):40-4.

13. Moos A, Hackstadt T. Comparative virulence of intra- and interstrain lipopolysaccharide variants of Coxiella burnetii in the guinea pig model. Infect Immun 1987 May;55(5):114450.

14. Hackstadt $T$. The role of lipopolysaccharides in the virulence of Coxiella burnetii. Ann $N$ Y Acad Sci 1990;590:27-32.

15. Dupuis G, Peter O, Peacock M, Burgdorfer W, Haller E. Immunoglobulin responses in acute $Q$ fever. J Clin Microbiol 1985 Oct;22(4):484-7.

16. McCaul TF, Williams JC. Developmental cycle of Coxiella burnetii: structure and morphogenesis of vegetative and sporogenic differentiations. J Bacteriol 1981 Sep;147(3):106376.

17. Waag DM. Coxiella burnetii: host and bacterial responses to infection. Vaccine 2007 Oct 16;25(42):7288-95.

18. Baca OG, Klassen DA, Aragon AS. Entry of Coxiella burnetii into host cells. Acta Virol 1993 Apr;37(2-3):143-55.

19. Coleman SA, Fischer ER, Howe D, Mead DJ, Heinzen RA. Temporal analysis of Coxiella burnetii morphological differentiation. J Bacteriol 2004 Nov;186(21):7344-52.

20. Baca OG, Li YP, Kumar H. Survival of the $Q$ fever agent Coxiella burnetii in the phagolysosome. Trends Microbiol 1994 Dec;2(12):476-80. 
21. Seshadri R, Paulsen IT, Eisen JA, Read TD, Nelson KE, Nelson WC, et al. Complete genome sequence of the Q-fever pathogen Coxiella burnetii. Proc Natl Acad Sci U S A 2003 Apr 29;100(9):5455-60.

22. Marrie TJ, Stein $A$, Janigan $D$, Raoult $D$. Route of infection determines the clinical manife stations of acute Q fever. J Infect Dis 1996 Feb;173(2):484-7.

23. Parker NR, Barralet JH, Bell AM. Q fever. Lancet 2006 Feb 25;367(9511):679-88.

24. Angelakis E, Raoult D. Q Fever. Vet Microbiol 2010 Jan 27;140(3-4):297-309.

25. Kampschreur LM, Wegdam-Blans MC, Thijsen SF, Groot CA, Schneeberger PM, Hollander AA, et al. Acute $Q$ fever related in-hospital mortality in the Netherlands. Neth J Med 2010 Dec;68(12):408-13.

26. Botelho-Nevers E, Fournier PE, Richet H, Fenollar F, Lepidi H, Foucault C, et al. Coxiella burnetii infection of aortic aneurysms or vascular grafts: report of 30 new cases and evaluation of outcome. Eur J Clin Microbiol Infect Dis 2007 Sep;26(9):635-40.

27. Fenollar F, Fournier PE, Carrieri MP, Habib G, Messana T, Raoult D. Risks factors and prevention of $Q$ fever endocarditis. Clin Infect Dis 2001 Aug 1;33(3):312-6.

28. Healy B, van WH, Raoult D, Graves S, Pitman J, Lloyd G, et al. Chronic Q fever: different serological results in three countries--results of a follow-up study 6 years after a point source outbreak. Clin Infect Dis 2011 Apr 15;52(8):1013-9.

29. Million M, Thuny F, Richet $H$, Raoult $D$. Long-term outcome of $Q$ fever endocarditis: a 26year personal survey. Lancet Infect Dis 2010 Aug;10(8):527-35.

30. Raoult D, Tissot-Dupont H, Foucault C, Gouvernet J, Fournier PE, Bernit E, et al. Q fever 1985-1998. Clinical and epidemiologic features of 1,383 infections. Medicine (Baltimore) 2000 Mar;79(2):109-23.

31. Boyle B, Hone R. Q fever endocarditis revisited. Ir J Med Sci 1999 Jan;168(1):53-4.

32. Brouqui P, Dupont HT, Drancourt M, Berland Y, Etienne J, Leport C, et al. Chronic Q fever. Ninety-two cases from France, including 27 cases without endocarditis. Arch Intern Med 1993 Mar 8;153(5):642-8.

33. Fenollar F, Thuny $F$, Xeridat B, Lepidi $H$, Raoult D. Endocarditis after acute $Q$ fever in patients with previously undiagnosed valvulopathies. Clin Infect Dis 2006 Mar 15;42(6):818-21.

34. Bendermacher BL, Peppelenbosch AG, Daemen JW, Oude Lashof AM, Jacobs MJ. Q fever (Coxiella burnetii) causing an infected thoracoabdominal aortic aneurysm. J Vasc Surg 2011 May;53(5):1402-4.

35. Ellis ME, Smith CC, Moffat MA. Chronic or fatal Q-fever infection: a review of 16 patients seen in North-East Scotland (1967-80). Q J Med 1983;52(205):54-66.

36. Fournier PE, Casalta JP, Piquet P, Tournigand P, Branchereau A, Raoult D. Coxiella burnetii infection of aneurysms or vascular grafts: report of seven cases and review. Clin Infect Dis 1998 Jan;26(1):116-21.

37. Raoult D, Piquet $\mathrm{P}$, Gallais $\mathrm{H}$, de $\mathrm{MC}$, Drancourt M, Casanova P. Coxiella burnetii infection of a vascular prosthesis. N Engl J Med 1986 Nov 20;315(21):1358-9.

38. Sessa C, Vokrri L, Porcu P, Maurin M, Stahl JP, Magne JL. Abdominal aortic aneurysm and Coxiella burnetii infection: report of three cases and review of the literature. J Vasc Surg 2005 Jul;42(1):153-8.

39. Maurin M, Benoliel AM, Bongrand P, Raoult D. Phagolysosomes of Coxiella burnetiiinfected cell lines maintain an acidic pH during persistent infection. Infect Immun 1992 Dec;60(12):5013-6. 
40. Raoult D, Yeaman MR, Baca OG. Susceptibility of Coxiella burnetii to pefloxacin and ofloxacin in ovo and in persistently infected L929 cells. Antimicrob Agents Chemother 1989 May;33(5):621-3.

41. Raoult D, Drancourt M, Vestris G. Bactericidal effect of doxycycline associated with lysosomotropic agents on Coxiella burnetii in P388D1 cells. Antimicrob Agents Chemother 1990 Aug;34(8):1512-4.

42. Raoult D, Torres H, Drancourt M. Shell-vial assay: evaluation of a new technique for determining antibiotic susceptibility, tested in 13 isolates of Coxiella burnetii. Antimicrob Agents Chemother 1991 Oct;35(10):2070-7.

43. Rolain JM, Maurin M, Raoult D. Bacteriostatic and bactericidal activities of moxifloxacin against Coxiella burnetii. Antimicrob Agents Chemother 2001 Jan;45(1):301-2.

44. Sobradillo V, Zalacain R, Capelastegui A, Uresandi F, Corral J. Antibiotic treatment in pneumonia due to $Q$ fever. Thorax 1992 Apr;47(4):276-8.

45. Q-koorts, Dutch national guideline. 2010. (http://www.rivm.nl/dsresource?objectid=rivmp:6826\&type=org\&disposition=inline)

46. Delsing CE, Kullberg BJ. Q fever in the Netherlands: a concise overview and implications of the largest ongoing outbreak. Neth J Med 2008 Oct;66(9):365-7.

47. Delsing CE, Kullberg BJ, Bleeker-Rovers CP. Q fever in the Netherlands from 2007 to 2010. Neth J Med 2010 Dec;68(12):382-7.

48. Karagiannis I, Morroy G, Rietveld A, Horrevorts AM, Hamans M, Francken P, et al. Q fever outbreak in the Netherlands: a preliminary report. Euro Surveill 2007 Aug;12(8):E070809.

49. Schimmer B, Morroy G, Dijkstra F, Schneeberger PM, Weers-Pothoff G, Timen A, et al. Large ongoing $Q$ fever outbreak in the south of The Netherlands, 2008. Euro Surveill 2008 Jul 31;13(31).

50. Schimmer B, Dijkstra F, Vellema P, Schneeberger PM, Hackert V, ter SR, et al. Sustained intensive transmission of $Q$ fever in the south of the Netherlands, 2009. Euro Surveill 2009 May $14 ; 14(19)$.

51. van der Hoek W, Dijkstra F, Schimmer B, Schneeberger PM, Vellema P, Wijkmans C, et al. $Q$ fever in the Netherlands: an update on the epidemiology and control measures. Euro Surveill 2010 Mar 25;15(12).

52. van der Hoek W, Morroy G, Renders NH, Wever PC, Hermans MH, Leenders AC, et al. Epidemic Q fever in humans in the Netherlands. Adv Exp Med Biol 2012;984:329-64.

53. Schimmer B, Notermans DW, Harms MG, Reimerink JH, Bakker J, Schneeberger P, et al. Low seroprevalence of $Q$ fever in The Netherlands prior to a series of large outbreaks. Epidemiol Infect 2012 Jan;140(1):27-35.

54. Richardus JH, Schaap GJ, Donkers A, Dumas AM, Huisman J. [Q fever in the Netherlands; a description of 33 case reports observed between 1979 and 1983]. Ned Tijdschr Geneeskd 1984 Dec 1;128(48):2253-8.

55. Richardus JH, Dumas AM, Huisman J, Schaap GJ. Q fever in infancy: a review of 18 cases. Pediatr Infect Dis 1985 Jul;4(4):369-73.

56. Richardus JH, Donkers A, Dumas AM, Schaap GJ, Akkermans JP, Huisman J, et al. Q fever in the Netherlands: a sero-epidemiological survey among human population groups from 1968 to 1983. Epidemiol Infect 1987 Apr;98(2):211-9.

57. Boden K, Wagner-Wiening C, Seidel T, Baier M, Bischof W, Straube E, et al. Diagnosis of acute $Q$ fever with emphasis on enzyme-linked immunosorbent assay and nested poly- 
merase chain reaction regarding the time of serum collection. Diagn Microbiol Infect Dis 2010 Oct;68(2):110-6.

58. Fournier PE, Raoult D. Comparison of PCR and serology assays for early diagnosis of acute Q fever. J Clin Microbiol 2003 Nov;41(11):5094-8.

59. Schneeberger PM, Hermans MH, van Hannen EJ, Schellekens JJ, Leenders AC, Wever PC. Real-time PCR with serum samples is indispensable for early diagnosis of acute $Q$ fever. Clin Vaccine Immunol 2010 Feb;17(2):286-90.

60. Tilburg JJ, Melchers WJ, Pettersson AM, Rossen JW, Hermans MH, van Hannen EJ, et al. Interlaboratory evaluation of different extraction and real-time PCR methods for detection of Coxiella burnetii DNA in serum. J Clin Microbiol 2010 Nov;48(11):3923-7.

61. Curran T, Coulter WA, Fairley DJ, McManus T, Kidney J, Larkin M, et al. Development of a novel DNA microarray to detect bacterial pathogens in patients with chronic obstructive pulmonary disease (COPD). J Microbiol Methods 2010 Mar;80(3):257-61.

62. D'Harcourt SC, Soto AB, Burgos VC, Calero DL, Martinez-Zapico R. Comparison of immunofluorescence with enzyme immunoassay for detection of $Q$ fever. Eur J Clin Microbiol Infect Dis 1996 Sep;15(9):749-52.

63. Edlinger E. Immunofluorescence serology. A tool for prognosis of Q-fever. Diagn Microbiol Infect Dis 1985 Jul;3(4):343-51.

64. Embil J, Williams JC, Marrie TJ. The immune response in a cat-related outbreak of $Q$ fever as measured by the indirect immunofluorescence test and the enzyme-linked immunosorbent assay. Can J Microbiol 1990 Apr;36(4):292-6.

65. Field PR, Hunt JG, Murphy AM. Detection and persistence of specific IgM antibody to Coxiella burnetii by enzyme-linked immunosorbent assay: a comparison with immunofluorescence and complement fixation tests. J Infect Dis 1983 Sep;148(3):477-87.

66. Field PR, Santiago A, Chan SW, Patel DB, Dickeson D, Mitchell JL, et al. Evaluation of a novel commercial enzyme-linked immunosorbent assay detecting Coxiella burnetii-specific immunoglobulin $G$ for $Q$ fever prevaccination screening and diagnosis. J Clin Microbiol 2002 Sep;40(9):3526-9.

67. Fournier PE, Marrie TJ, Raoult D. Diagnosis of $Q$ fever. J Clin Microbiol 1998 Jul;36(7):1823-34.

68. Peter O, Dupuis G, Burgdorfer W, Peacock M. Evaluation of the complement fixation and indirect immunofluorescence tests in the early diagnosis of primary $Q$ fever. Eur J Clin Microbiol 1985 Aug;4(4):394-6.

69. Peter O, Dupuis G, Peacock MG, Burgdorfer W. Comparison of enzyme-linked immunosorbent assay and complement fixation and indirect fluorescent-antibody tests for detection of Coxiella burnetii antibody. J Clin Microbiol 1987 Jun;25(6):1063-7.

70. Swack NS, Gahan TF, Hausler WJ, Jr. The present status of the complement fixation test in viral serodiagnosis. Infect Agents Dis 1992 Aug;1(4):219-24.

71. Kovacova E, Kazar J, Spanelova D. Suitability of various Coxiella burnetii antigen preparations for detection of serum antibodies by various tests. Acta Virol 1998 Dec;42(6):365-8.

72. Slaba K, Skultety L, Toman R. Efficiency of various serological techniques for diagnosing Coxiella burnetii infection. Acta Virol 2005;49(2):123-7.

73. Peter O, Dupuis G, Peacock MG, Burgdorfer W. Comparison of enzyme-linked immunosorbent assay and complement fixation and indirect fluorescent-antibody tests for detection of Coxiella burnetii antibody. J Clin Microbiol 1987 Jun;25(6):1063-7. 
74. Dupont HT, Thirion X, Raoult D. Q fever serology: cutoff determination for microimmunofluorescence. Clin Diagn Lab Immunol 1994 Mar;1(2):189-96.

75. Dupuis G, Peter O, Luthy R, Nicolet J, Peacock M, Burgdorfer W. Serological diagnosis of Q fever endocarditis. Eur Heart J 1986 Dec;7(12):1062-6.

76. Villumsen S, Jorgensen CS, Smith B, Uldum S, Schiellerup P, Krogfelt KA. Determination of new cutoff values for indirect immunofluorescence antibody test for $Q$ fever diagnosis in Denmark. Diagn Microbiol Infect Dis 2009 Oct;65(2):93-8.

77. Dupuis G, Peter O, Luthy R, Nicolet J, Peacock M, Burgdorfer W. Serological diagnosis of Q fever endocarditis. Eur Heart J 1986 Dec;7(12):1062-6.

78. Dwyer DE, Gibbons VL, Brady LM, Cunningham AL. Serological reaction to Legionella pneumophila group 4 in a patient with Q fever. J Infect Dis 1988 Aug;158(2):499-500.

79. La SB, Raoult D. Serological cross-reactions between Bartonella quintana, Bartonella henselae, and Coxiella burnetii. J Clin Microbiol 1996 Sep;34(9):2270-4.

80. Lukacova M, Melnicakova J, Kazar J. Cross-reactivity between Coxiella burnetii and chlamydiae. Folia Microbiol (Praha) 1999;44(5):579-84.

81. Musso D, Raoult D. Serological cross-reactions between Coxiella burnetii and Legionella micdadei. Clin Diagn Lab Immunol 1997 Mar;4(2):208-12.

82. Numazaki K, Ueno H, Yokoo K, Muramatsu Y, Chiba S, Morita C. Detection of serum antibodies to Bartonella henselae and Coxiella burnetii from Japanese children and pregnant women. Microbes Infect 2000 Oct;2(12):1431-4.

83. Schramek S, Kazar J, Sadecky E. Serological cross-reactions of lipid A components of lipopolysaccharides isolated from Chlamydia psittaci and Coxiella burnetii. Acta Virol 1980 May;24(3):224.

84. Tsuneoka H, Ouchi K, Nagaoka H, Ishida C, lino H, Murakami K, et al. [Serological crossreaction among Bartonella henselae, Chlamydia pneumoniae and Coxiella burnetii by indirect fluorescence antibody method]. Kansenshogaku Zasshi 2001 May;75(5):406-10.

85. Public Health Act. 2008. (http://wetten.overheid.nl/BWBR0024705)

86. Dupuis G, Peter O, Luthy R, Nicolet J, Peacock M, Burgdorfer W. Serological diagnosis of Q fever endocarditis. Eur Heart J 1986 Dec;7(12):1062-6.

87. Raoult $D$, Marrie T, Mege J. Natural history and pathophysiology of $Q$ fever. Lancet Infect Dis 2005 Apr;5(4):219-26.

88. Fournier PE, Casalta JP, Habib G, Messana T, Raoult D. Modification of the diagnostic criteria proposed by the Duke Endocarditis Service to permit improved diagnosis of $Q$ fever endocarditis. Am J Med 1996 Jun;100(6):629-33.

89. Li JS, Sexton DJ, Mick N, Nettles R, Fowler VG, Jr., Ryan T, et al. Proposed modifications to the Duke criteria for the diagnosis of infective endocarditis. Clin Infect Dis 2000 Apr;30(4):633-8.

90. Dupuis G, Peter O, Luthy R, Nicolet J, Peacock M, Burgdorfer W. Serological diagnosis of Q fever endocarditis. Eur Heart J 1986 Dec;7(12):1062-6.

91. Peacock MG, Philip RN, Williams JC, Faulkner RS. Serological evaluation of $O$ fever in humans: enhanced phase I titers of immunoglobulins $G$ and $A$ are diagnostic for $Q$ fever endocarditis. Infect Immun 1983 Sep;41(3):1089-98.

92. Peter O, Flepp M, Bestetti G, Nicolet J, Luthy R, Dupuis G. Q fever endocarditis: diagnostic approaches and monitoring of therapeutic effects. Clin Investig 1992 Oct;70(10):932-7.

93. Soriano F, Camacho MT, Ponte C, Gomez P. Serological differentiation between acute (late control) and endocarditis Q fever. J Clin Pathol 1993 May;46(5):411-4. 
94. Worswick D, Marmion BP. Antibody responses in acute and chronic $Q$ fever and in subjects vaccinated against $Q$ fever. J Med Microbiol 1985 Jun;19(3):281-96.

95. Camacho MT, Outschoorn I, Echevarria C, Kovacova E, Yebra M, Mate I, et al. Distribution of IgA subclass response to Coxiella burnetii in patients with acute and chronic $Q$ fever. Clin Immunol Immunopathol 1998 Jul;88(1):80-3.

96. Fournier PE, Raoult D. Predominant immunoglobulin A response to phase II antigen of Coxiella burnetii in acute $Q$ fever. Clin Diagn Lab Immunol 1999 Mar;6(2):173-7.

97. Rolain JM, Lecam C, Raoult D. Simplified serological diagnosis of endocarditis due to Coxiella burnetii and Bartonella. Clin Diagn Lab Immunol 2003 Nov;10(6):1147-8.

98. Wegdam-Blans MC, Nabuurs-Franssen MN, Horrevorts AM, Peeters MF, Schneeberger PM, Bijlmer HA. [Laboratory diagnosis of acute Q fever]. Ned Tijdschr Geneeskd 2010;154:A2388. 




\section{Chapter 2}

\section{Evaluation of commonly used serological tests for the detection of Coxiella burnetii antibodies in well-defined acute and follow-up sera}

Clinical and Vaccine Immunology 2012; 19(7): 1110-5

M.C.A. Wegdam-Blans C.C.H. Wielders

J. Meekelenkamp J.M. Korbeeck

T. Herremans H.T. Tjhie H.A. Bijlmer M.P.G. Koopmans P.M. Schneeberger 


\section{Abstract}

In this study we compared an Indirect Fluorescent Antibody Test (IFAT) (Focus Diagnostics), a commercially available Enzyme-linked Immunosorbent Assay (ELISA) (Virion/Serion) and a Complement Fixation Test (CFT) (Virion/Serion) for the detection of Coxiella burnetii IgG phase I, IgG phase II and IgM phase II. For this, we used a unique collection of acute and convalescent sera from 126 patients with acute $Q$ fever diagnosed by positive Coxiella burnetii PCR in blood. We were able to establish a reliable date of onset of disease as DNA is detectable within two weeks after the start of symptoms. In acute samples, at $\mathrm{t}=0$, IFAT demonstrated IgM phase II antibodies in significantly more sera compared to ELISA (31.8\% versus $19.7 \%$ ), although the portion of solitary IgM phase II was equal for IFAT and for ELISA (18.2\% and $16.7 \%$, respectively). Twelve months after the diagnosis of acute $Q$ fever, $83.5 \%$ and $62.2 \%$ of the sera were still positive for IgM phase II with IFAT and ELISA, respectively. Therefore, definitive serological evidence of acute $Q$ fever cannot be based on IgM in a single serum sample in epidemic or endemic areas where infections are common. At 12 months IFAT IgG phase II showed the slowest decline. Based on IgG phase II antibody detection in paired samples (at 0 and 3 months) from 62 patients, IFAT confirmed more cases than ELISA and CFT, but the differences were not statistically significant (100\% IFAT, 95.2\% ELISA and 96.8\% CFT).

This study demonstrated that the three serological tests are equally effective in diagnosing acute $Q$ fever within three months of start of symptoms. In follow-up sera, more IgG antibodies were detected by IFAT than by ELISA or CFT, making IFAT more suitable for pre-vaccination screening programs. 


\section{Introduction}

$Q$ fever is a zoonotic disease and human infections result mainly from inhalation of Coxiella burnetii (C. burnetii) contaminated aerosols [14]. A major goat-related epidemic in the Netherlands, starting in 2007, resulted in more than 4,000 acute $Q$ fever cases notified in the national infectious disease notification system $[3,6,13]$. The epidemic was brought under control through targeted vaccination and culling of goats, resulting in a sharp decline of cases in 2010 [3]. Clinical manifestations occur in about half of the acute $Q$ fever cases after an incubation period of two to four weeks [14]. Moreover, clinical symptoms may be indiscriminative and vary from flu-like illness to a more severe syndrome with high fever, severe headache, gastrointestinal complaints, pneumonia and hepatitis [14].

Laboratory diagnosis of acute $Q$ fever is ideally based on a combination of PCR and serology in blood [16]. PCR has been shown to be positive in almost all early acute $Q$ fever patients that have not yet mounted an antibody response and in almost all of those that have just mounted an IgM phase II antibody response [16]. Conversely, PCR becomes negative in patients that have developed IgG antibodies [16]. However, the laboratory diagnosis of an acute $C$. burnetii infection in routine practice is mainly based on serology, because the majority of samples will not be submitted to the laboratory within two weeks after onset of the disease. IgM phase II is the first antibody to be detected in blood, followed by IgG phase II [5]. The serologic diagnosis of acute $Q$ fever based on a single serum sample can be inaccurate, as positive IgM phase II may persist for a longer period and solitary IgM phase II can be false positive $[15,16]$. Therefore, seroconversion or a fourfold increase in IgG phase II titer is recommended to confirm the diagnosis of acute $Q$ fever [5]. There are different serological tests available for $Q$ fever, including Indirect Fluorescent Antibody Tests (IFAT), Enzyme-linked Immunosorbent Assays (ELISA) and Complement Fixation Tests (CFT). In the Netherlands, stringent criteria were developed to support clinical decisionmaking, based on our observations and the different serological test outcomes during the epidemic, including an algorithm that includes confirmatory testing $[15,18]$. Briefly, this algorithm comprises the use of PCR and serology tests, where the choices for first line assay depends on the time between the first day of illness and the serum collection: for patients sampled within the first two weeks of illness, it is recommended to perform PCR. For patients 
with first contact with a physician later than two weeks post illness onset, or for patients for whom the date of illness onset was not known, serology is recommended as initial test. The aim of the present study was to compare the three serological assays in a large number of acute and sequential convalescent serum samples from a patient group with acute $Q$ fever, from which onset of disease could be estimated within three weeks, as all of them had been diagnosed with $Q$ fever through positive PCR. In order to assess applicability for seroprevalence studies we compared the diagnostic performance of different tests for acute disease, as well as the kinetics in sequential serum samples.

\section{Materials and Methods}

\section{Case definition}

Acute $Q$ fever was diagnosed based on positive $C$. burnetii PCR (NucliSENS easyMAG, bioMérieux, Boxtel, the Netherlands) in peripheral blood combined with clinical symptoms consistent with acute $Q$ fever syndrome, and in absence of signs and symptoms of chronic $Q$ fever.

\section{Serum samples}

Patients diagnosed with acute $Q$ fever during the $Q$ fever epidemic were routinely monitored at 3,6 and 12 months after diagnosis using IFAT. For the purpose of this study, all sera were retested with ELISA and CFT. A total of 126 patients, diagnosed between March and December 2009, were included (433 serum samples), with the following distribution of samples per time point: 66 at $\mathrm{t}=0$ (time of diagnosis), 121 at $\mathrm{t}=3$ months, 121 at $\mathrm{t}=6$ months, and 125 at $\mathrm{t}=12$ months after diagnosis. Paired sera at time points 0 and 3 months were available for 62 acute $Q$ fever patients. Age, sex and laboratory results for all patients were extracted from the hospital database, and the data was made anonymous for use in further analysis.

\section{IFAT (Focus Diagnostics, Cypress, USA)}

IgG phase I and II, and IgM phase I and II were measured according to the manufacturer's instructions with the exception of a dilution scheme: serum samples were two-fold diluted starting with $1: 32$ by adding $5 \mu$ l of serum to $155 \mu$ l of PBS. First, single dilution of 1:32 were viewed using a fluorescence microscope (at a magnification of 400x). Positive samples were further dilut- 
ed in this test to quantify an endpoint. Whenever sera were still positive after eight dilutions (1:4,096), titers were set at 8,192.

\section{CFT (Virion/Serion, Würzburg, Germany)}

Phase I and II were measured according to the manufacturer's instructions. After the procedure steps, ready-to-use hemolytic system (Virion/Serion, Germany) was used before measuring the titers by eye. Dilutions with $100 \%$ of hemolysis were defined as positive. A positive result was defined as having an endpoint dilution of $\geq 1: 8$. Titers were set at 1,024 when sera were still positive after eight dilutions (1:512).

\section{ELISA (Virion/Serion, Würzburg, Germany)}

IgG phase I and II, and IgM phase II were processed on a fully automated 4plate ELISA processing system (DSX). Different dilution protocols were used according to the manufacturer's instructions, using 1:100 dilution for IgG phase I and IgM phase II and 1:500 dilution for IgG phase II assay. Data were analyzed according to the Virion/Serion protocol, reporting IgG phase I and IgM phase II qualitatively and IgG phase II quantitatively. IgG phase I and IgG M phase II were positive whenever the measured absorbance was more than $10 \%$ above the extinction of the cut-off control. Ambiguous results were added to negative results. IgG phase I extinctions were expressed in OD values. IgG phase II extinctions were expressed in $\mathrm{U} / \mathrm{ml}$ titer using a logistic-log-model calculation and were defined as positive when titer $>30$ $\mathrm{U} / \mathrm{ml}$.

\section{Data analysis}

Statistical analysis included computations of frequencies and analysis of agreement between ELISA, IFAT and CFT. McNemar exact tests with a binomial distribution were used to test for significant differences between the frequencies obtained with the three diagnostic tests. Spearman's rank correlation was used to investigate the agreement between the tests at time point 3. To investigate the kinetics of IgG phase II using the different methods, ratios of the titers between successive time points were examined. Ratios were calculated by dividing the value at 6 months by that of 3 months $(6 / 3)$ and that of 12 months by 6 months (12/6) for each patient. This was carried out for each method and the average ratio was calculated afterwards. A ratio above 1 was in accordance with a rise in titer and below 1 was in accord- 
ance with a decline in titer. Significance of ratio differences between the methods and time points were pair-wise computed using two sample t-tests.

\section{Results}

\section{Patients' characteristics}

Mean age $( \pm S D)$ of the 126 patients was $51.2( \pm 15.3)$ years and 83 patients $(65.9 \%)$ were male. For the 62 acute $Q$ fever patients with paired sera available at time points 0 (all PCR positive sample) and 3 months, mean age was $53.3( \pm 15.0)$ years and 40 patients $(64.6 \%)$ were male.

\section{Early serology results ( 0 and 3 months)}

At $\mathrm{t}=0$, when all samples were PCR positive, IgM phase II antibodies were more frequently detected with IFAT compared to ELISA $(31.8 \%(21 / 66)$ and $19.7 \%(13 / 66)$ of patients, respectively $(p=0.008)$ ) (figure 1). IgG phase II was positive in $13.6 \%(9 / 66)$ and $3.0 \%$ (2/66) for IFAT and ELISA, respectively $(p=0.016)$. This means that the solitary IgM phase II antigen response was equal in IFAT as well as in ELISA (18.2\% and $16.7 \%$ for IFAT and ELI$\mathrm{SA}$, respectively (difference not significant)). Based on IgG phase II antibody detection in paired samples (at 0 and 3 months) from 62 patients, IFAT confirmed more cases than ELISA and CFT, but the differences were not statically significant: $100 \%$ using IFAT, $95.2 \%$ using ELISA and $96.8 \%$ using CFT (IFAT vs ELISA: $p=0.250$; IFAT vs CFT: $p=0.500$; ELISA vs CFT: $\mathrm{p}=1.000)$ (table 1).

\section{IgM phase II}

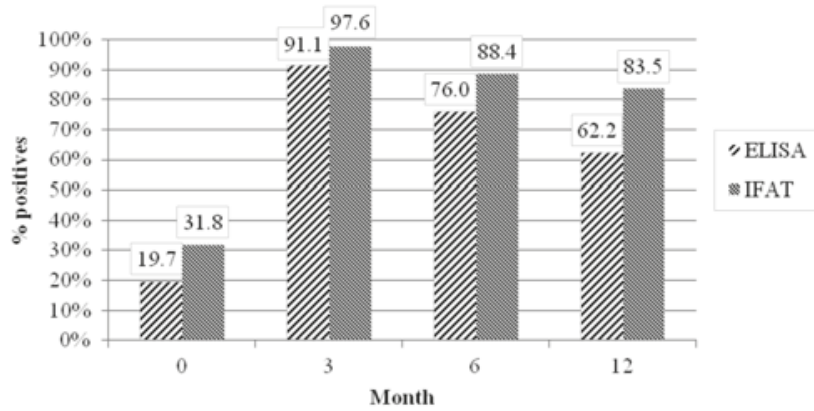

Figure 1. Performance of $Q$ fever IgM phase II testing using ELISA and IFAT at different time points (percentages are indicated above the bars). Significant differences between IFAT and ELISA were measured at time points $0,3,6$, and 12 months $(P=0.008, P=0.021, P=0.001$, and $P=0.000$, respectively). 


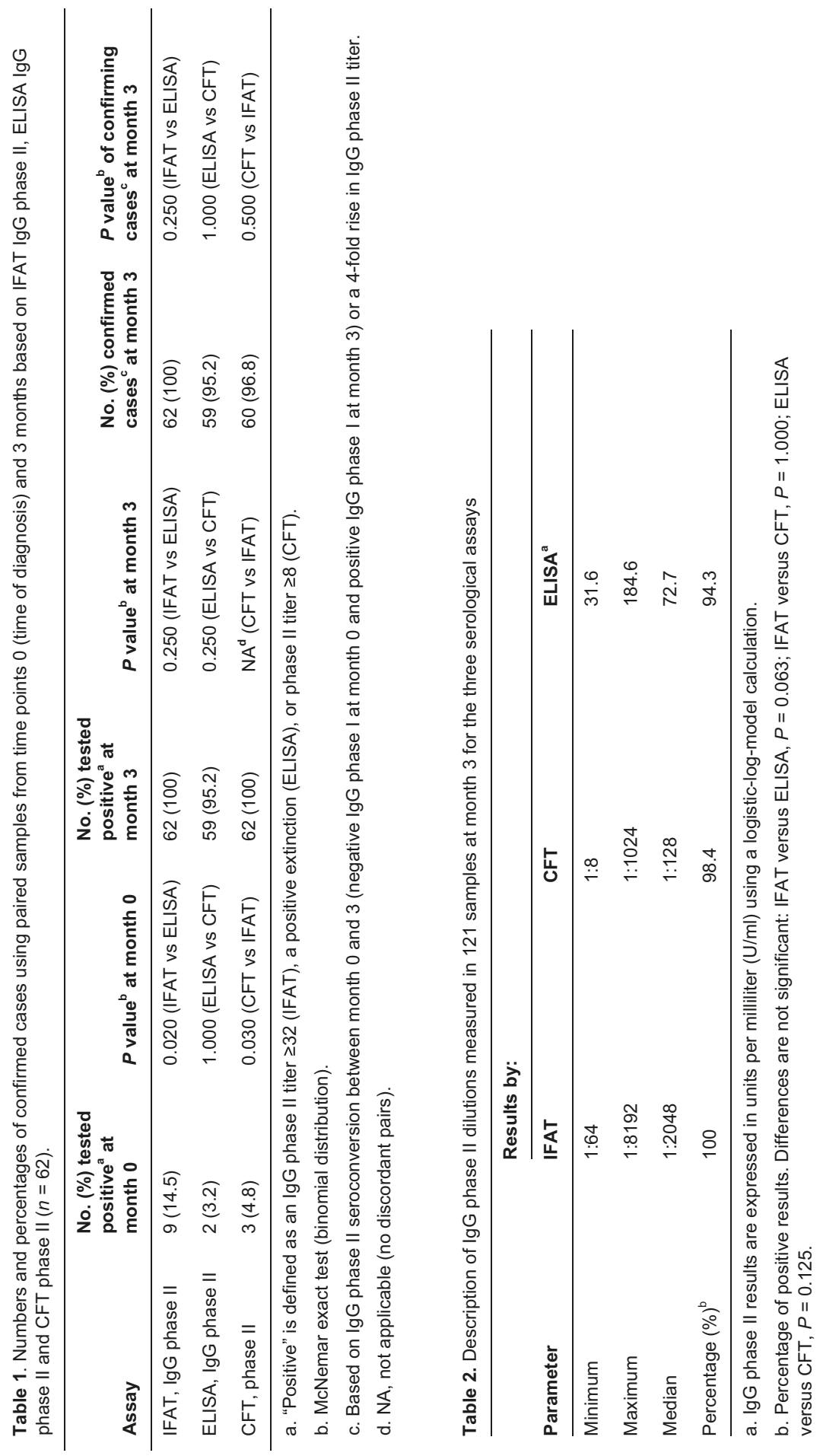


Figure 2 A-C shows the comparison of ELISA and IFAT IgG phase II, and CFT phase II at $\mathrm{t}=3$ months. Agreement between IFAT and CFT, expressed in the Spearman's rank correlation coefficient, was better compared to agreements between IFAT and ELISA or CFT and ELISA at $t=3$ months (CFT vs IFAT: $r=0.85$; IFAT vs ELISA: $r=0.45$; CFT vs ELISA: $r=0.34$ ). At $t=3$ months, almost all 121 samples tested highly positive for IgG phase II: median titer was 2048, 128 and 72.7 for IFAT, CFT and ELISA respectively (table 2). The percentage IgG phase II test results was higher using IFAT or CFT compared to ELISA, but this difference was not statistically significant.

A

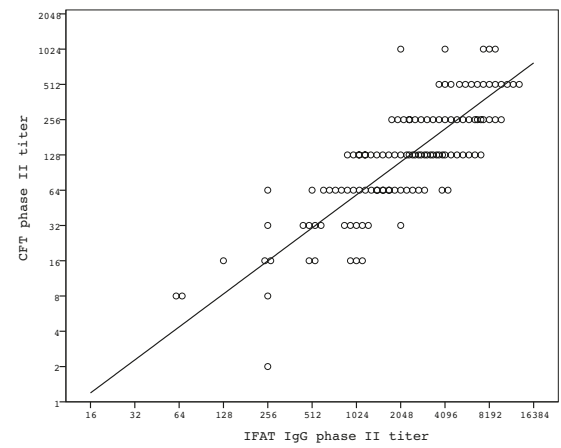

C

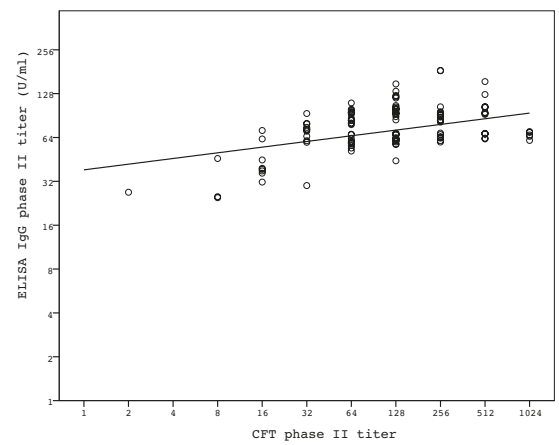

B

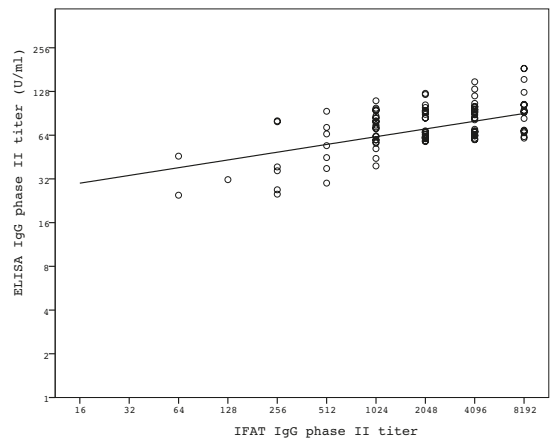

Figure 2. (A to $\mathbf{C}$ ) Logarithmic (2-log) relation between IgG phase II tests at time point 3 months. (A) IFAT and CFT; Spearman's rank correlation $r=0.85$. (B) IFAT and ELISA; $r=0.45$. (C) CFT and ELISA; $r=0.34$. (IFA IgG phase II titer of 16 equals IFA IgG phase II titer of <32; IFA IgG phase II titer of 8,192 equals IFA IgG phase II titer of $>4,096$; CFT phase II titer of 2 equals CFT phase II titer of $<4$; CFT phase II titer of 1,024 equals CFT phase II titer of $>512$ ). 


\section{Late serology results (6 and 12 months)}

At $\mathrm{t}=6$ and $\mathrm{t}=12$ months, IgM antibodies persisted in a high proportion of patients, but this differed depending on the test used (83.5\% vs $62.2 \%$ of patients IgM phase II positive at $\mathrm{t}=12$ by IFAT and ELISA, respectively $(p=0.000)$ ) (figure1)). Although declining, a substantial part of the IgM phase II titers measured with IFAT remained highly positive even at 12 months (table 3). The time kinetics of IgG antibodies are shown in figure $\mathbf{3}$ and 4. IgG phase II and IgG phase I were both significantly more often detected with IFAT in follow-up sera (figure 3 ). IFAT showed no decline (ratio $>1$ ) during the 12 months of follow-up, compared to ELISA and CFT. The IFAT $6 / 3$ ratio is significantly different compared to CFT and ELISA, $p=0.023$ and $p=0.002$, respectively, as well as the IFAT $12 / 6$ ratio, $p=0.005$ and $p=0.000$. This means that IgG phase II titers measured with IFAT faded less rapidly after acute infection, compared to ELISA and CFT- measured titers (figure 4).

Table 3. Percentages of samples with negative IFAT IgM phase II, low-positive IgM phase II and highpositive IgM phase II results

\begin{tabular}{llll}
\hline & \multicolumn{3}{l}{$\%$ of samples with IgM phase II result (endpoint dilution) } \\
\cline { 2 - 4 } Time point & $\begin{array}{l}\text { Negative } \\
(<1: 32)\end{array}$ & $\begin{array}{l}\text { Low positive } \\
(\mathbf{1 : 3 2 - 1 : 2 5 6 )}\end{array}$ & $\begin{array}{l}\text { High positive } \\
(>1: 256)\end{array}$ \\
\hline Month 0 & 71.4 & 23.8 & 4.8 \\
Month 3 & 1.7 & 15.7 & 82.6 \\
Month 6 & 11.6 & 34.7 & 53.7 \\
Month 12 & 16.0 & 64.0 & 20.0
\end{tabular}

${ }^{a}$ Two positive samples were not diluted further because sera were lost. Therefore, percentages are based on 120 sera.

Differences in IgG phase I detection in follow-up sera were remarkable. Significant differences between tests were observed at all of the time points, except $t=0$. At $t=3$ months, IgG phase I was significantly more often positive with IFAT compared to ELISA or CFT; IFAT 80.2\% (97/121), ELISA $8.3 \%$ (10/121), CFT $7.4 \%$ (9/121) (IFAT vs ELISA and IFAT vs CFT: $p=0.000$; ELISA vs CFT: $p=1.000)$. At $t=6$ months, the same difference was observed, except that in addition ELISA and CFT differences were statistically significant: IFAT 86.8\% (105/121), ELISA 33.9\% (41/121), CFT 24.0\% (29/121) (IFAT vs ELISA and IFAT vs CFT: $p=0.000$; ELISA vs CFT: $p=0.008$ ). $T=12$ months showed differences comparable to $t=6$ months: IFAT $76.0 \%$ 
(95/125), ELISA $28.0 \%(35 / 125)$, and CFT $13.6 \%(17 / 125)(p=0.000$ for all three combinations of tests) (figure 3 ).

A

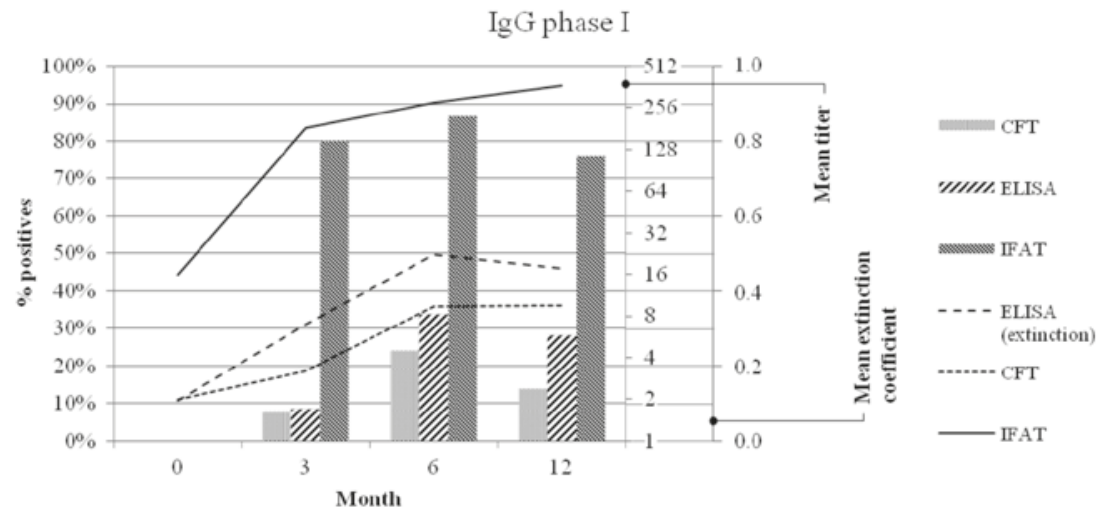

B

IgG phase II

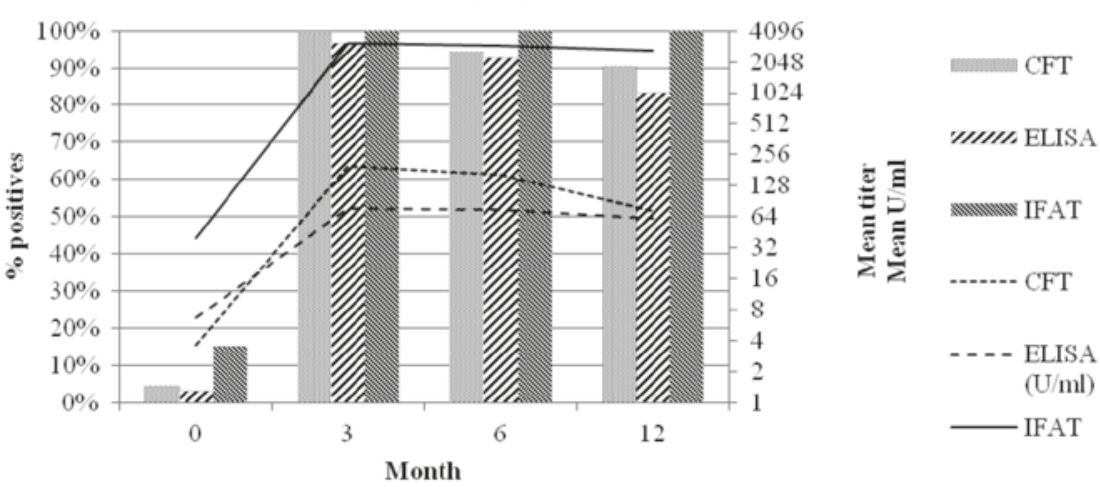

Figure 3. (A) Overview of IgG phase I detection with IFAT, ELISA, and CFT at time points 0,3 , 6, and 12 months. Bars represent percentages of positive results (left axis), and lines represent mean titers or mean extinction coefficients (both right axis). (B) Overview of IgG phase II detection with IFAT, ELISA, and CFT at the same time points. Bars represent percentages of positive results (left axis), and lines represent mean titers or mean $\mathrm{U} / \mathrm{ml}$. IgG phase II ELISA results are expressed in $\mathrm{U} / \mathrm{ml}$ using a 2-log calculation model and therefore can be plotted at the same axis as those of IFAT. 


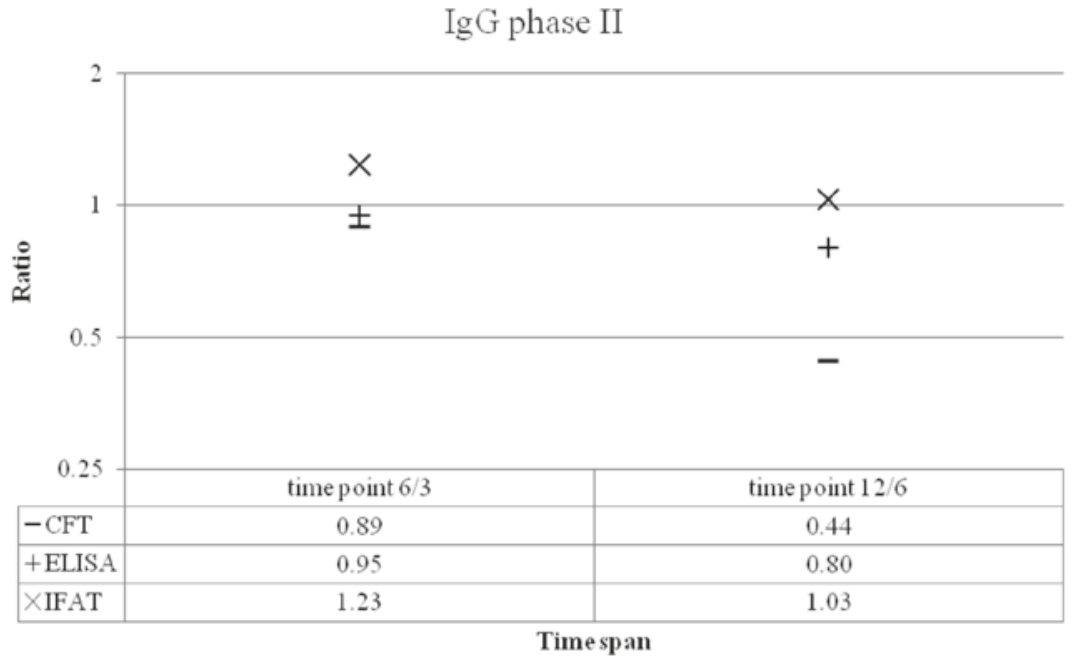

Figure 4. Average IgG phase II ratios at different time points for IFAT, ELISA, and CFT. A ratio above 1 is in accordance with a rise, and a ratio below 1 is in accordance with a decline, in titer.

\section{Discussion}

In this present study, we used a unique very well-defined acute $Q$ fever patient group, based on positive PCR in acute sera. As detection of $C$. burnetii DNA in serum with PCR is highly time-dependent, being almost negligible approximately two weeks after onset of acute $Q$ fever symptoms, the date of onset of disease in this serum collection could be calculated within two weeks. Due to this short period of DNA detection time, serological tests will always be necessary in diagnostic algorithms. Besides, not all diagnostic laboratories have $\mathrm{Q}$ fever PCR facilities available.

Based on the comparison of three serological tests, IFAT (Focus Diagnostics), ELISA (Virion/Serion) and CFT (Virion/Serion) at onset of disease and at 3, 6 and 12 months after positive PCR, conclusions can be drawn on the concordance and the values of these assays in the diagnosis of acute and past $Q$ fever infection.

IgM phase II antibodies are the first to be detected in acute $Q$ fever, after detection of $C$. burnetii DNA in serum [5]. There is an overlap between the disappearance of DNA and the detection of IgM phase II antibodies [11, 16]. In our study this overlap is more pronounced with IFAT than with ELISA: IgM phase II measured with IFAT and ELISA was $31.8 \%$ and $19.7 \%$, respective- 
ly. At 12 months IgM antibodies persisted both with IFAT (83.5\%) and ELISA $(62.2 \%)$. In earlier reports IFAT IgM phase II dilution cut-off $\geq 1: 50$ was proposed as a sole diagnostic marker of acute $Q$ fever and it was shown that IgM titers declined to undetectable levels within four months [5, 8, 14]. However in our study, IgM phase II antibody was still detectable in a major part of the 12 months' sera with significant titers, which is consistent with a previous study showing persisting IgM antibodies in late follow-up serum samples [7]. We showed that IgM phase II and IgG phase II can persist for up to a year and probably longer, also the decline in IgG phase II titers is slow, especially with IFAT. This means that serum samples with isolated IgM phase II or samples with low IgM and IgG phase II titers, diagnosis of acute $Q$ fever should be confirmed with a second serum sample yielding significant high titers [12, 16]. In contrast, if a first serum sample already shows high titers of both IgM and IgG phase II no definitive conclusions can be drawn, as this may be both compatible with acute infection and past infection. The predictive values of single serological results could be altered by changing cut-off values but this highly depends on the incidence and prevalence of $Q$ fever in a certain area in a certain period. For instance in the south of the Netherlands in 2007 and 2008, when there was a low prevalence and a moderate incidence [6], low titers would most likely be associated with acute $Q$ fever (or with an aspecific result). In 2009 when there was an incredibly high incidence, most low titers would be associated with acute $Q$ fever [12]. Then from 2010 and onwards, again a period of low incidence, low titers would most likely be associated with past infections, because of the high prevalence of antibodies due to the epidemic [6]. In our opinion, definitive serological evidence of acute $Q$ fever cannot be based on a single serum sample only, but should involve measurement of both $\lg \mathrm{M}$ and $\lg \mathrm{G}$ antibodies in paired serum samples.

In the present study, no significant differences were found between IFAT, CFT and ELISA in confirming acute $Q$ fever in 62 paired sera within three months, based on IgG phase II. There was a moderate to good agreement between the tests regarding the level of titers, with IFAT and CFT showing the closest agreement. Hence, choosing one serological technique above another could be based on practical preferences, but the differences in test performances showed in this study should be taken into account too. IFAT and CFT are more laborious techniques and interpretation (especially with regard to IFAT) can be subjective. IFAT is the reference method for serolog- 
ical diagnosis as it can distinguish between acute, past and chronic infection [14]. Finally, ELISA is easy to perform and is adapted for automation. Our results are in agreement with previous studies, but those were all limited by small sample sizes and the variability in the definition of acute $Q$ fever $[2,4$, 9, 17].

Significant differences were demonstrated in follow-up sera in IgG phase I and phase II antibody detection. Decline of IgG phase II antibody titers was slower with IFAT (figure 4), and IgG phase II antibodies were significantly more often detected with IFAT after one year (figure 3), compared to ELISA and CFT. It is likely that these phenomena continue longer than 12 months. This makes IFAT the most preferable method in studies where high sensitivity is essential, like vaccination strategies, where false negative results should be avoid. Alternatively, IFAT could be used in combination with ELISA, for example in case of large amount of samples: ELISA as the primary screening method and IFAT for retesting ELISA negative results $[1,10]$. Differences in IgG phase I antibodies were most interesting, with significantly more positive titers using IFAT, although low range dilutions (1:32 $1: 128)$ were overrepresented in our IFAT results. As far as we know, this is the first report of the comparison of IgG phase I using different serological methods in follow-up sera. Unfortunately we were unable to address the issue of chronic $Q$ fever diagnosis because of the sample size and inclusion criteria of this study.

Due to the selected dataset, no conclusions can be drawn concerning the sensitivity and specificity of the assays in this study. Moreover, because the interval of the first convalescent sample was set at month 3 , this study does not contain data on the intermediate period. In this intermediate period analysis of cut-off values could be possible: lower cut-off values could be used to screen for positives which still needs to be confirmed with a titer rise to exclude past infections or aspecific reactions. Another drawback of this study is the limited follow-up period. Differences in decline rates of IgG phase II could only be analyzed for a period of one year. Further studies are needed to confirm whether these differences will persist over a longer period.

In conclusion, in this clearly defined acute $Q$ fever patient cohort, we demonstrated that confirmation of acute $Q$ fever within three months is reliable and independent of the test method used. IgM phase II is not often solitary positive early in the disease but is still detectable in a major part of the samples at 12 months, especially with IFAT. This complicates the diagnosis 
in epidemic and endemic regions making paired sera necessary to confirm the diagnosis by a significant (fourfold) titer rise in IgG phase II. IgG phase I and II antibodies in follow-up sera were detected significantly more often with IFAT, compared to CFT and ELISA, making IFAT more suitable for vaccination strategies. 


\section{References}

1. Blaauw G.J., D.W. Notermans, B. Schimmer, J. Meekelenkamp, J.H. Reimerink, P. Teunis, and P.M. Schneeberger. 2012. The application of an enzyme-linked immunosorbent assay or an immunofluorescent assay test leads to different estimates of seroprevalence of Coxiella burnetii in the population. Epidemiol. Infect. 140:36-41.

2. Boden K., C. Wagner-Wiening, T. Seidel, M. Baier, W. Bischof, E. Straube, and P. Kimmig. 2010. Diagnosis of acute $Q$ fever with emphasis on enzyme-linked immunosorbent assay and nested polymerase chain reaction regarding the time of serum collection. Diagn. Microbiol. Infect. Dis. 68:110-116.

3. Delsing, C. E., B. J. Kulberg, and C. P. Bleeker-Rovers. 2010. Q fever in the Netherlands from 2007 to 2010. Neth. J. Med. 68:382-387.

4. D'Harcourt S. C., A. B. Soto, V. C. Burgos, D. L. Calero, and R. Martínez-Zapico. 1996. Comparison of immunofluorescence with enzyme immunoassay for detection of $Q$ fever. Eur. J. Clin. Microbiol. Infect. Dis. 15:749-752.

5. Dupuis G., O. Péter, M. Peacock, W. Burgdorfer, and E. Haller. 1985. Immunoglobulin responses in acute $Q$ fever. J. Clin. Microbiol. 22:484-487.

6. Dijkstra F., W. van der Hoek, N. Wijers, B. Schimmer, A. Rietveld, C.J. Wijkmans, P. Vellema, and P.M. Schneeberger. 2012. The 2007-2010 Q fever epidemic in The Netherlands: characteristics of notified acute $Q$ fever patients and the association with dairy goat farming. FEMS Immunol. Med. Microbiol. 64;3-12.

7. Embil J., J. C. Williams, and T. J. Marrie. 1990. The immune response in a cat-related outbreak of $Q$ fever as measured by the indirect immunofluorescence test and the enzyme-linked immunosorbent assay. Can. J. Microbiol 36:292-296.

8. Field P. R., J. G. Hunt, and A. M. Murphy. 1983. Detection and persistence of specific IgM antibody to Coxiella burnetii by enzyme-linked immunosorbent assay: a comparison with immunofluorescence and complement fixation tests. J. Infect. Dis. 148:477-487.

9. Field P. R., J. L. Mitchell, A. Santiago, D. J. Dickeson, S. W. Chan, D. W. Ho, A. M. Murphy, A. J. Cuzzubbo, and P. L. Devine. 2000. Comparison of a commercial enzyme-linked immunosorbent assay with immunofluorescence and complement fixation tests for detection of Coxiella burnetii (Q fever) immunoglobulin M. J. Clin. Microbiol. 38:1645-1647.

10. Field P. R., A. Santiago, S. W. Chan, D. B. Patel, D. Dickeson, J. L. Mitchell, P. L. Devine, and A. M. Murphy. 2002. Evaluation of a novel commercial enzyme-linked immunosorbent assay detecting Coxiella burnetii-specific immunoglobulin $\mathrm{G}$ for $\mathrm{Q}$ fever prevaccination screening and diagnosis. J. Clin. Microbiol. 40:3526-3529.

11. Fournier P. E. and D. Raoult. 2003. Comparison of PCR and serology assays for early diagnosis of acute $Q$ fever. J. Clin. Microbiol. 41:5094-5098.

12. Jager M.M., G. Weers-Pothoff, M.H. Hermans, J.C. Meekelenkamp, J.J. Schellekens, N.H. Renders, A.C. Leenders, P.M. Schneeberger, and P.C. Wever. 2011. Evaluation of a diagnostic algorithm for acute $Q$ fever in an outbreak setting. Clin. Vaccine. Immunol. 18:963-968.

13. Karagiannis I., B. Schimmer, A.Van Lier, A. Timen., P. Schneeberger, B. Van Rotterdam, A. De Bruin, C. Wijkmans, A. Rietveld, and Y.Van Duynhoven. 2009. Investigation of a $Q$ fever outbreak in a rural area of The Netherlands. Epidemiol. Infect. 137:1283-1294.

14. Maurin, M., and D. Raoult. Q fever. 1999. Clin. Microbiol. Rev. 12:518-553. 
15. Raven C.F., J.L. Hautvast, T. Herremans, A.C. Leenders, and P.M. Schneeberger. 2012. Solitary IgM phase II response has a limited predictive value in the diagnosis of acute $Q$ fever. Epidemiol Infect. 20:1-5.

16. Schneeberger P. M., M. H. Hermans, E. J. Van Hannen, J. J. Schellekens, A. C. Leenders, and P. C. Wever. 2010. Real-time PCR with serum samples is indispensable for early diagnosis of acute $Q$ fever. Clin. Vaccine Immunol. 17:286-290.

17. Uhaa I. J., D. B. Fishbein, J. G. Olson, C. C. Rives, D. M. Waag, and J. C. Williams. 1994. Evaluation of specificity of indirect enzyme-linked immunosorbent assay for diagnosis of human Q fever. J. Clin. Microbiol. 32:1560-1565.

18. Wegdam-Blans M.C.A., M.N. Nabuurs-Franssen, A.M. Horrevorts, M.F. Peeters, P.M. Schneeberger, and H.A. Bijlmer. 2010. Laboratory diagnosis of acute $Q$ fever. Ned Tijdschr Geneeskd. 154:A2388. 




\section{Chapter 3}

\section{A dynamic case definition is warranted for adequate notification in an extended epidemic setting: the Dutch Q fever outbreak 2007-2009 as exemplar}

Eurosurveillance 2013;18(41): pii=20606

G. Jaramillo-Gutierrez

M.C.A. Wegdam-Blans

R. ter Schegget

J.M. Korbeeck

R. van Aken

H.A. Bijlmer

H.T. Tjhie

M.P.G. Koopmans 


\section{Abstract}

$Q$ fever is a notifiable disease in the Netherlands: laboratories are obliged to notify possible cases to the Municipal Health Services. These services then try to reconfirm cases with additional clinical and epidemiological data and provide anonymised reports to the national case register of notifiable diseases. Since the start of the 2007-2009 Dutch Q fever outbreak, notification rules remained unchanged, despite new laboratory insights and altered epidemiology. In this study, we retrospectively analysed how these changes influenced the proportion of laboratory-defined acute $Q$ fever cases (confirmed, probable and possible) that were included in the national case register, during (2009) and after the outbreak (2010 and 2011). The number of laboratory-defined cases notified to the Municipal Health Services was 377 in 2009, 96 in 2010 and 50 in 2011. Of these, 186 (49.3\%) in 2009, 12 $(12.5 \%)$ in 2010 and $9(18.0 \%)$ in 2011 were confirmed as acute infection by laboratory interpretation. The proportion of laboratory-defined acute $Q$ fever cases that was reconfirmed by the Municipal Health Services and that were included in the national case register decreased from $90 \%$ in 2009 , to $22 \%$ and $24 \%$ in 2010 and 2011, respectively. The decrease was observed in all categories of cases, including those considered to be confirmed by laboratory criteria. Continued use of a pre-outbreak case definition led to overreporting of cases to the Municipal Health Services in the post-epidemic years. Therefore we recommend dynamic laboratory notification rules, by reviewing case definitions periodically in an ongoing epidemic, as in the Dutch $Q$ fever outbreak. 


\section{Introduction}

$Q$ fever is a zoonotic disease and human infections result mainly from inhalation of Coxiella burnetii-contaminated aerosols [1-3]. Domestic ruminants are the main reservoir of the causative pathogen. Infected animals can shed C. burnetii in their milk or faecal excretions. Infections in humans can occur after contact with infected animals or contaminated dust [1-3]. Acute $Q$ fever is mostly self-limiting, but antibiotic treatment can reduce the duration of symptoms [1-3]. Early detection of $Q$ fever cases is hampered by the atypical polymorphic presentation of symptoms, ranging from asymptomatic to influenza-like illness, fever and pneumonia in acute infections. Therefore underreporting is quite substantial, especially in the beginning of an outbreak or if there is no knowledge of possible animal contact history [1-3]. The incubation period for $C$. burnetii infections is generally $9-40$ days. About $1-5 \%$ of all $Q$ fever cases may progress to chronic infection, often leading to a lifethreatening endocarditis or vascular infection [1, 2].

$Q$ fever has been a notifiable disease since 1976 in the Netherlands. The head of diagnostic microbiology laboratories and the treating physicians are obliged to notify the local public health authorities (the Municipal Health Services, MHS) of possible cases [4]. In accordance with the Public Health Act, the MHS provides anonymised reports to the national case register (NCR) of notifiable diseases [4]. The decision of the MHS to report acute $Q$ fever cases to the NCR is based on the combination of laboratory supporting evidence together with clinical information. Soon after a patient is notified to the MHS, clinical information is acquired by a MHS infectious disease specialist by consulting the physician and by questioning the patient using a questionnaire. Whenever a patient presents with at least one of three parameters (fever, pneumonia or hepatitis), a case is reconfirmed and reported to the NCR.

$Q$ fever diagnosis is based on DNA detection by polymerase chain reaction (PCR) and serology [5, 6]. The presence or absence of four different types of antibodies determines different stages of the infection. IgM and IgG antibodies against $C$. burnetii phase II antigen have been associated with early stages of illness, whereas IgM phase I and especially IgG phase I antibodies are indicative of ongoing (chronic) $Q$ fever [2].

Various serological tests are available for acute $Q$ fever, including indirect fluorescent antibody tests (IFATs), enzyme-linked immunosorbent assays 
(ELISAs) and complement fixation tests (CFTs). Recently, it was shown that the performances of these tests, in terms of confirming acute $Q$ fever, were comparable [7]. In our laboratory in the south-east of Brabant (Laboratory for Pathology and Medical Microbiology (PAMM), Veldhoven), all three serological tests listed, together with $\mathrm{PCR}$, are used for the diagnosis of acute $\mathrm{Q}$ fever. An algorithm was developed that supports the choice of PCR and/or serology based on the time between the first day of symptom onset and serum collection [8].

The south of the Netherlands experienced a large-scale outbreak of $Q$ fever over three consecutive years (2007-2009), with the highest peak in 2009 [9, 10]. At the beginning of the outbreak, a national case definition, including laboratory diagnostics, was established and used for mandatory notification [11]. This case definition was not modified during the outbreak, and was based on the knowledge available in the Netherlands about $Q$ fever diagnostics at that time, which was quite limited. As this outbreak constitutes one of the largest outbreaks ever recorded, with more than 4,000 acute Q fever cases notified, comparative laboratory-based studies yielded new insights on diagnostic markers for acute $Q$ fever. Most importantly, it was shown that the serological response after infection is long-lasting and that IgG phase II as well as IgM phase II antibodies are detected in more than half of acute $Q$ fever cases a year after symptom onset [7]. This implies that the serological diagnosis of acute $Q$ fever based on a single serum sample - which was part of the case definition - can be inaccurate.

In the study presented here, we evaluated the specificity of the laboratorydefined acute $Q$ fever cases during (2009) and after the epidemic (2010 and 2011). We also wanted to illustrate that interactions between the laboratory, physician and public health staff become more complex during an ongoing epidemic. Adaptation of the interpretation of laboratory results is warranted more and more in such a situation, especially when the laboratory techniques change over time.

\section{Materials and methods}

\section{Case definition}

The current Dutch case definition for acute $Q$ fever, based on clinical parameters and laboratory results, was used [11], i.e. any person who presents with at least one of three symptoms: fever, pneumonia and/or hepatitis, 
combined with positive laboratory results from any of the following three tests:

- detection of C. burnetii DNA in serum of patients without signs of chronic $Q$ fever;

- serum conversion or fourfold rise in IgG-specific phase II antibody titer by IFAT or CFT;

- $\quad$ single serum detection of IgM phase II antibodies by IFAT or ELISA with or without IgG phase II antibodies.

\section{Diagnostic tests and outcomes}

During the study period (January 2009 to December 2011), new tests were implemented in our laboratory, situated in the south-east of the province of Brabant. The evolution of the outbreak challenged the diagnostic capacities in the affected regions (figure 1). Firstly, ELISA IgM phase II was introduced, to improve throughput time by automation. Secondly, IFAT was introduced, to improve specificity. Finally, PCR was introduced, to improve early diagnosis (table 1). A detailed description of the different test methods has been published [9]. Due to the introduction of new techniques throughout the years, seven different outcomes lead to case notification to the MHS (table 2). On the basis of the laboratory criteria, whenever PCR was positive or a fourfold rise in IgG phase II antibodies was measured using CFT (outcomes 1 and 2, table 2), the patient was considered to be a confirmed acute $Q$ fever case. Whenever IgG phase II antibodies using CFT and IgM phase II antibodies using ELISA and/or IFAT were measured in a single serum sample, the patient was considered a probable case of acute $Q$ fever (outcomes 3, 4 and 5). When only IgM phase II antibodies using ELISA and IFAT were detected, cases were considered to be possible acute cases (outcomes 6 and 7). 


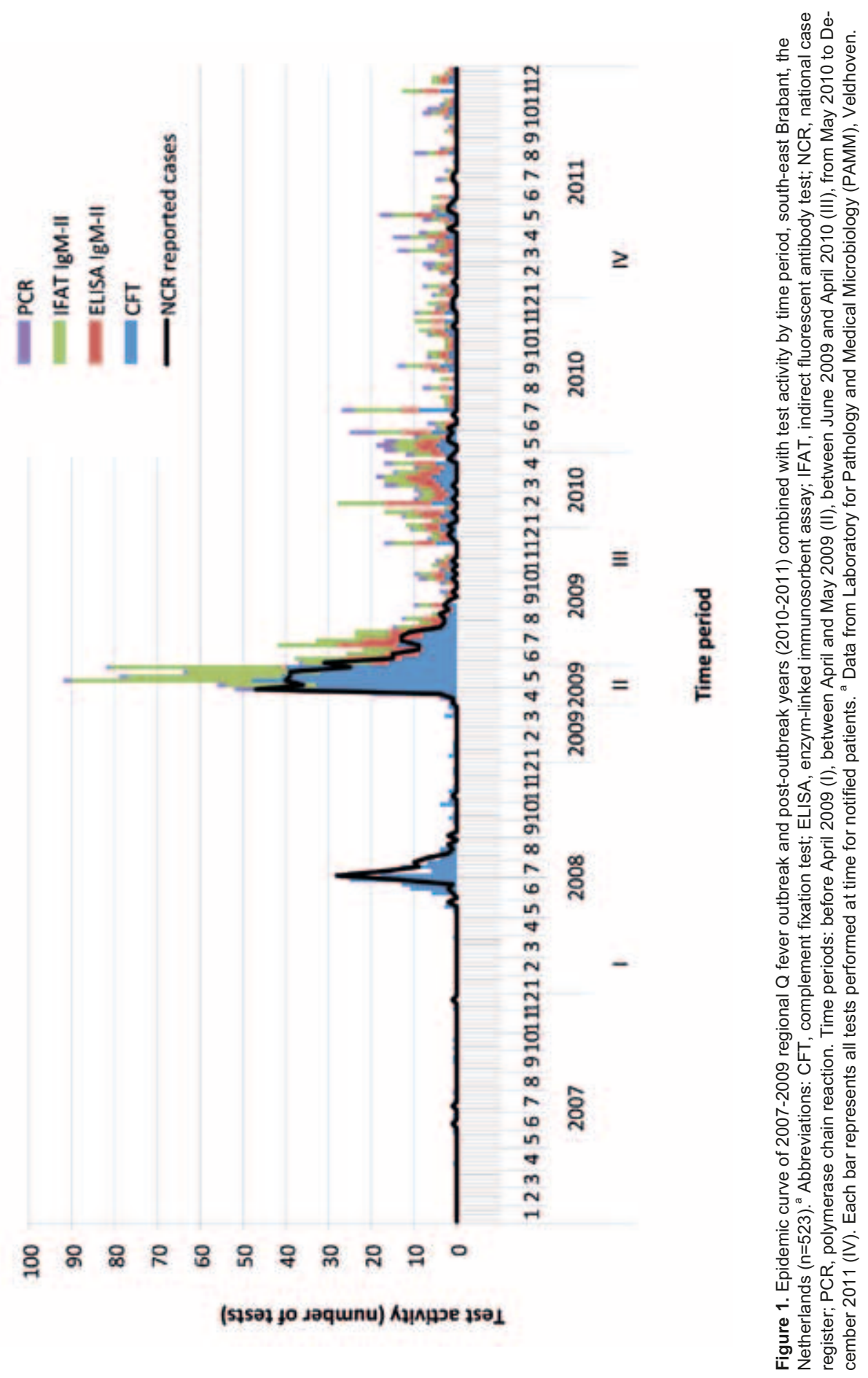


Table 1. Test methods used during (2009) and after (2010-2011) the 2007-2009 Q fever outbreak, the Netherlands.

\begin{tabular}{|c|c|c|}
\hline Time period & Duration of symptoms ${ }^{b}$ & Methods \\
\hline \multirow[t]{2}{*}{ I January - March 2009} & Not applicable & CFT \\
\hline & & $\mathrm{PCR}^{\mathrm{c}}$ \\
\hline \multirow[t]{3}{*}{ II April 2009 - May 2009} & Not applicable & IFAT IgM phase II \\
\hline & & CFT \\
\hline & & $\mathrm{PCR}^{\mathrm{c}}$ \\
\hline \multirow[t]{3}{*}{ III June 2009 - April 2010} & Not applicable & ELISA IgM phase II confirmed by IFAT IgM phase II $^{d}$ \\
\hline & & CFT \\
\hline & & $\mathrm{PCR}^{\mathrm{b}}$ \\
\hline \multirow[t]{3}{*}{ IV From April 2010} & $\geq 21$ days or unknown & ELISA IgM phase II confirmed by IFAT IgM phase $\|^{d}$ \\
\hline & & CFT \\
\hline & $<21$ days & $\mathrm{PCR}^{\mathrm{e}}$ \\
\hline
\end{tabular}

Abbreviations: CFT, complement fixation test; ELISA, enzym-linked immunosorbent assay; IFAT, indirect fluorescent antibody test; PCR, polymerase chain reaction.

${ }^{a}$ Data from Laboratory for Pathology and Medical Microbiology (PAMM), Veldhoven.

${ }^{b}$ An algorithm, based on the time between the first day of symptom onset and serum collection, comprises the use of PCR and serology tests. For patients sampled within the first two weeks of illness, it is recommended to perform PCR. For patients having first contact with a physician later than 2 weeks post-symptom onset or for patients for whom the date of symptom onset is not known, serology is recommended as the initial test.

${ }^{c}$ Before April 2010, PCR was occasionally performed in an external laboratory. Whenever PCR was negative IgM phase II testing was performed (ELISE IgM phase II confirmed by IFAT IgM phase II).

${ }^{d}$ ELISA IgM phase II positive samples were confirmed using IFAT.

${ }^{\text {e }}$ From April 2010, PCR was implemented in routine Q fever testing in the laboratory. Whenever PCR was negative, IgM phase II testing was performed (ELISA IgM phase II confirmed by IFAT IgM phase II).

Table 2. Laboratory-defined acute $Q$ fever patients based on test methods used, the Netherlands, 2007-2011.

\begin{tabular}{|c|c|c|c|c|c|c|}
\hline $\begin{array}{c}\text { PCR } \\
\text { positive }\end{array}$ & $\begin{array}{c}\text { CFT IgG } \\
\text { phase II } \\
\text { increase }^{a}\end{array}$ & $\begin{array}{l}\text { CFT IgG } \\
\text { phase II } \\
\text { positive }^{b}\end{array}$ & $\begin{array}{c}\text { ELISA IgM } \\
\text { phase II } \\
\text { positive }\end{array}$ & $\begin{array}{c}\text { IFAT IgM } \\
\text { phase II } \\
\text { positive }^{c}\end{array}$ & Outcome & Laboratory interpretation \\
\hline \multirow[t]{7}{*}{$x$} & & & & & 1 & Confirmed case \\
\hline & $x$ & & & & $2^{d}$ & Confirmed case \\
\hline & & $x$ & $x$ & $x$ & 3 & Probable case \\
\hline & & $x$ & & $x$ & 4 & Probable case \\
\hline & & $x$ & & & 5 & Probable case \\
\hline & & & $x$ & $x$ & 6 & Possible case \\
\hline & & & & $x$ & 7 & Possible case \\
\hline
\end{tabular}

Abbreviations: CFT, complement fixation test; ELISA, enzym-linked immunosorbent assay; IFAT, indirect fluorescent antibody test; PCR, polymerase chain reaction.

${ }^{\mathrm{a}}$ IgG phase II increase defined as a fourfold titer increase or seroconversion, measured by CFT.

${ }^{\mathrm{b}}$ Cut-off titer is $>1: 4$.

${ }^{\mathrm{c}}$ Cut-off titer is $\geq 1: 32$.

${ }^{d}$ A serum pair for one episode of $Q$ fever was defined whenever these sera were collected within 90 days. 


\section{Reporting to the national case register}

The notification system in the Netherlands works like a funnel: firstly, all laboratory-defined acute $Q$ fever patients (confirmed, probable and possible) were notified by the laboratory to the MHS. Secondly, the MHS infectious disease specialist reviewed all cases. If the case also met the clinical criteria, notification was reconfirmed and reported to the NCR. If the case did not meet these criteria, it was registered as not notifiable.

\section{Data analysis}

The dataset with the reconfirmed and not-notifiable acute $Q$ fever cases from MHS Brabant-South-East was merged with the laboratory test results, based on the patients' six-digit postal code and date of birth. After merging, retrospective analysis of the acute $Q$ fever cases during 1 January 2009 to 31 December 2011 was conducted. Descriptive analysis included age and sex. Cases were grouped according to the laboratory interpretations (1-7) and reviewed to assess what proportion was finally included in the NCR.

Statistical analysis included computations of laboratory-outcome frequencies per year and the percentage of reconfirmed notified and not-notifiable cases per year. Significance in these groups computed using one-sided MannWhitney test and Fisher's exact test, respectively.

\section{Results}

\section{Patients' characteristics}

From 1 January 2009 to 31 December 2011, 658 Q fever patients were reported by laboratories in south-east Brabant to the MHS, of which 523 were from our laboratory. The remaining 135 patients were reported by other laboratories in the region and were not included in the analysis. The mean age of the 523 patients was 49 years (standard deviation (SD): 16), and 320 $(61.2 \%)$ were male.

\section{Diagnostic tests and outcomes}

Of the 523 laboratory-defined cases notified to the MHS by our laboratory, 377 occurred in 2009, 96 in 2010 and 50 in 2011. During these three years, the distribution of laboratory outcomes changed substantially (table 3 ): in $2009,49.3 \%$ (186/377) of the patients in the MHS database were considered confirmed cases, mainly based on IgG seroconversion. Although PCR was 
indicated for most of the cases, it was seldom performed because this technique was not implemented in our laboratory before April 2010 . Only $17.2 \%$ (65/377) of the notified patients in 2009 were considered possible cases, based on single IgM phase II response. The distribution changed over the years, with far fewer patients belonging to the confirmed category based on laboratory criteria in 2010 and 2011. The category of patients considered to be least certain (possible), based on laboratory criteria, increased over time, constituting almost half of the patients diagnosed in 2011. These differences in distribution were significant when comparing 2010 vs 2011 and 2009 vs 2011 (table 3).

\section{Reporting to the national case register}

In 2009, all laboratory-confirmed cases, except two, were reconfirmed by MHS and reported to the NCR (184/186) (table 4). In 2010 and 2011, the proportion of reconfirmed cases dropped: only $7 / 12$ and 4/9 of the cases considered to be confirmed by laboratory criteria in 2010 and 2011 respectively were reported to the NCR. Interestingly, six of the 10 cases reported in 2010 and 2011 not reconfirmed were PCR positive in the initial serum sample, and therefore in laboratory terms would be considered true positives. Follow-up serology was performed in four of these six cases: IgM phase II as well as IgG phase II antibodies against $C$. burnetii were not detected. 


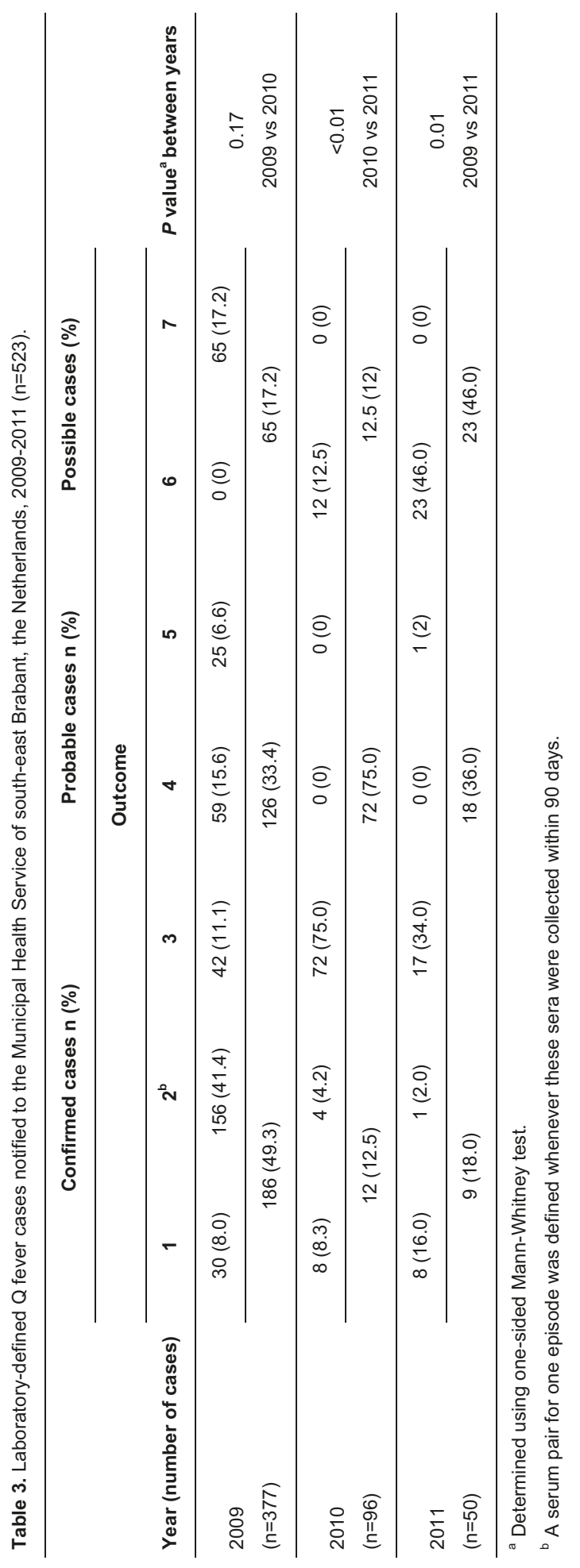




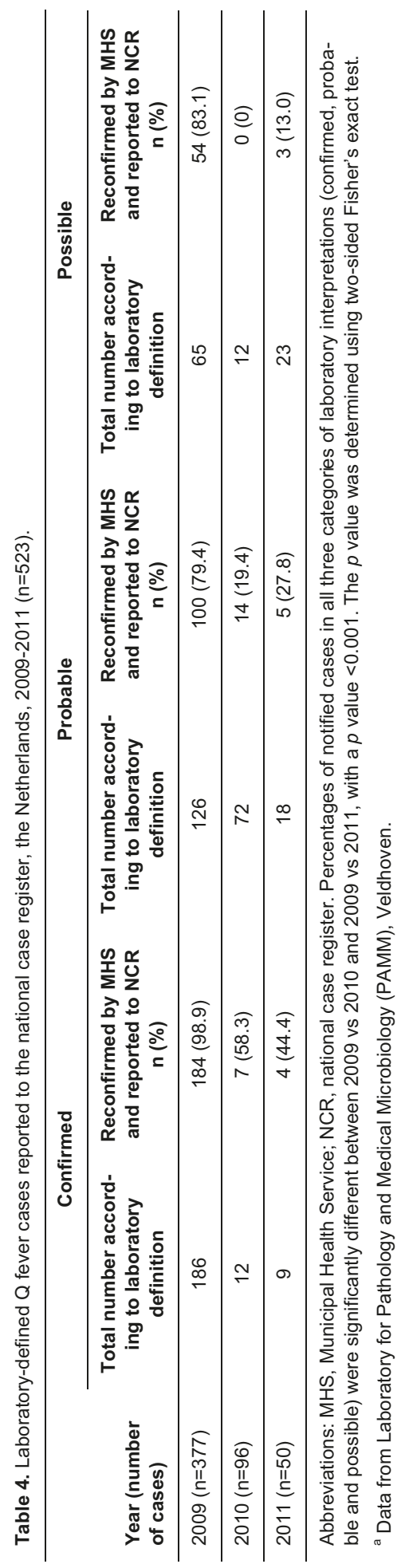




\section{Discussion}

In this study, we showed that the continued use of a pre-outbreak case definition lead to over-reporting of acute $Q$ fever to the MHS in the postepidemic years. This was caused by the increasing seroprevalence in the population and the observed persistence of IgM antibody titers, both influencing the interpretation of serological test results. Therefore, a test result that was diagnostic for acute disease at the start of the outbreak became almost useless later on. For instance, the specificity of single IgM phase II positive results, in terms of inclusion in the NCR, was $83.1 \%$ in 2009 , whereas in 2010 and 2011, the specificity dropped to $0 \%$ and $13 \%$ respectively. According to national law, all notifications of laboratories and physicians should be investigated by the MHS infectious disease specialist in order to track down (new) sources, the principal reason for notification of $Q$ fever. The over-reporting observed here in the course of a large outbreak does not aid such source finding.

Laboratory diagnosis of acute $Q$ fever is quite complex. Culture techniques for $C$. burnetii are not available for routine practice, and therefore diagnosis relies on pathogen detection by PCR or serology or both. Although detection of $C$. burnetii DNA in serum by PCR is highly sensitive, it is also timedependent, with a window for detection of approximately two weeks after onset of acute $Q$ fever symptoms [5]. Besides, not all diagnostic laboratories have $C$. burnetii PCR facilities. Therefore, serological tests will remain necessary in laboratory diagnosis of (acute) $Q$ fever. The variety of serological techniques and the implementation of new techniques throughout recent years make the diagnostic algorithms even more challenging. In our study period, 2009-2011, procedures changed three times: IFAT was introduced April 2009 for improvement of specificity, ELISA was implemented in June 2009 to increase the throughput time, and PCR was implemented in April 2010 in our laboratory to shorten the time to diagnosis (table 1 and figure) Such changes are unavoidable during a large-scale outbreak: routine methods that were in place at the start of the outbreak were not suitable for larg escale use. Changing diagnostic methods in during an outbreak may influence the case definition used. Closer interaction between laboratory diagnosticians, epidemiologists and MHS infectious disease specialists is highly 
recommended: it will help unravel the notifications and focus investigation on real acute $Q$ fever cases.

The current laboratory criteria for notification contain confirmed laboratory cases (PCR positive or a fourfold increase in IgG phase II titer) and probable and possible cases (single serum IgM phase II positive) [11]. The last criterion, applied to patients with specific clinical symptoms, had a high positive predictive value in the epidemic year (2009). After the epidemic was successfully managed at the end of 2009, the incidence of acute $Q$ fever fell drastically from 2010 onwards [12]. However, seroprevalence was high in the affected areas, reaching up to $20 \%$ of the population in some highly affected areas in the south of the Netherlands [13]. The number of laboratory requests remained high as the diagnostic triaging changed with increasing awareness. The total number of samples tested at our laboratory for acute $Q$ fever increased from 4,516 in 2009 to 5,138 in 2010. Two years after the epidemic, the numbers decreased to 3,116 in 2011 and 2,946 in 2012. The group of patients subjected to diagnostic testing widened, thereby changing the positive predictive value of the same test outcome. Also, in the years after the epidemic, the indication for diagnostic testing changed from acute illness to (chronic) fatigue symptoms. Microbiologists can signal such changes but only if clinical information and other relevant background information is provided by the physician requesting the tests. However, this information is often lacking. As a consequence, the laboratory kept reporting IgM phase II positive patients to the MHS. IgG phase II and IgM phase II remain detectable for a long time after initial infection. Recently, it was shown that at least until 12 months after acute $Q$ fever IgM phase II antibodies are detectable in the majority of the patients [7]. Since most of the acute $Q$ fever infections remain asymptomatic, IgM phase II in patients without classic symptoms represented mostly past infections instead of new acute cases in the post-epidemic period.

The need for more direct interaction between treating and notifying physicians and the laboratory was also illustrated by another finding: in our study, we found four PCR-positive cases (in 2010 and 2011) that were not included in the NCR. The reason for not reconfirming these cases was the aspecific clinical presentation together with the lack of serological response in followup sera from some of the cases, casting doubt on the performance of the diagnostic test. Positive PCR without serological response weeks after initial symptom onset is highly unlikely. Although it has been suggested that anti- 
body responses may be limited when antibiotic treatment is started early, this was not observed in a recent study [14]. Therefore, finding PCR-positive patients whose infection cannot be confirmed by serology suggests that they were due to false-positive PCR tests. PCR, which is highly sensitive, has always been prone to false-positive results, requiring strict protocols. Regretfully, despite these strict protocols, positives were still found that did not match the clinical picture, and again, with widening referral of patients, the positive predictive value of the same assay decreases. The combination of diagnostic serology and PCR can be problematic, as laboratory contamination may result in antigen preparations containing high levels of $C$. burnetii DNA [15]. After this phenomenon was reported, separate samples for serological and PCR tests were taken from patients, to reduce the potential of cross-contamination.

Although serological positive results do indicate infected patients, the inability to discriminate acute, chronic and past infections based on these criteria placed a lot of emphasis on the decision-making by the MHS. Notification of past infections does not support source finding, but will give MHS staff ne edless work. Therefore we recommend 'downsizing' the notification criteria, as was done in other infectious diseases [16, 17]. For example, at the start of the influenza $A(\mathrm{H} 1 \mathrm{~N} 1)$ pdm09 epidemic in 2009 and 2010, it was mandatory to report all new patients, but this was rapidly changed to hospitalized patients only. Notification was abandoned totally when influenza A(H1N1)pdm09 became seasonal influenza [17].

The timing of change in notification criteria is rather arbitrary, but based on our retrospective data we conclude that the criteria could have been modified in 2010 for the Q fever-endemic areas in the Netherlands. We would recommend changing the laboratory case definition to rely on more specific testing as the incidence decreases and seroprevalence increases in a previously affected region by omitting IgM phase II positive sera and only report laboratory-confirmed cases. Whenever this is based on PCR positive sera, serological confirmation is recommended to monitor false-positives due to contamination. Narrowing the notification rule to confirmed cases only is likely to lead to a more meaningful epicurve; notifications that are pending would no longer be taken into account. In order to keep a close eye on an epidemic, clinicians together with laboratories should closely follow up patients after a first positive result. 
This study emphasises the importance of updating diagnostic criteria during an outbreak due to emerging pathogens. New notification rules in the aftermath of an epidemic are therefore necessary. As knowledge increases and diagnostic technology improves, definitions need to be changed to reflect those trends. We suggest a case definition that includes a degree of certainty (e.g. probable or confirmed) based on the different type of laboratory results. 


\section{References}

1. Maurin M, Raoult D. Q fever. ClinMicrobiol Rev 1999;12(4):518-53.

2. Parker NR, Barralet JH,Bell AM. Q fever. Lancet 2006;367(9511):679-88.

3. Raoult $D$, Marrie $T$, Mege J. Natural history and pathophysiology of $Q$ fever. Lancet Infect Dis 2005; 5(4):219-26.

4. Wet publieke gezondheid. Wet van 9 oktober 2008, houdende bepalingen over de zorg voor de publieke gezondheid (Wet publieke gezondheid).[Public Health Act. Law of 9 October 2008, containing provisions on the care of public health (Public Health Act).2008.

Pub. L. No. BWBR0024705. Dutch. Available from: http://wetten.overheid.nl/BWBR0024705/geldigheidsdatum_26-09-2013

5. Schneeberger PM, Hermans MH, van Hannen EJ, Schellekens JJ, Leenders AC, Wever PC. Real-time PCR with serum samples is indispensable for early diagnosis of acute $Q$ fever. Clin Vaccine Immunol. 2010;17(2):286-90.

6. Fournier PE, Raoult D. Comparison of PCR and serology assays for early diagnosis of acute Q fever. J Clin Microbiol. 2003;41(11):5094-8.

7. Wegdam-Blans MC, Wielders CC, Meekelenkamp J, Korbeeck JM, Herremans T, Tjhie $\mathrm{HT}$, et al. Evaluation of commonly used serological tests for detection of Coxiellaburnetii antibodies in well-defined acute and follow-up sera. Clin Vaccine Immunol. 2012;19(7):1110-5.

8. Wegdam-Blans MC, Nabuurs-Franssen MN, Horrevorts AM, Peeters MF, Schneeberger PM, Bijlmer HA. [Laboratory diagnosis of acute $Q$ fever]. Ned TijdschrGeneeskd. 2010;154:A2388. Dutch.

9. Delsing CE, Kullberg BJ, Bleeker-Rovers CP. Q fever in the Netherlands from 2007 to 2010. Neth J Med. 2010;68(12):382-7.

10. Dijkstra F, van der Hoek W, Wijers N, Schimmer B, Rietveld A, Wijkmans CJ, et al. The 2007-2010 Q fever epidemic in the Netherlands: characteristics of notified acute Q fever patients and the association with dairy goat farming. FEMS Immunol Med Microbiol. 2012;64(1);3-12.

11. National Institute for Public Health and the Environment (RIVM). Q-koorts. [Q fever]. A78. LCl / Clb / RIVM directive infectious disease control . Bilthoven: RIVM; April 2010, last updated March 2011. Dutch. Available from: http://www.rivm.nl/dsresource?objectid=rivmp:6826\&type=org\&disposition=inline

12. van der Hoek W, Hogema BM, Dijkstra F, Rietveld A, Wijkmans CJ, Schneeberger PM, et al. Relation between $Q$ fever notifications and Coxiellaburentii infections during the 2009 outbreak in the Netherlands. Euro Surveill. 2012;17(3):pii=20058. Available from: http://www.eurosurveillance.org/ViewArticle.aspx?Articleld=20058

13. Kampschreur LM, Hagenaars JC, Wielders CC, Elsman P, Lestrade PJ, Koning OH, et al. Screening for Coxiellaburnetiiseroprevalence in chronic $Q$ fever high-risk groups reveals the magnitude of the Dutch Q fever outbreak. Epidemiol Infect 2013;141:84751.2012;13:1-5.

14. Wielders CC, Kampschreur LM, Schneeberger PM, Jager MM, Hoepelman AI, Leenderes $A C$, et al. Early diagnosis and treatment of patients with symptomatic acute $Q$ Fever do not prohibit IgG antibody responses to Coxiellaburnetii. Clin Vaccine Immunol. 2012;19(10):1661-6. 
15. Tilburg JJ, Horrevorts AM, Peeters MF, Klaassen CH, Rossen JW. Identification by genotyping of a commercial antigen preparation as the source of a laboratory contamination with Coxiellaburnetii and as an unexpected rich source of control DNA. J ClinMicrobiol. 2011;49(1):383-4.

16. van 't Klooster TM, Wielders CC, Donker T, Isken L, Meijer A, van den Wijngaard CC, et al. Surveillance of hospitalisations for 2009 pandemic influenza $A(H 1 N 1)$ in the Netherlands, 5 June - 31 December 2009. Euro Surveill 2010;15(2):pii=19461. Available from: http://www.eurosurveillance.org/ViewArticle.aspx?Articleld=19461

17. Prince HE, Tobler LH, Yeh C, Gefter N, Custer B, Busch MP. Persistence of West Nile virus-specific antibodies in viremic blood donors. Clin Vaccine Immunol. 2007;14(9):122830. 



\section{Chapter 4}

\section{Chronic $Q$ fever: review of the literature and a proposal of new diagnostic criteria}

Journal of infection 2012; 64(3): 247-59

M.C.A. Wegdam-Blans*

L.M. Kampschreur*

C.E. Delsing

C.P. Bleeker-Rovers

T. Sprong

M.E.E. van Kasteren

D.W. Notermans

N.H.M. Renders

H.A. Bijlmer

P.J. Lestrade

M.P.G. Koopmans

M.H. Nabuurs-Franssen

J.J. Oosterheert

and the Dutch $Q$ fever Consensus Group**

${ }^{*}$ both authors contributed equally 


\section{Abstract}

A review was performed to determine clinical aspects and diagnostic tools for chronic $Q$ fever. We present a Dutch guideline based on literature and clinical experience with chronic $Q$ fever patients in the Netherlands so far. In this guideline diagnosis is categorized as proven, possible or probable chronic infection based on serology, PCR, clinical symptoms, risk factors and diagnostic imaging. 


\section{Introduction}

$\mathrm{Q}$ fever is a zoonosis, caused by the Gram-negative coccobacillus Coxiella burnetii ( $C$. burnetii). There is a large animal reservoir, with goats, sheep and cattle being the most common source of human infections [1-3]. After primary infection, an estimated $1-5 \%$ of patients progress to chronic $Q$ fever, which can become manifest years after initial infection. Endocarditis and infections of aneurysms or vascular prostheses are the most common manifestations [4, 5]. Arthritis, osteomyelitis or hepatitis are rare manifestations of chronic $Q$ fever. In a small number of patients no focus can be found [6-8]. Pre-existent cardiac valvular disease, aortic aneurysm, vascular grafts, immunocompromised state, and pregnancy are reported risk factors for the development of chronic $Q$ fever [7, 9, 10].

If left untreated, chronic $Q$ fever leads to considerable morbidity and a mortality up to $60 \%$ [5]. Long-term antibiotic treatment, preferably consisting of hydroxychloroquine and doxycycline, and sometimes aggressive surgery are required in patients with established chronic $Q$ fever $[4,5,11]$. The consequences of an adequate diagnosis are therefore of major impact for suspected chronic $Q$ fever patients. However, the diagnosis of chronic $Q$ fever is challenging: culture of $C$. burnetii is difficult and time-consuming, requires a level 3 biosafety laboratory and lacks sensitivity [12]. The diagnosis of chronic $Q$ fever currently relies on serology and detection of DNA in blood or tissue with polymerase chain reaction (PCR). Chronic $Q$ fever is proven when $C$. burnetii DNA is detected by PCR in blood or tissue in the absence of acute infection, although sensitivity of these techniques is low [13]. Serological diagnosis is based on antigenic variation of $C$. burnetii, in which after culture in cells, a virulent variant phase I antigen, shifts to an avirulent phase II variant. During acute infection, antibodies to phase II antigens are detected first, followed by phase I antibodies. Persisting high levels of antibodies to phase I and phase II antigens, are thought to be caused by continuous antigenic stimulation and considered indicative for chronic $Q$ fever $[2,14]$. A cutoff for phase I IgG of $\geq$ 1:800 has been internationally accepted for the serological diagnosis of chronic $Q$ fever, which is based on an in house produced immunofluorescence assay (IFA) [15]. Due to a high number of false-positive tests with a cut-off of 1:800 and development of other (commercial) IFA-assays, discussions about 
the optimal cut-off values and suggestions of the incorporation of clinical characteristics in the diagnosis of chronic $Q$ fever have emerged [8, 16, 17].

Since 2007, the southern parts of the Netherlands suffered from a major $Q$ fever outbreak, with more than 4000 reported symptomatic cases. As the majority of patients have mild or asymptomatic acute infection, the actual incidence is probably much higher [18]. Due to hygienic measures, vaccination of goats and culling of pregnant goats, the acute $Q$ fever epidemic in the Netherlands has subsided. Nevertheless, chronic $Q$ fever is seen in a rising number of patients [19]. Uniformity in the assays and interpretation of test results is necessary for good patient care. Given the uncertainties in diagnostic algorithms for chronic $Q$ fever, the impact of an adequate diagnosis on the prognosis and treatment, and the increasing number of patients, we performed a review of the available literature on the clinical aspects and diagnostic tools for chronic $Q$ fever. Based on this available evidence, a new proposal for the diagnosis of chronic $Q$ fever was formulated in a consensus meeting of clinicians and microbiologists which combines clinical, radiological and microbiological factors.

\section{Materials and Methods}

\section{Search strategy}

A literature search was conducted in Pubmed to identify publications on the diagnostic features of chronic $Q$ fever. We used the MESH-terms " $Q$ fever" and "Coxiella burnetii" in combination with the subheading "diagnosis" and "microbiology", and the MESH terms "radiography" OR "ultrasonography" OR "echocardiography" OR "Positron-Emission Tomography" OR "Immunology" OR "PCR" OR "fluorescent antibody technique" OR "serologic test" OR "diagnostic imaging". Language was restricted to "English" only. The reference lists of the identified publications were screened for relevant additional publications. No restrictions were set for the clinical reference standard method applied. Publications were eligible for inclusion if: (i) clinical parameters were described in well defined cohorts of chronic $Q$ fever patients or (ii) diagnostic tools for chronic $Q$ fever such as IFA, PCR, immunohistochemistry, or culture, were evaluated against the golden standard used at the time of publication. Case-reports and case series with less than five patients and reviews were excluded. Papers concerning other topics than human chronic $Q$ fever were excluded, such as studies about acute $Q$ fever and animal studies. Features 
solely associated with the localisation and complications of chronic $Q$ fever infection (for example heart failure and embolism in case of endocarditis, abdominal pain in case of infected aortic abdominal aneurysm) were not reviewed. If studies described the same patient cohorts, we only included the study which used the final and complete patient data.

\section{Data extraction}

Titles and abstracts were screened by two reviewers (MW and LK). If the title or abstract suggested a publication meeting the inclusion criteria, full text was obtained. Selected full text articles were read by both reviewers and included in agreement. All included publications were critically reviewed and summarized. The diagnostic and clinical data were extracted from each included publication and drawbacks and biases were noted. Microbiological, clinical, laboratory and radiological features were considered separately.

\section{Results}

\section{Selection}

We initially identified 607 unique publications describing features of chronic $Q$ fever. Based on title and abstract, 553 articles were excluded because they did not describe diagnostic accuracy or clinical manifestations. An additional eight publications were detected through the reference lists. After reviewing the 62 remaining papers, 45 articles were excluded because they were case reports or series describing less than five patients (16), they described acute $\mathrm{Q}$ fever only (6), pathogenesis only (2), epidemiology only (8), diagnostic microbiological parameters not suitable in clinical practice (4), tests validation without a clinical diagnosis (4), full text was not available in accessible libraries (2) or because the report presented a cohort of patients that was also included in a subsequent study (3). The remaining 17 articles met the inclusion criteria. Seven manuscripts described the microbiological diagnosis and 10 manuscripts described clinical symptoms and features. Of the latter, 8 articles described imaging techniques and 9 described laboratory parameters in patients with chronic $Q$ fever (figure 1).

\section{Clinical features of patients diagnosed with chronic $Q$ fever}

We found 10 studies describing clinical features of at least five chronic $Q$ fever patients $[4,5,7,10,20-25]$. Their results are summarized in table 1 . Table 2 
includes comments on these studies and descriptions of the definition of chronic $Q$ fever used. One article dealt with vascular chronic $Q$ fever only [4]. One article described endocarditis, vascular infections, as well as more rare manifestations of chronic $Q$ fever [7]. The remaining eight studies presented cases with $Q$ fever endocarditis only [5, 10, 20-25]. In case of $Q$ fever endocarditis, a diagnosis based on the modified Duke criteria, including phase I IgG $\geq 1: 800$, was most frequently used. Vascular chronic $Q$ fever was diagnosed when phase I IgG $\geq 1: 800$ was present in combination with a clinical aneurysm or vascular infection, or positive $C$. burnetii PCR or culture of vascular tissue. Fever was the most common presenting symptom of chronic $Q$ fever in $23-100 \%$ of the cases (data available in nine reports), followed by weight loss (data in eight reports), which was present in $11-100 \%$ of patients. Hepato- and/or splenomegaly were described in five reports, and were present in $11-90 \%$ of cases. Five reports mentioned fatigue in $9-100 \%$ cases. In three studies, night sweats were reported as presenting symptom in $26-100 \%$ of cases. One study also evaluated the absence of symptoms, which was observed in $6 \%$ of patients.

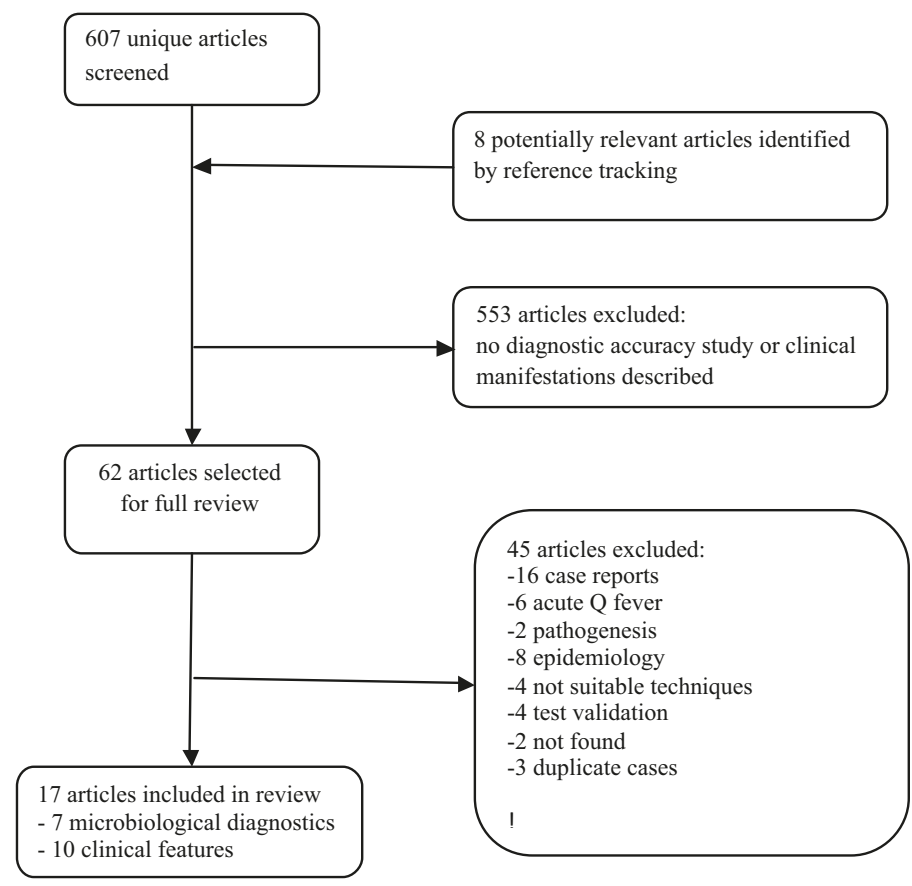

Figure 1. Flow-diagram of selection and revision of articles. 


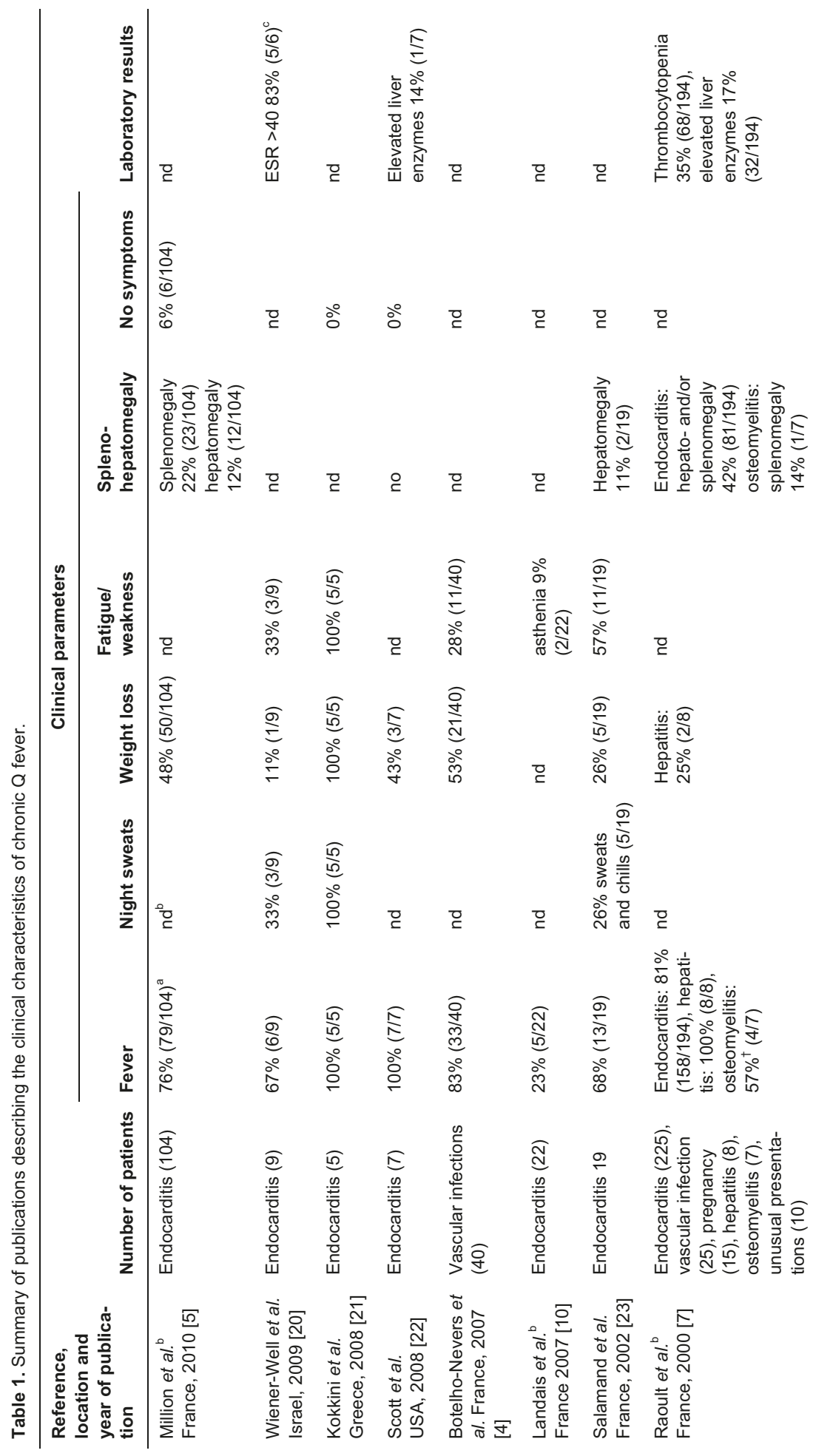




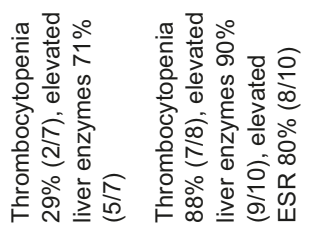

마 무

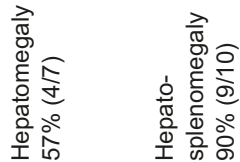

마 후

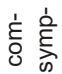

ते

무

$\frac{0}{\stackrel{0}{0}}$

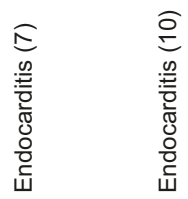

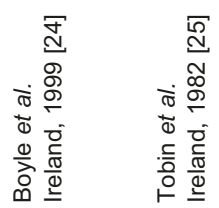

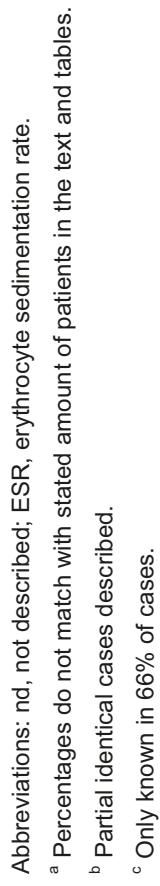


Table 2. Definitions of chronic $Q$ fever and comments on studies in table 1, 3 and 4.

\begin{tabular}{ll}
\hline & Definitions of chronic $\mathbf{Q}$ fever and comments on studies \\
\hline Frankel et al. & $\begin{array}{l}\text { Definition of chronic } Q \text { fever is object of study. Patients with positive C. burnetii serology } \\
\text { and echocardiographic imaging, which was classified according to the modified Duke } \\
\text { criteria in no, possible or definite endocarditis were compared. }\end{array}$
\end{tabular}

Million et al. $\quad Q$ fever endocarditis diagnosed (1) with modified Duke criteria which includes IFA, phase

France, 2010 [5] I lgG $\geq 1: 800$ and positive C. burnetii blood culture, and echocardiographic imaging, or (2) positive PCR on blood, serum, or valve samples. Unknown amount of patients with positive PCR or culture. Valvular insufficiency was not described according to revised Duke-criteria. Prosthetic valves not separately described.

Wiener-Well et al. Q fever endocarditis diagnosed with modified Duke criteria which include IFA, phase I Israel, 2009 [20] $\quad \operatorname{lgG} \geq 1: 800$ and positive $C$. burnetii blood culture, and transesophageal echocardiographic and/or histopathological findings. No confirmation of diagnosis with culture and/or PCR. ESR only in $66 \%$ of patients available.

Kokkini et al. Q Q fever endocarditis diagnosed with serology (type and cut-off not defined, but IFA phase Greece, 2008 [21] I lgG in all cases >1:1024) and echocardiographic imaging. No confirmation with culture and/or PCR.

Scott et al. USA, Q fever endocarditis diagnosed with IFA and CBR (cut-off not defined, but IFA phase I 2008 [22] Ig all >1:1024) and echocardiographic imaging according to modified Duke criteria. No confirmation with culture and/or PCR.

Botelho-Nevers et Vascular chronic $Q$ fever infection diagnosed by (1) positive culture or PCR in vascular al. France, 2007 biopsy or (2) IFA phase I IgG $\geq 1$ : 800, or positive blood PCR and a clinical suspicion of [4] aortic aneurysm or graft infection. Diagnosis chronic $Q$ fever only confirmed in 12/40 patients with positive $C$. burnetii culture or PCR on blood or tissue.

Landais et al. $\quad Q$ fever endocarditis diagnosed with modified Duke criteria which include IFA, phase I France, 2007 [10] IgG $\geq 1: 800$, and echocardiographic imaging. No confirmation with culture and/or PCR.

Fenollar et al. $\quad Q$ fever endocarditis diagnosed (1) with modified Duke criteria which includes IFA, phase France, 2004 [8] I IgG $\geq 1: 800$ and positive and echocardiographic imaging. Vascular infections were diagnosed on the basis of the presence of a vascular aneurysm or prosthesis associated with vascular symptoms, fever or phase I IgG $\geq 1: 800$ and no evidence of other infections

Rolain et al. $\quad Q$ fever endocarditis diagnosed with endocarditis according to the modified Duke criteria France, 2003 [26] and positive PCR or culture for C. burnetii from valve samples.

Lepidi et al. IFA IgG phase I $\geq 1: 800$. Control-group exists of culture positive endocarditis (non-Q France, 2003 [27] fever, defined by negative IFA); no PCR and culture for Q fever of this group.

Salamand et al. Q fever endocarditis diagnosed with modified Duke criteria which include IFA, phase I France, 2002 [23] IgG $\geq 1: 800$, and echocardiographic imaging. No confirmation with culture and/or PCR, two cases confirmed with histology of the removed valve.

Raoult et al. $Q$ fever endocarditis diagnosed with IFA, phase I IgG $\geq 1: 800$, and clinical findings, France, 2000 [7] defined in all subgroups as follows: endocarditis, modified Duke criteria; vascular infection, vascular aneurysm and prosthesis, osteoarticular infection, bone lesion; hepatitis prolonged disease with liver transaminase increase; pregnancy, all pregnant women and babys with antibodies (also <1:800); other, long evolution and no other etiologies. No or unknown confirmation with culture and/or PCR. Valvular insufficiency not described according to Duke-criteria. No description of location or clinical characteristics of vascular infections. Definition of pregnancy related chronic $Q$ fever unclear, therefore not to determine which amount of pregnant patients only had an acute infection during pregnancy.

Boyle et al.

$Q$ fever endocarditis diagnosed with $C F T$, phase I IgG $\geq 1: 200$. No confirmation with Ireland, 1999 [24] culture and/or PCR. 1 patient with phase I lgG $<1: 200$. Echocardiographic imaging only performed in 4 patients. 


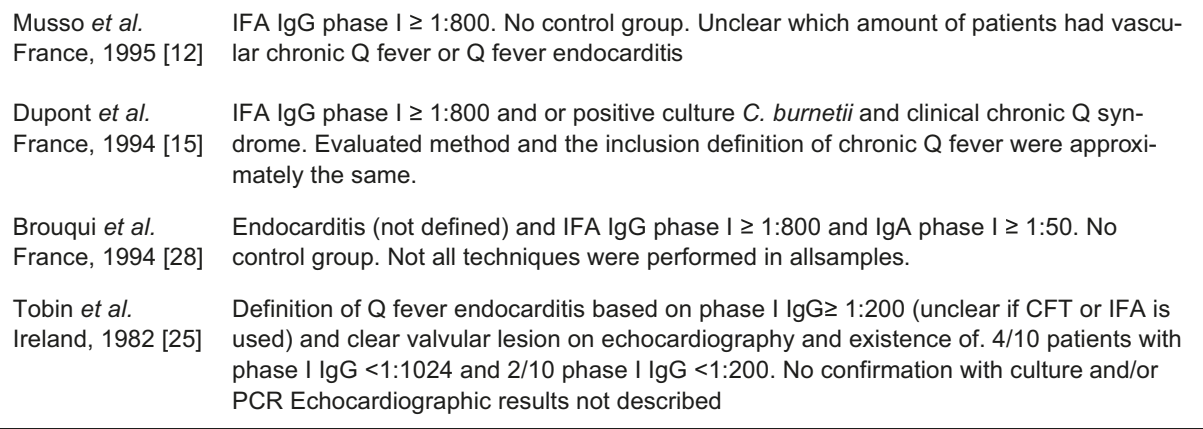

Abbreviations: IFA, Immunofluorescence assay; PCR, polymerase chain reaction; ESR, erythrocyte sedimentation rate; $\mathrm{CFT}$, complement fixation test.

\section{Biochemical laboratory features of patients presenting with chronic $\mathbf{Q}$ fever}

Biochemical laboratory features were scarcely described (table 1). Elevated liver enzymes were reported in four articles and present in $14-90 \%$ of cases, although exact quantification of the elevation was lacking [7, 22, 24, 25]. Thrombocytopenia was mentioned in three studies and present in $29-88 \%$ of cases [7, 24, 25]. Elevated erythrocyte sedimentation rate (ESR) was reported in two studies and present in $80-83 \%$ of cases [20, 25]. Table 2 includes comments on these studies and descriptions of the definition of chronic $Q$ fever used.

\section{Microbiological diagnosis of patients with chronic $Q$ fever}

We found seven publications that evaluated microbiological techniques for the diagnosis of chronic $Q$ fever (table 3) [8, 12, 13, 15, 26-28]. The reference standards for the definition of chronic $Q$ fever in these publications are summarized in table 2. Five of the reviewed studies included patients with $Q$ fever endocarditis only. In one study, both patients with $Q$ fever endocarditis and patients with vascular infection caused by $Q$ fever were included [13]. In one study, no clinical specifications were mentioned [15]. Five studies included a control group. Composition of the control group varied from patients with bacterial blood culture positive endocarditis without serological evidence of chronic $Q$ fever, blood donors, acute $Q$ fever disease, or serologically diagnosed non-endocardtitis related viral infection or a combination of these patients. In two studies, the control group was not specified. Three studies described sensitivity, specificity, positive predictive value (PPV) and negative predictive value (NPV) of different IgG phase I titers in chronic $Q$ fever patients $[8,15,26]$. 
Although studies of Dupont and Rolain are not completely comparable due to the different chronic $Q$ fever definitions used; sensitivity, specificity, positive predictive and negative predictive values were within the same range [15, 26]. Markedly different were the positive predictive values of Frankels' study. It was however not possible to collect other statistical characteristics from the reported data [8].

Four studies described the sensitivity of $C$. burnetii culture and detection of $C$. burnetii DNA by PCR in valve tissue and blood. Both culture and PCR were more sensitive, when performed on tissue compared to blood. Sensitivity of $C$. burnetii culture from cardiac valves was $88.9 \%(8 / 9)$ and $73.3 \%(11 / 15)$ in two studies $[27,28]$. Sensitivity of $C$. burnetii culture in blood specimens was only $40.0 \%(6 / 15)$ and $52.9 \%(9 / 17)[12,28]$. Comparable differences were found for DNA detection by PCR in blood and tissue with a sensitivity of $22.9 \%$ in blood specimens and $100 \%$ using cardiac valves [8, 27]. Reported specificity was $100 \%$. Sensitivity of immunohistochemical staining using mouse anti-C. burnetii monoclonal antibody on valves from patients was $55.5 \%(5 / 9)$ and $66.7 \%(10 / 15)[27,28]$. 


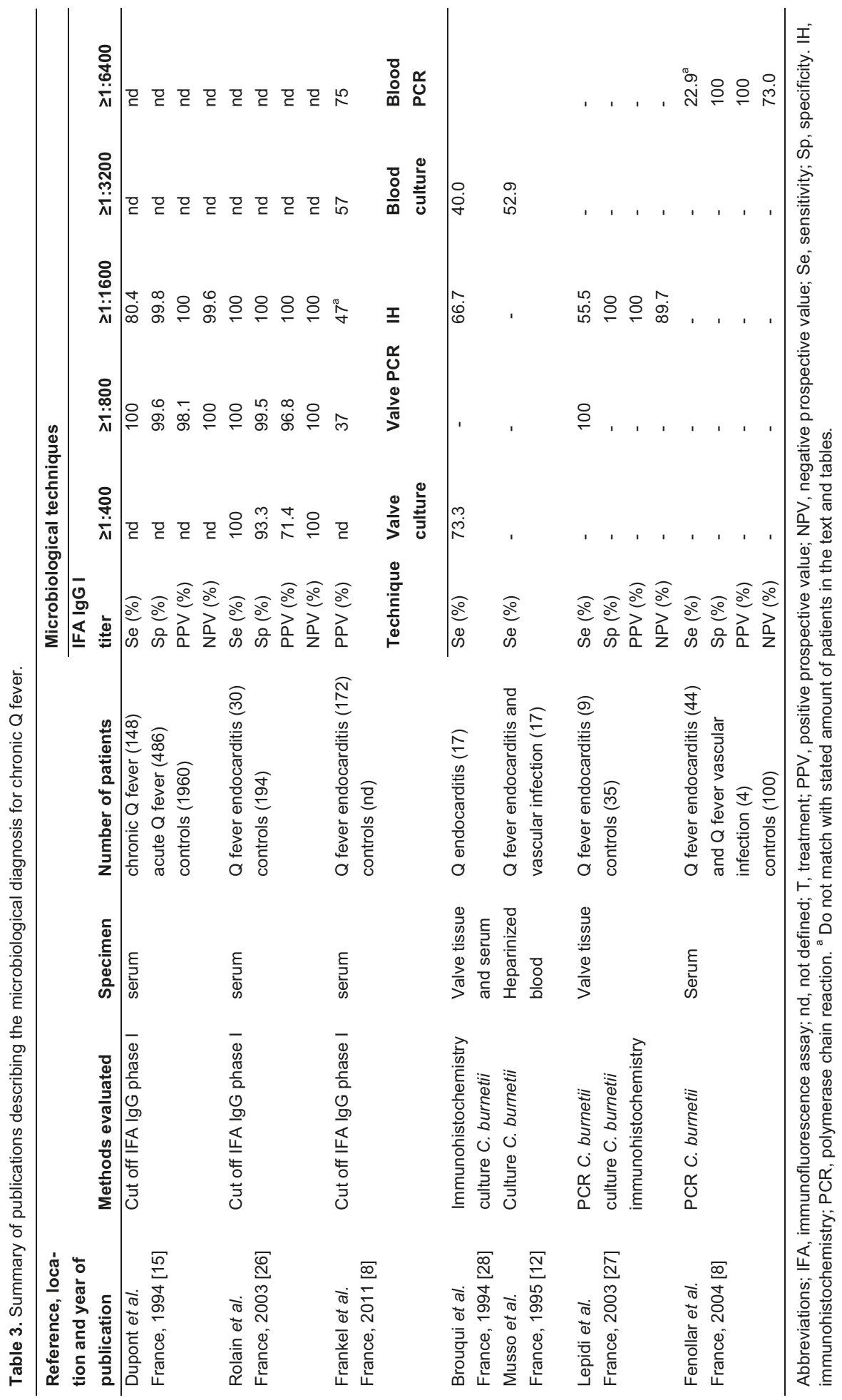




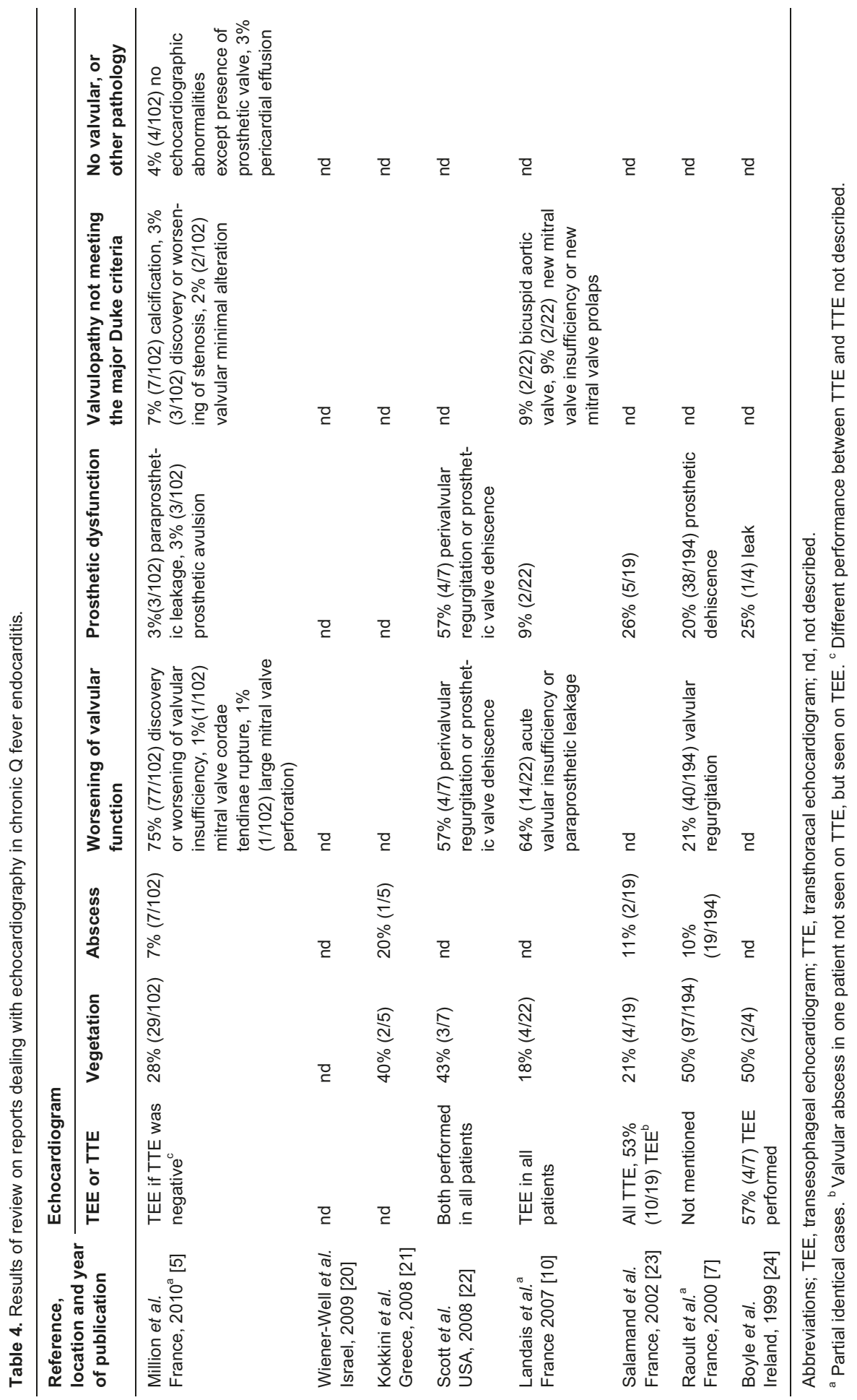




\section{Results of imaging techniques of patients diagnosed with chronic $Q$ fever}

Eight reports described results of echocardiographic imaging in patients diagnosed with $Q$ fever endocarditis (table 4) [5, 7, 10, 20-24]. Definitions of chronic $Q$ fever in these articles are described in table 2. Reports dealing with other imaging techniques, or imaging in non-endocarditis chronic $Q$ fever were not found. In the reviewed articles, the used echocardiographic technique was either not described, or it was unclear what percentage of patients underwent transthoracic echocardiography (TTE) and what percentage transesophageal echocardiography (TEE). Echocardiographic findings lacked description according to the content of the revised Duke criteria in most reports [29]. All major Duke-criteria were described in only two reports. Six reports described vegetations on echocardiography, which were present in $21-50 \%$ of cases. Prosthetic dysfunction was described in six reports (3$57 \%$ of cases). Worsening of valvular insufficiency was reported in 3 reports (21-75\% of cases). Cardiac (valve) abscesses were described in 4 reports (7-21\% of cases). In only 2 reports, valvulopathies not meeting the major Duke criteria, like calcifications, prolaps and stenosis were mentioned, although the exact cumulative percentage of these valvulopathies were not separately described. In addition, one manuscript reported that $4 \%$ of chronic $Q$ fever endocarditis patients had a prosthetic valve without echocardiographic abnormalities and found an incidence of pericardial effusion in $3 \%$ of chronic $Q$ fever endocarditis.

\section{Discussion}

With this review, we aimed to summarize the available knowledge on clinical presentation, microbiological diagnosis, and imaging techniques for the diagnosis of chronic $Q$ fever and to provide a basis for uniformity in the workup of patients suspected of having this infection. Due to the relative rarity of the disease, studies that have evaluated the diagnostic value of clinical, laboratory, imaging and microbiological parameters for chronic $Q$ fever are limited to case reports and are mainly retrospective, descriptive analyses of a limited number of patients. As there were no randomized trials available, meta-analysis and/or systematic review of the selected articles was not possible. Instead, we described the major outcomes of these studies. When 
evaluating the available evidence, the microbiological diagnosis of chronic $Q$ fever mainly relies on serology. In older papers differences in serological profiles between patients with acute, past and chronic infections were already notified, using ELISA, CFT as well as IFA: IgG phase I and to a lesser extent IgA phase I were markedly elevated in patients with chronic $Q$ fever [14, 30-34]. In some studies, chronic $Q$ fever used to be based on phase I $\lg G \geq 1: 200$ defined by complement fixation test (CFT). However, clinical validation of the CFT cut off was missing and its use in case of chronic $Q$ fever has been abandoned since. Also, the value of IgA antibodies in the diagnostics of chronic $Q$ fever has become less prominent. New microbiological tests and techniques for the diagnosis of (chronic) $Q$ fever are in development, but still need clinical evaluation and incorporation in clinical practice [35-38]. Dupont et al. were the first in 1994 to propose an IFA IgG phase I cut off $\geq 1: 800$ based on a retrospective serological study of 53 patients with chronic infections [15]. Drawback of this study is that the evaluated method and the definition of chronic $Q$ fever were approximately the same. Nevertheless, the Duke criteria for the diagnosis of endocarditis were modified in 2000 , including the proposed IFA IgG phase I titer $\geq 1: 800$ as a major criterion [29]. Subsequently, there have been publications in which the limitations of this cut-off value were described [17, 39-41]. High phase I IgG antibodies, for example, found in patients with cardiovascular risk factors, but without clinical symptoms, may pose a therapeutic dilemma. After all, when a diagnosis of chronic $Q$ fever is made, prolonged and intensive antibiotic therapy with a minimum of 18 months is warranted. Recently, a cut-off value of $1: 1600$ is suggested, based on re-evaluation of serological data and a high amount of false-positive tests with a cut-off of 1:800 [8]. The effect on the negative predictive value of a cut-off titer if 1:1600 was not mentioned. Conversely, chronic $Q$ fever cannot be excluded when phase I IgG antibody titer $<1: 800$ is detected [42]. Furthermore, inter and intra laboratory variations of immunofluorescence methods and results are probably of significant importance. Recently, Healy et al. showed that the concordance in result interpretation from three different centres was only 35\% [16]. Interpretation of serological results in the light of clinical information was therefore advised, although definition of these parameters were lacking in Healy's article. In the Netherlands most laboratories use the commercial IFA of Focus Diagnostics, with titration based on a multiple of 16 . An IgG phase I titer $\geq 1: 1024$ is cur- 
rently used as a cut-off value where above chronic $Q$ fever is conceivable [40].

Although collected from small patient cohorts, review of the test characteristics of culture and nucleic acid amplification techniques showed that sensitivity of C. burnetii DNA detection by PCR is markedly dependent on the type of specimen sampled. Sensitivity ranges from almost $100 \%$, when performed on (valve) tissue, to $47 \%$ and $23 \%$ respectively when culture and PCR are performed on peripheral blood. It is not known why yields are low when detecting C. burnetii in blood from chronic $Q$ fever patients, but as $C$. burnetii is an intracellular micro-organism it is conceivable that the cellular compartment (buffy coat) of peripheral blood is more sensitive for DNA detection. Fenollar et al. also hypothesized that circulating antibodies and immune complexes act as DNA scavengers [13].

Clinical features of patients with chronic $Q$ fever are only scarcely described in cohorts of five patients or more. Most important presenting features are non-specific findings such as fatigue, fever, weight loss, night sweats and hepato-splenomegaly, although the prevalence of all of these symptoms showed great variation between the different study cohorts. For example, one report described fever in $23 \%$ of cases, while another report mentioned fever in $100 \%$ of cases. One study found that $6 \%$ of chronic $Q$ fever cases remained without symptoms. Fatigue as a presenting symptom is often described, although in daily practice this is not a useful parameter, considering the overlap with $Q$ fever related fatigue syndrome which occurs in approximately $20 \%$ of patients after acute $Q$ fever infection $[19,43]$. Interpretation of the diagnostic value of the presenting symptoms currently described is hampered by the wide range in definitions of chronic $Q$ fever. In most definitions, diagnosis of chronic $Q$ fever was based on serological techniques; confirmation with PCR or culture was often not possible or the percentage of patients with a confirmed chronic $Q$ fever infection was not reported. It is interesting to define if different genotypes of $C$. burnetii are responsible for variation in clinical presentation and serological results. However, in the reviewed literature, these data were not provided.

Internationally accepted diagnostic criteria for infectious endocarditis are the revised Duke criteria in which echocardiography plays an important role [29]. Transesophageal imaging is superior to transthoracic echocardiography in detecting vegetations, particularly in patients with prosthetic valves [29]. However, in our reviewed reports, the used echocardiographic technique 
was commonly unclear or not described. Superiority of TEE was only described in one report in which a valvular abscess in one patient was not seen on TTE, but seen on TEE [23]. Our review shows that large pathologic findings demonstrating infective endocarditis (IE) are only found in a quarter of chronic $Q$ fever patients. Valvular insufficiency was the most common found feature on echocardiography in case of chronic $Q$ fever endocarditis, although strict definition and quantification of this finding were not reported. In a large proportion of patients with $Q$ fever endocarditis, echocardiographic findings are non-specific or even absent. These minor echocardiographic criteria, not being part of the revised Duke criteria, were only rarely confirmed by histology, C. burnetii culture or PCR in the available literature. Non-specific findings on pathologic examination of cardiac valves infected with $C$. burnetii have been described, although in those reports, comparison with echocardiographic results were missing $[5,28]$. Quantification of echocardiographic findings in case of chronic $Q$ fever endocarditis is not possible from our data. For that purpose, a systematic descriptive analysis of echocardiographic findings in patients with chronic $Q$ fever is obligatory.

For vascular $Q$ fever infections, no well described cohorts of patients that underwent radiological imaging are available. The only information is available from case reports [42, 44, 45]. Historically, computed tomography (CT) has been the standard for diagnostic imaging of vascular (graft) infections. MRI and duplex ultrasound are other options. However the positive predictive value of these diagnostic instruments is not optimal [46]. On CT scans, air bubbles can be detected around an infected graft in about half of the cases but are highly unspecific as in $50 \%$ of grafts these bubbles are present for weeks or even months after surgery. Moreover, it is difficult to differentiate between infection, hematoma, and lymphocele on CT scans. In chronic low-grade infections, CT is often false-negative [47]. In comparison to $\mathrm{CT}$, fluorodeoxyglucose positron emission tomography combined with $\mathrm{CT}$ (FDG-PET/CT) has shown a superior diagnostic performance in (low-grade) vascular infections. PET has shown a sensitivity exceeding $90 \%$ in comparison to $64 \%$ for CT [48]. Specificity exceeds $90 \%$ with the adoption of clear criteria for image interpretation (only focal, homogeneous, abnormal uptake interpreted as positive for infection) [46-49]. In order to avoid false-positive results, FDG-PET/CT should be performed at least 2 months after vascular surgery $[46,48,50]$. However, the role of PET/CT in detection of infected grafts or aneurysms by $C$. burnetii, which gives a low grade infection, has not 
been studied systematically yet. Two case reports in which PET/CT demonstrated superior diagnostic imaging compared to CT-scan in C. burnetii vascular graft and aneurysm infections have been described [44, 45]. FDGPET/CT scanning has also been evaluated in patients with osteomyelitis, spondylitis and spondylodiscitis (not caused by chronic $Q$ fever). It has been shown that PET scanning offers high sensitivity and specificity [51-55]. Gratz et al. demonstrated that PET was superior in comparison to MRI imaging in spondylodiscitis [55]. Major advantage of PET/CT is that not only inflammation of vascular structures can be visualised, but also possible infectious foci in other parts of the body. It is used in the work-up of "fever of unknown origin" as an instrument to identify the origin of fever if other diagnostics have failed [56]. Therefore, it seems to be suitable for the assessment in case of a suspected chronic $Q$ fever infection where no cardiac or vascular focus is found.

In light of the issues addressed in this review, the diagnosis of chronic $Q$ fever is complex. PCR and culture are very specific, but lack sensitivity. Diagnosis based on phase I IgG alone is not sufficient to prove or exclude chronic $Q$ fever. Based on the results of the review, we believe that combining risk factors, clinical parameters, microbiology, imaging and laboratory results is obligatory to make a well considered diagnosis of chronic $Q$ fever. Although many questions have remained unanswered, with the current available literature our Dutch consensus group has composed a guideline on chronic $Q$ fever, in which chronic $Q$ fever is categorized as proven, probable or possible chronic infection (table 5). Proven chronic $Q$ fever cases are classified as positive C. burnetii PCR (or culture) in blood or tissue, or patients with phase I IgG $\geq 1: 800$ or phase I IgG $\geq 1024$ (depending on in house IFA technique or commercial IFA technique respectively), in combination with definite endocarditis according to the revised Duke criteria, or evident infection of aneurysm or vascular graft on CT, PET-CT, duplex ultrasound or MRI. In addition: diagnosis of proven chronic $Q$ fever by positive PCR can only be stated in absence of an acute infection. Positive PCR can be observed during the first two weeks of clinical symptoms of acute $Q$ fever [57]. Although most patients with chronic $Q$ fever have no known episode of acute $Q$ fever, diagnosis of chronic $Q$ fever based on phase I IgG $\geq 1: 1024$ in patients with a documented episode of acute $Q$ fever, can only be made at least six months after this episode [8, 9, 42]. Long-term antibiotic treatment, preferably consisting of hydroxychloroquine and doxycycline, should always 
be initiated in case of proven chronic $Q$ fever. Close clinical and serological follow-up is required. Further investigations such as echocardiograms or PET/CT should be considered according to the clinical picture. In refractory cases surgery should be considered. Probable chronic $Q$ fever is diagnosed in those patients with phase I IgG $\geq 1: 1024$ who have established risk factors for chronic $Q$ fever, show echocardiographic abnormalities not meeting the revised Duke criteria, probable rare manifestations of $Q$ fever (e.g. hepatitis, osteomyelitis) or signs of systemic inflammation. Frequent monitoring is warranted, consisting of three monthly clinical and microbiological follow-up. Radiographical imaging (such as echocardiogram and imaging of vascular structures, especially the aorta, for which our Consensus group recommends the use of PET /CT) should be performed whenever clinical improvement stagnates or worsens. Whether to start antibiotic treatment should be discussed in a multidisciplinary team, consisting of clinicians and microbiologists with experience in chronic $Q$ fever. Possible chronic $Q$ fever is diagnosed in patients with solely a phase I IgG $\geq 1: 1024$, without any of the manifestations mentioned in the categories proven and probable. Frequent monitoring is warranted, consisting of three monthly clinical and microbiological follow-up. Radiographical imaging (such as echocardiogram and imaging of vascular structures, especially the aorta, for which our Consensus group recommends use of PET /CT) should be performed whenever clinical improvement stagnates or worsens. In general, no antibiotic treatment should be initiated in patients with possible chronic $Q$ fever. Consequence of this guideline is that in every patient with suspected chronic $Q$ fever, an echocardiography and preferably a PET-CT has to be performed to differentiate between these categories. Our classification might need adjustment when prospective clinical data become available from the Dutch national chronic $Q$ fever monitoring system including all patients with chronic $Q$ fever diagnosed in the Netherlands (expected in 2012). For now, it is a practical tool for the diagnosis of chronic $Q$ fever and determination of an individualized treatment plan. This classification is also very important for an adequate comparison of results of future research, which proved to be very difficult in the presently available literature. 
Table 5. Dutch consensus guideline on chronic $Q$ fever diagnostics.

\begin{tabular}{|c|c|c|}
\hline Proven chronic $Q$ fever & Probable chronic $\mathbf{Q}$ fever & Possible chronic $\mathbf{Q}$ fever \\
\hline $\begin{array}{l}\text { 1. Positive C. burnetii PCR in } \\
\text { blood or tissue }{ }^{\text {a }} \\
\text { OR } \\
\text { 2. IFA } \geq 1: 1024 \text { for C. burnetii } \\
\text { phase I IgG } \\
\text { AND } \\
\text { - definite endocarditis according } \\
\text { to the modified Duke criteria [29] } \\
\text { OR } \\
\text { - proven large vessel or prosthet- } \\
\text { ic infection by imaging studies } \\
\left({ }^{18} \mathrm{FDG}-\mathrm{PET}, \mathrm{CT}, \mathrm{MRI} \text { or AUS) }\right.\end{array}$ & $\begin{array}{l}\text { IFA } \geq 1: 1024 \text { for } C \text {. burnetii } \\
\text { phase I IgG } \\
\text { AND one or more of the following } \\
\text { criteria } \\
\text { - Valvulopathy not meeting the } \\
\text { major criteria of the modified } \\
\text { Duke criteria [29] } \\
\text { - Known aneurysm and/or vascu- } \\
\text { lar or cardiac valve prosthesis } \\
\text { without signs of infection by } \\
\text { means of TEE/ TTE, }{ }^{18} \text { FDG-PET, } \\
\text { CT, MRI or abdominal doppler } \\
\text { ultrasound } \\
\text { - Suspected osteomyelitis or } \\
\text { hepatitis as manifestation of } \\
\text { chronic Q fever } \\
\text { - Pregnancy } \\
\text { - Symptoms and signs of chronic } \\
\text { infection, such as fever, weight } \\
\text { loss and night sweats, hepato- } \\
\text { splenomegaly, persistent raised } \\
\text { ESR and CRP } \\
\text { - Granulomatous tissue inflam- } \\
\text { mation, proven by histological } \\
\text { examination } \\
\text { - Immunocompromised state }\end{array}$ & $\begin{array}{l}\text { IFA } \geq 1: 1024 \text { for } C \text {. burnetii } \\
\text { phase I IgG without manifesta- } \\
\text { tions meeting the criteria for } \\
\text { proven or probable chronic } Q \\
\text { fever }\end{array}$ \\
\hline
\end{tabular}

Abbreviations: CT, computer tomography; IFA, immunofluorescence assay; TEE, transesophageal echocardiography; TTE, transthoracic echocardiography.

${ }^{a}$ In absence of acute infection. 
** Members of the Dutch Q fever consensus Group

H.A. Bijlmer, chairman (medical microbiologist) ${ }^{6}$, C. Bleeker-Rovers (infectious diseases specialist) ${ }^{3}$, C.E. Delsing (infectious diseases specialist) ${ }^{3}$, J.M.D. Galama (medical microbiologist) ${ }^{3}$, T. Herremans (biologist) ${ }^{6}$, B.M. Hogema (biologist) ${ }^{7}$, A.M. Horrevorts (medical microbiologist) ${ }^{4}$, L.M. Kampschreur (resident infectious diseases specialist) ${ }^{2}, M$. van Kasteren (infectious diseases specialist) $^{8}$, M.P.G. Koopmans (virologist) ${ }^{6}$, P. Lestrade (internist) ${ }^{5}$, I.H.M. van Loo (medical microbiologist) ${ }^{9}$, J. Marcelis (medical microbiologist) ${ }^{8}$, M.H. Nabuurs-Franssen (medical microbiologist) ${ }^{3,4}$, D.W. Notermans (medical microbiologist) ${ }^{6}$, J.J. Oosterheert (infectious diseases specialist) ${ }^{2}$, N.H.M Renders (medical microbiologist) ${ }^{5}$, P.M. Schneeberger (medical microbiologist $)^{5}, \mathrm{~T}$. Sprong (infectious diseases specialist) ${ }^{4}$, J. van Steenbergen (epidemiologist) ${ }^{6}$, J.A.W. Teijink (vascular surgeon) ${ }^{10}$, B.J. Vlaminckx (medical microbiologist) ${ }^{11}$, P.H. van der Voort (cardiologist) ${ }^{10}$, J.P. de Vries (vascular surgeon) ${ }^{11}$, M.C.A. Wegdam-Blans (medical microbiologist $^{1,10}$, P.C. Wever (medical microbiologist) ${ }^{5}$ and H.L. Zaaijer (medical microbiologist $)^{7}$.

${ }^{1}$ Dept. of medical Microbiology, Laboratory of Pathology and Medical Microbiology (PAMM) Veldhoven, ${ }^{2}$ University Medical Center Utrecht, ${ }^{3}$ Radboud University Nijmegen Medical Center Nijmegen, ${ }^{4}$ Canisius Wilhelmina Hospital Nijmegen, ${ }^{5}$ Jeroen Bosch Hospital 's Hertogenbosch, ${ }^{6}$ Centre for Infectious Disease Control, National Institute for Public Health and the Environment (RIVM), Bilthoven, ${ }^{7}$ Sanquin Amsterdam, ${ }^{8}$ St Elisabeth Hospital Tilburg, ${ }^{9}$ University Medical Center Maastricht, ${ }^{10}$ Catharina Hospital Eindhoven, ${ }^{11}$ St Antonius Hospital Nieuwegein.

The Dutch consensus guideline on chronic $Q$ fever diagnostics was presented at the $6^{\text {th }}$ International Meeting on Rickettsiae and Rickettsial Diseases held in Heraklion in June 2011. 


\section{References}

1. Parker NR, Barralet JH, Bell AM. Q fever. Lancet 2006; 367(9511):679-88.

2. Raoult $D$, Marrie $T$, Mege J. Natural history and pathophysiology of $Q$ fever. Lancet Infect Dis 2005; 5(4):219-26.

3. Tissot-Dupont H, Raoult D. Q fever. Infect Dis Clin North Am 2008; 22(3):505-14, ix.

4. Botelho-Nevers E, Fournier PE, Richet $\mathrm{H}$, et al. Coxiella burnetii infection of aortic aneurysms or vascular grafts: report of 30 new cases and evaluation of outcome. Eur $\mathrm{J}$ Clin Microbiol Infect Dis 2007; 26(9):635-40.

5. Million M, Thuny F, Richet H, Raoult D. Long-term outcome of $Q$ fever endocarditis: a 26year personal survey. Lancet Infect Dis 2010; 10(8):527-35.

6. Brouqui $P$, Dupont HT, Drancourt $M$, et al. Chronic $Q$ fever. Ninety-two cases from France, including 27 cases without endocarditis. Arch Intern Med 1993; 153(5):642-8.

7. Raoult D, Tissot-Dupont H, Foucault C, et al. Q fever 1985-1998. Clinical and epidemiologic features of 1,383 infections. Medicine (Baltimore) 2000; 79(2):109-23.

8. Frankel D, Richet H, Renvoise A, Raoult D. Q fever in France, 1985-2009. Emerg Infect Dis 2011; 17(3):350-6.

9. Fenollar F, Fournier PE, Carrieri MP, Habib G, Messana T, Raoult D. Risks factors and prevention of $Q$ fever endocarditis. Clin Infect Dis 2001; 33(3):312-6.

10. Landais $C$, Fenollar $F$, Thuny $F$, Raoult $D$. From acute $Q$ fever to endocarditis: serological follow-up strategy. Clin Infect Dis 2007; 44(10):1337-40.

11. Raoult D, Houpikian P, Tissot DH, Riss JM, rditi-Djiane J, Brouqui P. Treatment of $Q$ fever endocarditis: comparison of 2 regimens containing doxycycline and ofloxacin or hydroxychloroquine. Arch Intern Med 1999; 159(2):167-73.

12. Musso D, Raoult D. Coxiella burnetii blood cultures from acute and chronic Q-fever patients. J Clin Microbiol 1995; 33(12):3129-32.

13. Fenollar F, Fournier PE, Raoult D. Molecular detection of Coxiella burnetii in the sera of patients with Q fever endocarditis or vascular infection. J Clin Microbiol 2004; 42(11):4919-24.

14. Dupuis G, Peter O, Luthy R, Nicolet J, Peacock M, Burgdorfer W. Serological diagnosis of Q fever endocarditis. Eur Heart J 1986; 7(12):1062-6.

15. Dupont HT, Thirion X, Raoult D. Q fever serology: cutoff determination for microimmunofluorescence. Clin Diagn Lab Immunol 1994; 1(2):189-96.

16. Healy $B$, van Woerden $H$, Raoult $D$, et al. Chronic $Q$ fever: different serological results in three countries--results of a follow-up study 6 years after a point source outbreak. Clin Infect Dis 2011; 52(8):1013-9.

17. Hung MN, Lin LJ, Hou MY, et al. Serologic assessment of the risk of developing chronic $Q$ fever in cohorts of acutely infected individuals. J Infect 2011; 62(1):39-44.

18. van der Hoek W, Dijkstra F, Schimmer B, et al. Q fever in the Netherlands: an update on the epidemiology and control measures. Euro Surveill 2010; 15(12) pii=19520, http://www.eurosurveillance.org/ViewArticle.aspx?Articleld=19520.

19. Delsing CE, Kullberg BJ, Bleeker-Rovers CP. Q fever in the Netherlands from 2007 to 2010. Neth J Med 2010; 68(12):382-7.

20. Wiener-Well Y, Fink D, Schlesinger Y, Raveh D, Rudensky B, Yinnon AM. Q fever endocarditis; not always expected. Clin Microbiol Infect 2010; 16(4):359-62. 
21. Kokkini S, Kofteridis D, Psaroulaki A, et al. Q fever endocarditis in Greece: report of five cases. Clin Microbiol Infect 2009; 15 Suppl 2:136-7.

22. Scott JW, Baddour LM, Tleyjeh IM, Moustafa S, Sun YG, Mookadam F. Q fever endocarditis: the Mayo Clinic experience. Am J Med Sci 2008; 336(1):53-7.

23. Salamand AC, Collart F, Caus $T$, et al. $Q$ fever endocarditis: over 14 years of surgical experience in a referral center for rickettsioses. J Heart Valve Dis 2002; 11(1):84-90.

24. Boyle B, Hone R. Q fever endocarditis revisited. Ir J Med Sci 1999; 168(1):53-4.

25. Tobin MJ, Cahill N, Gearty G, et al. Q fever endocarditis. Am J Med 1982; 72(3):396-400.

26. Rolain JM, Lecam C, Raoult D. Simplified serological diagnosis of endocarditis due to Coxiella burnetii and Bartonella. Clin Diagn Lab Immunol 2003; 10(6):1147-8.

27. Lepidi H, Houpikian P, Liang Z, Raoult D. Cardiac valves in patients with $Q$ fever endocarditis: microbiological, molecular, and histologic studies. J Infect Dis 2003; 187(7):1097106.

28. Brouqui P, Dumler JS, Raoult D. Immunohistologic demonstration of Coxiella burnetii in the valves of patients with $Q$ fever endocarditis. Am J Med 1994; 97(5):451-8.

29. Li JS, Sexton DJ, Mick N, et al. Proposed modifications to the Duke criteria for the diagnosis of infective endocarditis. Clin Infect Dis 2000; 30(4):633-8.

30. Soriano F, Camacho MT, Ponte C, Gomez P. Serological differentiation between acute (late control) and endocarditis Q fever. J Clin Pathol 1993; 46(5):411-4.

31. Peter O, Flepp M, Bestetti G, Nicolet J, Luthy R, Dupuis G. Q fever endocarditis: diagnostic approaches and monitoring of therapeutic effects. Clin Investig 1992; 70(10):932-7.

32. Edlinger EA. Chronic Q fever. Zentralbl Bakteriol Mikrobiol Hyg A 1987; 267(1):51-6.

33. Peacock MG, Philip RN, Williams JC, Faulkner RS. Serological evaluation of $O$ fever in humans: enhanced phase I titers of immunoglobulins $G$ and $A$ are diagnostic for $Q$ fever endocarditis. Infect Immun 1983; 41(3):1089-98.

34. Worswick D, Marmion BP. Antibody responses in acute and chronic $Q$ fever and in subjects vaccinated against $Q$ fever. J Med Microbiol 1985; 19(3):281-96.

35. Kowalczewska M, Sekeyova Z, Raoult D. Proteomics paves the way for $Q$ fever diagnostics. Genome Med 2011; 3(7):50, http://genomemedicine.com/content/3/7/50.

36. Vigil A, Chen C, Jain A, et al. Profiling the humoral immune response of acute and chronic Q fever by protein microarray. Mol Cell Proteomics 2011, doi: 10.1074/mcp.M110.006304.

37. Sekeyova Z, Kowalczewska M, Vincentelli R, et al. Characterization of antigens for $Q$ fever serodiagnostics. Acta Virol 2010; 54(3):173-80.

38. Sekeyova Z, Kowalczewska M, Decloquement P, Pelletier N, Spitalska E, Raoult D. Identification of protein candidates for the serodiagnosis of $Q$ fever endocarditis by an immunoproteomic approach. Eur J Clin Microbiol Infect Dis 2009; 28(3):287-95.

39. Ake JA, Massung RF, Whitman TJ, Gleeson TD. Difficulties in the diagnosis and management of a US servicemember presenting with possible chronic $Q$ fever. J Infect 2010; 60(2):175-7.

40. van der Hoek W, Versteeg B, Meekelenkamp JC, et al. Follow-up of 686 Patients With Acute Q Fever and Detection of Chronic Infection. Clin Infect Dis 2011; 52(12):1431-6.

41. Sunder S, Gras G, Bastides F, De GC, Choutet P, Bernard L. Chronic Q fever: relevance of serology. Clin Infect Dis 2011; 53(7):749-50.

42. Wegdam-Blans MC, Vainas $T$, van Sambeek MR, et al. Vascular Complications of Q-fever Infections. Eur J Vasc Endovasc Surg 2011; 42(3):384-92. 
43. Limonard GJ, Peters JB, Nabuurs-Franssen MH, et al. Detailed analysis of health status of $Q$ fever patients 1 year after the first Dutch outbreak: a case-control study. QJM 2010; 103(12):953-8.

44. van Assen S, Houwerzijl EJ, van den Dungen JJ, Koopmans KP. Vascular graft infection due to chronic $Q$ fever diagnosed with fusion positron emission tomography/computed tomography. J Vasc Surg 2007; 46(2):372.

45. Bendermacher BL, Peppelenbosch AG, Daemen JW, Oude Lashof AM, Jacobs MJ. Q fever (Coxiella burnetii) causing an infected thoracoabdominal aortic aneurysm. J Vasc Surg 2011; 53(5):1402-4.

46. Bruggink JL, Glaudemans AW, Saleem BR, et al. Accuracy of FDG-PET-CT in the diagnostic work-up of vascular prosthetic graft infection. Eur J Vasc Endovasc Surg 2010; 40(3):348-54.

47. Keidar Z, Nitecki S. FDG-PET for the detection of infected vascular grafts. Q J Nucl Med Mol Imaging 2009; 53(1):35-40.

48. Fukuchi K, Ishida $\mathrm{Y}$, Higashi $\mathrm{M}$, et al. Detection of aortic graft infection by fluorodeoxyglucose positron emission tomography: comparison with computed tomographic findings. J Vasc Surg 2005; 42(5):919-25.

49. Spacek M, Belohlavek O, Votrubova J, Sebesta P, Stadler P. Diagnostics of "non-acute" vascular prosthesis infection using 18F-FDG PET/CT: our experience with 96 prostheses. Eur J Nucl Med Mol Imaging 2009; 36(5):850-8.

50. Orton DF, LeVeen RF, Saigh JA, et al. Aortic prosthetic graft infections: radiologic manifestations and implications for management. Radiographics 2000; 20(4):977-93.

51. Kalicke T, Schmitz A, Risse $\mathrm{JH}$, et al. Fluorine-18 fluorodeoxyglucose PET in infectious bone diseases: results of histologically confirmed cases. Eur J Nucl Med 2000; 27(5):5248.

52. Meller J, Koster G, Liersch T, et al. Chronic bacterial osteomyelitis: prospective comparison of (18)F-FDG imaging with a dual-head coincidence camera and (111)In-labelled autologous leucocyte scintigraphy. Eur J Nucl Med Mol Imaging 2002; 29(1):53-60.

53. De Winter F, Van de Wiele C, Vogelaers D, de Smet K, Verdonk R, Dierckx RA. Fluorine18 fluorodeoxyglucose-position emission tomography: a highly accurate imaging modality for the diagnosis of chronic musculoskeletal infections. J Bone Joint Surg Am 2001; 83$A(5): 651-60$.

54. De Winter F, Gemmel F, Van de Wiele C, Poffijn B, Uyttendaele D, Dierckx R. 18-Fluorine fluorodeoxyglucose positron emission tomography for the diagnosis of infection in the postoperative spine. Spine (Phila Pa 1976 ) 2003; 28(12):1314-9.

55. Gratz S, Dorner J, Fischer U, et al. 18F-FDG hybrid PET in patients with suspected spondylitis. Eur J Nucl Med Mol Imaging 2002; 29(4):516-24.

56. Gotthardt M, Bleeker-Rovers CP, Boerman OC, Oyen WJ. Imaging of inflammation by PET, conventional scintigraphy, and other imaging techniques. J Nucl Med 2010; 51(12):1937-49.

57. Schneeberger PM, Hermans MH, van Hannen EJ, Schellekens JJ, Leenders AC, Wever PC. Real-time PCR with serum samples is indispensable for early diagnosis of acute $Q$ fever. Clin Vaccine Immunol 2010; 17(2):286-90. 




\section{Chapter 5}

\section{Chronic $Q$ fever diagnosis: consensus guideline versus expert opinion}

Submitted for publication

L.M. Kampschreur

M.C.A. Wegdam-Blans

P.C. Wever

N.H.M. Renders

C.E. Delsing

T. Sprong

M.E.E. van Kasteren

H.A. Bijlmer

D. Notermans

J.J. Oosterheert

F.S. Staals

M.H. Nabuurs-Franssen

C.P. Bleeker-Rovers 



\section{Abstract}

Chronic $Q$ fever, caused by Coxiella burnetii, is an infection with high mortality and morbidity if left untreated. Controversy about the diagnosis of the complex disease has emerged recently. We applied both the Dutch consensus guideline from the Dutch $Q$ Fever Consensus Group and the diagnostic criteria proposed by the leading expert in $Q$ fever, professor Raoult from France, to all chronic $Q$ fever patients included in the Dutch National Chronic $Q$ Fever Database. Of the cases with proven chronic $Q$ fever according to the Dutch guideline, $31 \%$ would be left undiagnosed with Raoult's guideline. Four patients with chronic $Q$ fever based on the Dutch consensus guideline but without chronic $Q$ fever according to the guideline proposed by Raoult died of chronic $Q$ fever-related causes. High sensitivity is of major importance in the diagnosis of chronic $Q$ fever because mortality of untreated chronic $Q$ fever is high. Until results from future studies are available by which current guidelines can be modified to definite evidence-based diagnostic criteria for this complex disease, it is our opinion that the Dutch literature-based consensus guideline is safer and easier to use in clinical practice than Raoult's expert-based guideline. 


\section{Introduction}

Coxiella burnetii ( $C$. burnetii) is the causative agent of $Q$ fever, a zoonosis occurring worldwide [1]. Recently there has been a large epidemic in the Netherlands with over 4000 cases of acute Q fever notified between 2007 and 2010 [2, 3]. Chronic $Q$ fever develops in an estimated 1-5\% of all infected humans and can become manifest even years after primary infection [1, 4]. Endocarditis and infection of aneurysms or vascular prostheses are the most common manifestations $[1,5,6]$.

Untreated chronic $Q$ fever has a poor prognosis, with a reported mortality rate of up to $60 \%[7,1]$. Adequate antibiotic treatment reduces mortality of $Q$ fever endocarditis to less than 5\% [7]. Treatment preferably consists of a combination of doxycycline and hydroxychloroquine for at least 18 months (non-prosthetic infection) to 24 months (prosthetic infection) and is advised to be continued in case of unfavourable clinical or serologic response [7, 8]. Antibiotic guidelines for vascular chronic $Q$ fever are not available yet, but antibiotic regimes for $Q$ fever endocarditis have been applied to this disease entity as well. Early surgical intervention, with removal of infected material might improve the diagnosis of vascular chronic $Q$ fever $[9,6]$. In the early course of chronic $Q$ fever, most patients are asymptomatic or experience non-specific symptoms such as low-grade fever, night sweats, and weight loss $[6,4,1,7]$. Findings on echocardiography are often non-specific or absent in case of endocarditis, which makes the diagnosis of chronic $Q$ fever challenging [7].

A positive polymerase chain reaction (PCR) or culture of $C$. burnetii in blood or tissue, in the absence of acute $Q$ fever, is a strong indicator for chronic $Q$ fever. However, sensitivity on blood samples is only $50-60 \%$ for both PCR and culture in patients with chronic $Q$ fever $[10,11]$. Therefore, serology is also important for the diagnosis of chronic $Q$ fever. A phase I IgG cut-off titer of 1:800, which is based on an in-house developed immunofluorescence assay (IFA), has been internationally accepted for the diagnosis of chronic $Q$ fever and is included in the modified Duke criteria for diagnosis of endocarditis [12, 13]. In the Netherlands, a commercial IFA (Focus Diagnostics) is mostly used, with a proposed cut-off value of 1:1024 for chronic $Q$ fever [14]. Yet, recent studies show that serology results alone are not sufficient for the diagnosis of chronic $Q$ fever, but should be combined with clinical data [15]. 
Faced with a large $Q$ fever outbreak in the Netherlands and a rising number of (presumed) chronic $Q$ fever patients, we were not able to find answers to all our questions about this complex disease in the literature. Moreover, randomized trials on diagnosis and treatment of this disease were lacking and available data were not all applicable to the Dutch situation. For example, we found far more vascular localizations of chronic $Q$ fever, with often severe complications, than described previously. Therefore, the Dutch $Q$ Fever Consensus Group was initiated, in which diagnosis and subsequent treatment consequences for suspected chronic $Q$ fever were discussed. We performed a thorough literature review and constructed a new guideline for the diagnosis of chronic $Q$ fever, differentiating between proven, probable and possible chronic $Q$ fever (table 1). We added advice for treatment and follow-up regimes for these three groups of patients. Antibiotic treatment and, if indicated, surgical treatment are recommended for all patients with proven chronic $Q$ fever. The decision to start antibiotic treatment in patients with probable chronic $Q$ fever depends on clinical characteristics and the condition of the patient, and should be discussed in a multidisciplinary team. In possible chronic $\mathrm{Q}$ fever patients, antibiotic treatment should not be initiated, but follow-up is indicated.

The Dutch consensus guideline was recently published [14]. Subsequently a reaction of professor Raoult was published, opposing our proposed guideline and formulating another guideline based on his expert opinion (table 2) [16]. Professor Raoult is the undisputed leading expert in $Q$ fever and his expert opinion and scientific publications from his research group should be considered by anyone working in the field of $Q$ fever. Here, we reflect on this controversy by applying both guidelines to cases from the Dutch National Chronic Q Fever Database.

\section{Diagnosis of chronic $\mathbf{Q}$ fever: literature-based consensus guideline versus expert opinion}

Raoult opposes to the term "chronic $Q$ fever", but makes a distinction in two manifestations: $Q$ fever endocarditis and $Q$ fever vascular infection. This distinction is not accompanied by therapeutic consequences for each of these manifestations. Moreover, more rare manifestations, like pericarditis, hepatitis, and osteomyelitis are left undefined. 
Table 1. Dutch consensus guideline on chronic $Q$ fever diagnostics [14].

\begin{tabular}{|c|c|c|}
\hline Proven chronic $Q$ fever & Probable chronic $Q$ fever & Possible chronic $\mathbf{Q}$ fever \\
\hline $\begin{array}{l}\text { 1. Positive C. burnetii PCR in } \\
\text { blood or tissue }{ }^{\mathrm{a}} \\
\text { OR } \\
\text { 2. IFA } \geq 1: 1024 \text { for C. burnetii } \\
\text { phase I IgG }{ }^{\mathrm{b}} \\
\text { AND } \\
\text { - definite endocarditis according } \\
\text { to the modified Duke criteria [13] } \\
\text { OR } \\
\text { - proven large vessel or prosthet- } \\
\text { ic infection by imaging studies } \\
\left({ }^{18} \text { FDG-PET, CT, MRI or AUS) }\right.\end{array}$ & $\begin{array}{l}\text { IFA } \geq 1: 1024 \text { for } C \text {. burnetii } \\
\text { phase I IgG }{ }^{\mathrm{b}} \\
\text { AND one or more of the following } \\
\text { criteria } \\
\text { - Valvulopathy not meeting the } \\
\text { major criteria of the modified } \\
\text { Duke criteria [13] } \\
\text { - Known aneurysm and/or vascu- } \\
\text { lar or cardiac valve prosthesis } \\
\text { without signs of infection by } \\
\text { means of TEE/ TTE, }{ }^{18} \text { FDG-PET, } \\
\text { CT, MRI or abdominal doppler } \\
\text { ultrasound } \\
\text { - Suspected osteomyelitis or } \\
\text { hepatitis as manifestation of } \\
\text { chronic Q fever } \\
\text { - Pregnancy } \\
\text { - Symptoms and signs of chronic } \\
\text { infection, such as fever, weight } \\
\text { loss and night sweats, hepato- } \\
\text { splenomegaly, persistent raised } \\
\text { ESR and CRP } \\
\text { - Granulomatous tissue inflam- } \\
\text { mation, proven by histological } \\
\text { examination } \\
\text { - Immunocompromised state }\end{array}$ & $\begin{array}{l}\text { IFA } \geq 1: 1024 \text { for } C \text {. burnetii } \\
\text { phase I lgG }{ }^{b} \text { without manifesta- } \\
\text { tions meeting the criteria for } \\
\text { proven or probable chronic } Q \\
\text { fever }\end{array}$ \\
\hline
\end{tabular}

Abbreviations: IFA, immunofluorescence assay; FDG-PET, fluorodeoxyglucose positron emission tomography; CT, computer tomography; MRI, magnetic resonance imaging; AUS, abdominal ultrasound; TEE, transesophageal echocardiography; TTE, transthoracic echocardiography.

${ }^{a}$ In absence of acute infection.

${ }^{\mathrm{b}}$ Cut-off is depending on the IFA technique used, respectively, in house developed or commercial IFA technique.

An important difference in the diagnostic criteria proposed by Raoult and the Dutch $Q$ Fever Consensus Group is the diagnostic value attributed to $C$. burnetii PCR positivity of blood samples. Being unaware of other clinical entities presenting with a positive $C$. burnetii PCR in blood than acute and chronic $Q$ fever, we believe that a positive blood PCR, in the absence of acute $Q$ fever, proves chronic $Q$ fever. Raoult on the other hand states that a positive PCR in blood should be accompanied by a clear endocarditis focus on echocardiography, a clear vascular focus on imaging studies, or at least two or three "minor criteria" (table 2). Moreover, great value is attributed by Raoult to the phase I IgG titer proposing a phase I IgG $\geq 1: 6400$ as major criterion for $Q$ fever endocarditis and $Q$ fever vascular infection, opposed to a phase I IgG $\geq 1: 800$ and $<1: 6400$ being proposed as a minor criterion. This 
is in contradiction to the internationally accepted modified Duke criteria, in which a phase I IgG $\geq 1: 800$ is stated as a major criterion for infective ( $Q$ fever) endocarditis [13].

Table 2. Diagnostic guideline for chronic $Q$ fever proposed by Raoult [16].

2A. $Q$ fever endocarditis

\section{A. Definite Criteria}

Positive culture, PCR, or immunochemistry of a cardiac valve

\section{B. Major Criteria}

Microbiology: positive culture or PCR of the blood or an emboli or serology with IgGI antibodies $\geq 6400$

Evidence of endocardial involvement:

- Echocardiogram positive for IE: oscillating intra-cardiac mass on valve or supporting structure, in the path of regurgitant jets, or on implanted material in the absence of an alternative anatomic explanation; or abscess; or new partial dehiscence of prosthetic vale; or new valvular regurgitation (worsening or changing of pre-existent murmur not sufficient)

- PET-scan showing a specific valve fixation and mycotic aneurysm

\section{Minor criteria}

Predisposing heart condition (know or found on echocardiography)

Fever, temperature $>38^{\circ} \mathrm{C}$

Vascular phenomena, major arterial emboli, septic pulmonary infarcts, mycotic aneurysm (see at PETscan), intracranial hemorrhage, conjunctival hemorrhages, and Janeway's lesions

Immunological phenomena: glomerulonephritis, Osler's nodes, Roth spots, or rheumatoid factor

Serological evidence: IgGI antibodies $\geq 800<6400$

Diagnosis definite

1. $1 \mathrm{~A}$ criterion

2. $2 \mathrm{~B}$ criterion

3. $1 \mathrm{~B}$, and $3 \mathrm{C}$ criterion

Diagnosis possible

1. $1 \mathrm{~B}$ criterion, $2 \mathrm{C}$ criteria (including microbiology evidence, and cardiac predisposition)

2. $3 \mathrm{C}$ criteria (including positive serology, and cardiac predisposition) 
Table 2. Continued.

2B. $Q$ fever vascular infection

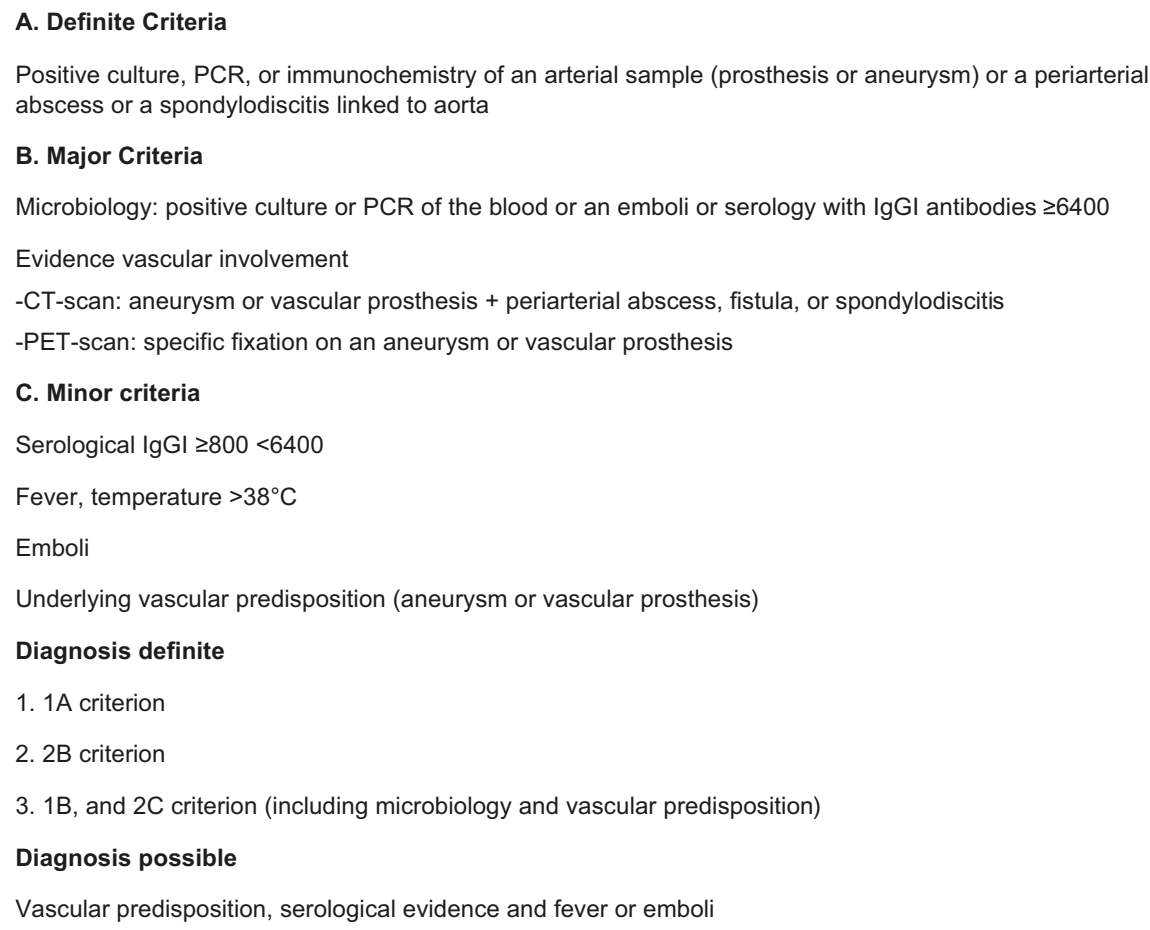

Positive culture, PCR, or immunochemistry of an arterial sample (prosthesis or aneurysm) or a periarterial abscess or a spondylodiscitis linked to aorta

\section{B. Major Criteria}

Microbiology: positive culture or PCR of the blood or an emboli or serology with IgGI antibodies $\geq 6400$

Evidence vascular involvement

-CT-scan: aneurysm or vascular prosthesis + periarterial abscess, fistula, or spondylodiscitis

-PET-scan: specific fixation on an aneurysm or vascular prosthesis

C. Minor criteria

Serological lgGI $\geq 800<6400$

Fever, temperature $>38^{\circ} \mathrm{C}$

Emboli

Underlying vascular predisposition (aneurysm or vascular prosthesis)

Diagnosis definite

1. $1 \mathrm{~A}$ criterion

2. $2 \mathrm{~B}$ criterion

3. $1 \mathrm{~B}$, and $2 \mathrm{C}$ criterion (including microbiology and vascular predisposition)

Diagnosis possible

Vascular predisposition, serological evidence and fever or emboli

After the recent outbreak of $Q$ fever in the Netherlands we initiated the "Dutch National Chronic Q Fever Database", which is a joint effort of multiple hospitals in the $Q$ fever afflicted areas to monitor all chronic $Q$ fever cases in the Netherlands. All hospitals with chronic $Q$ fever patients, also outside the notified $Q$ fever epidemic areas, were actively approached. Design of the database and use of the collected information for analysis and scientific publications were approved by the Medical Research Ethics Committee of the University Medical Center Utrecht in Utrecht, the Netherlands. Part of these data were already published in a report discussing serologic profiles of patients with chronic $Q$ fever [15].

Until the end of May 2012, 284 patients were included in our database: 151 patients (53.7\%) had proven chronic Q fever, while 64 patients $(22.5 \%)$ and 69 patients $(24.3 \%)$ had probable and possible chronic $Q$ fever according to the Dutch consensus guideline, respectively. We re-evaluated these chronic $Q$ fever cases with the diagnostic criteria proposed by Raoult (table 3 ). Of the cases with proven chronic $Q$ fever according to the Dutch guideline, 46 
patients $(30.5 \%)$ would be left undiagnosed with Raoult's guideline. For probable chronic $Q$ fever this would be 58 cases $(90.6 \%)$ and for possible chronic $Q$ fever cases all patients. Eight patients with proven chronic $Q$ fever based on PCR-positivity in blood and suspicion of endocarditis would be diagnosed with possible $Q$ fever endocarditis only by Raoult's guideline (table 4) and 18 patients with proven chronic $Q$ fever would not be diagnosed with $Q$ fever endocarditis at all, because echocardiography results did not match any major clinical Duke criterion as is often observed in $Q$ fever endocarditis [7]. Of the eight proven chronic $Q$ fever endocarditis patients (Dutch consensus guideline) who were diagnosed with possible endocarditis according to Raoult's guideline, two would be defined as definite endocarditis by the modified Duke criteria [13].

Table 3. Comparison of chronic $Q$ fever diagnosis according to the Dutch consensus guideline [14] and the guideline proposed by Raoult [16].

\begin{tabular}{|c|c|c|c|}
\hline \multirow[b]{2}{*}{ Raoult's guideline } & \multicolumn{3}{|c|}{ Dutch consensus guideline } \\
\hline & $\begin{array}{l}\text { Proven chronic Q } \\
\text { fever, } n=151(\%)\end{array}$ & $\begin{array}{l}\text { Probable chronic } Q \\
\text { fever, } n=64(\%)\end{array}$ & $\begin{array}{l}\text { Possible chronic } Q \\
\text { fever, } n=69(\%)\end{array}$ \\
\hline Definite $Q$ fever endocarditis & $21(13.9)$ & 0 & 0 \\
\hline Possible Q fever endocarditis & $8(5.3)$ & $4(6.3)$ & 0 \\
\hline Definite $Q$ fever vascular infection & $76(50.3)$ & 0 & 0 \\
\hline Possible $Q$ fever vascular infection & 0 & $2(3.1)$ & 0 \\
\hline No diagnosis of chronic $Q$ fever & $46(30.5)$ & $58(90.6)$ & $69(100.0)$ \\
\hline
\end{tabular}

Twenty-four patients with a vascular $Q$ fever infection (Dutch consensus guideline) would not be diagnosed with chronic $Q$ fever using Raoult's criteria (table 4). Seventeen of these patients had a fluorodeoxyglucose positron emission tomography-computer tomography (FDG-PET/CT) positive vascular lesion with phase I IgG $\geq 1: 800$ and $<1: 6400$. Seven patients had a positive PCR in blood in combination with an aneurysm or vascular prosthesis but no signs of infection on FDG-PET/CT. There were five proven chronic $Q$ fever patients according to the Dutch consensus guideline with no known focus and two patients with another focus than endocarditis or vascular infection who would have been missed with Raoult's criteria: five of them (repeatedly) had a positive PCR in blood but no clear infectious focus on echocardiography and FDG-PET/CT. One patient had a positive PCR in blood 
with clinical pericarditis, and one patient had a positive PCR in blood during pregnancy with phase I IgG >1:1024 and a positive PCR of placental tissue.

Table 4. Characteristics and outcome of patients diagnosed with chronic $Q$ fever using the Dutch consensus guideline [14] but without (definite) chronic $Q$ fever according to the guideline proposed by Raoult [16].

\begin{tabular}{|c|c|c|}
\hline \multirow[b]{3}{*}{ Dutch consensus guide line } & \multicolumn{2}{|l|}{ Raoult's guideline } \\
\hline & $\begin{array}{l}\text { Possible } Q \text { fever } \\
\text { endocarditis or vascu- } \\
\text { lar infection }\end{array}$ & $\begin{array}{l}\text { No diagnosis } \\
n=173(\%)\end{array}$ \\
\hline & $n=14(\%)$ & \\
\hline Proven $Q$ fever & $8(57.1)$ & $46(26.6)$ \\
\hline Endocarditis & $8(57.1)$ & $18(10.4)^{a}$ \\
\hline PCR positive in blood & $6(42.9)$ & $18(10.4)$ \\
\hline Evidence of endocardial involvement & $2(14.3)$ & 0 \\
\hline Vascular infection & 0 & $24(13.9)^{\mathrm{a}}$ \\
\hline PCR positive in blood & 0 & $7(4.0)$ \\
\hline Vascular focus on imaging & 0 & $17(9.8)$ \\
\hline Other or no focus (all PCR in blood positive) & 0 & $7(4.1)$ \\
\hline Deceased & $2(14.3)$ & $8(4.6)$ \\
\hline Death probably due to $Q$ fever & $2(14.3)$ & $4(2.3)^{b}$ \\
\hline Probable $Q$ fever & $6(42.9)$ & $58(33.5)$ \\
\hline Endocarditis & $4(28.6)$ & $22(12.7)$ \\
\hline Vascular infection & $2(14.3)$ & $16(9.3)$ \\
\hline Other or no focus & 0 & $20(11.6)$ \\
\hline Deceased & $2(14.3)$ & $4(2.3)$ \\
\hline Death probably due to $Q$ fever & $1(7.1)$ & 0 \\
\hline Possible $Q$ fever & 0 & $69(39.9)$ \\
\hline
\end{tabular}

${ }^{a}$ In three proven chronic $Q$ fever patients imaging studies revealed that the focus of infection was both on the heart valves and vascular structures.

${ }^{\mathrm{b}}$ Two patients with positive C. burnetii PCR on vascular and heart valve tissue obtained at autopsy.

Importantly, ten proven chronic $Q$ fever patients who were not diagnosed with definite chronic $Q$ fever using Raoult's criteria died (two with possible chronic $Q$ fever and eight without chronic $Q$ fever according to Raoult's guideline). Six of these patients died due to clear chronic $Q$ fever related manifestations (two with possible chronic $Q$ fever and four without chronic $Q$ fever according to Raoult's guideline, table 4). The two patients with possible chronic $Q$ fever died of complications caused by endocarditis, one of them had a double-pathogen endocarditis with Staphylococcus aureus. Two 
of the four patients without chronic $Q$ fever according to Raoult's guideline died due to aorta-duodenal fistula, both with a phase I IgG >1:1024, but $<1: 6400$, negative PCR on blood, and a clear FDG-positive vascular focus on PET/CT. In one of these two patients, $Q$ fever vascular infection was confirmed post-mortem with a positive PCR of the abdominal aortic aneurysm. No autopsy was performed on the other patient unfortunately. The third patient with a history of a biological heart valve replacement, an FDGPET/CT negative aortic aneurysm, and a positive PCR on blood eventually died of heart failure. Post-mortem analysis demonstrated a positive PCR of the heart valve confirming $Q$ fever endocarditis. Another chronic $Q$ fever patient with positive blood PCR and minor valve lesions according to the Duke criteria died of gastro-intestinal bleeding probably due to aortaintestinal fistula.

\section{Conclusions}

There are several major differences between the Dutch consensus guideline for the diagnosis of chronic $Q$ fever and the guideline proposed by Raoult. Sensitivity of the Dutch guideline is higher. With Raoult's criteria approximately $30 \%$ of proven chronic $Q$ fever cases would be missed and almost all probable and possible cases, including at least four patients that eventually died due to chronic $Q$ fever related causes. The number of deaths might even have been higher if fewer patients with chronic $Q$ fever were treated as would be the case when Raoult's guidelines would have been applied to the Dutch chronic $Q$ fever patients. Specificity of the Dutch consensus guideline is probably lower, but as untreated chronic $Q$ fever has high mortality and high morbidity, we believe sensitivity is of greater importance in clinical practice. Missing and therefore not adequately treating proven chronic $Q$ fever possesses a high risk for severe complications and death in these patients as was illustrated with our data. In our opinion, patients without endocarditis or vascular infection on imaging studies, but with a positive PCR in blood should also be treated for chronic $Q$ fever, as they may suffer from not yet clinically visible endocarditis or vascular infection, which was confirmed by the post-mortem results of two of our patients described above. We agree with the statement that part of the patients with probable chronic $Q$ fever and most patients with possible chronic $Q$ fever will eventually not have chronic $Q$ fever. We therefore do not advocate treating all of these patients with 
long-term antibiotics. Nevertheless, we do think that these patients should all be analysed for a chronic $Q$ fever focus and should remain under close follow-up, at least until further research offers more clarity to the prognosis of these patients. If these patients are not diagnosed with possible or probable chronic $Q$ fever, they might not receive such close follow-up. Moreover, the Dutch consensus guideline is easier to use, adds treatment advice, and also applies to patients with rarer chronic $Q$ fever manifestations other than endocarditis and vascular infection. We hope that with the future results of the Dutch National Chronic Q Fever Database and joint efforts of international researchers and experts in the field of $Q$ fever these guidelines can be modified to definite evidence-based criteria for this complex disease. In the meantime the Dutch consensus guideline based on the available literature is in our opinion safer and easier to use in clinical practice than Raoult's expert-based guideline. 


\section{References}

1. Maurin M, Raoult D. Q fever. Clin Microbiol Rev 1999; 12: 518-53.

2. van der Hoek W, Dijkstra F, Schimmer B, et al. Q fever in the Netherlands: an update on the epidemiology and control measures. Euro Surveill 2010; 15: http://www.eurosurveillance.org/ViewArticle.aspx?Articleld=19520. Accessed at 25th July 2012.

3. Delsing CE, Kullberg BJ, Bleeker-Rovers CP. Q fever in the Netherlands from 2007 to 2010. Neth J Med 2010; 68: 382-7.

4. Landais C, Fenollar F, Thuny F, Raoult D. From acute $Q$ fever to endocarditis: serological follow-up strategy. Clin Infect Dis 2007; 44: 1337-40.

5. Raoult D, Tissot-Dupont H, Foucault C, et al. Q fever 1985-1998. Clinical and epidemiologic features of 1,383 infections. Medicine (Baltimore) 2000; 79: 109-23.

6. Botelho-Nevers $\mathrm{E}$, Fournier $\mathrm{PE}$, Richet $\mathrm{H}$, et al. Coxiella burnetii infection of aortic aneurysms or vascular grafts: report of 30 new cases and evaluation of outcome. Eur J Clin Microbiol Infect Dis 2007; 26: 635-40.

7. Million M, Thuny F, Richet $H$, Raoult D. Long-term outcome of $Q$ fever endocarditis: a 26year personal survey. Lancet Infect Dis 2010; 10: 527-35.

8. Raoult D, Houpikian P, Tissot DH, Riss JM, Arditi-Djiane J, Brouqui P. Treatment of Q fever endocarditis: comparison of 2 regimens containing doxycycline and ofloxacin or hydroxychloroquine. Arch Intern Med 1999; 159: 167-73.

9. Wegdam-Blans MC, Vainas T, van Sambeek MR, et al. Vascular complications of Q-fever infections. Eur J Vasc Endovasc Surg 2012; 42: e19-20.

10. Fenollar F, Fournier PE, Raoult D. Molecular detection of Coxiella burnetii in the sera of patients with Q fever endocarditis or vascular infection. J Clin Microbiol 2004; 42: 491924.

11. Musso D, Raoult D. Coxiella burnetii blood cultures from acute and chronic Q-fever patients. J Clin Microbiol 1995; $33: 3129-32$.

12. Dupont HT, Thirion X, Raoult $D$. Q fever serology: cutoff determination for microimmunofluorescence. Clin Diagn Lab Immunol 1994; 1: 189-96.

13. Li JS, Sexton DJ, Mick N, et al. Proposed modifications to the Duke criteria for the diagnosis of infective endocarditis. Clin Infect Dis 2000; 30: 633-8.

14. Wegdam-Blans MC, Kampschreur LM, Delsing CE, et al. Chronic $Q$ fever: review of the literature and a proposal of new diagnostic criteria. J Infect 2012; 64: 247-59.

15. Kampschreur LM, Oosterheert JJ, Koop AM, et al. Microbiological challenges in the diagnosis of chronic $Q$ fever. Clin Vaccine Immunol 2012; 19: 787-90.

16. Raoult D. Chronic $Q$ fever: Expert opinion versus literature analysis and consensus. J Infect 2012: 65: 102-8. 



\section{Chapter 6}

\section{Serology in chronic $Q$ fever is still surrounded by questionmarks}

Submitted for publication

M.C.A. Wegdam-Blans

H.T. Tjhie

J.M. Korbeeck

M.N. Nabuurs-Franssen

L.M. Kampschreur

T. Sprong

J.A.W. Teijink

M.P.G. Koopmans 


\section{Abstract}

Purpose. Detection of antibodies using immunofluoresence tests (IFAT) is recommended for diagnosis of chronic $Q$ fever, but other commercial antibody assays are also available. Methods. We compared an Enzyme-linked Immunosorbent Assay (ELISA) (Virion/Serion) and a Complement Fixation Test (CFT) (Virion/Serion) for the detection of Coxiella burnetii IgG phase I and IgA phase I in early- and follow-up serum samples from patients with chronic $Q$ fever, diagnosed according to an algorithm that involves IFAT. For this, we tested sera of 49 patients, including 30 proven, 14 probable and five possible chronic $Q$ fever cases. Results. Sensitivity of CFT for diagnosis of chronic $Q$ fever was suboptimal (85\%), as eight patients, including five with chronic $Q$ fever, tested negative at time of diagnosis, whereas IgG phase I antibodies were detected in these five patients by ELISA. Sensitivity of ELISA was higher, although three probable patients were missed. No differences in ELISA IgA phase I detection between proven chronic $Q$ fever and probable were observed; instead possible patient were in majority IgA negative (60\%). Serological examination using ELISA en CFT in follow-up sera from 26 patients on treatment was unsatisfactory. Like IFAT, all kinetic options were possible: decreasing, remaining stable or even increase during time. Conclusions. This study demonstrated that the sensitivity of CFT-based phase I antibody detection is low and therefore not recommended for diagnosis of chronic $Q$ fever. Based on our results, serological follow-up to guide treatment decisions was of limited value. 


\section{Introduction}

Q fever is a zoonosis, caused by the Gram-negative coccobacillus Coxiella burnetii ( $C$. burnetii). There is a large animal reservoir, with goats, sheep and cattle being the most common source of human infections [1-3]. After primary infection, an estimated $1-5 \%$ of patients progress to chronic $Q$ fever, which can become manifest years after initial infection. Endocarditis and infections of aneurysms or vascular prostheses are the most common manifestations [4, 5]. Pre-existent cardiac valvular disease, aortic aneurysm, vascular grafts, immunocompromised state, and pregnancy are reported risk factors for the development of chronic $Q$ fever [6-8]. Since 2007, the southern part of the Netherlands suffered from a major $Q$ fever outbreak, with more than 4000 reported symptomatic cases [9, 10]. As the majority of patients have mild or asymptomatic acute infection, the actual incidence is probably much higher. Due to hygienic measures, vaccination of goats and culling of pregnant goats, the acute $Q$ fever epidemic in the Netherlands has subsided [9]. Nowadays, the brunt of the epidemic in the Netherlands is chronic infections [11]. The diagnosis of chronic $Q$ fever is rather challenging and relies on a combination of clinical symptoms, risk factors, serology, polymerase chain reaction (PCR) and diagnostic imaging. The Dutch consensus group for the diagnosis of chronic $Q$ fever, categorized patients in three levels of certainty based on these factors [12]. According to this guideline, approximately 300 chronic $Q$ fever cases have been registered in the Netherlands. Unfortunately, the sensitivity of $C$. burnetii detection in blood, by PCR or culture, is low. Therefore microbiological evidence is still relying on serology. In the Dutch guideline, an IgG phase I titer of 1:1024, measured with an Indirect Fluorescent Antibody Tests (IFAT), is considered as a threshold, above which, chronic $Q$ fever is considered [12]. Recently, it was shown that there is a strong association between the level of the IFAT IgG phase I titer and PCR-confirmed (proven) chronic $Q$ fever, and it was recommended that IFAT should be used as a screening tool for chronic $Q$ fever [13]. However, immune fluorescence techniques are not used in all laboratories, which may use Enzyme-linked Immunosorbent Assays (ELISA) and Complement Fixation Tests (CFT) instead.

If left untreated, chronic $Q$ fever leads to morbidity and mortality rates up to $60 \%$ [5]. Long-term antibiotic treatment, preferably consisting of hy- 
droxychloroquine and doxycycline, and sometimes aggressive surgery are required in patients with established chronic $Q$ fever [5, 14]. While the guideline can be used for treatment guidance, the duration of treatment is not defined and is done empirically. Currently, based on available literature, treatment is continued until the IgG phase I titer as measured by IFAT has declined fourfold, but with a minimum of 18 months. However, when applying this, we observed examples of treated individuals with sustained clinical response but no detectable effect on IgG phase I titers. Reportedly, compared to patients recovered from acute $Q$ fever, patients with chronic $Q$ fever more often have IgA phase I antibodies, but IgA antibody testing has been abandoned since the introduction of an IFAT IgG cutoff in 1994 [15, 16]. Here, we compared kinetics of antibody responses, measured with IFAT, CFT and ELISA in early and follow-up serum samples from patients with chronic $Q$ fever, in order to find improved serological correlates for patient management.

\section{Materials and methods}

\section{Case definition}

Patients were classified as having proven, probable or possible chronic $Q$ fever according to the recent Dutch guideline [12]. Briefly, the proven classification is used for patients with either positive C. burnetii PCR on blood and/or tissue or with an IFAT phase I IgG titer $\geq 1: 1024$ in combination with a proven (cardio) vascular infection as determined by diagnostic imaging. Probable infection is considered in C. burnetii PCR negative patients with an IFAT phase I IgG titer $\geq 1: 1024$ in combination with known risk factors for chronic $Q$ fever, such as heart valve and vascular prosthesis, but without diagnostic signs of infection by positron emission tomographycomputed tomography (PET-CT) or transoesophageal echocardiography. Possible chronic $Q$ fever is used for patients with IFAT phase I IgG titer $\geq 1: 1024$ without signs or risk factors for chronic $Q$ fever. According to the literature, successful treatment was defined as patients with a fourfold decline in IFAT IgG phase I titer in 18 months or more [17].

\section{Patients}

A total of 49 patients, diagnosed between May 2009 and April 2012 from four different hospitals were included; 32 patients were classified as prov- 
en, 12 as probable and five as possible cases. Antibiotic treatment was started in 32 patients after diagnosis (26 proven and six probable cases). Six proven cases died due to $Q$ fever complications before antibiotic treatment was started. Clinical parameters associated with chronic $Q$ fever, such as fever, abdominal pain and weight loss, as well as treatments (e.g. surgical treatment) were collected from the patient records. Based on these characteristics patients were clustered in three groups: asymptomatic cases, symptomatic cases and symptomatic cases with surgical intervention during antibiotic treatment.

\section{Serum samples}

For comparative analysis of serological test results at time of first sampling $(\mathrm{t}=0)$, serum samples from all 49 patients were available. Ten patients were lost to follow-up due to death or transfer to another hospital, and 13 patients were diagnosed with chronic $Q$ fever recently and therefore followup sera were limited to one year or less. Therefore, a remaining 189 serum samples were analyzed from 26 patients (16 proven and 10 probable cases) treated for at least 18 or 24 months (3-13 samples per patient) to evaluate the antibody kinetics by use of the different methods. All sera were stored at -80 degrees Celsius before serological testing.

\section{IFAT (Focus Diagnostics, Cypress, USA)}

IgG phase I and II, and IgM phase I and II were measured according to the manufacturer's instructions with initial screening of a 1:32 dilution of serum in PBS. Read out was done visually using a fluorescence microscope at 400x magnification. Positive samples were further diluted in this test to establish an endpoint titer. Titers were expressed as the reciprocal of the highest serum dilution showing the diagnostic pattern of fluorescence.

\section{CFT (Virion/Serion, Würzburg, Germany)}

Phase I and II antibodies were measured according to the manufacturer's instructions. After the procedure steps, ready-to-use hemolytic system (Virion/Serion,Germany) was used before visually measuring the titers. Dilutions with $100 \%$ of hemolysis were defined as positive. A positive result was defined as having an endpoint dilution of $\geq 1: 8$. In order to avoid prozone effect all sera were diluted until 1:512. 


\section{ELISA (Virion/Serion, Würzburg, Germany)}

IgG phase I and IgA phase I were processed on a fully automated 4-plate ELISA processing system (DSX). Dilution protocol was used according to the manufacturer's instructions, using 1:100 dilution for IgG as well as for IgA phase I. Data were analyzed according to the Virion/Serion protocol, reporting $\lg G$ and $\operatorname{Ig} A$ phase $\mathrm{I}$. IgG and $\lg A$ phase I were positive whenever the measured absorbance was more than $10 \%$ above the extinction of the cut-off control. Ambiguous results were added to negative results. Extinctions were expressed in OD values.

\section{Data analysis}

Statistical analysis included computations of frequencies and analysis of agreement between ELISA IgG phase I, ELISA IgA phase I, IFAT IgG phase I and CFT phase I. To test significant differences between the three test methods, Chi-square test was used or Fisher's exact test when frequencies had an expectancy of five or less. Spearman's rank correlation was used to investigate the agreement between the IFAT and CFT at $\mathrm{t}=0$. To investigate the kinetics of IgG phase I antibodies, ratios of the titer or OD values at end of treatment (18 or 24 months) and at time of diagnosis $(\mathrm{t}=0)$ were calculated for each method. A ratio above 1 was in accordance with a rise in titer and below one was in accordance with a decline in titer.

\section{Results}

\section{At time of diagnosis}

At $\mathrm{t}=0$, IgG phase I antibodies were more frequently detected with ELISA compared to CFT but this difference was not statistically significant $(93.9 \%$ $(46 / 49)$ and $83.7 \%(41 / 49)$ respectively $(p=0.20)$ (figure 1$)$. This difference was entirely explained by differences in test outcomes for patients with proven $Q$ fever, where IgG phase I antibody ELISA results were in full agreement with the IFAT (100\%; 32/32), whereas five cases were not considered serologically positive by using CFT testing $(83.3 \% ; 27 / 32)$ $(p=0.053)$. Overall, using CFT phase I, eight out of 49 patients $(16.3 \%)$ were undetected. These eight patients had been categorized as proven (5), probable (2) and possible (1) chronic $Q$ fever cases by IFAT based testing combined with criteria as defined in the Dutch consensus guideline. At $t=0$, IgA phase I antibodies were not detected using ELISA in five patients. 
There was no difference in detection between proven and probable cases; in both categories one patient was undetected. Otherwise, three (out of five) possible cases tested negative. Figure 2 shows that comparison of CFT phase I and IFAT IgG phase I at $\mathrm{t}=0$ is good, despite eight negative CFT results (Spearman's rank correlation coefficient of 0.76 .).

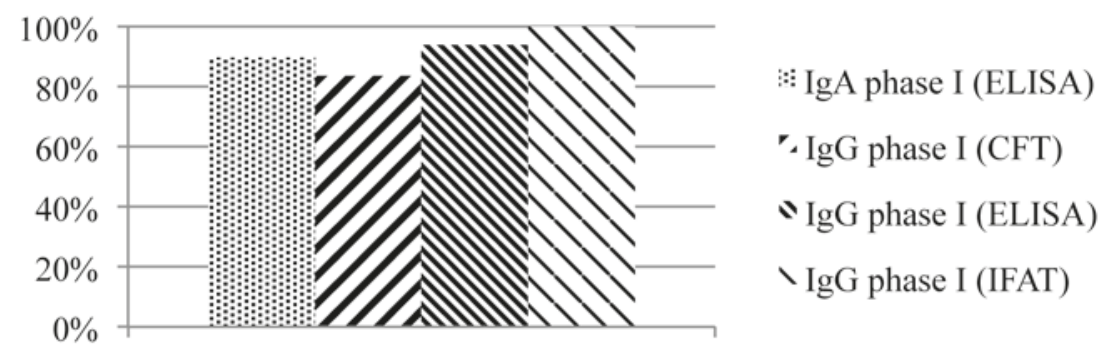

$\%$ positives $(\mathrm{n} / \mathrm{N})$

\begin{tabular}{ll}
\hline ELISA IgA phase I & $89.8(44 / 49)$ \\
CFT IgG phase I & $83.7(41 / 49)$ \\
ELISA IgG phase I & $93.9(46 / 49$ \\
IFAT IgG phase I & $100.0(48 / 48)$ \\
\hline
\end{tabular}

Figure 1. Comparison of serological test.s by percentage of positive results at time of first diagnostic sampling $(\mathrm{t}=0)$.

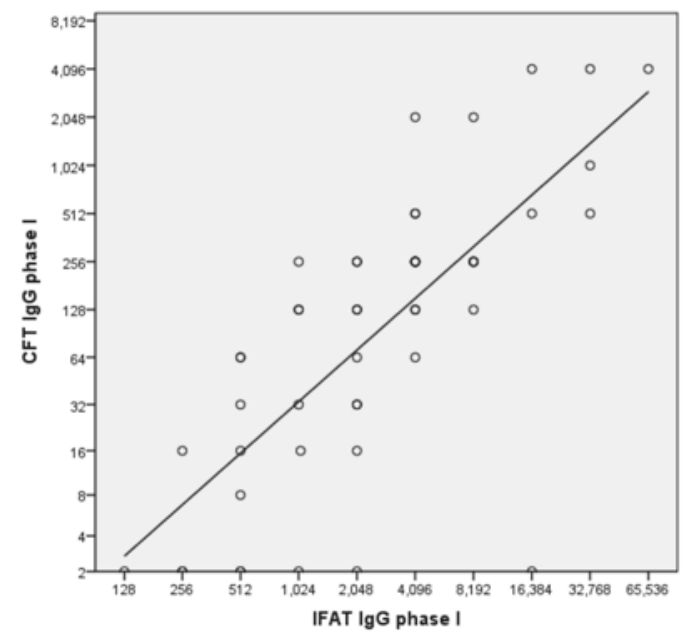

Figure 2. Relation between IgG phase I tests at time of diagnosis $(t=0)$. IFAT $(2 \log )$ and CFT, Spearman's rank correlation $r=0.76$. IFA IgG phase I titer 16 equals IFA IgG phase I titer $<32$; IFA IgG phase I titer 8192 equals IFA IgG phase I titer >4096; CFT phase I titer 2 equals CFT phase I titer <4; CFT phase I titer 1024 equals CFT phase I titer $>512$. 


\section{Follow-up}

The kinetics of IgG phase I antibodies (measured with IFAT, CFT and ELI$\mathrm{SA}$ ) of 26 patients are shown in table 1 and figure 3 . Patients were clustered in three groups: asymptomatic individuals (10), cases with symptoms (8), and cases with surgical intervention during antibiotic treatment (8). In general, it is visible that all kinetic options are possible: decreasing, stable or even increasing antibody levels. Patients were divided in three groups to examine whether the IFAT IgG phase I course better reflects the clinical course of the patients. Kinetics of the IgG phase I antibodies, based on the last and the first serum, is expressed per patients for IFAT, CFT and ELISA (figure 3). In the group of asymptomatic patients, the ratio was $\leq 0.25(=\geq$ fourfold decline) for IFAT in six out of 10 patients. This differs from the symptomatic group with or without surgery: in both groups the minority of patients showed a significant decline using IFAT (ratio $\leq 0.25$ in 3/8 patients in both groups). The numbers are however too small to draw statistical conclusions. When looking for kinetics of antibodies with the other serological methods, a poor correlation was found with the direction of response between measurements with the different assays. With CFT, a ratio $\leq 0.25$ was rarely observed in all three groups, and most of the time (17/23) no significant decline or even an increase was calculated in the ratio. Patients 14, 42 and 12 were excluded, because CFT was negative in the initial sample. With ELISA, calculations differ from CFT and IFAT, because values are not expressed in titers, but in extinctions instead. Therefore, calculation of the ratio was leading to two options: below or above 1. Here again, a poor correlation was observed between the direction of antibody kinetics (increase or decrease) measured with ELISA versus IFAT.

\section{Discussion}

In the present study, based on 49 chronic $Q$ fever patients, we showed that qualitative differences exist in the currently available serological methods for establishing chronic $Q$ fever diagnosis. Also, the use of serological testing as prognostic marker in patients at the end of a treatment period could not be established. 
Table 1. Results of anti IgG phase I test using CFT, IFAT (expressed in titers) and ELISA (expressed in OD value) in 26 patients before and after antibiotic treatment for chronic $Q$ fever of at least 18 months.

\begin{tabular}{|c|c|c|c|c|c|c|c|}
\hline \multirow{2}{*}{$\begin{array}{l}\text { IgG phase I } \\
\text { Category }\end{array}$} & \multirow[b]{2}{*}{ Patient } & \multicolumn{2}{|c|}{ CFT } & \multicolumn{2}{|c|}{ ELISA } & \multicolumn{2}{|c|}{ IFAT } \\
\hline & & $\begin{array}{c}\text { Time of } \\
\text { diagnosis }\end{array}$ & $\begin{array}{l}\text { End of } \\
\text { treatment }\end{array}$ & $\begin{array}{c}\text { Time of } \\
\text { diagnosis }\end{array}$ & $\begin{array}{l}\text { End of } \\
\text { treatment }\end{array}$ & $\begin{array}{c}\text { Time of } \\
\text { diagnosis }\end{array}$ & $\begin{array}{l}\text { End of } \\
\text { treatment }\end{array}$ \\
\hline \multirow[t]{10}{*}{$\begin{array}{l}\text { Non- } \\
\text { symptomatic }\end{array}$} & 1 & NEG & $1: 1,024$ & 0.32 & 1.49 & $1: 128$ & $1: 8,192$ \\
\hline & 2 & $1: 128$ & $1: 64$ & 1.68 & 1.81 & $1: 1,024$ & $1: 512$ \\
\hline & 3 & $1: 512$ & $1: 512$ & 1.64 & 1.52 & $1: 16,384$ & $1: 4,096$ \\
\hline & 4 & $1: 1,024$ & $1: 1,024$ & 1.84 & 1.51 & $1: 32,768$ & $1: 8,192$ \\
\hline & 5 & $1: 16$ & NEG & 1.58 & 0.58 & $1: 256$ & $1: 64$ \\
\hline & 6 & $1: 4,096$ & $1: 256$ & 1.66 & 1.79 & $1: 32,768$ & $1: 4,096$ \\
\hline & 7 & $1: 16$ & $1: 8$ & 1.28 & 1.11 & $1: 1,024$ & $1: 128$ \\
\hline & 8 & $1: 128$ & $1: 64$ & 1.65 & 2.01 & $1: 1,024$ & $2: 1,024$ \\
\hline & 9 & $1: 16$ & $1: 8$ & 1.06 & 1.31 & $1: 2,048$ & $2: 256$ \\
\hline & 10 & $1: 64$ & $1: 32$ & 1.55 & 1.57 & $1: 4,096$ & $1: 2,048$ \\
\hline \multirow[t]{8}{*}{ Symptomatic } & 11 & $1: 256$ & $1: 128$ & 1.19 & 1.87 & $1: 1,024$ & $1: 1,024$ \\
\hline & 12 & $1: 2,048$ & $1: 1,024$ & 1.13 & 1.60 & $1: 4,096$ & $1: 8,192$ \\
\hline & 13 & NEG & NEG & 1.07 & 0.86 & $1: 512$ & $1: 128$ \\
\hline & 14 & $1: 256$ & $1: 256$ & 1.78 & 1.60 & $1: 4,096$ & $1: 2,048$ \\
\hline & 15 & $1: 128$ & $1: 64$ & 1.67 & 1.25 & $1: 2,048$ & $1: 2,048$ \\
\hline & 16 & $1: 64$ & $1: 8$ & 1.54 & 1.27 & $1: 512$ & $1: 128$ \\
\hline & 17 & $1: 512$ & $1: 64$ & 1.59 & $N P^{a}$ & $1: 32,768$ & $1: 2,048$ \\
\hline & 18 & $1: 32$ & $1: 64$ & 1.59 & 1.71 & $1: 512$ & $1: 2,048$ \\
\hline \multirow[t]{8}{*}{$\begin{array}{l}\text { Surgery } \\
\text { performed }\end{array}$} & 19 & $1: 256$ & $1: 32$ & 1.32 & 1.35 & $1: 4,096$ & $1: 1,024$ \\
\hline & 20 & $1: 512$ & $1: 1,024$ & 1.61 & 1.72 & $1: 4,096$ & $1: 4,096$ \\
\hline & 21 & $1: 128$ & $1: 64$ & 1.30 & 1.35 & $1: 4,096$ & $1: 2,048$ \\
\hline & 22 & NEG & $1: 128$ & 0.91 & 1.17 & 1:16,384 & $1: 2,048$ \\
\hline & 23 & $1: 256$ & $1: 128$ & 1.77 & 1.42 & $1: 4,096$ & $1: 1,024$ \\
\hline & 24 & $1: 256$ & $1: 256$ & 1.60 & 1.53 & $1: 2,048$ & $1: 4,096$ \\
\hline & 25 & $1: 2,048$ & $1: 128$ & 1.61 & $N P^{a}$ & $1: 8,192$ & $1: 4,096$ \\
\hline & 26 & $1: 64$ & $1: 64$ & 2.01 & 2.02 & $1: 512$ & $1: 1,024$ \\
\hline
\end{tabular}



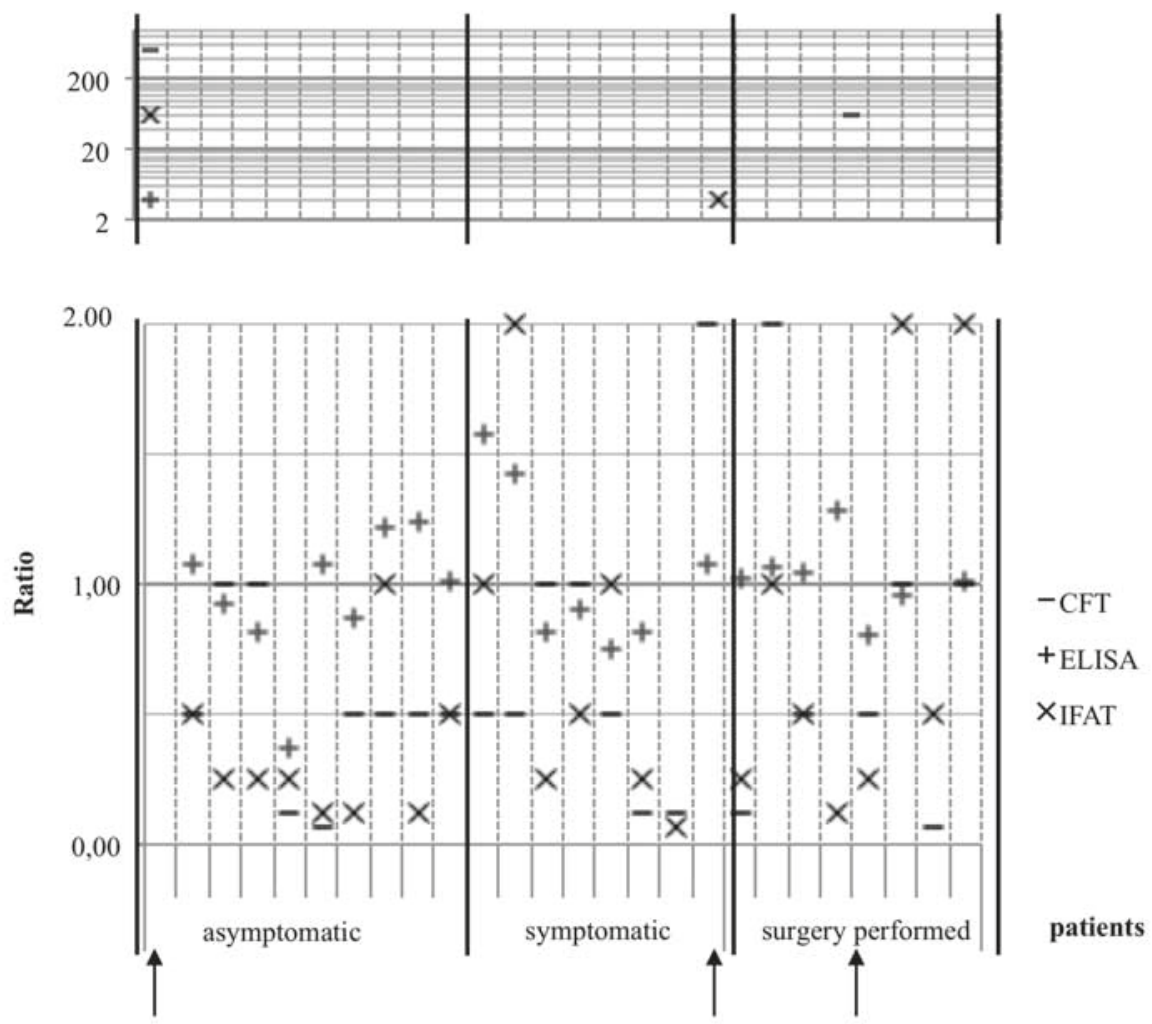

Figure 3. 26 patients have had treatment for at least 18 months. Each bar corresponds with one patient. Ratios of IgG phase I, measured with IFAT, CFT and ELISA, between time of diagnosis and end of treatment; a ratio above 1 is in accordance with a rise, and a ratio below 1 is in accordance with a decline in titer. $\uparrow$ patient with negative CFT value in initial sample.

In the Netherlands, patients are classified as proven, probable or possible chronic $Q$ fever based on serological and PCR results together with clinical symptoms and diagnostic imaging outcomes [12]. Antibiotic treatment is recommended in all proven $Q$ fever patients. Most of the probable patients are treated with antibiotics as well, although this decision is taken after multidisciplinary consultation. Possible $Q$ fever cases are monitored without antibiotic treatment. Cornerstone of serology in chronic $Q$ fever is $\lg G$ phase I measured with IFAT and a level of 1:1024 is agreed as a threshold. In Dutch as well as in other research groups it was demonstrated that high IgG phase I titer and proven chronic $Q$ fever are strongly associated [13, 18]. However, the specificity of the thresholds is low. In different (case) 
reports the clinical significance of high IgG phase I titers was criticized, especially in asymptomatic patients, monitored after acute $Q$ fever patients or in patients identified during screening programs $[19,20]$. Van de Hoek et al. showed that in a follow-up cohort of 686 acute $Q$ fever patients, discrimination between acute and chronic $Q$ fever was difficult as high IFAT phase I antibodies were found in both groups [21]. Although in a much smaller cohort, Min Nan et al. showed the same [20]. Both authors conclude that a high IgG phase I titer in an asymptomatic patient is of limited diagnostic value. In the current study we asked ourselves whether other serological techniques could be used to reduce to this specificity problem.

Different commercial ELISA kits are available for $Q$ fever diagnostics. For diagnosis of acute $Q$ fever, ELISA is proven to be a reliable test [22] and IgG phase II ELISA is routinely used nowadays for this purpose. ELISA is adapted for automation and therefore more suitable for screening purposes than IFAT. Recently, it was demonstrated that the sensitivity of ELISA for high IFAT IgG phase I antibody titers ( $\geq 1: 1024)$ is $100 \%$ [23]. Using the same commercial test we found in our study also $100 \%$ sensitivity for the 30 proven patients in detecting IgG phase I. IgG phase I was undetected with ELISA in 3 patients ( 2 probable and 1 possible). The numbers are too small to draw conclusions, but one could speculate about the possible added value of a negative ELISA IgG phase I in classifying chronic Q fever patients.

A good agreement between the tests regarding the levels of titers was demonstrated between IFAT and CFT, but CFT was less sensitive. Five proven patients were missed in the initial serum. Prozone effect was excluded, as all sera were diluted and tested to 1:1024. Lower sensitivity could be explained by the fact that CFT is not IgG specific.

We tested for IgA phase I antibodies in order to find support for treatment decisions in patients without clinical symptoms but with risk factors for chronic $Q$ fever. However, in our patient series, no significant difference in IgA phase I detection was found between proven and probable patients. In the patients with possible chronic $Q$ fever, the majority (3/5) tested negative. This is in accordance with older studies, where IgA phase I antibodies, measured with IFAT or ELISA, were more often detectable in sera from patients with chronic $Q$ fever compared to acute or past infections [15, 24, 25]. The value of $\lg A$ antibodies in the diagnostics of chronic $Q$ fever has become less prominent in the last two decades, but it could be interesting 
to re-explore its importance more extensively using sera from all Dutch chronic $Q$ fever patients.

We compared the use of different serological assays to monitor treatment effect, as has been recommended for IFAT. The results were quite diverse, showing a poor correlation between clinical response and serological response, and poor correlation between different methods in terms of direction of antibody response. The poor correlation between clinical and serological response may be related by the great diversity in the patient group. There are differences in the organs involved, in co-morbidity and in the time point of diagnosis: some patients are diagnosed with symptomatic disease, others are traced in screening programs. This heterogeneity challenged the interpretation of serological differences between patients. It has been shown that antibodies to $C$. burnetii (IgG as well as $\operatorname{lgM}$ ) are detectable years after the initial infection in fully recovered patients [16, 22], and that follow-up should be extended to 18-24 months [26]. Literature on the use of other assays for serological follow-up is extremely limited, and these were too small to draw conclusions [25, 27].

ELISA could be a good alternative, because there is no observer bias due to the automated way of testing and a half-time is reported to be less than IFAT. However, the ELISA used in our study is designed qualitative, instead of quantitative. Earlier case reports mentioned good serological responses in endocarditis patients, including the former study of Peter et al. $[25,28,29]$. On basis of our results we could not advise the ELSIA IgG phase I assay for follow-up of chronic $Q$ fever patients. However, transforming the assay quantitatively with the use of calibration series could be interesting.

In conclusion, based on the data from this study we can conclude that there is no added value of current alternative tests in the diagnosis of chronic $Q$ fever, compared to IFAT testing. In addition, we were unable to confirm the value of phase I IgG IFAT as a diagnostic marker for clinical response. We conclude that there is a need for improved prognostic and diagnostic markers. 


\section{References}

1. Parker NR, Barralet JH, Bell AM (2006) Q fever. Lancet 367:679-688.

2. Raoult D, Marrie T, Mege J (2005) Natural history and pathophysiology of $Q$ fever. Lancet Infect Dis 5:219-226.

3. Tissot-Dupont H, Raoult D (2008) Q fever. Infect Dis Clin North Am 22:505-514.

4. Botelho-Nevers E, Fournier PE, Richet H, Fenollar F, Lepidi H, Foucault C, et al (2007) Coxiella burnetii infection of aortic aneurysms or vascular grafts: report of 30 new cases and evaluation of outcome. Eur J Clin Microbiol Infect Dis 26:635-640.

5. Million M, Thuny F, Richet H, Raoult D (2010) Long-term outcome of Q fever endocarditis: a 26-year personal survey. Lancet Infect Dis 10:527-535.

6. Fenollar F, Fournier PE, Carrieri MP, Habib G, Messana T, Raoult D (2001) Risks factors and prevention of $Q$ fever endocarditis. Clin Infect Dis 33:312-316.

7. Landais C, Fenollar F, Thuny F, Raoult D (2007) From acute Q fever to endocarditis: serological follow-up strategy. Clin Infect Dis 44:1337-1340.

8. Raoult D, Tissot-Dupont H, Foucault C, Gouvernet J, Fournier PE, Bernit E, et al (2000) $Q$ fever 1985-1998. Clinical and epidemiologic features of 1,383 infections. Medicine (Baltimore) 79:109-123.

9. van der Hoek W, Dijkstra F, Schimmer B, Schneeberger PM, Vellema P, Wijkmans C, et al (2010) Q fever in the Netherlands: an update on the epidemiology and control measures. Euro Surveill doi: pii: 19520.

10. van der Hoek W, Morroy G, Renders NH, Wever PC, Hermans MH, Leenders AC, et al (2012) Epidemic Q fever in humans in the Netherlands. Adv Exp Med Biol 984:329-364.

11. van der Hoek W, Schneeberger PM, Oomen T, Wegdam-Blans MC, Dijkstra F, Notermans DW, et al (2012) Shifting priorities in the aftermath of a $Q$ fever epidemic in 2007 to 2009 in The Netherlands: from acute to chronic infection. Euro Surveill 17:20059.

12. Wegdam-Blans MC, Kampschreur LM, Delsing CE, Bleeker-Rovers CP, Sprong T, van Kasteren ME, et al (2012) Chronic $Q$ fever: review of the literature and a proposal of new diagnostic criteria. J Infect 64:247-259.

13. Kampschreur LM, Oosterheert JJ, Koop AM, Wegdam-Blans MC, Delsing CE, BleekerRovers CP, et al (2012) Microbiological challenges in the diagnosis of chronic $Q$ fever. Clin Vaccine Immunol 19:787-790.

14. Wegdam-Blans MC, Vainas $T$, van Sambeek MR, Cuypers PW, Tjhie HT, van Straten $\mathrm{AH}$, et al (2012) Vascular complications of Q-fever infections. Eur J Vasc Endovasc Surg 42:384-392.

15. Peacock MG, Philip RN, Williams JC, Faulkner RS (1983) Serological evaluation of O fever in humans: enhanced phase I titers of immunoglobulins $G$ and $A$ are diagnostic for Q fever endocarditis. Infect Immun 41:1089-1098.

16. Dupont HT, Thirion X, Raoult D (1994) Q fever serology: cutoff determination for microimmunofluorescence. Clin Diagn Lab Immunol 1:189-196.

17. Raoult D, Levy PY, Harle JR, Etienne J, Massip P, Goldstein F, et al (1990) Chronic Q fever: diagnosis and follow-up. Ann N Y Acad Sci 590:51-60.

18. Frankel D, Richet H, Renvoise A, Raoult D (2011) Q fever in France, 1985-2009. Emerg Infect Dis 17:350-356.

19. de Silva T, Chapman A, Kudesia G, McKendrick M (2006) Ongoing queries: Interpretation of serology in asymptomatic or atypical chronic $Q$ fever. J Infect 52:e113-e116. 
20. Hung MN, Lin LJ, Hou MY, Lin PS, Wang YC, Shu PY, et al (2011) Serologic assessment of the risk of developing chronic $Q$ fever in cohorts of acutely infected individuals. $J$ Infect 62:39-44.

21. van der Hoek W, Versteeg B, Meekelenkamp JC, Renders NH, Leenders AC, WeersPothoff I, et al (2011) Follow-up of 686 patients with acute $Q$ fever and detection of chronic infection. Clin Infect Dis 52:1431-1436.

22. Wegdam-Blans MC, Wielders CC, Meekelenkamp J, Korbeeck JM, Herremans T, Tjhie HT, et al (2012) Evaluation of commonly used serological tests for detection of Coxiella burnetii antibodies in well-defined acute and follow-up sera. Clin Vaccine Immunol 19:1110-1115.

23. van der Hoek W, Wielders CC, Schimmer B, Wegdam-Blans MC, Meekelenkamp J, Zaaijer HL, et al (2012) Detection of phase I IgG antibodies to Coxiella burnetii with EIA as a screening test for blood donations. Eur J Clin Microbiol Infect Dis 31:3207-3209.

24. Dupuis G, Peter O, Luthy R, Nicolet J, Peacock M, Burgdorfer W (1986) Serological diagnosis of $Q$ fever endocarditis. Eur Heart $J$ 1986 7:1062-1066.

25. Peter O, Dupuis G, Bee D, Luthy R, Nicolet J, Burgdorfer W (1988) Enzyme-linked immunosorbent assay for diagnosis of chronic Q fever. J Clin Microbiol 26:1978-1982.

26. Kokkini S, Kofteridis D, Psaroulaki A, Sipsas N, Tsiodras S, Giannitsiotiand E, et al (2009) Q fever endocarditis in Greece: report of five cases. Clin Microbiol Infect 15 Suppl 2:136-137.

27. Worswick D, Marmion BP (1985) Antibody responses in acute and chronic Q fever and in subjects vaccinated against $Q$ fever. J Med Microbiol 19:281-296.

28. Cowley R, Fernandez F, Freemantle W, Rutter D (1992) Enzyme immunoassay for Q fever: comparison with complement fixation and immunofluorescence tests and dot immunoblotting. J Clin Microbiol 30:2451-2455.

29. Field PR, Santiago A, Chan SW, Patel DB, Dickeson D, Mitchell JL, et al (2002) Evaluation of a novel commercial enzyme-linked immunosorbent assay detecting Coxiella burnetii-specific immunoglobulin $\mathrm{G}$ for $\mathrm{Q}$ fever prevaccination screening and diagnosis. J Clin Microbiol 40:3526-3529. 




\section{Chapter 7}

\section{Vascular complications of $Q$ fever infections}

European Journal of Vascular and Endovascular Surgery 2011 Sep;42(3):384-92

M.C.A. Wegdam-Blans

T. Vainas

M.R.H.M. van Sambeek

Ph.W. Cuypers

H.T. Tjhie

A.H.M. van Straten

J.A.W. Teijink 


\section{Abstract}

Introduction. Coxiella burnetii is a strict intracellular pathogen causing $Q$ fever, a worldwide zoonosis with an extensive animal reservoir. Chronic $Q$ fever infections are frequently associated with cardiovascular complications, mainly endocarditis, but also aortic aneurysms and vascular graft infection. We present four cases of chronic $Q$ fever infections and associated vascular complications, and review the literature to identify major symptoms and assess the prevalence, treatment and outcome in these challenging patients. Materials and methods. The demographic and clinical data of 4 patients presenting at our unit were analysed. Pubmed was searched to identify papers describing patients with chronic $Q$ fever associated vascular complications. Results. Combining our own with the published experience, 58 cases (49 male) of chronic $Q$ fever associated vascular complications were identified. The average age of the patients was 64 years (range: 30-83 years). 26 patients had vascular graft infections ( 25 dacron/PTFE, 1 homograft) and 32 had infected aneurysms. The majority of these patients presented with fever $(n=40)$ and/or pain $(n=43)$. Weight loss and fatigue were seen in 25 and 14 patients, respectively. Aneurysm rupture, aortoenteric fistulae and lower limb embolisation were seen in 9, 4 and 4 patients, respectively. Concurrent endocarditis was seen in 2 patients while for 15 cases this information was not available. Patients were treated with antibiotics for an average of 23 months (range 1-54 months). Treatment of infected vascular segments was described in 50 patients. Ten patients were treated conservatively whilst 40 underwent resection of the infected vessel and reconstruction with a graft. Major surgical complications (graft infection, $n=3$; aortoenteric fistula, $n=2$; bleeding, $n=1$; anastomotic leakage, $n=1$; aortic dissection, $n=1$; vertebral osteomyelitis, $n=3$; graft thrombosis, $n=1$; renal failure, $n=2$; pneumonia, $\mathrm{n}=1$ ) were reported in 11 cases $(21 \%)$ and were not specified in 13 . The overall mortality was $24 \%(14 / 58)$. Seven $(18 \%)$ surgically treated patients died. Six of them died within 6 months of surgery and 1 patient at 3 years follow-up. Seven out of ten of the conservatively treated patients died within 3 years of diagnosis. Conclusion. Aneurysms associated with $Q$ fever infections tend to be complicated, requiring challenging surgical corrections, and long term antibiotic treatment. Major complications and mortality rates are significant, especially in conservatively treated patients. 


\section{Introduction}

Coxiella burnetii (C. burnetii) is a strict intracellular pathogen causing $Q$ fever, a worldwide zoonosis with an extensive animal reservoir. Acute and chronic $Q$ fever infections have distinct clinical sequelae and serological profiles, and require different treatment strategies [1]. The acute form of the disease remains asymptomatic in approximately $60 \%$ of cases. The clinical presentation of acute symptomatic cases is mostly non-specific. Symptoms may include flu-like manifestations such as self limiting fever, headache, myalgia, atypical pneumonia and hepatitis. Encephalitis, pericarditis and myocarditis are rare reported manifestations of acute $Q$ fever infections [2]. Chronic $Q$ infections may evolve from acute infections in some hosts with compromised immune systems (leukemia, cancer, HIV infection), cardiac valvulopathy, aortic aneurysms or prosthetic vascular grafts. Indeed, approximately $1.5-11 \%$ of patients with acute $Q$ fever will develop a chronic infection [2-4]. Endocarditis is the main manifestation of chronic $Q$ infection occurring in $60-70 \%$ of all cases, followed by vascular infections, bone infections and chronic hepatitis [5]. It has been estimated that approximately $7 \%$ of chronic $C$. burnetii infections involve patients with aneurysms or vascular graft $[2,6]$.

Outbreaks of $C$. burnetii infections in dairy goat farms occurred from 2007 till 2009 in the Dutch provinces of Noord-Brabant and Gelderland, the catchment area of our vascular service. This was followed by an increased incidence of acute human $Q$ fever cases in the same region during 2007 [7]. Since then, an exponential increase of $Q$ fever cases has been observed. In 2007168 new cases were identified nationwide compared to 2354 new cases in 2009, most of them concentrated in our catchment area. This resulted in an increased prevalence of chronic $C$. burnetii infections and a consequential rise in the prevalence of vascular patients with chronic $Q$ fever [8].

The purpose of this study was to identify important symptoms of patients with vascular infections with Coxiella burnetii in order to give clinicians, especially in endemic area, tools for accurate diagnosis.

\section{Materials and methods}

The demographic, clinical and laboratory data of four patients presenting at our unit with chronic $Q$ fever associated vascular complications were extracted from their electronic patient charts (CS-EZIS, ChipSoft, Amsterdam, 
The Netherlands). The detection of chronic $C$. burnetii infections was based on serology and/or PCR of tissue (if available) or blood (serum or plasma). A commercially available immunofluorescent test was used to detect $C$. burnetii phase I and II IgG antibody titers (Focus, Diagnostics, USA). A positive result both for IgG phase I and II antibodies was defined as having a titer of 1:32 or more. PCR analysis was performed as described by Schneeberger et al. [9]. In accordance with a regional consensus, patients were considered to harbour chronic $C$. burnetii infection when the IgG phase I antibody titer was $>1024$ and equal or greater to the IgG phase II antibody titer [10], or if C. burnetii DNA was detected in blood or tissue by a C. burnetii-specific PCR test [11].

Pubmed, tripdatabase, and sumsearch were searched for papers describing the prevalence, treatment and prognosis of $Q$ fever related vascular complications. The keywords $Q$ fever, Coxiella burnetii, aorta, aneurysm, vascular, and graft were used. Papers that described demographic and clinical treatment and outcome data of chronic $Q$ fever patients with vascular complications were selected. Reference lists of selected papers were hand searched in order to retrieve missing relevant publications. In case of duplicate publications, the most recent report was included in this analysis.

\section{Results}

Within a three month period, four patients with vascular complications and chronic $C$. burnetii infection were treated in our unit.

Case 1. An 83 year old frail woman, suffering from Alzheimer's disease, aortic and mitral valve insufficiency and atrial fibrillation, was admitted with pneumonia. CRP was $39 \mathrm{mg} / \mathrm{L}$ and leucocyte count was $5.5 \times 10^{9} / \mathrm{L}$. An extensive initial microbiological screen, including blood cultures, was negative. However, in the work up for atypical pneumonia, C. burnetii serology was negative for IgM but IgG positive with significantly elevated phase I and II titers (4096 and 4096, respectively) and detection of C. burnetii DNA in plasma (table 1). She was treated with cefuroxime sodium i.v. for community acquired pneumonia and with doxycycline and hydroxychloroquine for chronic $Q$ fever. Abdominal ultrasound revealed the presence of a $5.2 \mathrm{~cm}$ infrarenal aortic aneurysm. Considering her frail condition, the AAA was left untreated and no efforts were undertaken to rule out an endocarditis. She re- 
sponded well to medical therapy and was discharged to a geriatric care facility after 6 weeks of hospitalization. Six months after hospital discharge, the patient remains alive and well.

Table 1. Results of different $C$. burnetii specific diagnostic test in our four cases.

\begin{tabular}{llllll}
\hline & $\begin{array}{l}\text { IgM phase I \& } \\
\text { II titer }\end{array}$ & $\begin{array}{l}\text { IgG phase I } \\
\text { titer }\end{array}$ & $\begin{array}{l}\text { IgG phase II } \\
\text { titer }\end{array}$ & PCR blood & PCR tissue \\
\hline Case 1 & neg & $1: 4096$ & $1: 4096$ & pos & na \\
Case 2 & neg & $1: 4096$ & $1: 4096$ & neg & pos \\
Case 3 & neg & $1: 256$ & $1: 512$ & pos & pos \\
Case 4 & neg & $1: 2048$ & $1: 512$ & neg & pos \\
\hline
\end{tabular}

Abbreviations: na, not available; neg, negative; PCR, polymerase chain reaction; pos, positive.

Case 2. A 68 year old man presented with a growing, non-tender, fluctuating mass in his left flank/paravertebral space. CRP was $24 \mathrm{mg} / \mathrm{L}$ and leucocyte count was $3.5 \times 10^{9} / \mathrm{L}$. An ultrasound scan revealed a large abscess. Since he had undergone emergency endovascular repair for a symptomatic aortoiliac aneurysm just 11 months previously an abdominal CT scan with arterial contrast was obtained. This demonstrated the presence of a paravertebral abscess extending to the left psoas muscle but with no involvement of either the aorta or the vertebral column. No endoleak was present. The abscess was drained. Normal cultures of drained pus were repeatedly negative but $C$. burnetii DNA was detected by PCR in this material. Serology confirmed the presence of chronic $Q$ fever with a $C$. burnetii IgG phase I titer of 4096 and phase II titer of 4096 (table 1). The patient was started on doxycycline and hydroxychloroquine. $Q$ fever endocarditis was ruled out by a negative transthoracic cardiac ultrasound scan and negative plasma C. burnetiispecific PCR. Four months after drainage of the abscess the wound has almost completely healed and the patient remains well continuing antibiotic therapy. Repeat CT angiography revealed a good functioning graft with no signs of an endoleak.

Case 3. A 66 year old woman with a previous tube graft repair of the ascending thoracic aorta for a type A aortic dissection 3 years earlier, was admitted with persisting chest pain, anorexia and weight loss. CRP was 180 $\mathrm{mg} / \mathrm{L}$ and leucocyte count was $13.8 \times 10^{9} / \mathrm{L}$. Blood cultures were negative. A CT scan with arterial contrast revealed a $4.7 \mathrm{~cm}$ pseudoaneurysm at the 
distal suture line. A sternotomy was performed, resecting the distal part of the tubegraft and replacing it with a Dacron Gelweave prosthesis. Cultures of the surgical specimens (native aorta and graft) were positive for Bacteroides fragilis. C. burnetii-specific PCR testing of the native aorta and the resected tube graft were positive. The C. burnetii IgG phase I and II antibody titers were 256 and 512, respectively and IgM antibodies were not detected. C. burnetii DNA was also detected by PCR in plasma (table 1). Valvular vegetations were not seen on transoesophageal cardiac ultrasound. She was started on metronidazole, doxycycline and hydroxychloroquine and has made a further uneventful recovery. The patient was discharged from hospital on the $24^{\text {th }}$ post-operative day. Two months after surgery she remains well and is continuing antibiotic therapy.

Case 4. A 75 year old man presented with a hemodynamic collapse and abdominal pain. His prior medical history included among other things percutaneous coronary intervention and chronic obstructive pulmonary disease. On abdominal CT scan with arterial contrast a ruptured saccular infrarenal aortic aneurysm was seen with massive haemoretroperitoneum. An emergency laparotomy was performed and the aorta was repaired with an inlay Dacron tube graft. Pre-operative blood tests (leucocyte count, C-reactive protein) were not available. Cultures of resected aortic tissue and initial blood cultures were negative. C. burnetii specific PCR test of the aortic tissue was positive but there were no traces of $C$. burnetii DNA in plasma. $C$. burnetii serology was indicative of chronic infection (phase I IgG titer 2048 and phase II IgG 512, table 1). Doxycycline and hydroxychloroquine therapy was initiated. Post-operatively the patient developed kidney failure and two episodes of hemodynamic instability secondary to an Enterococcus faecium bacteremia and a hospital acquired pneumonia. He died on the $39^{\text {th }}$ postoperative day from multiple organ failure.

A literature search identified 17 relevant papers describing cases or case series of chronic vascular Q infections (July 2010) [8, 11-26]. Three papers presented previously published data in combination with new cases. In total, 54 cases of chronic vascular $Q$ fever infections have been reported. An overview of these and our 4 cases is given in table 2. They comprise a heterogeneous group of patients with thoracic and abdominal aortic aneurysms, peripheral arterial aneurysms and aortic or peripheral vascular grafts. The 
majority of patients $(n=43)$ suffered from pain (abdominal, $n=36$; lumbar, $n=7)$ and had fever $(n=40)$. Other non-specific symptoms such as weight loss $(n=25)$ and fatigue $(n=14)$ were common findings. Ten patients had spondylitis. Patients frequently presented with vascular complications. There were 9 vascular ruptures, four aortoenteric fistulas and thromboembolic complications occurred in four cases. Para-aortic abscesses were seen in seven patients (table 3 ). The majority of patients had no signs of endocarditis $(n=41)$, although for 15 cases this information was not mentioned. Two patients had concurrent $Q$ fever endocarditis. One of them developed a stroke due to ultrasonographically detected vegatations of the aortic valve, 2 days after aortic reconstruction. Another patient had aortic valve replacement six months prior to presenting with an infected abdominal aortic aneurysm for which he underwent surgery, but was considered endocarditis negative.

C. burnetii infection of an aneurysm was seen in 32 patients, whilst infection of a vascular graft was seen in 26 patients. The localisation of the infected aneurysm or graft was the infrarenal aorta in 45 patients, the suprarenal aorta in four, the thoracic aorta in six, and the thoracoabdominal aorta in one patient. The splenic, femoral, popliteal and subclavian arteries were all affected once (table 4). Two patients had aneurysms at two sites simultaneously, one had and aortic and (thrombosed) popliteal aneurysm and one patient presented with a splenic artery and a common femoral artery aneurysm.

The diagnosis of $C$. burnetii infection was established by serology in 45 patients. Isolation by culture of $C$. burnetii form the tissue was diagnostic in three patients. Detection of $C$. burnetii DNA in tissue or in blood established the diagnosis in seven and three patients, respectively. 


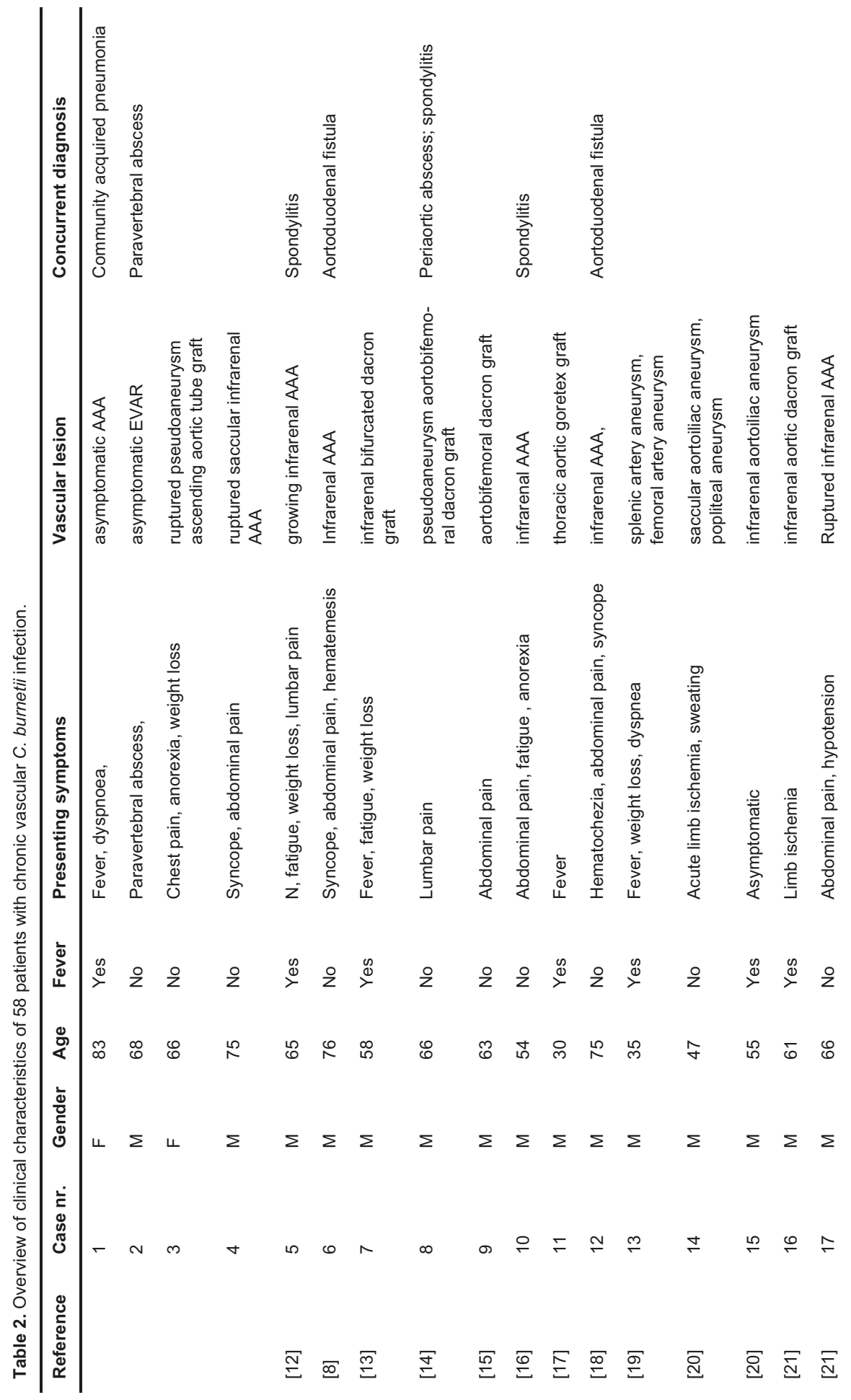




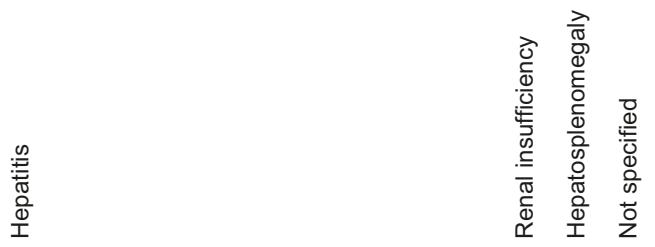

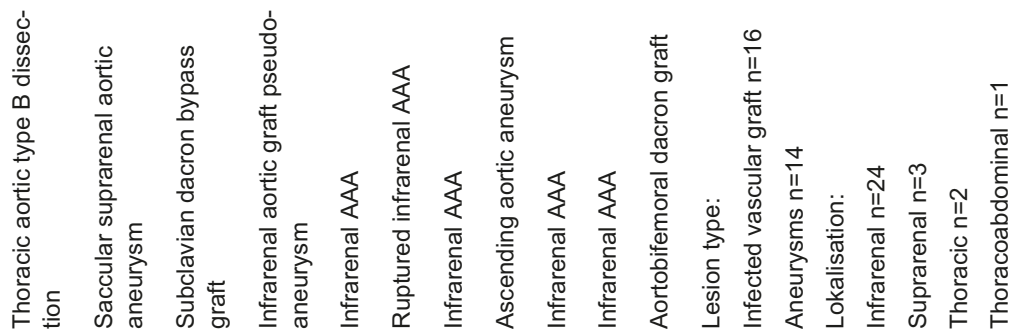

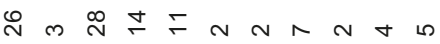

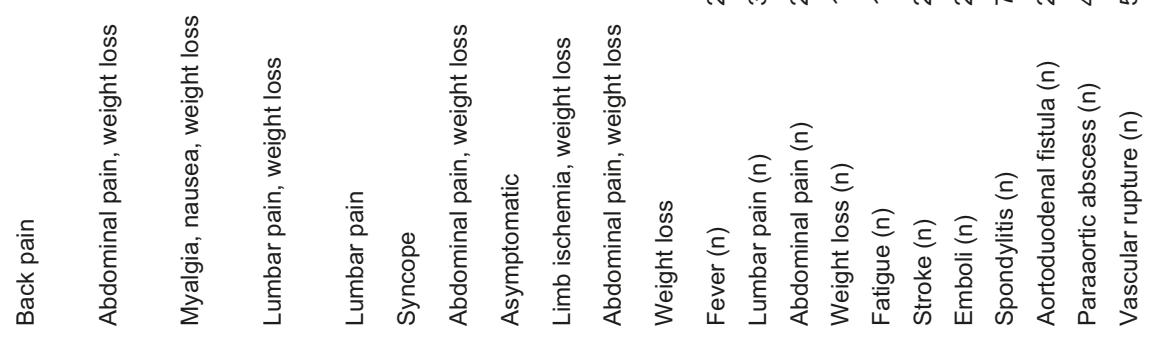

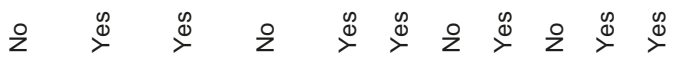

ㄴำ

н

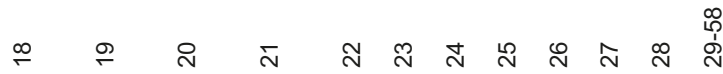

$\underset{i}{F} \equiv$

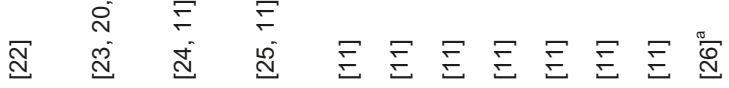




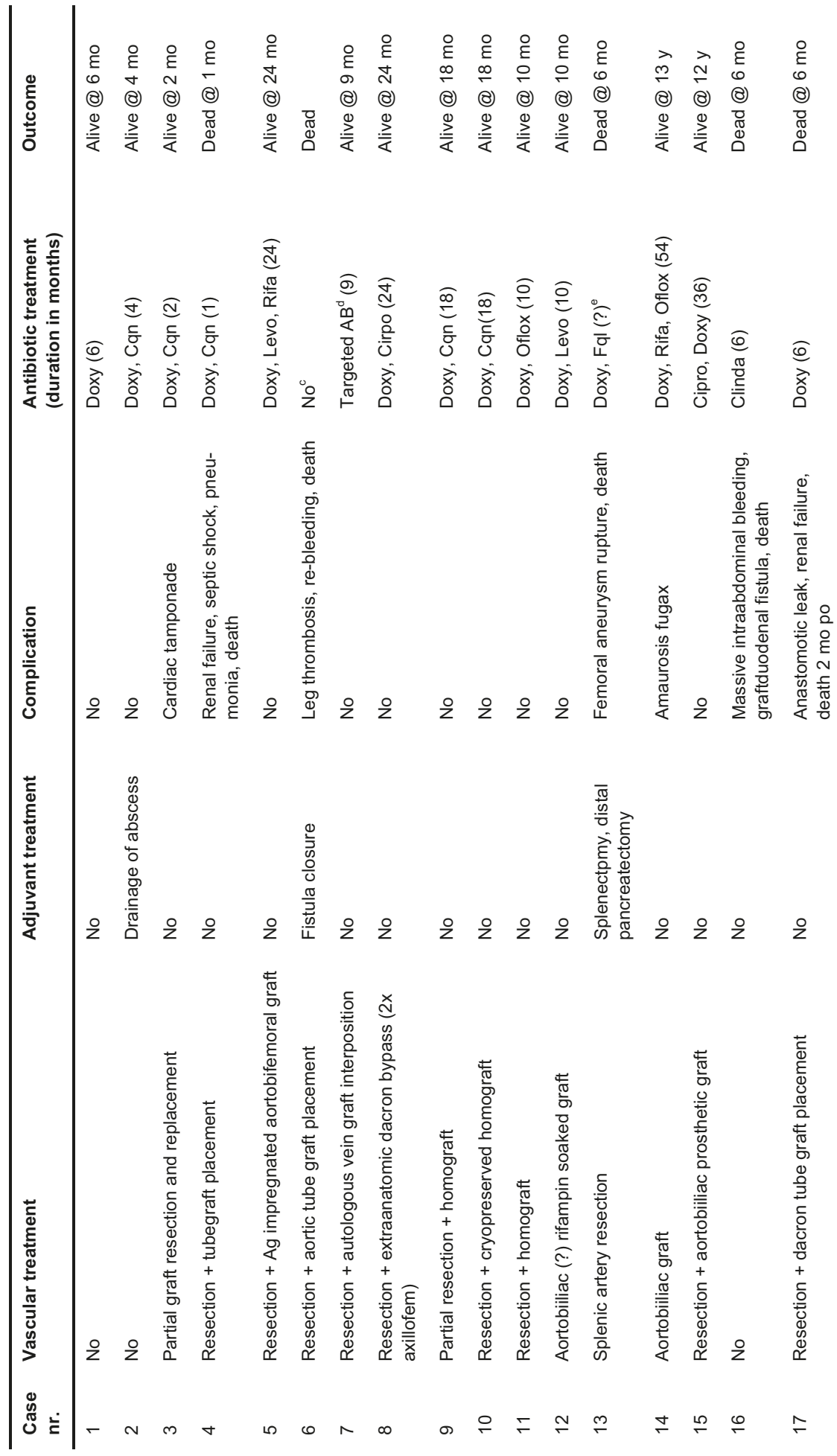




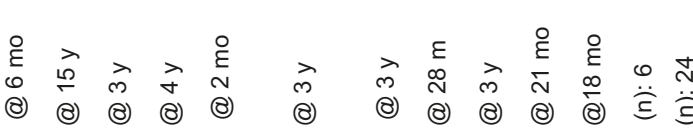

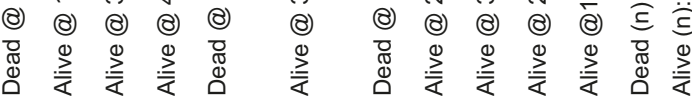

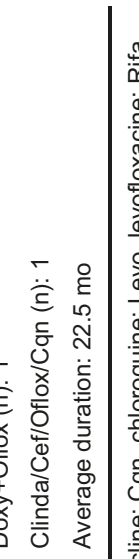

बa

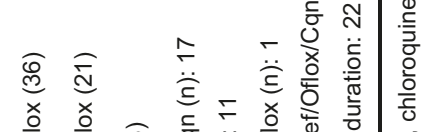

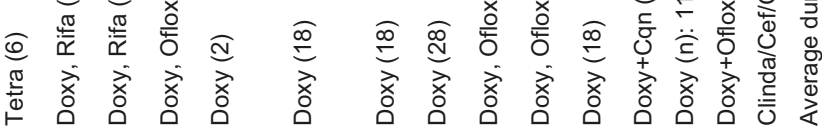
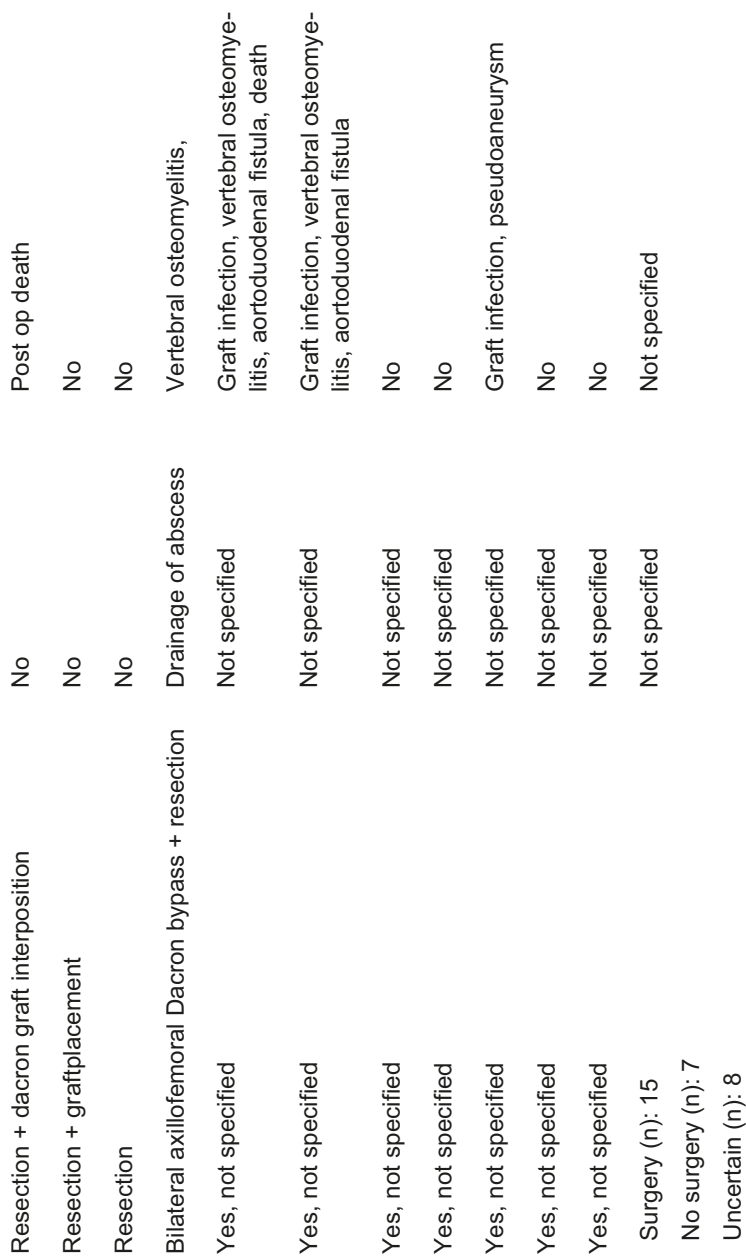

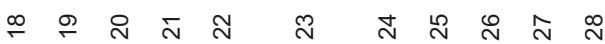

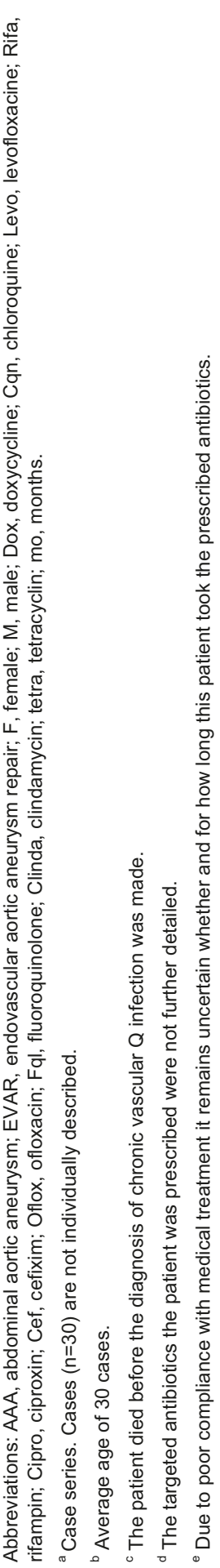


Table 3. Summary of presenting symptoms, vascular treatment and mortality in 58 cases of chronic vascular C. burnetii infection.

\begin{tabular}{ll}
\hline Symptom & $\mathbf{N}(\%)$ \\
\hline Fever & $40(62)$ \\
Lumbar pain & $7(12)$ \\
Abdominal pain & $36(63)$ \\
Weight loss & $25(43)$ \\
Fatigue & $14(24)$ \\
Stroke & $2(3)$ \\
Embolization & $4(7)$ \\
Spondylitis & $10(17)$ \\
Aortoduodenal fistula & $4(7)$ \\
Paraaortic abscess & $7(12)$ \\
Vascular rupture & $9(16)$ \\
Graft pseudoaneurysm & $3(12)^{\mathrm{a}}$ \\
Surgery & $40(69)$ \\
No surgery & $10(17)$ \\
Uncertain treatment & $8(14)$ \\
Major post-operative complication & $11(39)$ \\
Mortality & $14(24)$ \\
\hline 3 pseudoaneurys & \\
\hline
\end{tabular}

a 3 pseudoaneurysms in 26 cases of graft infection.

${ }^{\mathrm{b}}$ Major postoperative complications were not specified in 30 cases.

Table 4. Lesion type and lokalisation in 58 cases of chronic vascular $Q$ fever.

\begin{tabular}{ll}
\hline Lesion lokalisation $^{\mathbf{a}}$ & $\mathbf{N}(\%)$ \\
\hline Infrarenal aorta & $45(78)$ \\
Suprarenal aorta & $4(7)$ \\
Thoracic aorta & $6(10)$ \\
Thoracoabdominal aorta & $1(2)$ \\
Splenic artery & $1(2)$ \\
Femoral artery & $1(2)$ \\
Popliteal artery & $1(2)$ \\
Subclavian artery & $1(2)$ \\
\hline Lesion type & $\mathbf{N}(\%)$ \\
\hline Graft infection & $26(45)$ \\
Aneurysm & $32(55)$ \\
\hline
\end{tabular}

${ }^{\mathrm{a}} 2$ patients had aneurysms at 2 locations. 
Patients were treated with a variety of (a combination of) antibiotics. The majority of patients received doxycycline as monotherapy $(n=18)$ or in combination with hydroxychloroquine $(n=22)$, ofloxacin $(n=5)$, ciprofloxacin $(n=2)$, levofloxacin $(n=1)$, fluoroquinolone $(n=1)$, rifampin $(n=1)$, rifampin and ofloxacin $(n=1)$, or rifampin and levofloxacin $(n=2)$. Tetracycline was given as monotherapy in one patient and clindamycin in another. Finally, one patient was treated with a combination of clindamycin, cefixim, doxycycline and hydroxychloroquine. One patient received 'targeted antibiotics' and one patient who died before the diagnosis could be established did not receive any antibiotics at all. The duration of antibiotic treatment averaged 23 months varying between 0 and 66 months. In general, patients were treated for at least 18 months. Shorter treatment durations were reported for cases with shorter follow up periods or patients who had died.

In the majority of patients but not all, the infected aneurysm or graft was resected. The vascular treatment plan was reported for 50 patients and remained unclear in eight cases. Forty patients were treated surgically and ten patients were treated conservatively. Vascular treatment consisted of resection of the infected aneurysm or graft mainly followed by vascular reconstruction with an homograft or Dacron prosthesis in an anatomic or extraanatomic position. In one patient an infected subclavian-subclavian PTFE cross-over bypass was resected without further reconstruction. In one patient with a splenic and femoral artery aneurysm only the splenic artery aneurysm was resected whilst the femoral aneurysm was left in situ.

Major surgical complications were reported in 11 cases (table 5) and 16 surgical patients recovered apparently without complications. However, the presence or absence of (surgical) complications was not specified in 30 cases.

A total of $14(24 \%)$ fatalities were observed. Among the patients who underwent resection of the infected aneurysm or graft $(n=40)$, seven died. Five patients died within two months of surgery. One patient with a splenic and femoral artery aneurysm died from a rupture of the femoral aneurysm, six months after splenic aneurysm resection. Another patient who had undergone resection of an infected aneurysm died three years later from a ruptured pseudoaneurysm. In the group of patients who were treated conservatively $(n=10)$, one patient died of a massive intra-abdominal haemorrhage six months after being diagnosed with an infected infrarenal aortic prosthesis. The remaining six fatalities occurred in conservatively treated patients within 
3 years of diagnosis. None of the eight patients with unspecified vascular treatment $(n=10)$ have died. If these eight patients with uncertain vascular treatment are not taken into account, the mortality in the conservatively treated group is significantly higher than in the group of patients whose infected aneurysm or graft has been resected $(7 / 10$ vs $7 / 40$, Chi-square 4.98 , $\mathrm{P}=0.025)$.

Two papers assessed the prevalence of chronic $Q$ fever in vascular patients. Fournier and colleagues screened all patients $(n=163)$ with aneurysms or vascular grafts operated in their hospital between January 1995 and December 1996 [11]. In two (1.2\%) patients (one with an AAA and one with a graft) $C$. burnetii was isolated from the vascular specimen. Wever et al. screened 52 patients with AAA in an endemic area and detected $4(7.7 \%)$ patients with a chronic $C$. burnetii infection for whom both serology and PCR testing of blood was positive [8].

Table 5. List of major surgical complications that occurred in 11 patients. $^{\text {a }}$

\begin{tabular}{ll}
\hline Death & $5(19 \%)$ \\
Renal failure & $2(7 \%)$ \\
Pneumonia & $1(4 \%)$ \\
Graft thrombosis & $1(4 \%)$ \\
Anastomotic leak/pseudoaneurym & $3(11 \%)$ \\
Graft infection & $3(11 \%)$ \\
Vertebral ostomyelitis & $3(11 \%)$ \\
Aortoduodenal fistula & $2(7 \%)$ \\
\hline
\end{tabular}

${ }^{\mathrm{a}}$ The presence/absence of surgical complications was reported in only 27 patients.

\section{Discussion}

We reviewed the fate of 58 patients with vascular (extravalvular) chronic $Q$ fever infections. It is a very heterogeneous group of patients with a variety of vascular pathologies, presenting with a range of non-specific, systemic symptoms and not infrequently with acute vascular complications.

The incidence of acute $Q$ fever is low but may vary considerably from 0.15 $0.35 / 100000$ population per year to $7.5-50 / 100000$ population per year in areas where $Q$ fever is endemic [27]. Approximately $1.5-11 \%$ of acute $Q$ fever cases will evolve to chronic $Q$ fever [2-4]. After $Q$ fever endocarditis, vascular C. burnetii infection is the most frequent manifestation of chronic $Q$ 
fever accounting for approximately $8 \%$ of chronic cases $[2,6]$. The low incidence of acute and chronic $Q$ fever may explain the limited number of published reports of patients with chronic vascular C. burnetii infections. However, studies from France have shown that despite the low incidence of acute and chronic $Q$ fever, seroprevalence of specific anti-C. burnetii antibodies was $4 \%$ among blood donors [28] and as high as $30 \%$ in a small rural community [29]. Moreover, Fournier et al. have shown that approximately 1.5\% of patients with aneurysms or vascular grafts have chronic $Q$ fever [11], suggesting that vascular $Q$ fever probably remains underreported. A recent outbreak of $C$. burnetii infections in the Netherlands resulted in an increased incidence of $Q$ fever from 168 cases nationwide in 2007 to 2354 new cases in 2009 [7]. This has resulted in an increase in the number of chronic vascu$\operatorname{lar} Q$ cases in the region. In the period that we treated our four cases, seven more patients with chronic $C$. burnetii infections and abdominal aortic aneurysms have been identified in the same area [8]. The regional incidence of vascular $Q$ infections among aneurysm patients has been estimated to be as high as $8 \%$ [8]. It should be noted that this overview has shown that aneurysms and grafts in virtually every major artery may be affected by chronic $Q$ fever.

This review has shown that chronic $C$. burnetii infection of aneurysms and vascular grafts is associated with significant mortality and morbidity. Treatment of vascular $Q$ infections seems to be based on two premises, i.e. resection of the infected aneurysm or graft and long term antibiotic therapy. Currently no guidelines exist for the antibiotic treatment of vascular $Q$ fever patients. This explains the great variety of (combination of) antibiotics that has been used in the published cases [30-34]. Due to relapse on antibiotic withdrawal combinations of antibiotics has been proposed. Combinations of doxycycline with fluoroquinolone, rifampin, ofloxacin and (hydro)chloroquin have proven clinically effective [27], but even after 3 year treatment intervals, relapse of infection after antibiotic withdrawal remains a problem, although it seems less so for the combination of doxycyclin with chloroquin [35]. The optimum duration of antibiotic therapy for chronic $Q$ endocarditis has not been accurately determined. An acceptable consensus may be administration of antibiotics for at least 18 months or until phase I C. burnetii antibody titers have fallen significantly (four-fold titer decline) [27]. We believe that in the absence of evidence, the same antibiotic regime should be used for chronic vascular $C$. burnetii infections. 
Extrapolation of the lessons learned from the treatment of $Q$ fever endocarditis to vascular $C$. burnetii infections suggests, in the light of the significant relapse rates after antibiotic withdrawal, that surgical excision of the affected vascular segment is an integral part of treatment. Surgical excision of a $C$. burnetii infected aneurysm or graft is technically challenging, not in the least due to the inflammatory reaction at the level of the diseased vascular segment and the frequently occurring complications such as vascular rupture, pseudoaneurysm formation, aorto-enteric fistulae, and paravascular abscesses. Moreover, the chronic infection itself may have weakened the patient prior to undergoing major surgery. This is corroborated by the high rate of major surgical complications and substantial surgical mortality reported in the published cases. Bearing in mind the limitations of the data presented here, it is suggested that surgery seems to confer a survival benefit compared to antibiotic treatment alone in these patients. This, however, may not apply for endovascular aortic reconstructions, since in that case the diseased aorta remains in situ. The second case from our institution presented with an abscess 11 months after endovascular aneurysm repair for a symptomatic AAA. What was interpreted as fluid at the pre-operative CT-scan, may in hindsight be inflammatory tissue in reaction to $Q$-fever aortitis. Since the aneurysm/endograft showed no enhanced activity on a FDG-PET/CT scan we have chosen to treat this patient conservatively with long term antibiotics. Only the future will tell us whether this has been the right decision.

\section{Conclusion}

Chronic vascular C. burnetii infection is a complicated disease with significant mortality and a high complication rate. In patients with vascular pathology and general non-specific symptoms, C. burnetii infection should be investigated. In endemic areas it is wise to screen all patients with vascular pathologies, particularly if the patients present with general symptoms or rare (acute) complications. Considering the small incidence of vascular $C$. burnetii infections, we strongly support the development of collaborative efforts to gather data from patients with vascular $Q$ fever systematically in order to create a sound evidence base that will inform future clinical practice. 


\section{References}

1. Raoult D. Host factors in the severity of Q fever. Ann N Y Acad Sci. 1990;590:33-38.

2. Raoult D, Tissot-Dupont H, Foucault C, Gouvernet J, Fournier PE, Bernit E, Stein A, Nesri M, Harle JR, Weiller PJ. Q fever 1985-1998. Clinical and epidemiologic features of 1,383 infections. Medicine (Baltimore). 2000;79(2):109-123.

3. Fournier PE, Marrie TJ, Raoult D. Diagnosis of Q fever. J Clin Microbiol. 1998;36(7):18231834.

4. Palmer SR, Young SE. Q-fever endocarditis in England and Wales, 1975-81. Lancet. 1982;2(8313):1448-1449.

5. Raoult D, Levy PY, Harle JR, Etienne J, Massip P, Goldstein F, Micoud M, Beytout J, Gallais H, Remy G, et al. Chronic $Q$ fever: diagnosis and follow-up. Ann N Y Acad Sci. 1990;590:51-60.

6. Brouqui P, Dupont HT, Drancourt M, Berland Y, Etienne J, Leport C, Goldstein F, Massip $P$, Micoud M, Bertrand A, et al. Chronic $Q$ fever. Ninety-two cases from France, including 27 cases without endocarditis. Arch Intern Med. 1993;153(5):642-648.

7. Van Steenbergen JE, Morroy G, Groot CA, Ruikes FG, Marcelis JH, Speelman P. [An outbreak of $Q$ fever in The Netherlands--possible link to goats]. Ned Tijdschr Geneeskd. 2007;151(36):1998-2003.

8. Wever PC, Arts $\mathrm{CH}$, Groot CA, Lestrade PJ, Koning $\mathrm{OH}$, Renders $\mathrm{NH}$. Screening op chronische Q-koorts bij symptomatische patiënt met aneurysma of prothese van de aorta. Ned Tijdschr Geneeskd. 2010;154:A2122.

9. Schneeberger PM, Hermans MH, van Hannen EJ, Schellekens JJ, Leenders AC, Wever PC. Real-time PCR with serum samples is indispensable for early diagnosis of acute $Q$ fever. Clin Vaccine Immunol. 2010;17(2):286-290.

10. Croonen H. Q-koorts, ziekte van vele vragen. Een voorzichtig begin van een richtlijn. Medisch Contact. 2010;65(2):52-55.

11. Fournier PE, Casalta JP, Piquet P, Tournigand P, Branchereau A, Raoult D. Coxiella burnetii infection of aneurysms or vascular grafts: report of seven cases and review. Clin Infect Dis. 1998;26(1):116-121.

12. Toral JR, Sainz F, Barberan J, Alguacil R. [Aortic aneurysm and Coxiella burneti]. Enferm Infecc Microbiol Clin. 2008;26(8):535.

13. van Assen S, Houwerzijl EJ, van den Dungen JJ, Koopmans KP. Vascular graft infection due to chronic $Q$ fever diagnosed with fusion positron emission tomography/computed tomography. J Vasc Surg. 2007;46(2):372.

14. O'Donnell ME, Manshani N, McCaughey C, Soong C, Lee B. Coxiella burnetii infection of an aortic graft with multiple vertebral body erosion. J Vasc Surg. 2007;45(2):399-403.

15. Senn L, Franciolli M, Raoult D, Moulin A, Von Segesser L, Calandra T, Greub G. Coxiella burnetii vascular graft infection. BMC Infect Dis. 2005;5:109.

16. Paumier A, Penard J, Maugin E, Enon B, Picquet J. [Infrarenal aortic pseudo-aneurism due to Coxiella burnetii]. J Mal Vasc. 2007;32(4-5):221-224.

17. Georghiou GP, Hirsch R, Vidne BA, Raanani E. Coxiella burnetii infection of an aortic graft: surgical view and a word of caution. Interact Cardiovasc Thorac Surg. 2004;3(2):333-335. 
18. Mejia A, Toursarkissian B, Hagino RT, Myers JG, Sykes MT. Primary aortoduodenal fistula and $Q$ fever: an underrecognized association? Ann Vasc Surg. 2000;14(3):271273.

19. Antonopoulos C, Karagianni M, Galanakis N, Vagianos C. Mycotic splenic artery aneurysm secondary to Coxiella burnetii endocarditis. Ann Vasc Surg. 2010;24(3):416 e413416.

20. Sessa C, Vokrri L, Porcu P, Maurin M, Stahl JP, Magne JL. Abdominal aortic aneurysm and Coxiella burnetii infection: report of three cases and review of the literature. J Vasc Surg. 2005;42(1):153-158.

21. Ellis ME, Smith CC, Moffat MA. Chronic or fatal Q-fever infection: a review of 16 patients seen in North-East Scotland (1967-80). Q J Med. 1983;52(205):54-66.

22. Fergusson RJ, Shaw TR, Kitchin AH, Matthews MB, Inglis JM, Peutherer JF. Subclinical chronic Q fever. Q J Med. 1985;57(222):669-676.

23. Micoud M, Brion JP, Boulard JC, Magne JL, Gratacap B, Stahl JP, Farah I, Raoult D. Infection of aortic aneurysm with Coxiella burnetii. Lancet. 1991;338(8766):584.

24. Raoult D, Piquet P, Gallais H, de Micco C, Drancourt M, Casanova P. Coxiella burnetii infection of a vascular prosthesis. N Engl J Med. 1986;315(21):1358-1359.

25. Piquet $P$, Raoult $D$, Tranier $P$, Mercier $C$. Coxiella burnetii infection of pseudoaneurysm of an aortic bypass graft with contiguous vertebral osteomyelitis. J Vasc Surg. 1994;19(1):165-168.

26. Botelho-Nevers E, Fournier PE, Richet H, Fenollar F, Lepidi H, Foucault C, Branchereau A, Piquet $\mathrm{P}$, Maurin M, Raoult D. Coxiella burnetii infection of aortic aneurysms or vascular grafts: report of 30 new cases and evaluation of outcome. Eur J Clin Microbiol Infect Dis. 2007;26(9):635-640.

27. Maurin M, Raoult D. Q fever. Clin Microbiol Rev. 1999;12(4):518-553.

28. Tissot Dupont H, Raoult D, Brouqui P, Janbon F, Peyramond D, Weiller PJ, Chicheportiche C, Nezri M, Poirier R. Epidemiologic features and clinical presentation of acute $Q$ fever in hospitalized patients: 323 French cases. Am J Med. 1992;93(4):427-434.

29. Bru JP, Stahl JP, Gaillat J, Favier A, Micoud M. Enquete épidémiologique de la fièvre $Q$ dans une commune rurale. Lyon Med. 1983;249:459-461.

30. Turck WP, Matthews MB. Rickettsial endocarditis. Br Med J. 1969;1(5637):185-186.

31. Wilson HG, Neilson GH, Galea EG, Stafford G, O'Brien MF. Q fever endocarditis in Queensland. Circulation. 1976;53(4):680-684.

32. Freeman R, Hodson ME. Q fever endocarditis treated with trimethoprim and sulphamethoxazole. Br Med J. 1972;1(5797):419-420.

33. Subramanya NI, Wright JS, Khan MA. Failure of rifampicin and co-trimoxazole in $Q$ fever endocarditis. Br Med J (Clin Res Ed). 1982;285(6338):343-344.

34. Cacoub P, Wechsler B, Chapelon C, Thibon M, Godeau P. Q-fever endocarditis and treatment with the fluoroquinolones. Arch Intern Med. 1991;151(4):816, 818.

35. Raoult D, Houpikian P, Tissot Dupont H, Riss JM, Arditi-Djiane J, Brouqui P. Treatment of $Q$ fever endocarditis: comparison of 2 regimens containing doxycycline and ofloxacin or hydroxychloroquine. Arch Intern Med. 1999;159(2):167-173.

36. Pedoe HD. Apparent recurrence of $Q$ fever endocarditis following homograft replacement of aortic valve. Br Heart J. 1970;32(4):568-570. 




\section{Chapter 8}

\section{David procedure during a reoperation for ongoing chronic $Q$ fever infection of an ascending aortic prosthesis}

European Journal of Cardio-Thoracic Surgery 2012; 42(1): e19-20

M.C.A. Wegdam-Blans

J.F. ter Woorst

E.G. Klompenhouwer

J.A.W. Teijink 


\section{Abstract}

Chronic $Q$ fever infections, caused by Coxiella burnetii, are associated with cardiovascular complications, mainly endocarditis and vascular (graft) infections. We report a case of a patient with a C. burnetii infected thoracic aorta graft treated initially in a conservative way. However, surgical excision of the infected graft was eventually necessary. This case report highlights the challenges regarding the treatment of patients with chronic vascular $C$. burnetii infections. In the absence of practical guidelines, treatment is tailored to the individual patient. Furthermore, we want to emphasize the need to include chronic $Q$ fever in the differential diagnosis in patients with culture negative aortitis, especially in regions with $Q$ fever epidemics in the recent past. 


\section{Case report}

A 55-year old woman was seen in a peripheral hospital with complaints of weakness, fatigue, dyspnoea and a continuous chest pain for three weeks. Her medical history revealed a supracoronary tube graft repair of the ascending thoracic aorta for a type A aortic dissection three years earlier. Physical examination revealed no abnormalities. C-reactive proteïn (CRP) was $18 \mathrm{mg} / \mathrm{L}$, leukocyte count was $6.3 \times 10^{9} / \mathrm{l}$, erythrocyte sedimentation rate (ESR) was $15 \mathrm{~mm} / \mathrm{h}$. Liver and kidney functions were in normal ranges, besides minor elevations in Gamma-GT and alkaline phosphatase. A computerized tomography (CT) (figure 1A) and a FDG- positron emission tomography-computed tomography (PET/CT) (figure 1B) of the thorax showed a fluid collection around the prosthesis and an increased FDG-uptake of the aortic wall and stentgraft, suspicious for aortitis and eventual prosthetic graft infection. The transthoracic echocardiogram (TTE) showed normal functioning valves without vegetations. Microbiological screening, including repeated blood cultures, was negative. However, in the work-up for culture negative aortitis, Coxiella burnetii (C. burnetii) serology was positive with elevated IgG phase I and II titers (both 1:4096), indicative for a chronic $Q$ fever infection. C. burnetii specific polymerase chain reaction test (PCR) was negative in plasma. Based on the relatively good clinical presentation of the patient, it was initially decided to treat the patient conservatively with antibiotics (doxycycline and hydroxychloroquine). Microbiology parameters, including C. burnetii PCR and serology were checked three monthly. Despite adequate antibiotic serum levels for almost a year, no clinical improvement was documented: the patient still complained of weakness, fatigue and a continuous chest pain. Subsequent CT scans demonstrated augmentation of the fluid mass surrounding the ascending aorta tube graft. IgG levels remained high and after a year $C$. burnetii PCR was positive in the fluid material obtained by a diagnostic puncture as well as in a peripheral blood sample.

Treatment options were again discussed and determined in a multidisciplinary setting. Based on the non-responsiveness to conservative treatment and even progression on CT scan, we decided to treat patient also surgically. The operation was performed using cardiopulmonary bypass through the femoral vein and artery. Immediately after median sternotomy, pus was visible surrounding the prosthesis. After removal of the pus, an aortic clamp was placed and the heart was preserved by crystalloid solution. The infected 
graft was removed and because of a wide aortic root $(40 \mathrm{~mm})$ and a normal functioning aortic valve a David procedure was performed, using a $28-\mathrm{mm}$ Gelweave graft (Vascutek, UK) soaked in rifampicin. The aortic clamp time was 146 minutes and the cardiopulmonary bypass time was 231 minutes. Normothermia was accomplished during the whole procedure. Culture of the infected prosthesis and pus remained negative, but $C$. burnetii DNA was detected by PCR in this material. Moxifloxacine was added to the antibiotic treatment until major side effects urged us to minimize treatment to moxifloxacine only, three months after surgery. Except for drainage of pleural effusion, patient had a good recovery and was discharged after three weeks. Control CT showed a significant decrease of the peri-aortic and retrosternal fluid collection. At six months post-operative, the patient remains alive and well.

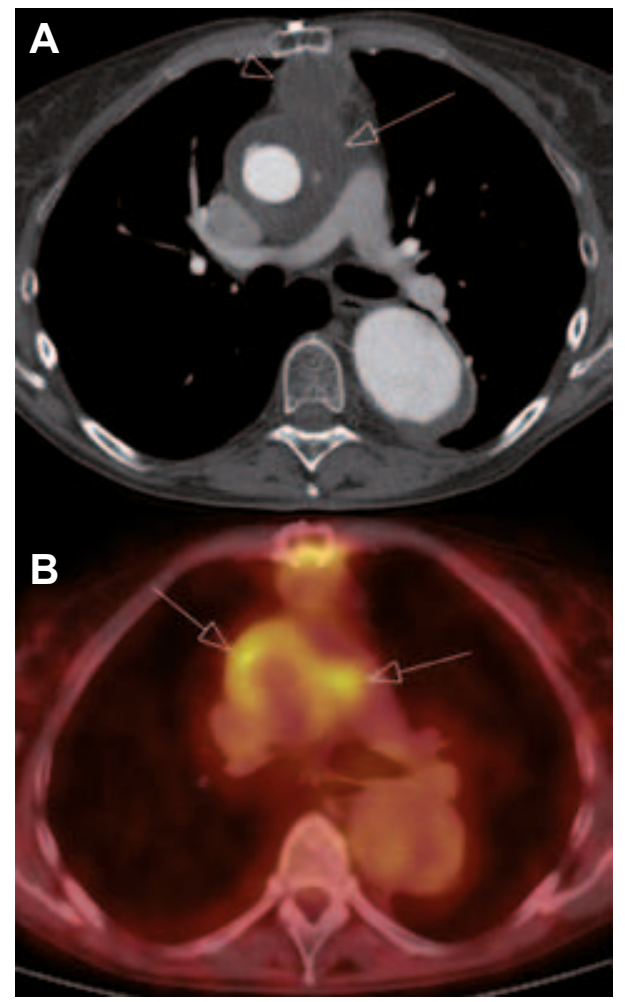

Figure 1. A and B Preoperative CT scan showing a fluid collection around the ascending aorta after tube graft repair (arrow) with expansion to the retrosternal space (arrowhead) (A). Peri-aortitis was suggested clearly by PET-CT showing elevated FDG-uptake of the aortic wall and stand graft of the ascending aorta (arrow) (B). 


\section{Discussion}

Q fever is a zoonosis, caused by the Gram-negative coccobacillus Coxiella burnetii. There is a large animal reservoir, with goats, sheep and cattle being the most common source of human infections [1]. From 2007 till 2009 the Netherlands suffered a major $Q$ fever outbreak, with more than 4000 reported symptomatic cases. As the majority of patients show mild or asymptomatic acute infections, the actual incidence is probably much higher [2]. After primary infection, an estimated 1-5\% of patients progress to chronic $Q$ fever, which can become manifest years after initial infection. Endocarditis and infections of aneurysms or vascular prostheses are the most common manifestations [3]. Pre-existent cardiac valvular disease, an aortic aneurysm, a vascular grafts and/or an immunocompromised state are reported risk factors for the development of chronic $Q$ fever [3]. The diagnosis of chronic $Q$ fever relies mainly on serology and detection of DNA in blood or tissue with PCR. Chronic $Q$ fever is proven when C. burnetii DNA is detected by PCR in blood or tissue, although sensitivity of these techniques in peripheral blood is low [4]. Persisting high levels of antibodies to phase I and phase II antigens are also considered indicative for chronic $Q$ fever [4]. The clinical features of patients with chronic $Q$ fever are non-specific findings such as fatigue, fever, abdominal or chest pain, weight loss, night sweats and hepatosplenomegaly [3]. In the presented patient, only fatigue and chest pain were mentioned. Infection parameters, including CRP, leukocyte count and ESR results were only mildly elevated. This, combined with the fact that most patients with a manifest chronic $Q$ fever infection are unaware of a primary infection with $C$. burnetii, contributes to a significant diagnostic delay. If left untreated, chronic $Q$ fever leads to considerable morbidity and even mortality in up to $60 \%$ [3]. Long-term antibiotic treatment, preferably consisting of hydroxychloroquine and doxycycline is required [3]. However, no comparative trials regarding antibiotic and/or surgical treatment for chronic $Q$ fever are available. In our patient, treatment with both mentioned antibiotics resulted in no clinical improvement. It is plausible that this failure was based on acquired resistance during long lasting antibiotic treatment or as a result of the fact that the infection was situated at a polyester prosthesis in an anatomical position with a low antibiotic penetration possibility. Several surgical problems are encountered in patients with infected grafts. Due to tissue damage, bleeding is of major concern. Therefore, cardiopulmonary bypass is 
needed during the procedure, which can be achieved by cannulation of the patient through the femoral artery and vein before opening the sternum. Tissue around the infected prostheses can be fragile, making it more difficult to fix new graft [5].

In conclusion, we recommend the inclusion of chronic $Q$ fever in the differential diagnosis of patients with culture negative aortitis, especially in regions with $Q$ fever epidemics in the recent past. Due to the deficiency of clinical and microbiological data the criteria for surgical (re-) intervention are not straightforward and should be tailored to the individual patient. 


\section{References}

1. Parker NR, Barralet JH, Bell AM. Q fever. Lancet 2006; 367:679-88.

2. Delsing CE, Kullberg BJ, Bleeker-Rovers CP. Q fever in the Netherlands from 2007 to 2010. Neth J Med 2010; 68:382-7.

3. Botelho-Nevers E, Fournier PE, Richet H, Fenollar F, Lepidi H, Foucault C, Branchereau A, Piquet P, Maurin M, Raoult D. Coxiella burnetii infection of aortic aneurysms or vascular grafts: report of 30 new cases and evaluation of outcome. Eur J Clin Microbiol Infect Dis 2007; 26:635-40.

4. Fenollar F, Fournier PE, Raoult D. Molecular detection of Coxiella burnetii in the sera of patients with Q fever endocarditis or vascular infection. J Clin Microbiol 2004; 42:4919-24.

5. Luciani N, De Geest R, Anselmi A, Glieca F, De Paulis S, Possati G: Results of reoperation on the aortic root and the ascending aorta. Ann Thorac Surg 2011;92:898-903. 



\section{Chapter 9}

\section{Targeted screening as a tool for early detection of chronic $Q$ fever patients after a large outbreak}

European Journal of Clinical Microbiology \& Infectious Diseases 2013; 32(3): 353-9

M.C.A. Wegdam-Blans

R.A. Stokmans

H.T. Tjhie

J.M. Korbeeck

M.P.G. Koopmans

S.M.A.A. Evers

P.H. van der Voort

J.A.W. Teijink 


\section{Abstract}

In the aftermath of the Dutch $Q$ fever outbreak, an increasing number of patients are being diagnosed with chronic $Q$ fever. Most of these patients are unaware of being infected with Coxiella burnetii, the causative agent of $Q$ fever.

To find patients in an earlier, asymptomatic stage, a targeted screening strategy for patients with risk factors for chronic $Q$ fever was started in the southeast region of Noord-Brabant.

In total 763 patients were tested using the IgG phase II IFAT antibody test, of which $52(7 \%)$ patients tested positive. Ten out of 52 patients displayed a chronic $Q$ fever serological profile. All of these 10 patients had a heart valve or (endo-) vascular prosthesis. All except one were asymptomatic. Suggestive signs for chronic infections on PET-CT were demonstrated in $5(50 \%)$ of these patients. Forty-two out of 52 patients with a positive screening test showed a past $Q$ fever serological profile. After a year of follow-up (every three months) none of these patients showed elevation of antibody titers and no new chronic $Q$ fever patients were found in this group.

A targeted screening programme is a useful instrument for detecting patients at risk of developing chronic $Q$ fever. 


\section{Introduction}

Since 2007, the Netherlands has experienced the world's largest outbreak of $Q$ fever, with more than 4000 notified acute $Q$ fever cases. The worst affected areas were the provinces of Noord-Brabant, Gelderland and Limburg [1]. $\mathrm{Q}$ fever is a zoonotic disease caused by Coxiella burnetii (C. burnetii), a small, gram-negative obligate intracellular bacterium, phylogenetically related to Legionellales [2]. Infection in humans results mainly from inhalation of contaminated aerosols [2]. The main source of $C$. burnetii in the Dutch outbreak were dairy goat farms where abortions occurred that released high loads of the bacterium through aborted lambs, placentas and amniotic fluids. The epidemic drastically declined in 2010 after outbreak control measurements, such as culling of goats from $Q$ fever positive goat farms, were put in place by the Dutch Government [3].

Infection with $C$. burnetii may result in acute and chronic infections. The infection remains asymptomatic in up to $50 \%$ of patients [2]. Patients with acute disease fever usually present with fever, mild flu-like symptoms and pneumonia two to six weeks after exposure [2]. Because these symptoms are non-specific, under-reporting is quite substantial, especially at the beginning of an outbreak.

An estimated $1-5 \%$ of patients progress to chronic $Q$ fever, usually insidiously, months or even years after primary infection [2, 4, 5]. Patients often present with non-specific symptoms such as low-grade fever, night sweats and weight loss. Endocarditis is described as the predominant manifestation of chronic $Q$ fever, followed by vascular wall infection, chronic infection in pregnancy, osteomyelitis and chronic hepatitis [4-7]. Both asymptomatic and symptomatic acute $Q$ fever cases are at risk of developing chronic infection. Known risk factors for developing chronic infection are valvular heart disease, including heart valve prostheses, vascular prostheses and aneurysmatic diseases of the aorta [4-7]. Unlike acute $Q$ fever, chronic $Q$ fever is a potentially lethal disease. Morbidity and mortality are related to the time of diagnosis and the start of combination antibiotic therapy and surgical intervention [7].

In the southeast region of Noord-Brabant, we were confronted with 17 patients with chronic $Q$ fever in a short period of time (July 2009-August 2010). The majority of cases (12) had vascular wall infections, either mycotic aneurysms or infected vascular prostheses. Two patients were diagnosed with 
endocarditis and three patients with leukaemia, had chronic $Q$ fever with unknown focus. Five patients were admitted to the Intensive Care Unit because of hemodynamic instability in an emergency setting and died shortly after. The primary cause of death was major bleeding due to $Q$ fever induced arterio-bronchial fistulae in two cases, and multi-organ failure due to septic shock in three cases. Notably, only two of all 17 chronic $Q$ fever cases detected in the region had previously been diagnosed with acute $Q$ fever. A detailed description of the clinical manifestation in the first four patients was already published [8].

The high number of serious chronic infections led us to question whether a targeted screening strategy (TSS) would detect these cases earlier, possibly with the benefit of a better prognosis. Therefore, we started a TSS programme, as suggested by the ECDC for patients with risk factors for chronic $Q$ fever [9]. In this article we described the results of this programme.

\section{Methods}

\section{Risk definition}

The target patient group consisted of patients known with abdominal aortic aneurysms, a (endo-) vascular prosthesis of the aorta or heart valve(s) prosthesis and living in one of the five communities with the highest incidence of acute $Q$ fever in the southeast region of Noord-Brabant.

\section{Procedure}

All four hospitals (including one cardiothoracic and endovascular referral centre) in our region participated. Target patients were asked to participate in the screening programme using two different strategies. Firstly, retrospective lists of patients were created by searching into the hospital registration files using the Diagnosis and Treatment Combination (DBC) codes from January 2000-August 2010. Patient lists were checked for doubles and crosschecked with hospital registration files to filter out all deceased people. The selected patients received an invitation letter to participate in this programme between September and November 2010. Secondly, between September 2010 and September 2011, patients who had been recently diagnosed with the aforementioned patient characteristics and presenting at regular outpatient clinic visits, were asked by their attending physicians to participate in the programme. Those who agreed to participate received a 
package containing an information letter explaining chronic $Q$ fever and the purpose of the screening programme, an informed consent form, and laboratory forms. Patients were asked to return the written consent form and to give blood (EDTA and serum).

\section{$Q$ fever definitions}

Patients were classified as negative for $Q$ fever infection ( $\lg \mathrm{I}$ I and $\|<1: 32$ ), past $Q$ fever infection (IgG phase $\| \geq 1: 32$ and IgG phase I <1:1024 and negative PCR) or chronic $Q$ fever infection (IgG I $\geq 1: 1024$ and/or positive $P C R)$, based on the recently published national guideline for the diagnosis of chronic $Q$ fever [10].

\section{Q fever assay}

Sera were tested for $C$. burnetii antibodies using an Indirect Fluorescent Antibody Test (IFAT) IgG phase I/phase II test (Focus Diagnostics, Cypress, USA). C. burnetii IgG phase I and II antibodies were measured directly from serum using a single dilution of 1:32. Positive samples were further diluted in this test to quantify an end-point titer. Antibody positive sera were subjected to $C$. burnetii PCR, using EDTA blood.

Seronegative patients were asked to return for follow-up serological testing for $C$. burnetii antibodies once within six months. Patients with a serological result indicative of past infection were asked to return for follow-up every three months for one year. Patients with serological results suggestive of chronic infection were contacted by their specialist for immediate consultation and treatment by the infectious disease specialist (figure 1).

Clinical, laboratory diagnostic imaging results of chronic $Q$ fever patients. Clinical symptoms fitting a chronic infection, C-reactive protein (CRP), erythrocyte sedimentation rate (ESR) leucocyte count and results of transthoracic echocardiography (TTE) and/or positron emission tomographycomputed tomography (PET-CT) were recorded during the first visit by the infectious disease specialist.

\section{Treatment of chronic $Q$ fever patients}

Chronic $Q$ fever patients were treated according to the Dutch guidelines [10]. Whenever antibiotics were indicated, a combination of doxycycline and hydroxychloroquine was the regiment of choice [11]. 


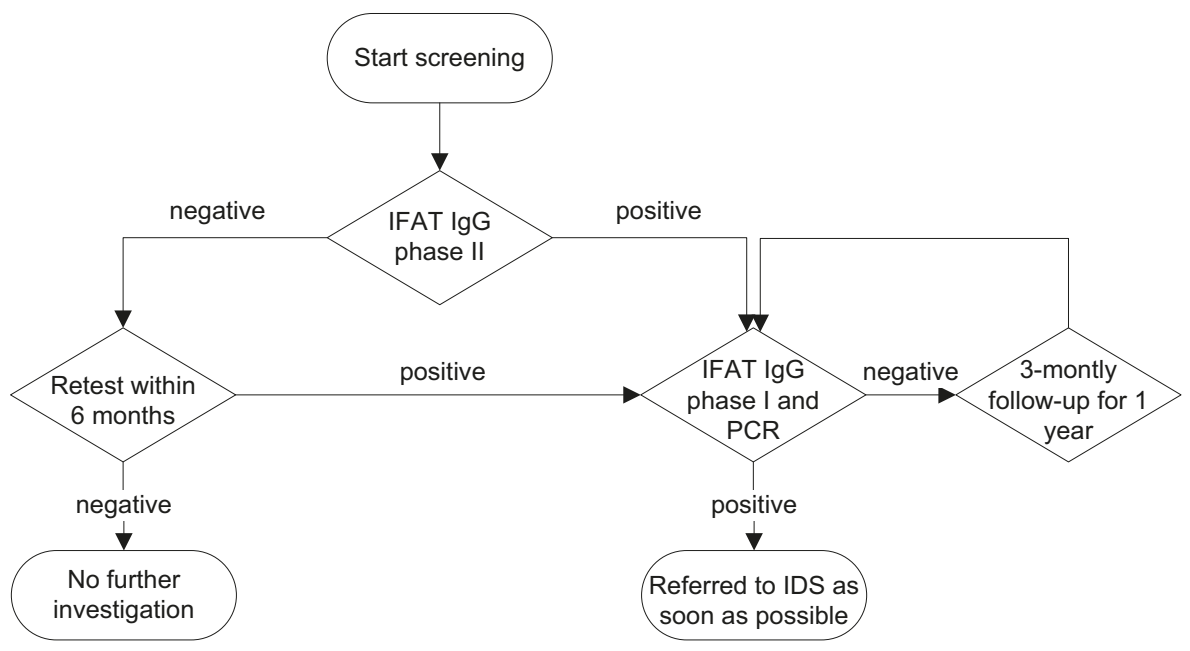

Figure 1. Flow chart of the case-finding program, the parallelograms and rounded rectangles depict decisions and endpoints of the screening phase respectively. IFAT IgG phase II is positive $\geq 1: 32$, IFAT IgG phase I is positive for chronic $Q$ fever $\geq 1: 1024$.

\section{Statistical analysis}

Data from the laboratory information system (MOLIS, vision4health, Barchon, Belgium) and patients' characteristics were collected using a datacentric application built on Microsoft technologies. Statistical analysis was performed using 1-tailed binominal tests to test for significant differences in patients with and without chronic $Q$ fever concerning sex and prosthesis.

\section{Ethical considerations}

Ethical Committees of Catharina Hospital Eindhoven, Elkerliek Hospital Helmond, St. Anna Hospital Geldrop and Máxima Medical Centre Veldhoven/Eindhoven (all four participating centres) approved the screening programme. Written informed consent was collected from all participating patients.

\section{Results}

Strategy 1: Based on the selection criteria, 1,032 patients were invited, of which $526(51 \%)$ submitted at least one blood sample. All non-responders received a reminder, resulting in 54 more patient samples being submitted. Strategy 2: At regular outpatient clinic visits, 234 additional patients were recognised as "at risk patients", and all of these agreed to participate. In total, 763 patients were available for analysis, of which $75 \%(n=569)$ were 
male. Most of the patients were between 55 and 85, with a mean age of 72 years. Almost half of the group $(48 \% ; n=363)$ had a previous cardiothoracic intervention (44 patients with a thoracic aortic reconstruction and 319 patients with a valve prosthesis). Abdominal aortic aneurysms were present in $52 \%(n=400)$ of the cases, treated conservatively in $30 \%(n=227)$, with an endovascular intervention in $13 \%(n=101)$ or with an open reconstruction in $9 \%(n=72)$ (table 1).

Q fever serology was positive in $7 \%(n=52)$, including three patients who tested positive upon repeat sampling. Of these patients, $81 \%(n=42 / 52)$ had serology results suggesting past infection. Ten patients (19\%) had $Q$ fever serology indicating chronic infection, and C. burnetii DNA was detected in a blood sample of two of these. All patients were found with strategy 1 . The main characteristics of the patients with chronic $Q$ fever serology are mentioned in table 2.

All patients but one were male, this 9:1 ratio of male and female patients does not significantly differ from the population tested $(p=0.234)$. Prosthesis appeared to be a significant risk factor. All patients had a (endo-) vascular prosthesis of the aorta or heart valve(s) prosthesis and no conservatively treated aneurysms $(p=0.029)$. Interestingly, only one patient reported symptoms consistent with a chronic infection. Also laboratory parameters for infection were low. Diagnostic imaging, using PET-CT was positive for infection of the prosthesis in five patients $(50 \%, 5 / 10)$. In six patients with heart valve(s) prosthesis, no valve vegetations were seen using trans-thoracic echocardiography (TEE). Seven patients were started with specific anti $Q$ fever antibiotic treatment. The patients detected with chronic $Q$ fever were followed during an average period of 16.4 months (varying with a standard deviation of 2.7 months) until July 2012 and none of the patients had died.

Of the patients with a past infection, $88 \%(n=37 / 42)$ were followed at regular 3-monthly intervals for a year. A serological exacerbation or a positive $C$. burnetii PCR was found in none of the sera. 
Table 1. Risk factors of the 763 patients tested, by age group and gender.

\begin{tabular}{|c|c|c|c|c|c|c|c|}
\hline \multicolumn{2}{|c|}{ Riskfactors $^{a}$} & \multicolumn{2}{|c|}{$\begin{array}{l}\text { Cardiothoracic } \\
\text { intervention }\end{array}$} & \multicolumn{3}{|c|}{ Abdominal aorta aneurysm } & \multirow[b]{2}{*}{ Total } \\
\hline $\begin{array}{l}\text { Gen- } \\
\text { der }\end{array}$ & $\begin{array}{l}\text { Age } \pm \\
5 \text { years }\end{array}$ & $\begin{array}{l}\text { Thoracic } \\
\text { aorta } \\
\text { prosthesis }^{\text {a }}\end{array}$ & $\begin{array}{l}\text { Valve } \\
\text { prosthesis }\end{array}$ & $\begin{array}{l}\text { Conserva- } \\
\text { tive treat- } \\
\text { ment }\end{array}$ & $\begin{array}{l}\text { Endovas- } \\
\text { cular } \\
\text { prosthesis }\end{array}$ & $\begin{array}{l}\text { Open } \\
\text { recon- } \\
\text { struction }\end{array}$ & \\
\hline \multirow[t]{9}{*}{ Male } & 10 & & & 1 & & & 1 \\
\hline & 30 & & 1 & & & & 1 \\
\hline & 40 & 2 & 3 & 2 & & & 7 \\
\hline & 50 & 4 & 10 & & & & 14 \\
\hline & 60 & 10 & 31 & 24 & 10 & 8 & 83 \\
\hline & 70 & 8 & 82 & 70 & 35 & 27 & 220 \\
\hline & 80 & 9 & 61 & 75 & 44 & 24 & 215 \\
\hline & 90 & 1 & 8 & 13 & 4 & 1 & 27 \\
\hline & 100 & & & 1 & & & 1 \\
\hline Male & Total & 34 & 196 & 186 & 93 & 60 & 569 \\
\hline \multirow{7}{*}{$\begin{array}{l}\text { Fema- } \\
\text { le }\end{array}$} & 30 & & 2 & & & & 2 \\
\hline & 40 & & 3 & & & & 3 \\
\hline & 50 & 1 & 9 & 1 & & & 11 \\
\hline & 60 & 1 & 18 & 6 & 1 & 1 & 28 \\
\hline & 70 & 4 & 35 & 16 & 1 & 6 & 62 \\
\hline & 80 & 3 & 48 & 17 & 4 & 5 & 76 \\
\hline & 90 & 1 & 8 & 1 & 2 & & 12 \\
\hline $\begin{array}{l}\text { Fema- } \\
\text { le }\end{array}$ & Total & 10 & 123 & 41 & 8 & 12 & 194 \\
\hline Total & & 44 & 319 & 227 & 101 & 72 & 763 \\
\hline
\end{tabular}

a Patients having a combined thoracic aorta and valve prosthesis ("Bentall procedure", $n=15$ ) were categorised as thoracic aorta prosthesis.

\section{Discussion}

A targeted screening programme for chronic Q-fever after an acute $Q$ fever outbreak appears to be a good approach to detecting asymptomatic chronic $Q$ fever patients at an early stage. We hypothesize that the outcome of these patients, in terms of mortality and morbidity, improves based on the premises of adequate therapeutic regimen and regular examination by an infectious diseases specialist. 
Table 2. Main characteristics of the 10 patients with chronic $Q$ fever serology.

\begin{tabular}{|c|c|c|c|}
\hline Patient characteristics & & $n / N$ & (Range) \\
\hline \multirow[t]{2}{*}{ Gender } & Male & $9 / 10$ & \\
\hline & Female & $1 / 10$ & \\
\hline \multirow[t]{4}{*}{ Risk } & Vascular prosthesis & $4 / 10$ & \\
\hline & Heart valve prosthesis & $3 / 10$ & \\
\hline & Combined prosthesis & $3 / 10$ & \\
\hline & Aorta aneurysm & $0 / 10$ & \\
\hline \multirow[t]{2}{*}{ Serology and PCR } & IgG phase I positive $(\geq 1: 1,024)$ & $10 / 10$ & $(1: 1,024-$ \\
\hline & PCR positive in blood & $2 / 10$ & \\
\hline \multirow[t]{2}{*}{ Positive diagnostic imaginga } & TEE & $0 / 6$ & \\
\hline & PET-CT & $5 / 10$ & \\
\hline \multirow{5}{*}{$\begin{array}{l}\text { Clinical and laboratory characte- } \\
\text { ristics }\end{array}$} & Symptoms $^{\mathrm{b}}$ & $1 / 10$ & \\
\hline & CRP & $10 / 10$ & $(<6-24)$ \\
\hline & ESR & $10 / 10$ & $(2-26)$ \\
\hline & Leukocyte count & $10 / 10$ & $(4.7-11.5)$ \\
\hline & Antibiotic treatment & $7 / 10$ & \\
\hline
\end{tabular}

${ }^{a}$ Trans-oesophageal echocardiogram (TEE) was performed in six patients. Positron emission tomography-computed tomography (PET-CT) was performed in all patients.

${ }^{b}$ Symptoms of chronic $Q$ fever (fever, night sweats, weight loss, abdominal pain and/or shiffers) were recorded during the first intake of the infectious disease specialist.

The $Q$ fever outbreak in the Netherlands was exceptionally large, with at least 4000 cases reported from 2007 to 2009 . The acute $Q$ fever epidemic in the Netherlands has now subsided due to improved hygienic measures on farms, culling of pregnant goats and vaccination of goats [1, 3]. Nevertheless, the $Q$ fever outbreak was followed by a rising number of chronic $Q$ fever patients [3]. Due to the insidious course of the disease, it is possible that chronic $Q$ fever becomes manifest many months, or even years after the primary (acute $Q$ fever) infection $[2,4,5]$. Therefore it was expected that months, even years after the outbreak, patients with chronic $Q$ fever were likely to continue to present.

The main manifestation of chronic $Q$ fever found in the literature appeared to be endocarditis [4-8]. Prosthetic valves as well as native valve disorders are indicated as risk factor for chronic $Q$ fever [5-7]. Vascular wall infection, including that of aneurysms and vascular prostheses is the second most common manifestation of chronic $Q$ fever, although the percentages known 
from the literature are far less than endocarditis: $60-80 \%$ and $10-20 \%$ for endocarditis and vascular wall infection, respectively [5-7]. In the Netherlands, these incidences appeared to be the opposite [12]. This was also the case in our symptomatic patient group, in which we found vascular wall manifestations sixfold more than endocarditis. The reason for this discrepancy is not known. It is possible that the course of chronic $Q$ fever endocarditis is slower than a vascular wall infection and the group of endocarditis patients still has to emerge. Another possible explanation is previous under-reporting of vascular wall disease due to unfamiliarity.

The success of TSS depends on the definition of the risk population. Theoretically, all inhabitants of our region were at risk of developing chronic $Q$ fever. However, screening of all inhabitants within a short period of time would not have been practical and would have proved to be relatively expensive. For these reasons we narrowed our target population down to those patients who were assumed to have the highest risk for developing chronic $Q$ fever. We focused our search on patients with existing or previously treated abdominal and/or thoracic aortic aneurysms, vascular prostheses, or heart valve prostheses. These conditions were highly present in the 17 symptomatic chronic $Q$ fever cases that were found in the participating four hospitals. Although native valve disorders are mentioned as being at risk for chronic $Q$ fever, we did not include this group in our risk definition, based on the heterogeneity of valve disorders as well as earlier Dutch reports about the limited contribution of native valve disorders in chronic $Q$ fever [13].

Immune compromised disorders were also not included. Although we detected three symptomatic chronic patients with leukaemia before starting the programme, we decided not to extend the case definition to those with this underlying disease. It would be interesting to check for chronic $Q$ fever in immune compromised hosts as well, however we found ourselves confronted with a very heterogeneous patient group who were not supported by any data other than case reports to estimate the risk. We presumed that patients with these vascular and cardiac conditions, who had not visited one of the hospitals in the past ten years, had either died or had moved out of the region. Therefore we limited our search to all records from January 2000. Schimmer et al. showed that the distance of residence from infected goat farms and location of human cases and number of infected farms was epi- 
demiologically linked [14]. Therefore, we used the geographic area around five communities with the highest incidence of acute $Q$ fever.

From the 52 seropositive patients in our study, $75 \%$ was male and only one of the 10 chronic $Q$ fever patients was female. It is known from the literature that the male gender is a risk factor for symptomatic diseases. Female hormones may confer a protective role [4]. Of the seropositive tested patients $19 \%(n=10)$ had a serological profile consistent with chronic $Q$ fever. In the literature, the risk of developing chronic disease after initial infection is up to $40 \%$ in patients with risk factors [5-7]. Although a substantial part of our seropositive patients had a chronic $Q$ fever profile, it is lower than mentioned in the literature. All chronic $Q$ fever patients detected in our study were known to have a (endo-) vascular prosthesis of the aorta or heart valve(s) prosthesis. Although the presence of an aortic aneurysm (in surveillance) is a known risk factor for chronic $Q$ fever, we did not find any patient with this. This could (partly) be explained by the fact that infected aneurysms (mycotic aneurysms) rapidly lead to symptoms and therefore will not be picked up in a TSS programme in an outpatient clinic setting. None of the performed transthoracic echocardiography (TEE) was indicative for endocarditis. It is known from the literature that the sensitivity of echocardiography in $Q$ fever endocarditis is very low, due to the formation of none or very small vegetations on the valves [7]. None of the patients, except one, reported symptoms consistent with chronic infection. Also, the laboratory parameters for infection were low. In contrast, PET-CT was positive for infection of the prosthesis in five patients. This finding corresponds with the idea that chronic $Q$ fever has mostly an insidious course and that advanced tissue infection precedes clinical manifestation. Also we did not find any patients with reactivation. It was suggested in the literature that $C$. burnetii can reach a dormant stage and can reactivate years after infection [15]. That would mean that patients with a past serological profile will develop a chronic serological profile, months or years after acute infection. We hypothesize that chronic infection is a slow, continuous process that follows acute symptomatic infection. That would mean that patients have already developed a chronic serological profile very soon after an acute infection. Longer follow-up of patients at risk with a past serological profile are necessary to prove this. If it is true, than sequel serological testing is not necessary for patients at risk, once a serological test fits past infection. 
If we apply the principles of screening composed by Wilson and Junger [16] on our screening program, we meet all criteria except that no consensus is established whether such programs should be introduced at a national level. Also the case definition is still a matter of debate. In the Netherlands, chronic $Q$ fever patients are included in a national database. Comparing the outcome (mortality and morbidity) of patients detected by screening with the outcome of those presenting with symptomatic chronic $Q$ fever should give the final answer whether screening after a $Q$ fever outbreak is effective or not. Another omission in our study was the lack of cost analysis. Due to the design of our study, cost analysis was not possible. Cost analysis should be a component when the effectiveness of a screening programme is evaluated. However, one can imagine that the cost of serology testing per patient is negligible, compared to the costs of treatment of symptomatic chronic $Q$ fever patients. Costs for this screening programme were based on administrative activities, paper and postal charges, analytical hands-on time and the cost price of the serological test, which was performed in a single dilution screening procedure. The total cost was approximately $€ 10$ per patient. In our region, $10 \%$ of the blood donors were found positive for $C$. burnetii IgG phase II antibodies (unpublished data, source: The National Blood Bank, Stichting Sanquin, Amsterdam, The Netherlands). According to several studies, there is a risk of up to $40 \%$ to progress from acute to chronic $Q$ fever in patients with risk factors [2-5]. Therefore, the calculated cost to detect 1 case of chronic $Q$ fever would be $€ 250$. The expenditures in health costs for a symptomatic chronic $Q$ fever patients of course depends on different issues such as the length of admission, ICU stay, operative procedures, materials used and laboratory and imaging tests carried out. Based on our first 10 consecutive symptomatic patients admitted to the participating hospitals before the programme was started, the average cost of treating a symptomatic chronic $Q$ fever patient was $€ 21,900$ per patient. Costs were strongly influenced by the necessity for surgical procedures: the average costs of six patients who had gone through a surgical procedure, was about $€ 36,500$ per patient.

The definition of chronic $Q$ fever was guided by the serological results of the screening. This is the major limitation of our study. At the moment we do not know how to treat patients who show high IgG phase I titers and no clinical or radiological signs of chronic infection. In the recently published Dutch guidelines, this group is classified as possible chronic $Q$ fever patients [10]. 
The classification might need adjustment when more prospective clinical data become available.

In summary, symptomatic chronic $Q$ fever is a severe disease with high morbidity and mortality. In our study, we detected 10 patients at an earlier stage of the disease. A real cost analysis was not included in this study, but we assume that serological screenings assays are low compared to the costs involved with the care for symptomatic chronic $Q$ fever patients. These facts, together with the moral obligation to provide the best medical care for chronic $Q$ fever patients in a post-epidemic period, makes a targeted screening programme as presented here advisable. Although our data are preliminary, we hypothesize that acute infection passes into chronic infection as a continuous process. 


\section{References}

1. Delsing CE, Kulberg BJ, and Bleeker-Rovers CP (2010) Q fever in the Netherlands from 2007 to 2010. Neth J Med 68:382-7.

2. Parker NR, Barralet JH, and Bell AM (2006) Q fever. Lancet 367:679-88.

3. van der Hoek W, Schneeberger PM, Oomen T, et al (2012) Shifting priorities in the aftermath of a Q fever epidemic in 2007 to 2009 in the Netherlands: from acute to chronic infection. Euro Surveill 17:20059.

4. Raoult D, Marrie T, and Mege J (2005) Natural history and pathophysiology of Q fever. Lancet Infect Dis 5:219-26.

5. Raoult D, Tissot-Dupont H, Foucault C, et al (2000) Q fever 1985-1998. Clinical and epidemiologic features of 1,383 infections. Medicine 79:109-23.

6. Botelho-Nevers E, Fournier PE, Richet $\mathrm{H}$, et al (2007) Coxiella burnetii infection of aortic aneurysms or vascular grafts: report of 30 new cases and evaluation of outcome. Eur $\mathrm{J}$ Clin Microbiol Infect Dis 26:635-40.

7. Million M, Thuny F, Richet $\mathrm{H}$, et al (2010) Long-term outcome of $\mathrm{Q}$ fever endocarditis: a 26-year personal survey. Lancet Infect Dis 10:527-35.

8. Wegdam-Blans MC, Vainas T, van Sambeek MR, et al (2011) Vascular complications of $Q$ fever infections. Eur J Vasc Endovasc Surg 42:384-92.

9. Forland F, Jansen A, de Carvalho Gomes H, et al (2010). Risk assessment on Q fever. European Centre for Disease Prevention and Control (ECDC) Stockholm 2010. doi: 10.2900/28860. Available from: http://ecdc.europa.eu/en/publications/Publications/1005_TER_Risk_Assessment_Qfever. pdf

10. Wegdam-Blans MC, Kampschreur LM, Delsing CE, et al (2012) Chronic Q fever: review of the literature and a proposal of new diagnostic criteria. J Infect 64:247-59.

11. Raoult D, Houpikian P, Tissot Dupont $H$, et al (1999) Treatment of $Q$ fever endocarditis: comparison of 2 regimens containing doxycycline and ofloxacin or hydroxychloroquine. Arch Intern Med 159:167-73.

12. Kampschreur LM, Dekker S, Hagenaars JC, et al (2012) Identification of risk factors for chronic Q fever, the Netherlands. Emerg Infect Dis 18:563-70.

13. Limonard GJ, Nabuurs-Franssen MH, Weers-Pothoff G, et al (2010) One-year follow-up of patients of the ongoing Dutch $Q$ fever outbreak: clinical, serological and echocardiographic findings. Infection 38:471-7.

14. Schimmer B, Ter Schegget R, Wegdam M, et al (2010) The use of a geographic information system to identify a dairy goat farm as the most likely source of an urban $Q$ fever outbreak. BMC Infect Dis 10:69.

15. Harris RJ, Storm PA, Lloyd A, et al (2000) Long-term persistence of Coxiella burnetii in the host after primary $Q$ fever. Epidemiol Infect 124:543-9.

16. Wilson JMG, Jungner G. Principles and practice of screening for disease. World Health Organization public health papers. 1968;34. Geneva: World Health Organization; 1968. Available from: http://whqlibdoc.who.int/php/WHO_PHP_34.pdf. 




\section{Chapter 10}

Summary and general discussion 
Between 2007 and 2009, the Netherlands experienced the world's largest outbreak of $Q$ fever, with more than 4,000 notified cases of acute $Q$ fever [15] The worst affected areas included the provinces of Noord-Brabant, Gelderland and Limburg. Dairy goat farms have been identified as the main source of the $C$. burnetii infection in this outbreak, with large amounts of the bacterium being released during a number of abortions through infected aborted lambs, placentas and amniotic fluids [6-8]. An annual seasonal peak was clearly observed; most patients were identified in spring, during the lambing season [3-5, 8]. Research established that the environment was highly contaminated with aerosols containing $C$. burnetii, which thus could spread kilometers from its initial source [9, 10]. The epidemic drastically declined in 2010 after outbreak control measurements, such as the culling of goats from $Q$ fever positive goat farms, were put in place by the Dutch government [8]. The magnitude and the duration of the Dutch outbreak were of an unprecedented scale: for three years, a population of three provinces had been exposed to vast amounts of $C$. burnetii. Therefore, the Dutch situation cannot be compared to earlier small outbreaks or small case series of $Q$ fever that have been reported in the literature [11-13]. In this new epidemiological situation, new observations were made regarding diagnostic and clinical performances. Laboratories in the affected areas were challenged by the great number of requests, and, at the onset of the outbreak, uniformity in diagnostic testing as well as in test result interpretation was lacking. Various Dutch medical microbiology laboratories worked on the basis of independently developed flowcharts, which were put to the test while meeting the increased demand for diagnostics. Therefore, the Dutch National Institute for Public Health and the Environment (RIVM), in conjunction with the Netherlands Society for Medical Microbiology (NVMM), established a ' $Q$ fever consensus group', consisting of medical microbiologists and infectious disease specialists, who had gained experience with diagnosis and treatment of $Q$ fever. The consensus group, initially started for acute $Q$ fever diagnostics, was founded in 2010. Since then, several reports and guidelines have been produced (see insert), and several specific data gaps have been identified. Studies addressing some of these data gaps are described in chapters 2, 3, 4 and 6. 
Output of the 'Dutch $Q$ fever consensus group'

Guideline for laboratory diagnosis of acute $Q$ fever

Guideline for diagnosis of chronic $Q$ fever

Establishment of a periodic multidisciplinary meeting of treatment of chronic $Q$ fever patients

Advise on strategies for a screening test for blood and tissue donation References [14-16]

\section{Diagnosis of acute $Q$ fever}

The interpretation of test results is not always straightforward, due to the use of different serological test methods and the background prevalence of the disease. Various different serological tests for $Q$ fever are used in the Netherlands, including Indirect Fluorescent Antibody Tests (IFAT), Enzyme-linked Immunosorbent Assays (ELISA) and Complement Fixation Tests (CFT). Therefore, uniformity in the diagnostics and interpretation of $Q$ fever, in support of outbreak control, was the most important aim for the consensus group. Before the formation of this group, the different tests had not been thoroughly evaluated. In 2010, RIVM conducted a multicenter quality assessment for $Q$ fever serology showing that qualitative values between laboratories using the same methods were within close range, and all three methods correctly identified acute $Q$ fever patients [17]. However, these observations were based on a small sample size. More research was required to establish a greater degree of clarity and uniformity in the use and interpretation of acute $Q$ fever diagnostics. Therefore, we conducted a study, described in chapter 2, comparing ELISA and CFT for the detection of $C$. burnetii IgG phase I, IgG phase II and IgM phase II in acute and follow-up sera [18]. For this, we collected a unique array of sera, drawn in the phase of acute illness and continuing on a three-monthly basis (for the duration of a year), from 126 patients with acute $Q$ fever diagnosed by positive $C$. burnetii PCR in blood. Based on IgG phase II antibody detection in paired samples from 62 patients, all three tests performed almost equally well in confirming acute $Q$ fever cases (100\% IFAT, 95.2\% ELISA and 96.8\% CFT). The limitation of this study was that we lacked sequential samples of the first weeks after infection, as second samples were drawn from patients only after three 
months. Therefore we cannot derive too many conclusions on the tests in this early phase after initial infection. We showed that IgM phase II is already detectable in a proportion of acute sera collected at time of the onset of the disease. In these sera, IgM was positive in $31.8 \%$ and $19.7 \%$ of the cases with IFAT and ELISA respectively. Unfortunately, however, IgM was detectable up to at least twelve months (our maximum observation period) after the initial diagnosis in most patients, especially when measured with IFAT. Therefore, definitive serological evidence of acute $Q$ fever cannot be based on IgM in a single serum sample in post-epidemic or endemic areas where infections are common. If a confirmation of the diagnosis is required, a seroconversion or a significant (fourfold) titer rise for IgG phase II must be conducted to confirm the serologic diagnosis in paired serum samples in epidemic or high-endemic areas. Our results showed that the tests are comparable in this respect. Significant differences were demonstrated in follow-up sera in IgG phase I and phase II antibody detection. The decline of IgG phase II antibody titers was significantly slower with IFAT as compared to ELISA and CFT, which makes IFAT the preferable method in studies in which high sensitivity is essential, such as vaccination strategies. Vaccination can give severe side effects in patients with past infection, therefore it is mandatory to exclude a past infection. This study demonstrated that the three serological tests are equally effective in serum paired diagnosis of acute $Q$ fever, yet IFAT detects all measured antibodies (IgM phase I, IgG phase I and IgG phase II) with significantly greater sensitivity in both early and late samples. The choice of test depends on the indication. Laboratories can choose their diagnostic tests with the results derived from our study.

$Q$ fever is a notifiable disease. According to Dutch national law, all notifications of laboratories and physicians should be investigated by an infectious disease specialist of the Dutch Municipal Health Services (MHS). Such an investigation is mainly aimed at tracking down (new) sources, which is the principal reason for $Q$ fever notification. To monitor the evolution of the epidemic, it was agreed with the MHS to impose a mandatory reporting duty for both confirmed as well as suspected (probable or possible) cases of acute $Q$ fever [19]. Furthermore, it was arranged that an evaluation of the notification rules would be required to assess in hindsight whether this strategy had indeed been beneficial in epidemic control. In chapter 3, we described this evaluation by comparing the percentages of notified cases to the National 
Case Register (NCR) during and after the outbreak. Based on the laboratory results, acute $Q$ fever can be either diagnosed as: 1. confirmed (PCR positive or significant increase in IgG phase II), 2. probable (single serum detection of IgG and IgM phase II) or 3. possible (single serum detection of IgM phase II). In the Netherlands, the notification system has been working as a two-tiered system: after an initial notification of a confirmed, probable or possible acute $Q$ fever patient by the laboratory to the MHS, the MHS infectious disease specialist would review whether the case also met the clinical criteria. If so, the notification was reconfirmed and reported to the NCR. We hypothesized that the number of reconfirmed cases would decrease in the post-epidemic area due to detection of antibodies from past (asymptomatic) infection, as also pointed out in chapter 2 . In a retrospective study of 523 patients notified to the MHS in 2009, 2010 and 2011, we indeed demonstrated a significant decrease of confirmed acute $Q$ fever cases. For laboratory confirmed cases, the proportion of reconfirmed and reported cases to the NCR decreased by $50 \%$ in the years 2010 and 2011. This decline was even steeper for cases that were diagnosed as 'probable' and 'possible $Q$ fever' in laboratory tests; in 2010 and 2011, almost none of the notifications reached the NCR. The notification of past infections is not contributing the identification of new sources of infection, but will merely give GGDcolleagues needless amounts of work. Based on our results, we recommend downsizing the notification criteria after an epidemic to only confirmed acute $Q$ fever cases.

\section{Diagnosis of chronic $Q$ fever}

After the acute $Q$ fever epidemic in the Netherlands had subsided, a growing number of patients with chronic infections were identified in the affected areas. Chronic $Q$ fever usually progresses insidiously, months or even years after the primary infection [9, 20-22]. Patients often present with non-specific symptoms such as low-grade fever, night sweats and weight loss [20, 22, 23]. Severe complications, such as aortic wall rupture can occur, especially in patients with diagnostic delay [23]. Unlike acute $Q$ fever, chronic $Q$ fever is a potentially lethal disease. For clinicians, like cardiologists, vascular surgeons and infectious disease specialists, chronic $Q$ fever was a new clinical entity with an increasing number of patients in a short time. There was an urgent need for uniformity in the diagnosis of chronic $Q$ fever. In order to 
give those clinicians the best possible support for treating patients for chronic $Q$ fever infections, the Dutch $Q$ fever consensus group decided to establish a guideline. Therefore we performed a systematic review of the available literature on the clinical aspects and diagnostic tools for chronic $Q$ fever. As there were no randomized trials available, meta-analysis of the selected articles was not possible. Nonetheless, the major outcomes of these studies have been described in chapter 4. Based on these data we proposed to classify chronic $Q$ fever in three categories, combining clinical, radiological and microbiological factors [16]. Based on the available level of evidence, we distinguished between proven chronic $Q$ fever at the highest level, probably chronic $Q$ fever in the middle, and possible chronic $Q$ fever at the lower end of the spectrum. Proven chronic $Q$ fever patients are classified as positive C. burnetii PCR (or culture) in blood or tissue, or patients with phase I $\lg \mathrm{g} \geq 1: 800$ or phase I IgG $\geq 1024$ (depending on in-house IFA technique or commercial IFA technique respectively), in combination with definite endocarditis according to the revised Duke criteria, or evidence of infection of aneurysm or vascular graft on CT, PET-CT, duplex ultrasound or MRI. Probable chronic $Q$ fever is diagnosed in those patients with phase I IgG $\geq 1: 800$ or phase I IgG $\geq 1: 1024$ who have established risk factors for chronic $Q$ fever, show echocardiographic abnormalities that do not meet the revised Duke criteria, and/or probable rare manifestations of $Q$ fever (e.g. hepatitis, osteomyelitis) or signs of systemic inflammation. Possible chronic $Q$ fever, finally, is diagnosed in patients with solely a phase I IgG $\geq 1: 800$ or phase I IgG $\geq 1: 1024$, without any of the manifestations mentioned in the categories proven and probable. Long-term antibiotic treatment, preferably consisting of hydroxychloroquine and doxycycline, must always be initiated in case of proven chronic $Q$ fever. In patients with probable chronic $Q$ fever, the indication for antibiotic treatment must be discussed in a multidisciplinary team, consisting of clinicians and microbiologists with experience in chronic $\mathrm{Q}$ fever. In our opinion, patients with possible chronic $Q$ fever are not indicated for antibiotic treatment, but should be monitored in a three-monthly clinical and microbiological follow-up.

After the publication of the Dutch guideline, the approach was challenged by the head of the French reference laboratory for Rickettsioses, professor Raoult, who proposed another diagnostic guideline, in which the term chronic $Q$ fever was divided in four categories: the first two being definite and po ssible $Q$ fever endocarditis, while the second two were definite and possible $Q$ 
fever vascular infection [24]. Raoult refuted our claim that the tripartite system would yield high sensitivity and disagreed with our antibiotic indications; in his opinion, antibiotic treatment should not be allocated for proven chronic $Q$ fever patients only. In chapter 5, we compared the two guidelines and showed that with Raoult's guideline, approximately $30 \%$ of Dutch proven cases would have been missed and almost all probable and possible cases. These differences are mainly ascribed to the use of a higher IgG phase I cutoff and differences in the weighing of $C$. burnetii DNA detection in peripheral blood, which - in our assessment - produces definite proof.

With the Dutch guideline, practitioners were supported in their decision to start antibiotic treatment or not [16]. Serology, with IFAT IgG phase I as a cornerstone, remained an important diagnostic tool. And it was demonstrated that high IgG phase I titer and proven chronic $Q$ fever were strongly associated [25]. However, the specificity of the threshold is low. In different reports the clinical significance of high IgG phase I titers was criticized, especially in asymptomatic patients with risk factors for chronic $Q$ fever [26]. Also, in daily practice, it was observed that patients on treatment with sustained clinical response did not coincide with a decline in IFAT IgG phase I titer.

Given these limitations, there was a need to consider alternative techniques in order to find improved serological correlated patient management. Therefore, we conducted a study, described in chapter 6, comparing IFAT, ELISA and CFT for the detection of $C$. burnetii IgG phase I and IgA phase I in earlyand follow-up serum samples from patients with chronic $Q$ fever. For this, we collected sera of 49 patients of which 30 patients were classified as proven, 14 as probable and five as possible cases. At time of diagnosis, IgG phase I antibodies were missed using CFT in eight out of 49 patients. Five of them were categorized as proven. In this patient category, ELISA results were in full agreement with the IFAT. Instead, with ELISA three probable patients were missed. A (non-significant) difference in ELISA IgA phase I detection between proven chronic $Q$ fever and probable/possible was observed. Serological examination using ELISA en CFT in follow-up sera from 26 patients on treatment was unsatisfactory. Like IFAT, all kinetic options were possible: decreasing, remaining stable or even increasing during time. No differences were found when patients were categorized based on their clinical course. This study demonstrated that IgG phase I antibody detection with CFT is of no importance in the diagnosis of chronic $Q$ fever. ELISA IgG phase I has a 
limited value, but could have a place in a screening setting. Based on our results, there is no place for CFT or ELISA in the follow-up examination of chronic $Q$ fever patients.

\section{Vascullar wall infection}

The major organs involved in ongoing $Q$ fever are the heart valves and (aortic) vascular walls. Especially vascular wall infections can lead to severe complications and high mortality [22, 27]. From 2010, we were challenged by this new clinical picture, and to increase our knowledge we conducted a review of published cases with $Q$ fever vascular wall infections. In chapter 7 the results of this review combined with four cases at our institute are described [28]. We identified 58 cases with (aortic or peripheral) aneurysms and (aortic or peripheral) vascular grafts. Important symptoms mentioned were pain $(74 \%)$ and fever (69\%). Patients frequently presented with vascular complications, like vascular ruptures fistulas, para-aortic abscesses and thromboembolic complications. Forty patients were treated surgically and ten patients were treated conservatively. A total of 14 (24\%) fatalities were observed. The mortality in the conservatively treated group was significantly higher than in the group of patients whose infected aneurysms or grafts had been resected. Therefore, we conclude that surgical excision of the affected vascular segment must be an integral part of the treatment of vascular wall infections in cases of chronic $Q$ fever.

Another manifestation of chronic $Q$ fever is described in chapter 8 . In this particular example, the importance of surgical intervention is illustrated by the case of a woman with a three-year old Bentall prosthesis, inserted because of a thoracic aortic aneurysm that became infected with $C$. burnetii [29]. Prolonged antibiotic treatment failed and a new aortic tube was placed. During the operation, it became apparent that significant amounts of pus had gathered around the prosthesis. Hence, we were led to conclude that the treatment failure was caused either by an acquired resistance during longlasting antibiotic treatment or as a result of the fact that the infection was situated at a polyester prosthesis in an anatomical position with low antibiotic penetration.

We were confronted with more chronic $Q$ fever patients in a short period of time. The majority of cases had vascular wall infections and five patients died shortly after admission due to severe complications. Notably, most 
chronic $Q$ fever patients had never been diagnosed with acute $Q$ fever. The high number of serious chronic infections led us to investigate whether screening would yield an earlier detection, with the possible benefit of a better prognosis. In chapter $\mathbf{9}$ we showed that a screening program for chronic $Q$ fever after an acute $Q$ fever outbreak appears to be a good approach in detecting asymptomatic chronic $Q$ fever patients at an early stage [30]. Screening was limited to patients with abdominal and/or thoracic aortic aneurysms, vascular prostheses, or heart valve prostheses. These conditions were highly present in the symptomatic chronic $Q$ fever cases that were found in our region. In a cohort of 763 patients, ten new chronic $Q$ fever patients were found, of which two were cases of proven and eight of probable chronic $Q$ fever. Prostheses appeared to be a significant risk factor. All patients had (endo-)vascular prostheses of the aorta or heart valve prostheses and no conservatively treated aneurysms. Only one patient recorded symptoms, seven patients were started with specific anti $Q$ fever antibiotic treatment. Although a cost-effectiveness analysis is lacking in our study, it is conceivable that a screening program, such as initiated at our institution, will prove cost-effective. One can imagine that the cost of serology testing per patient is negligible, compared to the costs of treatment of symptomatic chronic $Q$ fever patients. The screening costs per person were low, approximately $€ 10$, in contrast with the costs of treatment, admission and operation procedure of a symptomatic chronic $Q$ fever patient.

\section{General discussion}

By conjoining knowledge and proposing research questions together with researchers in the field, we were able to provide answers to some of the pressing questions. However, these also yielded new questions that need to be answered. The diagnostics of acute $Q$ fever turned out to be straightforward in the epidemic situation. When executed adequately and when serology is combined with PCR, all serological techniques used in the Netherlands lead to practical and useful algorithms. In contrast with acute $Q$ fever, the utility of the same diagnostic tests in chronic $Q$ fever is limited due to low prospective values and cut-off variability (serology) as well as low sensitivity (PCR).

The major disadvantage of serology, which will remain a cornerstone in $Q$ fever diagnostics, is the long-lasting half-life time of antibodies. IgG as well 
as IgM antibodies are traceable in patient's sera years after the initial infection. This phenomenon, described in chapter 2 of this thesis as well as by other researchers, is mainly observed using IFAT [18, 31-33]. There is a significant difference in detection of antibodies in sera between IFAT and the other two techniques, ELISA and CFT [18]. Raising or lowering the cut-off could be an instrument to adapt IFAT to the current epidemiological situation. However, this is rather arbitrary and confronts laboratories with practical dilemmas; because high and low endemic regions can be present in the same area of coverage. In our opinion, it is better to choose a diagnostic test that is fitting with the diagnostic indication. For instance, for seroprevalence studies or for the determination of the burden of disease, it is necessary to use a test with high overall sensitivity [34]. For the purpose of vaccination strategies, high sensitivity is also required: Although the current vaccine exists of whole-cell, inactivated bacteria, vaccinating subjects who already have immunity can result in severe local reactions, such as abscesses [35]. Therefore, skin and blood tests should be done before vaccination to identify pre-existing immunity. In these cases, IFAT is the first line test.

In routine practice, the diagnostic indication differs from the aforementioned examples. In case of acute $Q$ fever, long-lasting sensitivity is not a required characteristic of the test, and could even raise the risk of false positivity. In these cases, CFT and ELISA are good alternatives. In our laboratory, we have chosen CFT and ELISA over IFAT for the purpose of acute $Q$ fever serological diagnostics and IFAT for chronic $Q$ fever. This is a practical procedure; however requests should be carefully examined by experienced technicians, because it occurs that physicians mix up acute and chronic $Q$ fever, making the choice of test not so straightforward.

In chapter 3 of this thesis, we proposed a dynamic notification rule reflecting the real time situation. It is of course difficult to point out an exact point in time to change the notifications rule. Perhaps a distinction between an epidemic and a non-epidemic rule is sufficient: in the former situation, active source finding is the main reason for notification. In this setting, reporting early and suspected cases will help to achieve this goal. In the latter case a non-epidemic setting or a post-epidemic setting, as is the case in parts of the Netherlands - the main purpose of reporting is monitoring. In our opinion, it suffices to downsize the notification rule to reporting only true cases in such situation. New $Q$ fever outbreaks are possible in the Netherlands, as the main source of the 2007-2009 outbreak, i.e. dairy goat farms, are still 
situated to a large extent near populated areas. Therefore, goat farms are examined for $C$. burnetii traces in milk tanks on a frequent basis [6, 36]. When tank milk samples prove positive for $C$. burnetii or when a sudden rise of true patients is detected during monitoring, the notification rule must be changed back to the epidemic standard.

The major diagnostic problems are mainly seen in chronic $Q$ fever. The limitations of serology and DNA detection were demonstrated in this thesis. We do think that the tripartite classification helps clinicians in their decisionmaking of whether or not to treat patients with antibiotics [16]. However, when in doubt, our current diagnostic tests are not discriminative enough to support therapeutic solutions. For example, in patients with risk factors, like vascular or heart valve prostheses, with only serological proof of chronic $Q$ fever, physicians are faced with dilemmas. It is worthy of note that these patients are mostly picked out in screening programs and frequently have no complaints nor do abnormalities come to the fore with diagnostic imaging techniques. We assume that these patients are better off with antibiotics, because of high IgG phase I titers, but the evidence for this is lacking. In two recent studies it has been shown that the positive predictive value of chronic $Q$ fever increases with the IgG phase I titer [25, 37]. In the study of Kampschreur et al., it was shown that there was a strong association between high phase I IgG titers and proven chronic $Q$ fever. In comparing patients with proven chronic $Q$ fever to cases of probable and possible chronic $Q$ fever, an increased positive predictive value (PPV) was found with increasing IgG phase I titer, with a PPV maximum of $86.2 \%$ in patients with a IgG phase I titer $\geq 1: 8192$. This study showed that the level of IFAT IgG phase I can indeed be used to support the decision to treat chronic $Q$ fever patients [25]. Unfortunately, the reverse does not hold true: a negative prospective value correlates only poorly to IFAT IgG titers. Therefore, it is recommended to keep the Dutch cut-off of IFAT IgG phase I $\geq 1: 1024$ in order not to miss any chronic $Q$ fever patients, because of the disease's high morbidity and mortality.

From the results of our comparative study between different assays described in chapter 6, the use of CFT in the diagnosis of chronic $Q$ fever is not advisable, because of low sensitivity. Due to the qualitative method, instead of quantitative, ELISA is dissuaded as well. It should be interesting making a quantitative IgG phase I ELISA test: in contrast with IFAT and CFT, intra- and inter-laboratory variations would be of no concern [32, 33, 38]. 
Moreover, half-life time of antibodies measured with ELISA are shorter than measured with IFAT, which suggests that ELISA-based testing reflects the disease's activity better [18, 34]. However, it is reasonable to assume that lack of discriminative power between proven and probable chronic $Q$ fever is independent the serological test used. Other, more sophisticated tools are urgently needed. For example the use of interferon-y production in the diagnosis of chronic $Q$ fever. T-lymphocyte mediated immunity plays an important role in eliminating the bacterium. In chronic infection, a disturbed immune system enables $C$. burnetii to multiply in macrophages. Interleukins, like interferon-gamma probably play an important role in this phenomenon [39-41]. Therefore, it should be interesting to investigate the kinetics of these interleukins during the follow-up of chronic $Q$ fever patients. Recently, it was shown that the correlation between IFAT IgG phase I and interferon-y was moderate [42]. Improvement of the interferon-y assay was established when combined with other interleukins, like IL-2 (unpublished data). This is only an example. The pool of interesting interleukins of cell mediated immunity is much bigger than interferon-y. However it is a good start and logically chosen because of its resemblance with the IGRA tests for Mycobacterium tuberculosis $[43,44]$.

Current serological tests are based on only phase I or phase II antigen composition. However, the scope of potential immunogenic antigens is probably much broader. With protein micro array techniques, it is possible to enlarge the amount of serological markers and thereby improving the discrimination between past and chronic infection [45-47]. This technique has been validated on Dutch sera containing samples from patients with acute, past and chronic infection. The used array contained $93 \%$ of the proteome of $C$. burnetii, with more than 2,000 different antigens [48]. Several serological markers were significantly present at higher levels in patients diagnosed with chronic $Q$ fever as compared to the acute patients. These detected markers may therefore be a better indicator for the chronic variant of the disease than the level of the IgG phase I in the Immuno Fluorescence Assay. Future research should be aimed at the discriminative characteristics of this technique between the different chronic $Q$ fever groups (proven, probable and possible).

The chronic $Q$ fever patients group is very diverse. There are differences in the organs involved, in co-morbidity and in the time point of diagnosis: some patients are diagnosed with symptomatic disease, others are traced in 
screening programs. This heterogeneity will challenge the further validation of the new diagnostic methodologies described above.

In daily practise, the treatment of patients with chronic $Q$ fever is hampered by a lack of guidelines. In chapter 7 of this thesis, we demonstrated that patients with vascular infections are better off when grafts are resected [28]. However, the optimal moment for surgical intervention is not determined yet and the decision to intervene is mostly based on a combination of factors, such as the physical state of the patient, a localised as well as systemic manifestation of the infection, co-morbidity and the availability of remaining alternative (conservative) options (e.g. a change in the antibiotic treatment regimen). Due to the deficiency of clinical as well as microbiological data, the criteria for surgical (re-) intervention are not straightforward and should be tailored to the individual patient as we described in chapter 8 [29]. Furthermore, it is unknown which type of surgical replacement for the infected vascular wall or graft is preferable. An autologous venous reconstruction appears to be the most appealing $[49,50]$. As an alternative for a deep venous reconstruction (a so-called Nevelsteen procedure) an autologous great saphenous spiral vein graft, appears promising in cases of infected aneurysms of the abdominal aorta. However, with this technique too, cases with complications and eventually death have been described. A recommendation for one of the available surgical techniques (extra-anatomical bypass surgery with (staged) explantation of the infected aneurysm with aggressive surgical debridement of the infected area, closure of the aortic stump and transmesenteric omentumplasty; in situ repair after aggressive surgical debridement with a cryopreserved arterial homograft, antibiotic coated (Silver) polyester grafts and subsequent transmesenteric omentumplasty; or one of the aforementioned autologous venous in situ repairs) cannot be given [51-53]. The heterogeneity and relative size of the patient group at risk precludes an answer to a preferred surgical technique. For the same reason guidelines are not expected at short notice. Maybe retrospective analysis based on the data collected in the Dutch chronic $Q$ fever database could give clues for best practise. In the mean time it is advisable to discuss cases with therapeutic problems in a multicentre multidisciplinary way.

In chapter 9, we showed that the prevalence of chronic $Q$ fever among serological positive patients was $19 \%$. This is in accordance with other Dutch screening programs [54]. The patients who were detected with a past infection ( $n=42$ ) with our screening program have been followed at regular inter- 
vals for two to three years now. A serological exacerbation or a positive $C$. burnetii PCR was found in none of the sera (unpublished data). It has been suggested in the literature that $C$. burnetii can reach a dormant stage and can reactivate years after the infection [55], implying that patients with a past serological profile may very well develop a chronic serological profile, months or years after the acute infection. However, we hypothesize that chronic infection is a slow, continuous process that follows the acute symptomatic infection. In other words, patients who develop a chronic serological profile do so very soon after an acute infection. It remains an open question why an infection develops into a chronic disease in one patient and simply stops in another patient with comparable risk factors. Firstly, gender is probably a risk factor, since most (chronic) $Q$ fever patients are male, and it is a well-established fact in the literature that males run a higher risk of developing symptomatic diseases. Female hormones may confer a protective role $[56,57]$. Secondly, in some of the literature, particular significance is given to the genotype of the bacterium. Eight genomic groups and 36 genotypes have been identified [58]. Association between genotypes with only acute $Q$ fever, only chronic $Q$ fever or both has been reported [59, 60]. However such an association is difficult to assess for a disease that is either sporadic or related to outbreaks. A multitude of factors is involved in determining whether someone will obtain an infection. This ranges from climatic factors, such as temperature, wind, humidity; where and how animals are kept (near densely populated areas, the number of animals per square meter); where and how they are slaughtered; how the manure is processed and used, and host factors. For example the variability of genotypes in a country is highly dependent on the goal for which the animals are bred and held. In the Netherlands, goat dairy farms are habitually highly specialised in only one part of the process of keeping goats. Some farms only specialise in breeding, whereas others only in raising goats. Most farms however focus solely on keeping goats for dairy production. The latter works with only one particular breed of goats that yield the highest milk production and that are obtained from a few specialised breeding farms. As a result, the Dutch goat population was almost "monoclonal", and hence, one strain of Coxiella was able to spread throughout the country. Therefore, in the Dutch outbreak, only one particular genotype was found in patients with acute $Q$ fever, as well as in the chronic patients [61]. In contrast, in Spain for example, there is a multitude of goat breeds - some kept for dairy, others for meat production, still 
others for both. These animals are often bred, raised and kept in one farm, with much less interaction between the farms leading to a higher variability of Coxiella genotypes [57]. Thirdly, the inoculation dose in the initial infections might be of importance as well, as was hypothesized for acute $Q$ fever before [62]. But if this is true, acute $Q$ fever syndrome should be preceding chronic infection, which is not the case. On the contrary: most chronic $Q$ fever patients cannot recall symptoms matching acute $Q$ fever in the years preceding (the diagnosis with) the chronic illness [54, 63]. The influence of the host's genetic make-up is poorly understood for chronic $Q$ fever, but may very well hold vital keys to resolving the puzzle. As in the case of Mycobacterium infections, the efficiency of cellular immunity is crucial. It is known from the pathogenesis of Mycobacteria that IL-12/IL-23/IFN-gamma axis plays an essential role in the cell-mediated immune response required for the elimination of pathogens $[43,44]$. Mutations in genes involved in the IL12/IL-23/IFN-gamma axis are known to cause extreme susceptibility to infection with certain types of common intracellular bacteria, including environmental Mycobacteria and Salmonella spp. It can be hypothesized that more frequent, less severe, genetic variations (single nucleotide polymorphisms, SNPs) in these immune response genes influence the susceptibility to persistence of $C$. burnetii infection. In line with SNP analysis in chronic $Q$ fever patients and appropriate control individuals, it should be possible to test this hypothesis. Currently, Dutch researchers from UMCN and UMCL are investigated this. The results of their research could be helpful for therapeutic triaging.

Although antibiotic treatment for chronic $Q$ fever was not a part of this thesis, the duration of treatment is based on practical experiences from France, where patients are treated at least 18 or 24 months based on the organs involved [64, 65]. In addition, IgG phase I titer must be declined fourfold before stopping antibiotics $[64,65]$. Due to the rareness of the disease, it is understandable that randomised controlled trials are lacking. In daily practice, it is noticed that the decline of IgG phase I is hardly observed in patients during treatment. If IgG phase I titer really reflects the status of infection, this could mean that antibiotics lead to a sustained situation rather than to recovery. The preferred treatment of doxycycline with hydroxyplaquenil is not based on human studies, but mainly tested in vitro [66, 67]. In fact, for prolonged treatment only doxycycline is given, because hydroxyplaquenil has no bactericidal or bacteriostatic activity. Although not tested in the Nether- 
lands, one can imagine that this monotherapy can lead to resistance. Again, we need more and better prognostic parameters to follow chronic $Q$ fever patients and for the support of finishing treatment. Until new techniques are implemented in routine testing, serology remains the most important tool, as it is frankly the only one we have.

In chapter 9 we showed that with our screening program, asymptomatic chronic $Q$ fever patients could be detected at an early stage. We assumed that the outcome of these patients would be better than symptomatic patients. The former group presented frequently with severe complications of chronic $Q$ fever, such as infected grafts, abscesses and fistulas wherefore complex, sometimes multiple surgical procedures were necessary. Main purpose of the screening program was to decrease the risk for these complications by early detection and antibiotic treatment. It was suggested that screening for chronic $Q$ fever should be introduced nationally. However, before implementing screening at a national level, effectiveness should be evaluated, including a cost analysis. In the Netherlands, chronic $Q$ fever patients are included and followed in a national database. With this database, comparison can be made between the outcome (mortality and morbidity) of patients detected by screening with the outcome of those presenting with symptomatic chronic $Q$ fever.

\section{Lessons learned from the Dutch Q fever outbreak}

New outbreaks are possible in the Netherlands, because of intensive livestock industry neighbouring human communities. The severe and prolonged sequential outbreaks of $Q$ fever in the Netherlands urged policy makers to have a close control on $C$. burnetii traces in cowsheds. From the human point of view, one can question whether it is sufficient to only monitor livestock. It should be considered whether an additional method of human examination could be beneficial to the control of $C$. burnetii. One could think, for instance, of periodical screening of people living in an area close to goat farms. This could either be a general screening or a screening of people with risks for chronic $Q$ fever. $Q$ fever vaccination is another option worth considering. Considerations such as these touch on the fundamental question how much further the (Dutch) livestock industry can expand near densely populated areas, without putting the community health at risk. Close interaction 
between the veterinary and human health services and communities is hence required.

\section{Conclusion}

In this thesis, the value of different current diagnostic tests for acute and chronic $Q$ fever has been determined. The use of serology and PCR are sufficient for diagnosing acute $Q$ fever, especially when both tests are combined in an algorithm. Due to long-lasting half time of antibodies, adaptations of algorithms as well as of interpretations of test results are required in postepidemic areas. The current tests are inadequate for diagnosing chronic $Q$ fever: the sensitivity of the PCR test used in the Netherlands is very low and serological results fail to discriminate between different chronic $Q$ fever groups sufficiently. The diagnosis of chronic $Q$ fever is therefore challenging and should not merely be based on these microbiological tests, but also on clinical aspects and diagnostic imaging results. Triaging the probability of chronic $Q$ fever helps clinicians in their therapeutic decision-making. But, new tests, based on different principles, are urgently needed, also for the follow-up of chronic $Q$ fever patients.

Besides diagnostic analyses, clinical aspects of chronic $Q$ fever patients were generated in this thesis. We showed that surgical intervention should always be considered as a part of the treatment of vascular infected patients. The existence of asymptomatic chronic $Q$ fever was demonstrated during a $Q$ fever screening program. Further expediency study is necessary to examine whether the screenings program was (cost) effective.

Some of the studies in this thesis were embedded in the national $Q$ fever consensus group. This multidisciplinary approach is exportable to future outbreaks with emerging pathogen: firstly, install a team of experts in the field. Secondly, collect and share the available knowledge and thirdly, determine together the main 'blank spots' for which research should be initiated. 


\section{References}

1. Delsing CE, Kullberg BJ. Q fever in the Netherlands: a concise overview and implications of the largest ongoing outbreak. Neth J Med 2008 Oct;66(9):365-7.

2. Delsing CE, Kullberg BJ, Bleeker-Rovers CP. Q fever in the Netherlands from 2007 to 2010. Neth J Med 2010 Dec;68(12):382-7.

3. Karagiannis I, Morroy G, Rietveld A, Horrevorts AM, Hamans M, Francken P, et al. Q fever outbreak in the Netherlands: a preliminary report. Euro Surveill 2007 Aug;12(8):E070809.

4. Schimmer B, Morroy G, Dijkstra F, Schneeberger PM, Weers-Pothoff G, Timen A, et al. Large ongoing $Q$ fever outbreak in the south of The Netherlands, 2008. Euro Surveill 2008 Jul 31;13(31).

5. Schimmer B, Dijkstra F, Vellema P, Schneeberger PM, Hackert V, ter SR, et al. Sustained intensive transmission of $Q$ fever in the south of the Netherlands, 2009. Euro Surveill 2009 May 14;14(19).

6. van den BR, van EE, Luttikholt S, Moll L, van MK, Vellema P. Coxiella burnetii in bulk tank milk samples from dairy goat and dairy sheep farms in The Netherlands in 2008. Vet Rec 2012 Mar 24;170(12):310.

7. van den BR, Moll L, van SG, Vellema P. Demography of $Q$ fever seroprevalence in sheep and goats in The Netherlands in 2008. Prev Vet Med 2013 Apr 1;109(1-2):76-82.

8. van der Hoek W, Dijkstra F, Schimmer B, Schneeberger PM, Vellema P, Wijkmans C, et al. $Q$ fever in the Netherlands: an update on the epidemiology and control measures. Euro Surveill 2010 Mar 25;15(12).

9. Parker NR, Barralet JH, Bell AM. Q fever. Lancet 2006 Feb 25;367(9511):679-88.

10. Schimmer B, ter SR, Wegdam M, Zuchner L, De BA, Schneeberger PM, et al. The use of a geographic information system to identify a dairy goat farm as the most likely source of an urban Q-fever outbreak. BMC Infect Dis 2010;10:69.

11. Dupuis G, Petite J, Peter O, Vouilloz M. An important outbreak of human Q fever in a Swiss Alpine valley. Int J Epidemiol 1987 Jun;16(2):282-7.

12. Hawker JI, Ayres JG, Blair I, Evans MR, Smith DL, Smith EG, et al. A large outbreak of Q fever in the West Midlands: windborne spread into a metropolitan area? Commun Dis Public Health 1998 Sep;1(3):180-7.

13. Porten K, Rissland J, Tigges A, Broll S, Hopp W, Lunemann M, et al. A super-spreading ewe infects hundreds with $Q$ fever at a farmers' market in Germany. BMC Infect Dis 2006;6:147.

14. Hogema BM, Slot E, Molier M, Schneeberger PM, Hermans MH, van Hannen EJ, et al. Coxiella burnetii infection among blood donors during the 2009 Q-fever outbreak in The Netherlands. Transfusion 2012 Jan;52(1):144-50.

15. Wegdam-Blans MC, Nabuurs-Franssen MN, Horrevorts AM, Peeters MF, Schneeberger PM, Bijlmer HA. [Laboratory diagnosis of acute Q fever]. Ned Tijdschr Geneeskd 2010;154:A2388.

16. Wegdam-Blans MC, Kampschreur LM, Delsing CE, Bleeker-Rovers CP, Sprong T, van Kasteren ME, et al. Chronic $Q$ fever: review of the literature and a proposal of new diagnostic criteria. J Infect 2012 Mar;64(3):247-59.

17. Herremans T, Hogema BM, Nabuurs M, Peeters M, Wegdam-Blans M, Schneeberger P, et al. Comparison of the performance of IFA, CFA, and ELISA assays for the serodiagno- 
sis of acute Q fever by quality assessment. Diagn Microbiol Infect Dis 2013 Jan;75(1):1621.

18. Wegdam-Blans MC, Wielders CC, Meekelenkamp J, Korbeeck JM, Herremans T, Tjhie $\mathrm{HT}$, et al. Evaluation of commonly used serological tests for detection of Coxiella burnetii antibodies in well-defined acute and follow-up sera. Clin Vaccine Immunol 2012 Jul;19(7):1110-5.

19. Q-koorts, Dutch 2010. (http://www.rivm.nl/dsresource?objectid=rivmp:6826\&type=org\&disposition=inline)

20. Brouqui $P$, Dupont HT, Drancourt M, Berland Y, Etienne J, Leport C, et al. Chronic Q fever. Ninety-two cases from France, including 27 cases without endocarditis. Arch Intern Med 1993 Mar 8;153(5):642-8.

21. Million M, Thuny F, Richet H, Raoult D. Long-term outcome of $Q$ fever endocarditis: a 26year personal survey. Lancet Infect Dis 2010 Aug;10(8):527-35.

22. Raoult D, Tissot-Dupont H, Foucault C, Gouvernet J, Fournier PE, Bernit E, et al. Q fever 1985-1998. Clinical and epidemiologic features of 1,383 infections. Medicine (Baltimore) 2000 Mar;79(2):109-23.

23. Fenollar F, Fournier PE, Carrieri MP, Habib G, Messana T, Raoult D. Risks factors and prevention of $Q$ fever endocarditis. Clin Infect Dis 2001 Aug 1;33(3):312-6.

24. Raoult $D$. Chronic $Q$ fever: expert opinion versus literature analysis and consensus. $J$ Infect 2012 Aug;65(2):102-8.

25. Kampschreur LM, Oosterheert JJ, Koop AM, Wegdam-Blans MC, Delsing CE, BleekerRovers $\mathrm{CP}$, et al. Microbiological challenges in the diagnosis of chronic $Q$ fever. Clin Vaccine Immunol 2012 May;19(5):787-90.

26. van der Hoek W, Versteeg B, Meekelenkamp JC, Renders NH, Leenders AC, WeersPothoff I, et al. Follow-up of 686 patients with acute $Q$ fever and detection of chronic infection. Clin Infect Dis 2011 Jun 15;52(12):1431-6.

27. Botelho-Nevers E, Fournier PE, Richet H, Fenollar F, Lepidi H, Foucault C, et al. Coxiella burnetii infection of aortic aneurysms or vascular grafts: report of 30 new cases and evaluation of outcome. Eur J Clin Microbiol Infect Dis 2007 Sep;26(9):635-40.

28. Wegdam-Blans MC, Vainas T, van Sambeek MR, Cuypers PW, Tjhie HT, van Straten $\mathrm{AH}$, et al. Vascular complications of Q-fever infections. Eur J Vasc Endovasc Surg 2011 Sep;42(3):384-92.

29. Wegdam-Blans MC, ter Woorst JF, Klompenhouwer EG, Teijink JA. David procedure during a reoperation for ongoing chronic $Q$ fever infection of an ascending aortic prosthesis. Eur J Cardiothorac Surg 2012 Jul;42(1):e19-e20.

30. Wegdam-Blans MC, Stokmans RA, Tjhie JH, Korbeeck JM, Koopmans MP, Evers SM, et al. Targeted screening as a tool for the early detection of chronic $Q$ fever patients after a large outbreak. Eur J Clin Microbiol Infect Dis 2012 Sep 26.

31. Embil J, Williams JC, Marrie TJ. The immune response in a cat-related outbreak of $Q$ fever as measured by the indirect immunofluorescence test and the enzyme-linked immunosorbent assay. Can J Microbiol 1990 Apr;36(4):292-6.

32. Field PR, Hunt JG, Murphy AM. Detection and persistence of specific IgM antibody to Coxiella burnetii by enzyme-linked immunosorbent assay: a comparison with immunofluorescence and complement fixation tests. J Infect Dis 1983 Sep;148(3):477-87.

33. Field PR, Santiago A, Chan SW, Patel DB, Dickeson D, Mitchell JL, et al. Evaluation of a novel commercial enzyme-linked immunosorbent assay detecting Coxiella burnetii- 
specific immunoglobulin $\mathrm{G}$ for $\mathrm{Q}$ fever prevaccination screening and diagnosis. J Clin Microbiol 2002 Sep;40(9):3526-9.

34. Blaauw GJ, Notermans DW, Schimmer B, Meekelenkamp J, Reimerink JH, Teunis P, et al. The application of an enzyme-linked immunosorbent assay or an immunofluorescent assay test leads to different estimates of seroprevalence of Coxiella burnetii in the population. Epidemiol Infect 2012 Jan;140(1):36-41.

35. Gidding HF, Wallace C, Lawrence GL, Mclntyre PB. Australia's national Q fever vaccination program. Vaccine 2009 Mar 23;27(14):2037-41.

36. Muskens J, van EE, van MC, Bartels C, Lam TJ. Prevalence of Coxiella burnetii infection in Dutch dairy herds based on testing bulk tank milk and individual samples by PCR and ELISA. Vet Rec 2011 Jan 22;168(3):79.

37. Frankel D, Richet H, Renvoise A, Raoult D. Q fever in France, 1985-2009. Emerg Infect Dis 2011 Mar; 17(3):350-6.

38. D'Harcourt SC, Soto AB, Burgos VC, Calero DL, Martinez-Zapico R. Comparison of immunofluorescence with enzyme immunoassay for detection of $Q$ fever. Eur $\mathrm{J}$ Clin Microbiol Infect Dis 1996 Sep;15(9):749-52.

39. Capo C, Mege JL. Role of innate and adaptive immunity in the control of $Q$ fever. Adv Exp Med Biol 2012;984:273-86.

40. Dellacasagrande J, Capo C, Raoult D, Mege JL. IFN-gamma-mediated control of Coxiella burnetii survival in monocytes: the role of cell apoptosis and TNF. J Immunol 1999 Feb 15;162(4):2259-65.

41. Dellacasagrande J, Ghigo E, Raoult D, Capo C, Mege JL. IFN-gamma-induced apoptosis and microbicidal activity in monocytes harboring the intracellular bacterium Coxiella burnetii require membrane TNF and homotypic cell adherence. J Immunol 2002 Dec 1;169(11):6309-15.

42. Schoffelen T, Joosten LA, Herremans T, de Haan AF, Ammerdorffer A, Rumke HC, et al. Specific interferon gamma detection for the diagnosis of previous $Q$ fever. Clin Infect Dis 2013 Jun;56(12):1742-51.

43. van d, V, Hoeve MA, Ottenhoff TH. Human genetics of intracellular infectious diseases: molecular and cellular immunity against mycobacteria and salmonellae. Lancet Infect Dis 2004 Dec;4(12):739-49.

44. van d, V, van Dissel JT, Ottenhoff TH. Genetic deficiencies of innate immune signalling in human infectious disease. Lancet Infect Dis 2009 Nov;9(11):688-98.

45. Beare PA, Chen C, Bouman T, Pablo J, Unal B, Cockrell DC, et al. Candidate antigens for $Q$ fever serodiagnosis revealed by immunoscreening of a Coxiella burnetii protein microarray. Clin Vaccine Immunol 2008 Dec;15(12):1771-9.

46. Vigil A, Ortega R, Nakajima-Sasaki R, Pablo J, Molina DM, Chao CC, et al. Genome-wide profiling of humoral immune response to Coxiella burnetii infection by protein microarray. Proteomics 2010 Jun;10(12):2259-69.

47. Vigil A, Chen C, Jain A, Nakajima-Sasaki R, Jasinskas A, Pablo J, et al. Profiling the humoral immune response of acute and chronic $Q$ fever by protein microarray. Mol Cell Proteomics 2011 Oct;10(10):M110.

48. Herremans T, Vigil A, Jain A, Nijhuis C, Schoffelen T, Nabuurs-Franssen MH, et al. profiling the antibody respons in acute and chronic patients from a recent Dutch $Q$ fever outbreak by protein microarray. 2012. Ref Type: Unpublished Work 
49. Ehsan O, Gibbons CP. A 10-year experience of using femoro-popliteal vein for revascularisation in graft and arterial infections. Eur J Vasc Endovasc Surg 2009 Aug;38(2):172-9.

50. Heikens JT, Coveliers HM, Burger DH, van Berge Henegouwen DP, Vriens PW. Saphenous vein spiral graft: successful emergency repair of a mycotic aneurysm with aortoduodenal fistula. Eur J Vasc Endovasc Surg 2006 Oct;32(4):408-10.

51. Kloppenburg GT, van de Pavoordt ED, de Vries JP. Endograft-preserving therapy of a patient with Coxiella burnetii-infected abdominal aortic aneurysm: a case report. J Med Case Rep 2011;5:565.

52. Murphy EH, Szeto WY, Herdrich BJ, Jackson BM, Wang GJ, Bavaria JE, et al. The management of endograft infections following endovascular thoracic and abdominal aneurysm repair. J Vasc Surg 2013 Jul 4.

53. O'Connor S, Andrew P, Batt M, Becquemin JP. A systematic review and meta-analysis of treatments for aortic graft infection. J Vasc Surg 2006 Jul;44(1):38-45.

54. Kampschreur LM, Hagenaars JC, Wielders CC, Elsman P, Lestrade PJ, Koning OH, et al. Screening for Coxiella burnetii seroprevalence in chronic $Q$ fever high-risk groups reveals the magnitude of the Dutch $Q$ fever outbreak. Epidemiol Infect 2012 Jun 13;1-5.

55. Harris RJ, Storm PA, Lloyd A, Arens M, Marmion BP. Long-term persistence of Coxiella burnetii in the host after primary $Q$ fever. Epidemiol Infect 2000 Jun;124(3):543-9.

56. Leone M, Honstettre A, Lepidi H, Capo C, Bayard F, Raoult D, et al. Effect of sex on Coxiella burnetii infection: protective role of 17beta-estradiol. J Infect Dis 2004 Jan 15;189(2):339-45.

57. Raoult D, Marrie T, Mege J. Natural history and pathophysiology of $Q$ fever. Lancet Infect Dis 2005 Apr;5(4):219-26.

58. Beare PA, Samuel JE, Howe D, Virtaneva K, Porcella SF, Heinzen RA. Genetic diversity of the $Q$ fever agent, Coxiella burnetii, assessed by microarray-based whole-genome comparisons. J Bacteriol 2006 Apr;188(7):2309-24.

59. Jado I, Carranza-Rodriguez C, Barandika JF, Toledo A, Garcia-Amil C, Serrano B, et al. Molecular method for the characterization of Coxiella burnetii from clinical and environmental samples: variability of genotypes in Spain. BMC Microbiol 2012;12:91.

60. Santos AS, Tilburg JJ, Botelho A, Barahona MJ, Nuncio MS, Nabuurs-Franssen MH, et al. Genotypic diversity of clinical Coxiella burnetii isolates from Portugal based on MST and MLVA typing. Int J Med Microbiol 2012 Nov;302(6):253-6.

61. Tilburg JJ, Roest HJ, Buffet S, Nabuurs-Franssen MH, Horrevorts AM, Raoult D, et al. Epidemic genotype of Coxiella burnetii among goats, sheep, and humans in the Netherlands. Emerg Infect Dis 2012 May;18(5):887-9.

62. Stein A, Louveau C, Lepidi H, Ricci F, Baylac P, Davoust B, et al. Q fever pneumonia: virulence of Coxiella burnetii pathovars in a murine model of aerosol infection. Infect Immun 2005 Apr;73(4):2469-77.

63. Kampschreur LM, Oosterheert JJ, Hoepelman Al, Lestrade PJ, Renders NH, Elsman P, et al. Prevalence of chronic $Q$ fever in patients with a history of cardiac valve surgery in an area where Coxiella burnetii is epidemic. Clin Vaccine Immunol 2012 Aug;19(8):1165-9.

64. Raoult D, Piquet P, Gallais H, de MC, Drancourt M, Casanova P. Coxiella burnetii infection of a vascular prosthesis. N Engl J Med 1986 Nov 20;315(21):1358-9. 
65. Raoult D, Houpikian P, Tissot DH, Riss JM, rditi-Djiane J, Brouqui P. Treatment of $Q$ fever endocarditis: comparison of 2 regimens containing doxycycline and ofloxacin or hydroxychloroquine. Arch Intern Med 1999 Jan 25;159(2):167-73.

66. Raoult D, Yeaman MR, Baca OG. Susceptibility of Coxiella burnetii to pefloxacin and ofloxacin in ovo and in persistently infected L929 cells. Antimicrob Agents Chemother 1989 May;33(5):621-3.

67. Raoult D, Drancourt M, Vestris G. Bactericidal effect of doxycycline associated with lysosomotropic agents on Coxiella burnetii in P388D1 cells. Antimicrob Agents Chemother 1990 Aug;34(8):1512-4. 


Samenvatting 
Tussen 2007 en 2009 vond in Nederland 's werelds grootste Q-koorts uitbraak plaats, met meer dan 4000 gemelde gevallen van acute Q-koorts. De zwaarst getroffen gebieden waren de provincies Noord-Brabant, Gelderland en Limburg. Melkgeit bedrijven werden geïdentificeerd als de belangrijkste bron van de Coxiella burnetii (C. burnetii) bacterie, de verwekker van $\mathrm{Q}$ koorts. Grote hoeveelheden van de bacterie kwamen vrij via geboorteproducten, zoals geaborteerde lammeren, placenta en vruchtwater. Er was een duidelijke jaarlijkse seizoenspiek; de meeste patiënten met acute Q-koorts werden gevonden tijdens het lammer seizoen in het voorjaar. Uiteindelijk werden maatregelen getroffen, zoals het ruimen van Q-koorts positieve geitenbedrijven en het vaccineren van nieuwe geiten. Hiermee werd de epidemie beëindigd eind 2009. Zowel de omvang als de duur van de Nederlandse uitbraak was van een ongekende schaal: drie jaar achtereen, werd een bevolking van drie provincies met ca 5,5 miljoen inwoners blootgesteld aan grote hoeveelheden $C$. burnetii.

Het aantonen van Q-koorts in het laboratorium gebeurt middels het aantonen van antistoffen in het bloed (serologische testen) en/of DNA van de $C$. burnetii bacterie in bloed of in weefsel met een PCR test. Met de toename van het aantal aanvragen en het aanhoudende karakter van de epidemie was er behoefte aan afstemming in de diagnostiek. Om die reden werd de Q-koorts consensus groep opgericht. Een initiatief van het Nederlandse Rijksinstituut voor Volksgezondheid en Milieu (RIVM) en de Nederland Vereniging voor Medische Microbiologie (NVMM). Deze groep werd geformeerd door artsen-microbioloog en klinisch specialisten, betrokken bij de diagnostiek en behandeling van Q-koorts. De consensus groep, in eerste instantie gericht voor acute Q-koorts, werd opgericht in 2010.

De in dit proefschrift beschreven studies zijn opgezet om verschillende laboratoriumtechnieken voor acute en chronische Q-koorts te vergelijken en om de toepasbaarheid van de verschillende testen in de dagelijkse praktijk te bepalen. Daarnaast beschrijft en categoriseert dit proefschrift klinische aspecten van chronische Q-koorts. Een aantal studies uit dit proefschrift kwamen voort uit de besprekingen van de Q-koorts consensusgroep (hoofdstuk 2, 3 en 4).

\section{Diagnose van acute $\mathrm{Q}=$ koorts}

Voor de diagnostiek van acute Q-koorts zijn de antistoffen IgM-fase II en IgG-fase II van belang, waarbij IgM-fase II eerder kan worden gedetecteerd 
in het bloed dan IgG-fase II. De hoeveelheid antistoffen worden uitgedrukt in een titerhoogte. Acute Q-koorts kan worden vermoed als Ig M-fase II antistoffen aanwezig zijn. Acute Q-koorts is bewezen als een significantie stijging is waar te nemen in de IgG-phase II titerhoogte of als DNA aantoonbaar is, middels een PCR-test in het bloed op tijdstip van de eerste klinische pres entatie (tijdstip 0). Voor de diagnostiek van Q-koorts zijn verschillende antistoftestmethoden beschikbaar in Nederland: indirecte Fluorescent Antibody Test (IFAT), enzyme-linked immunosorbent assay (ELISA) en complement fixatie test (CFT). De interpretatie van deze antistoftesten was niet altijd eenduidig, daardoor ontbrak, zeker in het begin van de uitbraak, uniformiteit in de diagnostiek. Daarom voerden we een studie uit, beschreven in hoofdstuk 2, waarin we de drie testen vergeleken in een groep van 126 patiënten. Zowel in het bloedmonster in de acute fase (tijdstip 0), als 3 maandelijkse vervolgmonsters van de patiënten werden getest. Op basis van IgG-fase II antistofbepaling in gepaarde bloedmonsters waren alle drie de testen vergelijkbaar in het vaststellen van acute Q-koorts. IgM-fase II was reeds aantoonbaar in een deel van de bloedmonsters in de acute fase. Echter, IgM-fase II was ook detecteerbaar in het merendeel van de bloedmonsters na 1 jaar (onze maximale observatieperiode). Significante verschillen werden aangetoond in de follow-up bloedmonsters. De afname in de titerhoogte van zowel Ig G-fase II als IgG-fase I antistoffen, was beduidend langzamer met IFAT in vergelijking met ELISA en CFT. De resultaten van deze studie geven aan dat de keuze van de test afhankelijk is van de indicatie. 1. In de gebieden van $\mathrm{Ne}$ derland die getroffen waren door de epidemie is de waarde van een positieve IgM-fase II in een bloedmonster zeer beperkt. Immers, deze IgMantistoffen kunnen reeds eerder zijn aangemaakt. Mensen die besmet raken met Q-koorts, hoeven hier niet altijd ziek van te worden maar vormen wel antistoffen, wat de interpretatie van de test bemoeilijkt. 2. Om acute Q-koorts vast te stellen is serologisch bewijs nodig, gebaseerd op basis van twee bloedmonsters. Onze resultaten toonden aan dat de verschillende testmethoden vergelijkbaar zijn in dit opzicht. 3. Doordat IFAT de hoogste gevoeligheid liet zien in late follow-up bloedmonsters, heeft deze test de voorkeur indien de vraagstelling van de test is of een patiënt ooit Q-koorts heeft doorgemaakt, bijvoorbeeld voorafgaand aan vaccinatie.

Q-koorts is een meldingspichtige ziekte. De belangrijkste reden voor de melding is bronopsporing. In Nederland is het meldingssysteem getrapt: na een eerste melding van een (vermoeden) op een besmettelijke ziekte door het 
laboratorium en/of behandelaar aan de GGD, beoordeelt de infectieziektenarts van de GGD of de melding ook voldoet aan klinische criteria. Als dat zo is wordt de melding herbevestigd en gerapporteerd in de nationale ziekte overzicht (OSIRIS). Voor Q-koorts geldt dat zowel laboratorium bewezen als laboratorium verdachte patiënten moeten worden gemeld aan de GGD. In hoofdstuk 3 hebben we een analyse beschreven, waarbij de percentages van de meldingen in OSIRIS tijdens en na de uitbraak zijn vergeleken. Op basis van de laboratoriumresultaten, kan acute Q-koorts gediagnosticeerd worden als: 1. bewezen (PCR-positief of significante stijging van IgG-fase II titerhoogte); 2. waarschijnlijk (enkelvoudige detectie van IgG- en IgM-fase II antistoffen); of 3. mogelijk (enkelvoudige detectie van IgM-fase II antistoffen). Onze hypothese was dat het aantal bevestigde gevallen in OSIRIS zou afnemen in de postepidemische gebieden als gevolg van detectie van antistoffen uit het verleden, zoals ook opgemerkt in hoofdstuk 2. In een retrospectieve studie (2009 t/m 2011) van 523 patiënten die door ons laboratorium aan de GGD waren gemeld, werd inderdaad een significante afname aangetoond van het percentage bevestigde acute Q-koorts patiënten in OSIRIS in 2009 ten opzichte van de twee jaren daarna; het percentage gemelde patiënten in OSIRIS daalde met 50\% in de jaren 2010 en 2011. In 2010 en 2011 bereikten bijna geen van de meldingen OSIRIS. Blijkbaar waren de meeste laboratoriummeldingen in de postepidemische jaren 2010 en 2011 gebaseerd op oude infecties, waarbij de antistoffen jaren later nog aantoonbaar bleken te zijn. Het melden van doorgemaakte infecties is niet zinvol en draagt niet bij tot de kernactiviteit van de GGD, namelijk bronopsporing. Op basis van onze resultaten is een verandering van de meldingscriteria aan te raden, waarbij, in ieder geval in de getroffen gebieden, alleen bewezen acute $\mathrm{Q}$-koorts zou moeten worden gemeld.

\section{Diagnose van chronische Q-koorts}

Nadat de acute Q-koorts epidemie in Nederland was beëindigd, werd een groeiend aantal gevallen van chronische Q-koorts infecties in de getroffen gebieden vastgesteld. Chronische Q-koorts verloopt meestal ongemerkt en kan zich maanden of zelfs jaren na de primaire besmetting presenteren. Meestal zijn de symptomen niet specifiek, zoals koorts, nachtzweten en gewichtsverlies. In tegenstelling tot acute Q-koorts, is chronische Q-koorts een potentieel dodelijke ziekte. Voor veel Nederlandse artsen was chronische Q-koorts een nieuw ziektebeeld. Met een snel groeiend aantal patiën- 
ten in een korte tijd, was er dringend behoefte aan uniformiteit in de diagnose van chronische Q-koorts. Om die reden werd een systematische review verricht van de beschikbare literatuur over de klinische aspecten en diagnostische testen van chronische Q-koorts. De belangrijkste uitkomst van deze review, beschreven in hoofdstuk 4, was dat de diagnose chronische Qkoorts moeilijk te stellen is. C. burnetii, de verwekker van Q-koorts, is niet kweekbaar. Daardoor zijn andere, vaak minder gevoelige technieken nodig. Zoals de PCR methode, waarbij DNA van de bacterie wordt aangetoond. Uit de analyse van de gepubliceerde artikelen over de gevoeligheid van de PCR voor chronische Q-koorts, kwam naar voren dat deze zeer matig was in het bloed. Met andere woorden, een negatieve PCR-test uit bloed sluit de diagnose, chronische Q-koorts absoluut niet uit. In tegenstelling tot bloed, is de gevoeligheid van de PCR test op weefsel, zoals hartklep of vaatwand $100 \%$. Maar in de dagelijkse praktijk zijn die materialen slechts zelden aanwezig. Chronische Q-koorts patiënten maken allemaal IgG-fase I antistoffen. Hoe hoger de titer, hoe meer waarschijnlijk de diagnose chronische Q-koorts is. Doordat de microbiologische diagnostiek niet sluitend is, zijn resultaten uit beeldvormend onderzoek (radiologie) en klinische symptomen ook belangrijk. De review was de basis voor een diagnostische richtlijn, waarbij microbiologische, maar ook radiologische en klinische gegevens worden meegewogen in het vaststellen van de diagnose. Op basis van deze gegevens worden patiënten ingedeeld in bewezen, waarschijnlijke en mogelijke chronische Q-koorts. Men spreekt van bewezen chronische Q-koorts indien DNA van C. burnetii aantoonbaar is in bloed en/of weefsel of in het geval de lgGfase I titer groter of gelijk is aan 1:1024 in combinatie met een positieve radiologische test van vaatwand en/of hartklep. Er is sprake van waarschijnlijk chronische Q-koorts bij patiënten met een IgG-fase I titer groter of gelijk aan 1:1024 in combinatie met risicofactoren voor chronische Q-koorts en negatieve beeldvorming. Patiënten worden geclassificeerd met mogelijk chronische Q-koorts indien IgG-fase I titer groter of gelijk is aan 1:1024 in afwezigheid van andere factoren. Langdurige behandeling met antibiotica moet altijd worden gestart in het geval van bewezen chronische Q-koorts. Bij patiënten met waarschijnlijke chronische Q-koorts moet de indicatie voor behandeling met antibiotica worden besproken in een multidisciplinair team. Patiënten met een mogelijke chronische Q-koorts hebben geen indicatie voor antibiotica, maar moeten wel driemaandelijks worden gecontroleerd op klinische manifestaties en microbiologische gegevens. 
Na de publicatie van de Nederlandse richtlijn, werd onze aanpak ter discussie gesteld door het hoofd van het Franse referentielaboratorium voor Rickettsiosis, professor Raoult. Hij publiceerde een andere indeling, die volgens de auteur gevoeliger zou zijn dan de Nederlandse richtlijn. In zijn publicatie wordt chronische Q-koorts opgedeeld in vier categorieën: definitieve en mogelijke Q-koorts endocarditis, en definitieve en mogelijke Q-koorts vaatwandinfectie. In hoofdstuk 5, vergeleken we de twee richtlijnen en toonden aan dat met de richtlijn Raoult ongeveer 30\% van de Nederlandse bewezen chronische Q-koorts patiënten zouden worden gemist. Daarnaast zouden ook bijna alle waarschijnlijke en mogelijke gevallen niet worden gediagnosticeerd met de richtlijn van Raoult. Dit resultaat werd voornamelijk toegeschreven aan de waarde van een positieve PCR-test, welke in de Nederlandse richtlijn, mits acute Q-koorts is uitgesloten, een bewezen infectie vaststelt.

Met de Nederlandse richtlijn in de hand werden internist-infectiologen gesteund in hun beslissing om een behandeling met antibiotica wel of niet te starten. Daarbij was de hoogte van de IgG-fase I antistoftiter, gemeten met IFAT, een belangrijk diagnostisch hulpmiddel: bewezen chronische Q-koorts en hoge titer IgG-fase I antistoffen zijn sterk geassocieerd. Echter, de specificiteit van IgG-fase I titer is laag. Of te wel, niet alle patiënten met hoge IgGfase I titer hebben een bewezen chronische Q-koorts. Daarnaast is de toepasbaarheid van de IgG-fase I titer als marker van het succes van antibiotische behandeling onduidelijk.

In hoofdstuk 6 onderzochten we of gebruik van andere serologische technieken additionele informatie kon genereren in het vaststellen van chronische Q-koorts en in de follow-up van patiënten. In deze studie vergeleken we bloedmonsters van 49 chronische Q-koorts patiënten: 30 patiënten bewezen, 14 waarschijnlijk en vijf mogelijke chronische Q-koorts gevallen. Voor het vaststellen van chronische Q-koorts bleken de alternatieve testen, CFT en ELISA, geen geschikte methoden: acht van de 49 patiënten, waar onder vijf bewezen chronische Q-koorts patiënten, werden gemist met CFT. ELISA deed het beter, maar met deze test werden drie, waarschijnlijke chronische Q-koorts patiënten, gemist. Ook het serologisch vervolgen van patiënten met antibiotica met andere technieken dan IFAT kon met dit onderzoek niet worden uitgevoerd. Kortom, op basis van dit onderzoek is geen plaats voor CFT of ELISA in het vaststellen of het vervolgen van chronische Q-koorts patiënten. 


\section{Vaatwand infectie}

De belangrijkste organen die zijn aangedaan bij chronische Q-koorts zijn de hartkleppen en de vaatwand van de aorta. Vooral infectie van de vaatwand kan leiden tot ernstige complicaties en heeft een hoge kans op sterfte. Vanaf 2010 werden we in onze regio steeds meer geconfronteerd met patiënten met geïnfecteerde vaten. Om deze patiënten beter te herkennen en te behandelen verrichten we een review van publicaties over patiënten met Qkoorts vaatwandinfecties. In hoofdstuk 7 worden de resultaten van deze review beschreven Uit de analyse van 58 patiënten kwam naar voren dat pijn en koorts vaak voorkwamen bij presentatie. Daarnaast was vaak bij eerste presentatie al sprake van een ernstige complicatie, zoals fistelvorming, abcessen en trombo-embolische complicaties. Het merendeel $(80 \%)$ werd naast antibiotisch ook chirurgisch behandeld. Het sterfterisico was $24 \%$, waarbij de sterfte in de conservatief behandelde groep significant hoger was dan in de groep patiënten die een resectie ondergingen van de geinfecteerde vaatwand. Op basis van deze analyse is voorzichtig te concluderen dat chirurgische interventie een integraal onderdeel van de behandeling van vasculaire wandinfecties bij chronische Q-koorts hoort te zijn.

In hoofdstuk 8 wordt opnieuw het belang van een chirurgische ingreep geïllustreerd door de beschrijving van een patiënt met een geïnfecteerde aorta ascendens prothese. Een langdurige behandeling met antibiotica was niet succesvol: onder antibiotica was geen afname van de infectie, uiteindelijk volgde stabilisatie na het vervangen van de prothese.

Vanaf 2010 werden we geconfronteerd met tientallen chronische Q-koorts patiënten, vijf patiënten stierven kort na opname als gevolg van ernstige complicaties. Opvallend was dat de meeste patiënten niet met acute Qkoorts waren gediagnosticeerd en zich geen ziektebeeld passend bij acute Q-koorts konden herinneren. Het gevoel dat we slechts een topje van de ijsberg zagen, was de aanleiding om patiënten met risico op chronische Qkoorts te gaan screenen. In hoofdstuk 9 tonen we aan dat een screeningsprogramma voor chronische Q-koorts na een acute $\mathrm{Q}$-koorts epidemie een goede benadering lijkt te zijn in het detecteren van chronische Q-koorts patienten in een vroeg stadium. Patiënten die in aanmerking kwamen voor screening waren patiënten met een aneursyma van de aorta, en met aortaen/of hartklepprothesen. In de groep van symptomatische chronische Qkoorts patiënten die werden gevonden in onze regio bleken deze drie factoren bijna altijd aanwezig te zijn. In een cohort van 763 patiënten werden 10 
nieuwe chronische Q-koorts patiënten gevonden, waarvan twee bewezen en acht waarschijnlijke chronische Q-koorts. Prothesen bleek een belangrijke risicofactor te zijn. Alle patiënten hadden vasculaire prothesen van de aorta of van de hartklep. Slechts bij één patiënt werden klinische symptomen, zoals koorts, waargenomen. Bij zeven patiënten werd behandeling met antibiotica gestart. Hoewel een kosten- batenanalyse niet werd uitgevoerd in onze studie is het denkbaar dat een screeningsprogramma, zoals geïnitieerd in onze ziekenhuizen kosteneffectief is. De kosten van serologische tests per patiënt zijn verwaarloosbaar vergeleken met de kosten van de behandeling van symptomatische chronische Q-koorts patiënten.

\section{Conclusie}

In dit proefschrift is de waarde van de verschillende diagnostische tests voor acute en chronische Q-koorts onderzocht. Met behulp van serologie en PCR is de diagnose acute Q-koorts adequaat te stellen, vooral wanneer beide testen worden gecombineerd in een algoritme. Door de langdurige halfwaardetijd van antistoffen, zijn aanpassingen in die algoritmen en interpretatie van de testresultaten vereist in postepidemische gebieden. In tegenstelling tot acute Q-koorts is de diagnostiek van de chronische infectie niet altijd gemakkelijk. De huidige microbiologische testen zijn niet altijd toereikend: de gevoeligheid van de PCR-test is laag en de uitslag van een serologische test is slechts een hulpstuk in de diagnostiek en biedt weinig steun als marker voor antibiotische behandeling. De diagnose van chronische Q-koorts is derhalve moeilijk en niet alleen op basis van microbiologische testen te stellen, maar is een gecombineerde diagnose, inclusief klinische aspecten en resultaten van beeldvorming. Nieuwe testen, gebaseerd op verschillende principes, zijn dringend nodig, ook voor de follow-up van chronische Qkoorts patiënten.

Naast diagnostische analyses werden de klinische aspecten van chronische Q-koorts patiënten bestudeerd in dit proefschrift. We lieten zien dat een chirurgische ingreep een vast onderdeel van de behandeling van vasculaire geïnfecteerde patiënten zou moeten zijn. Het bestaan van asymptomatische chronische Q-koorts werd gedemonstreerd tijdens een Q-koorts screeningprogramma.

Een aantal van de studies in dit proefschrift werden ingebed in de nationale Q-koorts consensusgroep. Deze multidisciplinaire aanpak zou model kunnen staan voor toekomstige uitbraken met opkomende micro-organismen: ten 
eerste, het installeren van een team van experts in het veld; ten tweede, het verzamelen en delen van de beschikbare kennis; en ten derde, bepaal samen de belangrijkste 'witte vlekken' waarvoor het onderzoek moet worden gestart. 

Dankwoord 
Dit hoofdstuk is bedoeld om alle mensen te bedanken die mij hebben geholpen bij mijn promotie. Ook alle mensen die mij daarbuiten gesteund hebben en de kracht en aandacht hebben gegeven om het 'project' af te ronden wil ik heel hartelijk bedanken. Een behoorlijk aantal mensen wil ik graag met naam noemen.

Allereerst mijn beide promotoren: prof. dr. J.A.W. Teijink en prof. dr. M.P.G. Koopmans. Een vaatchirurg en een dierenarts/viroloog, twee specialisten die elkaar anders niet vaak ontmoeten. Tijdens de Q-koorts epidemie in Nederland hebben we geleerd gezamenlijk, klinisch specialisten en laboratorium specialisten, op te trekken. Daarom ben ik zeer verheugd dat jullie beiden mijn promotor wilden zijn.

Beste Joep, de ontmoeting met jou in de lift van het Catharina Ziekenhuis "dr. Teijink I presume?" was de start van een mooie samenwerking, waarbij jij direct de ernst van de situatie begreep en de zorg voor de chronische gevolgen voor patiënten met vaat- en hartklepproblemen met mij deelde. Dankzij jou ging de organisatie voor het screenen op chronische Q-koorts lopen.

Beste Marion, jij hebt me geïntroduceerd in de wereld van 'public health'. Je hebt mij daarbij de essentie van een goede samenwerking tussen laboratoria, epidemiologen, overheden en zorgverleners bijgebracht. Dank voor je kritische en creatieve bijdrage aan de onderzoeken. Het feest met de EUPHEM/EPIET graduates in Edinborough was geweldig!

Dank voor jullie vertrouwen in een goede afloop van mijn promotie.

Mijn beide copromotoren, dr. H.T. Tjhie en dr. H.A. Bijlmer.

Beste Jeroen, een rots in de branding was je/ben je. Als ik met handen in het haar naar het commentaar van reviewers keek was jij degene die allereerst duidelijkheid en overzicht creëerde. En dat ook nog in een korte tijd. Het was nooit nodig je een reminder te sturen. Dank ook voor je kritische inbreng en je uitgebreide kennis van zaken.

Beste Henk, als voorzitter van de Q-koorts consensus groep, was jij degene die de mensen van de verschillende ziekenhuizen en afdelingen bij elkaar bracht. Je hebt ervoor gezorgd dat de aftastende houding in het begin omsloeg in samenwerking. De output en de internationale aandacht voor ons consensusmodel zijn daardoor mede jouw verdiensten. 
Graag wil ik de beoordelingscommissie bestaande uit prof. dr. C.J.P.A. Hoebe, prof. dr. P.H.H. Savelkoul, dr. C.M. Verduin en dr. A. Oude Lashof bedanken voor het beoordelen van mijn manuscript.

Dank aan de leden van de consensus groep. Dank ook aan de andere Qkoorts onderzoekers. Met een aantal van jullie zijn mooie publicaties verschenen. Een paar mensen wil ik in het bijzonder bedanken. Peter Schneeberger, mijn eerste voorzichtige stapjes in het Q-koorts onderzoek leidde onder jouw aansturing tot een publicatie over de waarde van de serologie tijdens en na de uitbraak. Dank ook aan andere collega's uit Den Bosch, zoals Nicole Renders, Peter Wever en Lieke Wielders voor de prettige samenwerking en voor het delen van jullie kennis. Linda Kampschreur, de door jouw gemaakte chronische Q-database is van grote waarde. Ik kijk met veel trots terug op de manier waarop wij samen de 700+ artikelen voor de review hebben doorgespit op zoek naar bruikbare analyses. De Nijmeegse Club, met onder andere Marrigje Nabuurs-Franssen en Teske Schoffelen, altijd bereid tot overleg en samenwerken. Teske, jouw gedegen onderzoek naar hét interleukineprofiel van Q-koorts patiënten leidt zeker tot een mooi proefschrift! Dank aan de collega's uit mijn ziekenhuis, Marjolijn Pronk, Yvonne Soethoudt, Pepijn van de Voort, Marc van Sambeek, Tryfon Vainas, Joost ter Woorst, Bart van Straten, Volkher Scharnhorst en uit de andere ziekenhuizen voor het 'aanleveren' van patiëntgegevens, het verzamelen van materialen en het mee publiceren. Aan de manier waarop wij in Nederland de kennis over chronische Q-koorts poolen, bespreken en publiceren kan menig Fransman een puntje zuigen. Giovanna Jaramillo-Gutierrez, it was so nice meeting you and to learn about your global way of life. I really hope we keep in touch. Dank aan de collega's van het RIVM, waaronder Wim van der Hoek en Tineke Herremans en Ronald ter Schegget van de GGD BrabantZuidoost. Q-koorts is een multidisciplinaire aandoening, ik heb via jullie veel geleerd van de 'public health' kant van het verhaal. Rutger Stokmans, dank voor het aanleveren van de patiëntenbestanden en je bijdrage in ons artikel over het screenen van patiënten.

Zonder de collega's van de PAMM was er geen boekje. Ik heb ongelooflijk veel steun en helpende handen gekregen van diverse afdelingen van de PAMM. René van Aken, geweldig bedankt voor de coördinatie op de afdeling serologie. Klinische sera, onderzoeksera, tussendoorsera, ongevraagde 
sera... Je bleef altijd kalm en behield het overzicht. Babs Korting, Angela Kruijsdijk, Esther Vissers, Sigrid Merkx, Leonie Bartels, Ronnie van Gessel, Frank Trommelen, Adrie Staals en de anderen van de serologie, jullie hebben duizenden sera gepipetteerd. Zeer veel dank voor jullie inzet. Het medisch secretariaat, Ans Bressers en Jorien van Dungen, dank voor jullie (mentale) support en ondersteuning van een steeds ingewikkelder logistiek bedrijf met uitvliegende artsen-microbioloog. Jorien, veel dank voor de coördinatie van de screening. Ik weet $100 \%$ zeker dat jouw inbreng er voor heeft gezorgd dat er geen brief op de verkeerde deurmat is gevallen. Dank ook aan de collega's van de 'ondersteunende diensten' zoals Jacob van den Berg en Rukiye Arici. Nicole Verhofstad, dank voor de onmisbare hulp in het afronden van het manuscript en de administratie eromheen.

Speciale dank gaat uit naar Joris Korbeeck. Joris, heel veel dank voor al je ICT en statistische ondersteuning en je engelengeduld. Je hebt je onmisbaar gemaakt binnen de PAMM door je groot analytisch vermogen en je charme.

De collega artsen-microbioloog van de PAMM, Arjan Jansz, Niek Arents, Laura van Dommelen, Jeroen Tjhie, Sandra Erkens, Patrick Sturm, Lieven van de Velden en Jeroen van de Bovenkamp, we werken in een dynamische omgeving. De kracht van ons team zit in onze saamhorigheid: één voor allen, allen voor één. De werksfeer op de PAMM is professioneel en plezierig. Ook met de andere collega's 'langs mij' op de gang, Theo Liebregts en René Roymans en alle andere werknemers van de PAMM is het prettig samenwerken. Ik dank het secretariaat van de PA voor alle versnaperingen, ik hoop dat mijn bijdrage aan de snoeppot voldoende en gevarieerd genoeg is. Ook dank ik de pathologen voor hun interesse en gezelligheid.

Naast de PAMM in Veldhoven ook werkzaam zijn in het Catharina Ziekenhuis in Eindhoven vergt de nodige coördinatie. Maar mijn werkzaamheden in het Catharina Ziekenhuis zou ik voor geen goud willen missen. Ik voel mij er thuis. Ilse Laros, Marieke Ernest, Ada Janson, Monique MoerenhoutVerhoeven, Margot van Hooijdonk, Hanneke Beelen van de afdeling ziekenhuishygiëne en infectiepreventie en Carien Miedema, kinderarts, maar vooral infectiecommissie voorzitter. Het zijn drukke tijden, never a dull moment, jullie doen het geweldig en ik dank jullie voor de samenwerking, die ondanks de hectiek en drukte goed blijft. 
Mireille Wulf en Michiel Blans, geweldig dat jullie mijn paranimfen willen zijn. Mireille, ik heb jouw promotie van dichtbij mee mogen maken. Er was geen speld tussen te krijgen, voor mij een goed voorbeeld. Je hebt je hart (en je honden) gevolgd. Dat je ons op de PAMM daarvoor moest verlaten heb ik je inmiddels vergeven. Michiel, grotere broer en collega, heerlijk dat je straks naast me staat, dat geeft een veilig gevoel.

Dank aan mijn vrienden, Nuenense buddies, Deuzjevoo vriendinnen, bergsteigers. Dank voor jullie support, belangstelling en gezelligheid.

Loes van der Meulen, onmisbare schakel in ons gezin, dank voor al je hulp van de afgelopen vijf jaar! Loes, we gaan dat lustrum vieren!

Lieve Bart, Michiel en Nienke, "lieve broeders en zusters". Ik ben trots op onze familieband, we delen hetzelfde verdriet en dezelfde humor. Er zijn, denk ik maar weinig families die een week met de gezinnen samen op een berg in Frankrijk kunnen vertoeven. Lieve schoonfamilie Fatma, Aleid, Yet, Francisca, Jacomijn, Joost, Eppo, Merijn, neefjes en nichtjes en lieve schoonouders Mariëtta en Harry, dank voor jullie interesse en gezelligheid. Dank dat jullie er zijn. Voor onze families geldt: de toename van de kwantiteit doet geen afbreuk aan de kwaliteit: wij blijven elkaar altijd interesseren. Lieve Harry, dank voor je creatieve hulp bij de laatste losse eindjes van 'het boekje'.

Lieve Johannes, mijn lief en mijn maatje, al 21 jaar! Je hebt mij de ruimte gegeven om dit project af te ronden en je hebt mij van veel taken ontlast. Masha danki mi dushi. We hebben een heerlijk en vol leven, samen met onze schatjes Mees, Piet Hein en Floortje. Jullie vier zijn het allerbelangrijkst. 

Curriculum Vitae 

Marjolijn Wegdam-Blans werd op 23 februari 1970 te Leiderdorp geboren. De middelbare school (VWO) werd doorlopen in Leiden. Na 1 jaar Scheikunde te hebben gestudeerd, werd in 1989 begonnen met de studie Geneeskunde aan de Rijksuniversiteit te Groningen. Co-schappen werden doorlopen in het St. Elisabeth ziekenhuis te Curaçao. Na het behalen van het artsenexamen was zij 1 jaar werkzaam als arts-assistent niet in opleiding in het Willem Alexander Ziekenhuis te Den Bosch. In 1998 was zij als expeditie-arts betrokken bij een succesvolle beklimming van de Cho Oyu $(8201 \mathrm{~m}$, Tibet). Daarna werd begonnen met de opleiding Medische Microbiologie te Utrecht (opleiders: prof. dr. J. Verhoef (UMCU) en dr. R. Diepersloot (Diakonessenhuis)). $\mathrm{Na}$ afronding van de opleiding (in 2004) was zij eerst werkzaam als arts-microbioloog in Apeldoorn en later (in 2008) in Enschede. Per februari 2009 is zij werkzaam als arts-microbioloog bij stichting PAMM te Veldhoven en tevens in het Catharina Ziekenhuis te Eindhoven. Op het moment van in dienst treding was de Q-koorts epidemie op zijn hoogtepunt. Medio 2010 werd begonnen met aanvang van de onderzoeken beschreven in dit proefschrift. Marjolijn is getrouwd met Johannes en samen hebben ze drie kinderen, Mees, Piet Hein en Floortje. 



\section{List of publications}


Elsa J. van den Berg, Lieke Wielders, Peter M. Schneeberger, Marjolijn C. Wegdam-Blans and Wim van der Hoek. Spatial analysis of positive and negative $Q$ fever laboratory results for identifying 3 high- and low-risk areas of infection in the Netherlands. Infection Ecology and Epidemiology 2013, 3: 20432.

Schoffelen T, Sprong T, Bleeker-Rovers CP, Wegdam-Blans MC, Ammerdorffer A, Pronk MJ, Soethoudt YE, van Kasteren ME, Herremans T, Bijlmer HA, Netea MG, van der Meer JW, Joosten LA, van Deuren M. A Combination of IFN-y and IL-2 Production by Coxiella burnetii Stimulated Circulating Cells Discriminates Between Chronic Q Fever and Past Q Fever. Clin Microbiol Infect. 2013 Oct 9. doi:10.1111/1469-0691.12423.

G Jaramillo-Gutierrez, MC Wegdam-Blans, R ter Schegget, JM Korbeeck, R van Aken, HA Bijlmer, JH Tjhie, MP Koopmans. A dynamic case definition is warranted for adequate notification in an extended epidemic setting: the Dutch Q fever outbreak 2007-2009 as exemplar. Eurosurveillance, Volume 18, Issue 41, 10 October 2013.

Wegdam-Blans MC, Stokmans RA, Tjhie JH, Korbeeck JM, Koopmans MP, Evers SM, van der Voort PH, Teijink JA. Targeted screening as a tool for the early detection of chronic $Q$ fever patients after a large outbreak. Eur J Clin Microbiol Infect Dis. 2013 Mar;32(3):353-9.

Herremans T, Hogema BM, Nabuurs M, Peeters M, Wegdam-Blans $M$, Schneeberger P, Nijhuis C, Notermans DW, Galama J, Horrevorts A, van Loo IH, Vlaminckx B, Zaaijer HL, Koopmans MP, Berkhout H, Socolovschi C, Raoult D, Stenos J, Nicholson W, Bijlmer H. Comparison of the performance of IFA, CFA, and ELISA assays for the serodiagnosis of acute $Q$ fever by quality assessment. Diagn Microbiol Infect Dis. 2013 Jan;75(1):16-21.

Morroy G, Wielders CC, Kruisbergen MJ, van der Hoek W, Marcelis JH, Wegdam-Blans MC, Wijkmans CJ, Schneeberger PM. Large regional differences in serological follow-up of $Q$ fever patients in the Netherlands. PLoS One. 2013;8(4):e60707. 
van Vuuren TM, Wegdam JA, Wegdam-Blans MC. [Intestinal perforation caused by Salmonella typhimurium]. Ned Tijdschr Geneeskd. 2013;157(24):A6161. Dutch.

van der Hoek W, Wielders CC, Schimmer B, Wegdam-Blans MC, Meekelenkamp J, Zaaijer HL, Schneeberger PM. Detection of phase I IgG antibodies to Coxiella burnetii with EIA as a screening test for blood donations. Eur $\mathrm{J}$ Clin Microbiol Infect Dis. 2012 Nov;31(11):3207-9.

Kampschreur LM, Wever PC, Wegdam-Blans MC, Delsing CE, BleekerRovers CP, Sprong T, van Kasteren ME, Coutinho RA, Schneeberger PM, Notermans DW, Bijlmer HA, Koopmans MP, Nabuurs-Franssen MH, Oosterheert JJ. Defining chronic Q fever: a matter of debate. J Infect. 2012 Oct;65(4):362-3.

Algin A, Wegdam-Blans MC, Verduin $\mathrm{K}$, Janssen $\mathrm{H}$, van Dantzig JM. Tropheryma whipplei aortic valve endocarditis, cured without surgical treatment. BMC Res Notes. 2012 Oct 30;5:600.

Wegdam-Blans MC, Wielders CC, Meekelenkamp J, Korbeeck JM, Herremans T, Tjhie HT, Bijlmer HA, Koopmans MP, Schneeberger PM. Evaluation of commonly used serological tests for detection of Coxiella burnetii antibodies in well-defined acute and follow-up sera. Clin Vaccine Immunol. 2012 Jul;19(7):1110-5.

Wegdam-Blans MC, ter Woorst JF, Klompenhouwer EG, Teijink JA. David procedure during a reoperation for ongoing chronic $Q$ fever infection of an ascending aortic prosthesis. Eur J Cardiothorac Surg. 2012 Jul;42(1):e19-20.

Kampschreur LM, Oosterheert JJ, Koop AM, Wegdam-Blans MC, Delsing CE, Bleeker-Rovers CP, De Jager-Leclercq MG, Groot CA, Sprong T, Nabuurs-Franssen MH, Renders NH, van Kasteren ME, Soethoudt Y, Blank SN, Pronk MJ, Groenwold RH, Hoepelman AI, Wever PC. Microbiological challenges in the diagnosis of chronic Q fever. Clin Vaccine Immunol. 2012 May;19(5):787-90. 
Wegdam-Blans MC, Kampschreur LM, Delsing CE, Bleeker-Rovers CP, Sprong $T$, van Kasteren ME, Notermans DW, Renders NH, Bijlmer HA, Lestrade PJ, Koopmans MP, Nabuurs-Franssen MH, Oosterheert JJ; Dutch $Q$ fever Consensus Group. Chronic $Q$ fever: review of the literature and a proposal of new diagnostic criteria. J Infect. 2012 Mar;64(3):247-59.

van der Hoek W, Schneeberger PM, Oomen T, Wegdam-Blans MC, Dijkstra F, Notermans DW, Bijlmer HA, Groeneveld K, Wijkmans CJ, Rietveld A, Kampschreur LM, van Duynhoven Y. Shifting priorities in the aftermath of a Q fever epidemic in 2007 to 2009 in The Netherlands: from acute to chronic infection. Euro Surveill. 2012 Jan 19;17(3):20059.

Wegdam-Blans MC, Vainas T, van Sambeek MR, Cuypers PW, Tjhie HT, van Straten AH, Teijink JA. Vascular complications of $Q$-fever infections. Eur J Vasc Endovasc Surg. 2011 Sep;42(3):384-92.

Kampschreur LM, Wegdam-Blans MC, Thijsen SF, Groot CA, Schneeberger PM, Hollander AA, Schijen JH, Arents NL, Oosterheert JJ, Wever PC. Acute $Q$ fever related in-hospital mortality in the Netherlands. Neth J Med. 2010 Dec;68(12):408-13.

Schimmer B, Ter Schegget R, Wegdam M, Züchner L, de Bruin A, Schneeberger PM, Veenstra T, Vellema P, van der Hoek W. The use of a geographic information system to identify a dairy goat farm as the most likely source of an urban Q-fever outbreak. BMC Infect Dis. 2010 Mar 16;10:69.

Wegdam-Blans MC, Nabuurs-Franssen MN, Horrevorts AM, Peeters MF, Schneeberger PM, Bijlmer HA. [Laboratory diagnosis of acute $Q$ fever]. Ned Tijdschr Geneeskd. 2010;154:A2388. Review. Dutch.

Blans MC, Visseren FL, Banga JD, Hoekstra JB, van der Graaf Y, Diepersloot RJ, Bouter KP. Infection induced inflammation is associated with erectile dysfunction in men with diabetes. Eur J Clin Invest. 2006 Jul;36(7):497502. 
Salimans MM, Hol C, Blans MC, van der Zanden AG. Use of urine samples as controls for treatment of a Chlamydia trachomatis infection. J Med Microbiol. 2006 Feb;55(Pt 2):245-6.

Blans MC, Ridwan BU, Verweij JJ, Rozenberg-Arska M, Verhoef J. Cyclosporiasis outbreak, Indonesia. Emerg Infect Dis. 2005 Sep;11(9):1453-5.

Blans MC, Troelstra A. Glycopeptide resistance in Staphylococcus haemolyticus during treatment with teicoplanin. Infect Control Hosp Epidemiol. 2001 May;22(5):263-4.

Ligtenberg PC, Blans MC, Hoekstra JB, van der Tweel I, Erkelens DW. No effect of long-term physical activity on the glycemic control in type 1 diabetes patients: a cross-sectional study. Neth J Med. 1999 Aug;55(2):59-63.

Blans MC, Conemans JM, van der Veer JK, Bouter KP. [Factitious insulinoma]. Ned Tijdschr Geneeskd. 1998 Sep 26;142(39):2154-6. Dutch. 
